\title{
Las Redes de Petri Interpretadas en el Diagnóstico de Fallos de Sistemas Híbridos. Aplicación a un Helicóptero no Tripulado
}

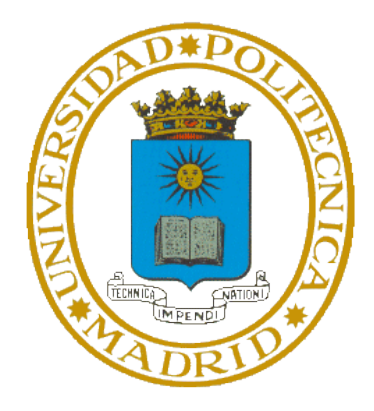

\section{Miguel Angel Trigos Martínez}

Departamento de Automática, Ingeniería Electrónica e Informática Industrial Universidad Politécnica de Madrid

Tesis presentada para obtener el grado de:

Doctor Ingeniero Industrial en Automática y Robótica

Enero 2016 
Titulo:

Las Redes de Petri Interpretadas en el Diagnóstico de Fallos de Sistemas Híbridos. Aplicación a un Helicóptero no

Tripulado

Autor:

Miguel Angel Trigos Martínez, Ing.

Director

Prof. Antonio Barrientos Cruz, Ph.D

Prof. Jaime Del Cerro Giner, Ph.D

Grupo de Robótica y Cibernética

Tribunal nombrado por Mgnfco. y Excmo. Sr. Rector de la Universidad

Politécnica de Madrid, el día de de 2016.

\section{Tribunal}

Presidente: Dr. D. AGUSTÍN JIMÉNEZ AVELLO

Vocal: $\quad$ Dr. D. JOSÉ MARÍA CAÑAS PLAZA

Vocal: $\quad$ Dr. D. RAMÓN IGNACIO BARBER CASTAÑO

Vocal: $\quad$ Dr. D. JOSÉ JAVIER RAINER GRANADOS

Secretario: Dr. D. ERNESTO GAMBAO GALÁN

Suplente: Dr. D. JOÃO RICARDO PEREIRA VALENTE

Suplente: Dr. D. DIEGO GACHET PAEZ

Realizado el acto de lectura y defensa de la tesis el día de de 2016.

Calificación de la Tesis:

El Presidente:

Los Vocales:

El Secretario: 


\section{Abstract}

Safety and reliability of industrial processes are the main concern of the engineers in charge of industrial plants. Thus, from an economic point of view, the main goal is to reduce the maintenance downtime cost and the losses caused by failures. Moreover, the safety of the operators, which affects to social and economic aspects, is the most relevant factor to consider in any system.

Due to this, fault diagnosis has become a relevant focus of interest for worldwide researchers and engineers in the industry.

The main works focused on failure detection are based on models of the processes. There are different techniques for modelling industrial processes such as state machines, decision trees and Petri Nets (PN). Thus, this Thesis is focused on modelling processes by using Interpreted Petri Nets.

Petri Nets is a tool used in the graphic and mathematical modelling with ability to describe information of the systems in a concurrent, parallel, asynchronous, distributed and not deterministic or stochastic manner. PNs are also useful graphical visual communication tools as flow chart or block diagram. Additionally, the marks of the PN simulate the dynamics and concurrence of the systems. Finally, they are able to define specific state equations, algebraic equations and other models that represent the common behaviour of systems. Among the different types of PN (Interpreted, Coloured, etc.), this research work deals with the interpreted Petri Nets mainly due to features such as synchronization capabilities, timed places, apart from their capability for processing data.

This Research begins with the process for designing and building the model and diagnoser to detect permanent faults, subsequently, the temporal dynamic was added for detecting intermittent faults. Two industrial processes, namely 
HVAC (Heating, Ventilation and Air Condition) and Liquids Packaging Process were used as testbed for implementing the Fault Diagnosis (FD) tool created. Finally, its diagnostic capability was enhanced in order to detect faults in hybrid systems.

Finally, a small unmanned helicopter was chosen as example of system where safety is a challenge and fault detection techniques developed in this Thesis turn out to be a valuable tool since UAVs accidents involve high economic cost and are the main reason for setting restrictions to fly over populated areas. Thus, this work introduces a systematic process for building a Fault Diagnoser of the mentioned system based on Petri Nets. This novel tool is able to detect both intermittent and permanent faults. The work carried out is discussed from theoretical and practical point of view. The procedure begins with a division of the system into subsystems for further integration into a global PN diagnoser that is able to monitor the whole system and show critical variables to the operator in order to determine the UAV health, preventing accidents in this manner.

A Data Acquisition System (DAQ) has been also designed for collecting data during the flights and feed PN Diagnoser. Real flights carried out under nominal and failure conditions have been required to perform the diagnoser setup and verify its performance. It is worth noting that a high risk was assumed in the generation of faults during the flights, nevertheless this allowed collecting basic data so as to develop fault diagnosis, isolations techniques, maintenance protocols, behaviour models, etc.

Finally, a summary of the validation results obtained during real flight tests is also included. An extensive use of this tool will improve preventive maintenance protocols for UAVs (especially helicopters) and allow establishing recommendations in regulations. The use of the diagnoser by using Petri Nets is considered as novel approach. 


\section{Resumen}

La seguridad y fiabilidad de los procesos industriales son la principal preocupación de los ingenieros encargados de las plantas industriales. Por lo tanto, desde un punto de vista económico, el objetivo principal es reducir el costo del mantenimiento, el tiempo de inactividad y las pérdidas causadas por los fallos. Por otra parte, la seguridad de los operadores, que afecta a los aspectos sociales y económicos, es el factor más relevante a considerar en cualquier sistema

Debido a esto, el diagnóstico de fallos se ha convertido en un foco importante de interés para los investigadores de todo el mundo e ingenieros en la industria.

Los principales trabajos enfocados en detección de fallos se basan en modelos de los procesos. Existen diferentes técnicas para el modelado de procesos industriales tales como máquinas de estado, árboles de decisión y Redes de Petri (RdP). Por lo tanto, esta tesis se centra en el modelado de procesos utilizando redes de petri interpretadas.

Redes de Petri es una herramienta usada en el modelado gráfico y matemático con la habilidad para describir información de los sistemas de una manera concurrente, paralela, asíncrona, distribuida y no determinística o estocástica. RdP son también una herramienta de comunicación visual gráfica útil como lo son las cartas de flujo o diagramas de bloques. Adicionalmente, las marcas de las RdP simulan la dinámica y concurrencia de los sistemas. Finalmente, ellas tienen la capacidad de definir ecuaciones de estado específicas, ecuaciones algebraicas y otros modelos que representan el comportamiento común de los sistemas. Entre los diferentes tipos de redes de petri (Interpretadas, Coloreadas, etc.), este trabajo de investigación trata con redes de petri interpretadas principalmente debido a características tales como sincronización, lugares temporizados, aparte de su capacidad para procesamiento de datos.

Esta investigación comienza con el proceso para diseñar y construir el modelo y diagnosticador para detectar fallos definitivos, posteriormente, la dinámica 
temporal fue adicionada para detectar fallos intermitentes. Dos procesos industriales, concretamente un HVAC (Calefacción, Ventilación y Aire Acondicionado) y un Proceso de Envasado de Líquidos fueron usados como banco de pruebas para implementar la herramienta de diagnóstico de fallos (FD) creada. Finalmente, su capacidad de diagnóstico fue ampliada en orden a detectar fallos en sistemas híbridos.

Finalmente, un pequeño helicóptero no tripulado fue elegido como ejemplo de sistema donde la seguridad es un desafío, y las técnicas de detección de fallos desarrolladas en esta tesis llevan a ser una herramienta valorada, desde que los accidentes de las aeronaves no tripuladas (UAVs) envuelven un alto costo económico y son la principal razón para introducir restricciones de volar sobre áreas pobladas. Así, este trabajo introduce un proceso sistemático para construir un Diagnosticador de Fallos del sistema mencionado basado en RdP. Esta novedosa herramienta es capaz de detectar fallos definitivos e intermitentes. El trabajo realizado es discutido desde un punto de vista teórico y práctico. El procedimiento comienza con la división del sistema en subsistemas para seguido integrar en una RdP diagnosticadora global que es capaz de monitorear el sistema completo y mostrar las variables críticas al operador en orden a determinar la salud del UAV, para de esta manera prevenir accidentes.

Un Sistema de Adquisición de Datos (DAQ) ha sido también diseñado para recoger datos durante los vuelos y alimentar la RdP diagnosticadora. Vuelos reales realizados bajo condiciones normales y de fallo han sido requeridos para llevar a cabo la configuración del diagnosticador y verificar su comportamiento. Vale la pena señalar que un alto riesgo fue asumido en la generación de fallos durante los vuelos, a pesar de eso esto permitió recoger datos básicos para desarrollar el diagnóstico de fallos, técnicas de aislamiento, protocolos de mantenimiento, modelos de comportamiento, etc.

Finalmente, un resumen de la validación de resultados obtenidos durante las pruebas de vuelo es también incluido. Un extensivo uso de esta herramienta mejorará los protocolos de mantenimiento para UAVs (especialmente helicópteros) y permite establecer recomendaciones en regulaciones. El uso del diagnosticador usando redes de petri es considerado un novedoso enfoque. 


\section{Agradecimientos}

A los profesores Antonio Barrientos Cruz y Jaime Del Cerro Giner, directores de la tesis, por su colaboración, paciencia y profesionalidad. A la Universidad Santo Tomás de Bucaramanga (Colombia) quien ha patrocinado el inicio de mis estudios y especialmente al Padre Guillermo León Villa Hincapié O.P, gran persona y amigo de la academia. 


\section{Dedicatoria}

A mi familia y especialmente a mis padres, Miguel Angel y Beatriz, por darme la vida y enseñarme todo lo que soy A mis hijos Miguel Angel y Gabriela, razón para vivir eternamente A mi esposa Jisseth, por su paciencia y comprensión

Miguel Angel 


\section{Índice general}

Índice General $\quad$ X

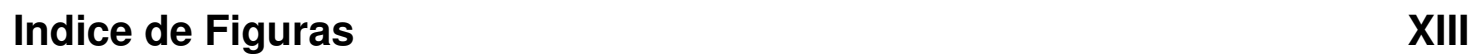

$\begin{array}{ll}\text { Lista de Tablas } & \text { XVI }\end{array}$

$\begin{array}{ll}\text { Nomenclatura } & \text { XVIII }\end{array}$

1. Introducción 1

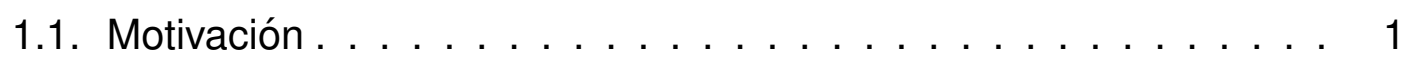

1.2. Objetivos y Alcance . . . . . . . . . . . . 3

1.3. Principales Contribuciones de la Tesis . . . . . . . . . . 5

1.4. Organización de la Tesis . . . . . . . . . . . . . . . 5

2. Trabajos Previos en Diagnóstico de Fallos 7

2.1. Conceptos en Diagnóstico de Fallos . . . . . . . . . . . 8

2.1.1. Clases de Fallos de un Sistema . . . . . . . . . . . 8

2.1.2. Características Deseables de un Sistema de Diagnóstico de Fallos . . . . . . . . . . . . . . . . 10

2.2. Clasificación de los Algoritmos de Diagnóstico según el Conocimiento a Priori . . . . . . . . . . . . . . . . . . . . 14

2.2.1. Algoritmos Basados en Modelos Cuantitativos . . . . . . 16

2.2.2. Algoritmos Basados en Modelos Cualitativos . . . . . . . 22

2.2.3. Métodos Basados en Historia del Proceso . . . . . . . . . . 30

2.3. Trabajos Previos en Diagnóstico de Fallos de Sistemas de Eventos Discretos . . . . . . . . . . . . . . . . 36

2.4. Trabajos Previos en Diagnóstico de fallos de Sistemas Híbridos . . 39

2.5. Trabajos Previos en Diagnóstico de Fallos de UAV's . . . . . . . 42 
2.5.1. Métodos Basados en Modelo Analítico. . . . . . . . . . . . 43

2.5.2. Métodos basados en Procesamiento de Señales. . . . . . . 45

2.5.3. Métodos Basado en Conocimiento. . . . . . . . . . . . . . . 46

3. Fundamentos de Redes de Petri 47

3.1. Conceptos Básicos . . . . . . . . . . . . . . . . . . . . . . . . 47

3.2. Propiedades Representativas de una RdP . . . . . . . . . . . . . 49

3.3. Álgebra Lineal. . . . . . . . . . . . . . . . . . . . . . . 50

3.4. Redes de Petri No-Autónomas . . . . . . . . . . . . . . 51

3.5. Redes de Petri Autónomas . . . . . . . . . . . . . . . . . . 54

4. Construcción del Modelo y Diagnosticador usando RdP para FD de SED's

4.1. Características Importantes de un FDI en SED's . . . . . . . . 60

4.2. Construcción del Modelo . . . . . . . . . . . . . . . . 61

4.3. Operación de Integración y Refinamiento del Modelo. . . . . . . 63

4.4. El Diagnosticador y la Diagnosticabilidad . . . . . . . . . 66

4.4.1. Construcción del Diagnosticador . . . . . . . . . . . 67

4.4.2. Diagnosticabilidad . . . . . . . . . . . 71

4.5. Ejemplo de Aplicación . . . . . . . . . . . . . . . . . 72

4.5.1. Ejemplo de Sistema HVAC (Heating, Ventilation and Air Condition) . . . . . . . . . . . . . . . . 72

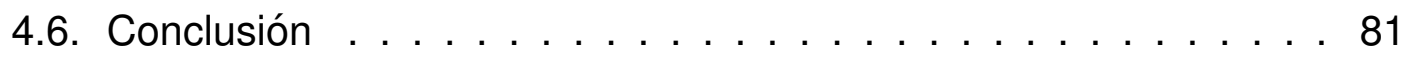

5. Adición de la Dinámica Temporal al Diagnosticador 83

5.1. Modelo y Diagnosticador para Fl's . . . . . . . . . . . . 85

5.2. Diagnóstico Temporal de Fl's . . . . . . . . . . . . . . . 87

5.3. Ejemplo Proceso de Envasado . . . . . . . . . . . . . . 92

6. Proceso de Construcción del Diagnosticador usado en Sistemas Híbridos $\quad 101$

6.1. Introducción . . . . . . . . . . . . . . . . . . . . 101

6.2. Herramienta de Diagnóstico . . . . . . . . . . . . . . . . . 104

6.2.1. Proceso de Construcción del Modelo y Diagnosticador . . . 105

6.2.2. Diagnosticabilidad . . . . . . . . . . . . . . 115

6.3. Ejemplo de Aplicación, Sistema de Envasado de Líquidos . . . . 116 
ÍNDICE GENERAL

6.3.1. Modelamiento continuo del Sistema de Envasado . . . . 117

6.3.2. Aplicación del Proceso de Construcción del Diagnosticador 120

7. Implementación y Pruebas de Campo del Diagnosticador en el HC no Tripulado. 127

7.0.1. Introducción . . . . . . . . . . . . . . . . . . . 127

7.0.2. El Helicóptero no Tripulado y el Sistema de Adquisición de Datos . . . . . . . . . . . . . . . . 130

7.1. Aplicación de la Herramienta de FD al Helicóptero . . . . . . . 142

7.1.1. Análisis de Resultados. . . . . . . . . . . . . . . . . . . . 162

$\begin{array}{ll}\text { 8. Conclusión } & 181\end{array}$

$\begin{array}{ll}\text { Referencias } & 185\end{array}$

$\begin{array}{ll}\text { Apéndice } & 197\end{array}$

A. Unmanned Aerial Systems Reliability Survey (July 29-2009) 197 


\section{Índice de figuras}

2.1. Espacio de Trabajo de Diagnóstico General . . . . . . . . . . 9

2.2. Clasificación de Algoritmos de Diagnóstico . . . . . . . . . 15

2.3. Esquema General de Redundancia Analítica . . . . . . . . . 18

2.4. Grafo de Ejemplo de Tanque . . . . . . . . . . . . . . . . 25

2.5. Autómata de Controlador Híbrido . . . . . . . . . . . . . . 41

2.6. Estadística de Fallos en Aeronaves . . . . . . . . . . . . . . . 42

3.1. Red de Petri . . . . . . . . . . . . . . . . 48

3.2. Red de Petri Interpretada de Control . . . . . . . . . . . . 53

3.3. Lugares y Transiciones de RdP Híbrida . . . . . . . . . . 56

4.1. Sistema HVAC . . . . . . . . . . . . . . . . . 73

4.2. Componentes del Sistema. . . . . . . . . . . . . . . . . 74

4.3. Modelo de Integración General . . . . . . . . . . . . . 75

4.4. Modelo de Integración Refinado . . . . . . . . . . . 78

4.5. Diagnosticador . . . . . . . . . . . . . . . . 80

5.1. Evolución de los Fl's a lo Largo de la Vida del Componente . . . 84

5.2. Arquitectura de Diagnóstico de Fl's . . . . . . . . . . . . 85

5.3. Variación de Diagnosticador para Fl's . . . . . . . . . 86

5.4. Rama para Diagnóstico de Fl's . . . . . . . . . . . . . . . 89

5.5. Proceso de Envasado . . . . . . . . . . . . . . . . . 93

5.6. Componentes del Sistema Proceso de Envasado. . . . . . . . . 94

5.7. Modelo de Integración General . . . . . . . . . . . . . . 95

5.8. Modelo de integración Refinado . . . . . . . . . . 97

5.9. Diagnosticador . . . . . . . . . . . . . . . 99

6.1. Sistema de Envasado de Líquidos- Híbrido . . . . . . . . . 117 
6.2. Subsistemas de Proceso de Envasado . . . . . . . . . . . . . . 121

6.3. Modelo General de RdP de Subsistema de Temperatura . . . . . . 122

6.4. Modelo General de RdP de Subsistema de pH . . . . . . . . . 122

6.5. Modelo General de RdP de Subsistema de Nivel . . . . . . . . . 123

6.6. Modelo Refinado de RdP de Subsistema de Temperatura . . . . 124

6.7. Modelo Refinado de RdP de Subsistema de pH . . . . . . . . . 124

6.8. Modelo Refinado de RdP de Subsistema de Nivel . . . . . . . . 125

6.9. Diagnosticador Híbrido Sistema de Envasado . . . . . . . . . . . 126

7.1. Helicóptero Vario UAV . . . . . . . . . . . . . . . . . 131

7.2. Sistemas que Componen un Helicóptero . . . . . . . . . . . . . 133

7.3. Motor de Combustión del Helicóptero Vario Benzin Trainer. . . . . 133

7.4. Sistema de Rotor Principal . . . . . . . . . . . . . . . 134

7.5. Sistema del Rotor de Cola. . . . . . . . . . . . . . . . . . 134

7.6. Esquema General de Adquisición de Datos HC . . . . . . . . 137

7.7. Sensor de Temperatura HC . . . . . . . . . . . . . . . . . 138

7.8. Sensor de Velocidad del Rotor . . . . . . . . . . . . . . . 138

7.9. Sensor de combustible . . . . . . . . . . . . . . . . . 138

7.10.Sensor de Vibraciones HC . . . . . . . . . . . . . . . 139

7.11.Sensor de Corriente de los Servos . . . . . . . . . . . . . . 139

7.12.Sensor Orientación - Posición . . . . . . . . . . . . . . . . . . 140

7.13.Computador Central ChipKit ．. . . . . . . . . . . . . . . . 140

7.14. Modulo transmisor Receptor XBee . . . . . . . . . . . . . . 141

7.15. Modulo Conversor Serial-USB FTDI . . . . . . . . . . . . 141

7.16. Interfaz Gráfica LabVIEW . . . . . . . . . . . . . . . . . . . . 142

7.17. Modulo DAQ LabVIEW . . . . . . . . . . . . . . . . . . . . . . . 143

7.18. Modulo Análisis y Presentación de Datos . . . . . . . . . . . 143

7.19. Modulo Red de Petri Diagnosticadora . . . . . . . . . . . . . . 144

7.20. Clasificación de los Subsistemas del Helicóptero. . . . . . . . . . 145

7.21. Modelo de RdP de componentes del Subsistema del Motor. . . . . 148

7.22. Modelo de RdP de los Componentes del Subsistema del Rotor Principal. . . . . . . . . . . . . . . . . . . . . . . 150

7.23. Modelo de RdP de los Componentes del Subsistema del Rotor de Cola . . . . . . . . . . . . . . . . . . . . . . . . . . . . 151

7.24. Modelo General de RdP del Subsistema del Motor. . . . . . . . . 152 
7.25. Modelo General de RdP del Subsistema del Rotor Principal . . . . 153

7.26. Modelo General de RdP del Subsistema del Rotor de Cola. . . . . 154

7.27. Figura 16. Modelo Refinado de RdP del Subsistema del Motor . . 160

7.28. Modelo Refinado de RdP del Subsistema del Rotor Principal . . . 161

7.29. Modelo Refinado de RdP del Subsistema del Rotor de Cola . . . . 161

7.30. Diagnosticador del Helicóptero . . . . . . . . . . . . . 163

7.31. Red de Petri Diagnosticadora . . . . . . . . . . . . . . . 167

7.32. Resumen Temperaturas vuelos . . . . . . . . . . . . . . 168

7.33. Revoluciones Vuelo Normal y Fallo del HC . . . . . . . . . . . 170

7.34.Comparación Vibraciones de Vuelo Normal y de Fallo . . . . . . 171

7.35. Vuelo Normal . . . . . . . . . . . . . . . . . . . . . . . . 174

7.36. Vuelo Fallo Vibraciones Plato . . . . . . . . . . . . . . . . 175

7.37. Vuelo Fallo Vibraciones de Cola . . . . . . . . . . . . . . . 176

7.38. Vuelo Fallo de Mezcla . . . . . . . . . . . . . . . . . . 177

7.39.Zonas de Trabajo Normal, Advertencia y Fallo de RPMS, Vibraciones y Temperatura . . . . . . . . . . . . . . . . . . 179

A.1. UAV failure average during flights in the U.S . . . . . . . . 198 


\section{Lista de Tablas}

4.1. Tabla de salidas de Sensores . . . . . . . . . . . . . . 65

4.2. Lugares RdPI del Sistema Completo . . . . . . . . . . . . . 73

4.3. Tabla de salidas de Sensores HVAC . . . . . . . . . . 76

4.4. Salidas de la Tabla de Integración de Sensores HVAC . . . . . 76

4.5. Tabla de Integración de Sensores HVAC . . . . . . . . . . . 77

5.1. Lugares RdPI del Sistema Completo . . . . . . . . . . . . . 94

5.2. Tabla de salidas de Sensores Envasado . . . . . . . . . . . . 96

5.3. Salidas de la Tabla de Integración de Sensores Envasado . . . . 96

5.4. Tabla de Integración de Sensores Envasado . . . . . . . . . . . 96

6.1. Tabla de salidas de Sensores . . . . . . . . . . . . . . . 108

6.2. Salidas de la Tabla de Integración de Sensores Subsistema . . . . 109

6.3. Tabla de Integración de Sensores Subsistema . . . . . . . . . 109

7.1. Características del Helicóptero Robbe . . . . . . . . . . . 133

7.2. Conjunto Discreto de Salidas de Sensores de los Subsistemas del HC . . . . . . . . . . . . . . . . . . . . . . 156

7.3. Tabla de Integración de Sensores - Adición de Lugares de Salida Motor. . . . . . . . . . . . . . . . . . . . . . . . 157

7.4. Tabla de Integración de Sensores - Adición de Lugares de Salida Rotor Ppal. . . . . . . . . . . . . . . . . . . . . . 157

7.5. Tabla de Integración de Sensores - Adición de Lugares de Salida Rotor Cola. . . . . . . . . . . . . . . . . . . . . . . . . . . 158

7.6. Tabla de Integración de Sensores - Motor. . . . . . . . . . . . 159

7.7. Tabla de Integración de Sensores - Plato. . . . . . . . . . . 159

7.8. Tabla de Integración de Sensores del Rotor de Cola. . . . . . . 160

7.9. Resumen de Misiones . . . . . . . . . . . . . . . . . . . . . . 164 
7.10. Tabla de Verdad de Interacción de la $T / F / R P M / G \ldots \ldots 166$

7.11. Measured Variables and Thresholds in UAV . . . . . . . . . . 172

7.12. Resumen Número de Fallos de Variables en Misiones . . . . . . 173

7.13.Comportamiento del HC en Vuelo Normal . . . . . . . . . . . . 174

7.14.Comportamiento del HC en Vuelo con Fallo de Vibraciones de Plato175

7.15. Comportamiento del HC en Vuelo con Fallo de Vibraciones de Cola176 7.16. Comportamiento del HC en Vuelo con Fallo de Vibraciones de Cola177

7.17.Zonas de Trabajo para RPMs, Vibraciones, Temperatura . . . . . 178 


\section{Nomenclatura}

AESMF Adaptive Extended Set-Member Filter

FD Diagnóstico de Fallos

FDI Detección y Aislamiento de Fallos

Fl's Fallos Intermitentes

FTC Control Tolerante a Fallos

HC Helicóptero

HVAC Heating, Ventilation and Air Condition

KF Kalman Filter

KFE Filtros de Kalman Extendido

LES Mínimos Cuadrados Parcial

LPV Linear Parameter Varying

LTI Linear Time-Invariant

MEF's Máquinas de Estado Finitas

NN Neural Networks

OKID Observer/Kalman Filter Identification

PCA Análisis de Componentes Principal

PLS Mínimos Cuadrados Parciales

QTA Análisis de Tendencia Cualitativa 
LISTA DE TABLAS

RdP Redes de Petri

RdPD Red de Petri Diagnosticadora

SDG Signed Digraphs

SED's Sistemas de Eventos Discretos

SPC Control de Procesos Estadísticos

UAS Sistemas Aéreos no Tripulados

UAVs Vehículos Aéreos no Tripulados

UHC Helicóptero no Tripulado

UIO Entrada no Conocida

UKF Filtros de Kalman Unscented 


\section{Capítulo 1}

\section{Introducción}

\subsection{Motivación}

Las Plantas de fabricación y sus equipos de investigación hacen grandes esfuerzos para que sus procesos sean cada vez más seguros y confiables, se centran en la seguridad del coste económico y el bienestar de los operadores. En el área económica, es importante reducir los costos de paradas por mantenimiento y las pérdidas ocasionadas por el fallos en los plazos de entrega. Por otra parte, la seguridad de los operadores es en ocasiones más relevantes, debido a los problemas legales asociados, por ejemplo, cuando hay accidentes de trabajo que ponen en riesgo la vida de los operadores. Por lo tanto, el diagnóstico de fallos no sólo se ha convertido en una fuente de inversión para la industria, si no también para los grupos de investigación que están interesados en la generación de las contribuciones al campo de la detección y aislamiento de fallos. La temprana detección de fallos es crítica en evitar una degradación del comportamiento. El Diagnóstico preciso ayuda a tomar decisiones correctas en acciones de emergencia y reparación. Las técnicas de Diagnostico de Fallos (FD) se han desarrollado dentro de una mayor área de investigación, en la intersección de sistemas de ingeniería de control, Inteligencia Artificial, aplicaciones Matemáticas y Estadísticas, y en tales campos de aplicación como Química, Eléctrica, Mecánica e Ingeniería Aeroespacial. Por ello existe un alto interés y necesidad de que todos los sistemas tengan inmerso una aplicación de Diagnóstico de Fallos. La actividad de diagnóstico abarca una variedad de daños tales como: fallo y degradación de unidades de proceso, parámetros no 
deseados, entre otros, y cada vez es más complicado de implementar debido al tamaño y complejidad de los sistemas.

Las Redes de Petri (RdP) son una herramienta de modelado gráfico y matemático aplicada a muchos sistemas. Es una herramienta con mucha proyección en el campo de la automática, en la cual se puede estudiar y describir información de sistemas de procesamiento que son caracterizados por ser concurrentes, paralelos, asíncronos, distribuidos, no determinísticos y/o estocásticos. Como herramienta gráfica las RdP puede ser usada como una ayuda de comunicación visual, similar a las cartas de flujo, diagramas de bloques y redes. En adición las marcas son usadas en estas redes para simular la dinámica y actividades de concurrencia de sistemas. Como herramienta matemática es posible hacer ecuaciones de estado, ecuaciones algebraicas y otros modelos que representan el comportamiento de sistemas.

Un mercado civil con un creciente número de aplicaciones potenciales de Sistemas Aéreos No Tripulados (UAS) está emergiendo, y la promoción de actividades de investigación está jugando un papel importante para lograr la integración deseada de los UAS con escenarios civiles. Hay una enorme distancia entre los grandes y costosos sistemas militares, que son capaces de transportar equipos redundantes y un completo sistema de monitoreo, y los pequeños, ligeros y baratas aeronaves comerciales de bajo coste. Por esto, aunque hay varias iniciativas buscando incluir los vehículos aéreos no tripulados (UAVs) en el espacio aéreo no segregado, usando un único sistema de control de tráfico aéreo, estos estudios son no aplicables para pequeñas aeronaves comerciales. Durante los últimos años, diferentes organizaciones [26] han contribuido con esfuerzos sobresalientes con el fin de introducir un marco regulador necesario que permita la integración completa y transparente de los UAS dentro de las actividades civiles. De acuerdo con estos esfuerzos, los grupos de investigación que participan en actividades con UAVs están preocupados, no sólo por la frecuencia e incidencia de los accidentes causados por fallas en los UAS sino también por la vigilancia y seguridad de la población, mientras se realice cualquier tarea en entornos civiles. Además, aunque existen avances en resolver este problema, Europa debe continuar identificando las causas e incluir en la regulación los fundamentos y rutinas necesarios para el uso de la UAS, a fin de disminuir los porcentajes de fallo. 
Este crecimiento y las nuevas tendencias legislativas han requerido la ampliación de las técnicas de Diagnóstico de fallos (FD) en el campo de los UAS. Además, no sólo la experiencia de cualquier equipo de investigación que trabaja con UAS, sino también los datos reportados por Freeman [32], sobre la fiabilidad de vehículos aéreos no tripulados (referido a las aeronaves individualmente) muestran que son muy vulnerables a las situaciones imprevistas. Las fuentes de esos problemas pueden provenir de los dispositivos de equipo (estación de control y aeronave) o cuestiones operativas, destacando de esta manera la falta de métodos para evitar que las operaciones del piloto averíen el sistema.

Aunque los sistemas multirotor (Quarotor y Hexarotor) recientemente han entrado en el mercado en la categoría de mini-UAVs basados en su simplicidad, los helicópteros $(\mathrm{HC})$ son aún las plataformas más utilizadas cuando se requiere maniobras de sostenimiento con una capacidad de carga mas elevada. Este tipo de aeronave es inherentemente inestable y exhibe una dinámica rápida. Los helicópteros no tienen las propiedades de degradación sutiles de aviones o aeronaves de ala fija en caso de fallos. Incluso con la mejora de dispositivos de aumento de estabilidad, se requiere un experimentado piloto para su control durante el vuelo. Por lo tanto, un helicóptero ha sido usado para aplicar esta investigación, considerando que los helicópteros son la más compleja plataforma desde un punto de vista mecánico. De ahí, que un fallo en cualquier parte de los sensores, actuadores o sistema de control del helicóptero puede llevar a una situación peligrosa.

\subsection{Objetivos y Alcance}

El objetivo de este trabajo de investigación es contribuir en la solución de los inconvenientes mencionados previamente. En la mayoría de los accidentes en los procesos industriales y especialmente con aeronaves no tripuladas se desconoce sus causas, adicionalmente se generan perdidas que van desde lo económico hasta el tiempo gastado en los desarrollos. Por lo anterior, las investigaciones presentadas en esta tesis ayudará tanto a los grupos de investigación y usuarios de las UAVs, como a cualquier proceso industrial que necesite de una herramienta de diagnóstico de fallos. Los sistemas híbridos en la práctica necesitan herramientas de FD fáciles de implementar, por ello gracias a los recursos 
que ofrecen las redes de petri interpretadas, se construye un diagnosticador con capacidad de detectar fallos intermitentes y definitivos.

El trabajo de investigación debe ser validado a través de pruebas reales, la red de petri diagnosticadora desarrollada e implementada en el helicóptero no tripulado, ha sido sometida a vuelos reales en condiciones de funcionamiento normal y de fallo. Por lo anterior, los objetivos que se enmarcan en la presente tesis son los siguientes:

- Presentar un análisis detallado de los trabajos previos en diagnóstico de fallos, desde un punto de vista general hasta el tema específico de los sistemas aéreos no tripulados.

- Diseño e implementación del procedimiento completo de una herramienta de FD construida con Redes de Petri Interpretadas ( $R d P)$ para construir un diagnosticador, capaz de detectar fallos permanentes e intermitentes.

- Diseño de un sistema de adquisición de datos, encargado de albergar el diagnosticador y probar la herramienta de FD.

- Implementación de la herramienta en varios sistemas industriales, incluido un helicóptero no tripulado, evaluado en condiciones de vuelo normal y de fallo.

- Recopilación de información que permite conocer el funcionamiento del helicóptero, de cara a desarrollar investigaciones futuras, entre las cuales están estrategias de control, análisis de comportamiento de las variables, entre otros.

Como una estrategia previa al desarrollo de la herramienta de diagnóstico de fallos, se hacía indispensable conocer en detalle los principales fuentes de fallo en los UAVs. Para ello, a parte de las fuentes bibliográficas disponibles, se decidió consultar a los grupos de investigación registrados en la organización UVS International, buscando a través de un estudio de fiabilidad (Apéndice A), información relevante de las misiones que realizan y cuales son los accidentes o problemas encontrados en sus misiones. Aunque inicialmente el estudio fue recibido con beneplácito, al final, no se pudo desarrollar por inconvenientes de la organización que desconocemos. 


\subsection{Principales Contribuciones de la Tesis}

Las aportaciones en esta tesis están desarrollas desde el diseño teórico de la herramienta hasta su implementación práctica.

1. Herramienta para el diagnóstico de fallos intermitentes y definitivos de sistemas de eventos discretos.

2. Herramienta para el diagnóstico de fallos intermitentes y definitivos de sistemas híbridos.

3. Identificación de variables y sus umbrales de funcionamiento normal y de fallo de un helicóptero no tripulado.

4. Desarrollo de herramientas y espacio de trabajo para las pruebas de campo del diagnosticador en condiciones reales.

5. Recopilación de datos y análisis de comportamiento del helicóptero no tripulado, volando en condiciones normal y de fallo.

Los resultados obtenidos de esta investigación han sido presentados en congresos internacionales y publicadas en revistas científicas de renombre: en resumen, 1 revista recogida en el JCR[94], 7 congresos [93][96][92][97][98][99][95], 1 Capítulo de libro [91]. En la fecha de la publicación de la presente tesis, varias comunicaciones están en revisión en revistas incluidas en el JCR.

\subsection{Organización de la Tesis}

Esta sección explica como está organizada ésta tesis. El documento está compuesto por 8 capítulos, Después de hacer una revisión de los antecedentes, motivación y alcance en el capítulo 1, a continuación se describe el contenido de la investigación. El capítulo 2 es dedicado a una amplia revisión de los trabajos previos en diagnóstico de fallos partiendo desde un punto de vista generalizado (clasificación de los algoritmos según el conocimiento a priori), posteriormente se hace una clasificación en las técnicas implementadas en sistemas de eventos discretos, para finalmente presentar las investigaciones en el área de las aeronaves no tripuladas. En el capítulo 3 se encuentran los fundamentos teóricos relacionados con las RdP, teoría utilizada para la construcción del diagnosticador. Los dos capítulos previos ayudan al lector a entender la herramienta desarrollada en esta investigación. 
En el capítulo 4 se describe la herramienta de FD, inicialmente fue planteada para aplicar a sistemas de eventos discretos, se muestra el proceso de construcción del modelo y diagnosticador utilizando RdP. Para poder incluir en la herramienta de diagnóstico a los fallos intermitentes, es necesario agregar la capacidad de evaluación de la dinámica temporal en el diagnosticador, el cual es presentado en el capítulo 5. En estos capítulos también se desarrollan aplicaciones teóricas de la herramienta, implementados en un sistema de HVAC (Heating, Ventilation and Air Condition) y en un sistema de envasado de líquidos. La principal contribución de esta tesis está incluida en los capítulos 6 y 7 . Por ello, todo el proceso de construcción sistemática del diagnosticador es representado en el capítulo 6; define paso a paso cada uno de los procedimientos a realizar para construir el diagnosticador en sistemas híbridos. En el capítulo 7 se muestra la aplicación de la herramienta de diagnóstico de fallos a un helicóptero no tripulado, describiendo las etapas ejecutadas para realizar pruebas reales de vuelo, entre las cuales están el diseño del sistema de adquisición de datos, diseño de la herramienta de software, entre otras. Finalmente, aquí se muestran los resultados obtenidos en los vuelos de comportamiento normal y de fallo de la aeronave. Por último en el capítulo 8 se cita la conclusión del trabajo de investigación. 


\section{Capítulo 2}

\section{Trabajos Previos en Diagnóstico de Fallos}

Debido al amplio alcance de los problemas de diagnóstico de fallos y las dificultades de estos en soluciones de tiempo real, varias investigaciones basadas en ordenador han sido desarrolladas en los últimos años. Ellas cubren una amplia variedad de técnicas tales como los esfuerzos tempranos usando árboles de fallos y grafos (digraphs), investigaciones analíticas, sistemas basados en conocimiento y redes neuronales en estudios mas recientes. Desde una perspectiva de modelado, hay métodos que requiere modelos de procesos precisos, semi-cuantitativos o cualitativos. Por otro lado del espectro, hay métodos que no asumen cualquier forma de la información del modelo y cuentan solo con información de la historia del proceso. En adición, dado el conocimiento del proceso, hay diferentes técnicas que puede ser aplicadas a realizar diagnóstico.

El ánimo básico de este capítulo es suministrar un comparativo estudio de varios métodos de diagnóstico y diferentes perspectivas, como los planteados en los documentos [103][83], se muestra una perspectiva de como estos diferentes métodos se relacionan o difiere de cada otro, se comentan distintas investigaciones, ventajas y desventajas, no se pretende discutir cada técnica en detalle, pero se quiere aportar conceptos generales que busquen orientar hacia las referencias de los investigadores del diagnóstico de fallos (FD).

En el presente capítulo se pretende abordar en primer lugar algunas definiciones y nomenclatura usada en el área del diagnóstico de fallos de procesos, buscando tener elementos de juicio al revisar la clasificación de las diferentes 
técnicas de diagnóstico de fallos, se presentan las características deseadas en un sistema de diagnóstico de fallos y posteriormente se estudian las técnicas de diagnóstico de fallos basada en modelos cuantitativos, en modelos cualitativos, basados en históricos del proceso; seguido se presentan algunas técnicas mas específicas en el marco del diagnóstico de fallos de los sistemas de eventos discretos e híbridos. Para finalizar se hace un análisis de los trabajos previos en el diagnóstico de fallos dedicado a las sistemas aéreos no tripulados (Unmanned Aerial System - UAS)

\subsection{Conceptos en Diagnóstico de Fallos}

Es importante dar a conocer conceptos fundamentales en el diagnóstico de fallos antes de evaluar las diferentes técnicas existentes, el término fallo es generalmente definido como una salida de un rango aceptable de una variable observada o un parámetro calculado asociado con un proceso [60]. Esto define a un fallo como un proceso de anormalidad o síntoma. tal como una alta temperatura en un reactor o baja de calidad de un producto, etc. La causa de esta anormalidad, como un fallo de la bomba refrigerante o el controlador, es(son) llamado el evento(s) básico de la base de la causa(s). El evento básico es también referido como una mal función o avería. Desde que se vea la tarea de diagnóstico como un problema de clasificación, el sistema de diagnóstico es también referido como un clasificador de diagnóstico, en la figura 2.1 se describen los componentes de espacio de trabajo de diagnóstico de fallos, se muestra un sistema de un proceso controlado y se indican las diferente fuentes de fallo que se dan en el.

\subsubsection{Clases de Fallos de un Sistema}

En general en los procesos industriales se trata con tres clases de fallos o averías que se describen a continuación.

\section{Cambios Bruscos de Parámetros en el Modelo.}

En cualquier modelado, hay procesos corriendo bajo el nivel seleccionado de detalles del modelo. Estos procesos los cuales no son modelados son típicamente agrupados como parámetros e incluyen interacciones de los límites del 


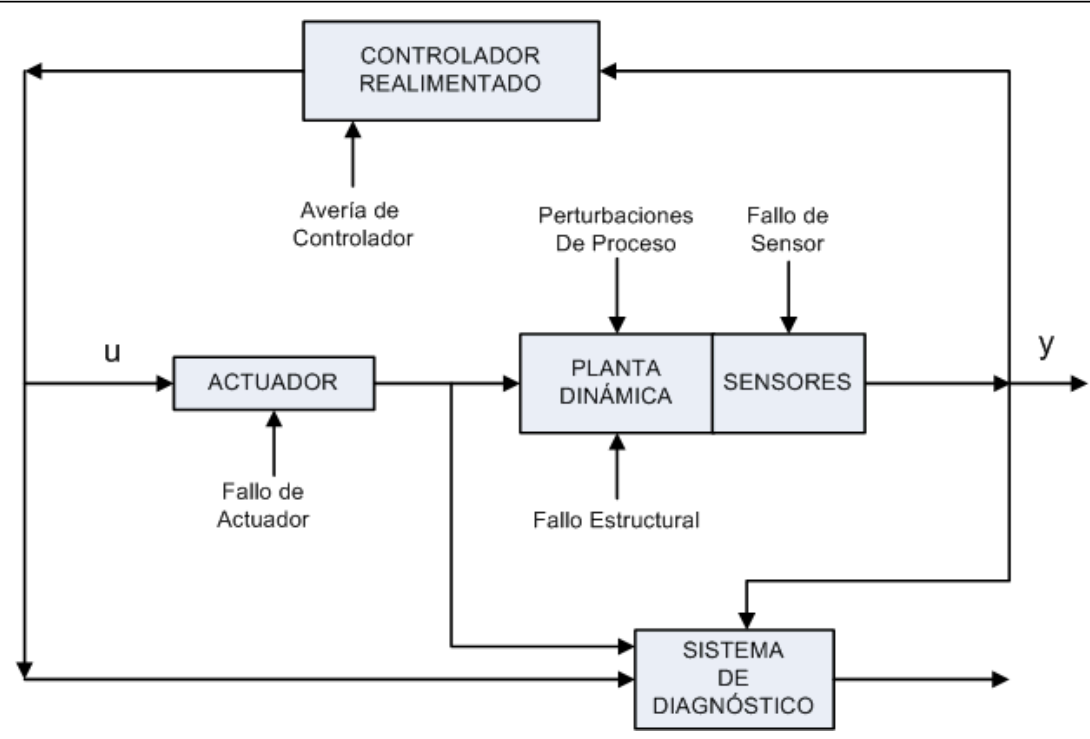

Figura 2.1: Espacio de Trabajo de Diagnóstico General

sistema. Los parámetros fallan incrementándose cuando hay entrada de perturbaciones en el proceso, desde el ambiente a través de una o mas variables independientes. Un ejemplo de un malfuncionamiento es un cambio en la concentración del reactivo en el estado normal o estable en un alimentador del reactor. Aquí, la concentración es una variable independiente (exógena), una variable de quien la dinámica no es suministrada con el proceso. Otro ejemplo es el cambio en el coeficiente de la transferencia de calor debido al fallo en el intercambiador de calor.

\section{Cambios Estructurales}

Cambios estructurales se refiere a cambios en el propio proceso. Ellos ocurren debido a fallos en el equipamiento. Los fallos estructurales resultan debido a un cambio en el flujo de información entre varias variables. Para manejar una avería de este tipo en un sistema de diagnóstico habría que requerir el levantamiento de las ecuaciones del modelo apropiadamente y reestructurar las otras ecuaciones en orden a describir la situación actual del proceso. Un ejemplo de un fallo estructural podría ser el fallo de un controlador, otro ejemplo incluye una válvula atascada, una tubería goteando o rota, etc. 


\section{Malfuncionamiento de Sensores y Actuadores}

Errores graves usualmente ocurren con actuadores y sensores. Estos pueden ser debido a fallos fijos, una desviación constante (positiva o negativa) o un fallo fuera de rango. Algunos de los instrumentos suministran señales de retorno las cuales son esenciales para el control de la planta. Un fallo en uno de los instrumentos puede causar que las variables de estado de la planta se desvíen a valores mas allá de los permitidos a menos que el fallo sea detectado rápidamente y las acciones de corrección sean tomadas a tiempo. Este es el propósito del diagnóstico, detectar rápidamente cualquier fallo en los instrumentos el cual podría seriamente degradar el funcionamiento del sistema de control.

Fuera del alcance del diagnóstico de fallos son las incertidumbres no estructuradas, ruidos de proceso y ruido en las medidas. Incertidumbre en las medidas son principalmente fallos que no son modelados a priori. Ruidos de proceso se refieren a la desigualdad entre el proceso actual y las predicciones de las ecuaciones del modelo, considerando, que el ruido en las medidas se refieren al componente aditivo de alta frecuencia en las medidas del sensor.

\subsubsection{Características Deseables de un Sistema de Diagnóstico de Fallos}

En orden a evaluar varias investigaciones de diagnóstico, es útil identificar un conjunto de características deseables que un sistema de diagnóstico debe poseer. De esta manera, las diferentes investigaciones puede ser evaluadas contra un conjunto común de requerimientos. Aunque éstas características no sean usualmente reunidas por cualquier solo método de diagnóstico, ellas son útiles para referencia de varios métodos en términos de información a priori que necesita ser suministrada, confiabilidad de solución, generalidad y eficiencia en computación etc. En este contexto se necesita entender conceptos importantes, integridad y resolución, antes precediendo a las características de un buen clasificador de diagnóstico. Cuando ocurra una anormalidad en un proceso, en general el clasificador de diagnóstico debería de surgir con un conjunto de hipótesis o fallos que expliquen la anormalidad. La integridad de un clasificador de diagnóstico exigiría que el fallo actual sean un subconjunto del conjunto de fallos propuestos. Resolución de un clasificador de diagnóstico exigiría que el conjunto de fallos sea tan mínimo como sea posible. Así, hay una compensa- 
ción entre integridad y resolución. La compensación está en la precisión de las predicciones. Estos dos conceptos se repetirían cuando diferentes clasificadores diseñados sean comparados. A continuación se presentan un conjunto de características deseables que el sistema de diagnóstico debería poseer.

\section{Detección y Diagnóstico Rápido (Quick Detection and Diagnosis)}

El sistema de diagnóstico debe responder rápidamente a los malfuncionamientos del proceso. Sin embargo, respuesta rápida a tolerancia y diagnóstico de fallos realizado durante la operación normal son dos metas en conflicto [106]. Un sistema que es diseñado para detectar un fallo (cambios abruptos particulares) rápidamente estaría sensible a influencias de altas frecuencias. Esto hace el sistema sensible a ruidos y puedan llevar frecuentemente a falsas alarmas durante la operación normal, el cual puede ser perjudicial.

\section{Aislabilidad (Isolability)}

Aislabilidad es la habilidad de un sistema de diagnóstico a distinguir fallos diferentes. Bajo condiciones ideales libre de ruidos e incertidumbres de modelado, esto asciende a decir que el clasificador de diagnóstico debe estar disponible para generar salidas que sean ortogonal al fallo que ha ocurrido. Por supuesto que la habilidad de diseñar clasificadores aislables depende del gran tamaño en las características del proceso. Hay también un intercambio entre la aislabilidad y el rechazo de las incertidumbres de modelado. Muchos de los clasificadores trabajan con varias formas de información redundante y así hay un solo grado de libertad limitado para el diseño del clasificador. Debido a esto, un clasificador con alto grado de aislabilidad debería usualmente hacer un pobre trabajo en rechazo de incertidumbres de modelado y viceversa.

\section{Robustez (Robutsness)}

El sistema de diagnóstico debería ser robusto a ruidos e incertidumbres. El rendimiento debería degradarse gradualmente en vez de fallo total y abrupto. La robustez precluye pruebas de aislabilidad determinística donde el umbral sea colocado cerca de cero. En la presencia de ruido, este umbral puede tener 
que ser elegido conservativamente. Así, como se dijo anteriormente, la robustez necesita ser balanceada con el rendimiento.

\section{Identificabilidad Novedosa (Novelty Identifiability)}

Uno de los requerimientos mínimos de un sistema de diagnóstico es que sea capaz de decidir, dadas las condiciones corrientes del proceso, si el proceso esta operando normalmente o anormalmente, y si es anormal, si la causa es un malfuncionamiento conocido o no conocido. Este criterio es conocido como identificabilidad novedosa. En general, suficientes datos pueden estar disponibles para modelar el comportamiento normal del proceso. Sin embargo, típicamente no tiene tales datos históricos de proceso disponibles para modelar las regiones anormales satisfactoriamente. Solo un poco de datos patrones puede estar disponibles cubriendo porciones de una región anormal. Así, es posible que muchas de las regiones de operación anormal puede no haber sido modeladas adecuadamente. Esto propondría grandes cambios en alcanzar la identificabilidad novedosa. Incluso bajo estas condiciones difíciles, el sistema de diagnóstico debería estar disponible a reconocer la ocurrencia de fallos nuevos y no clasificar erróneamente ellos como uno de otro fallo conocido o como operación normal.

\section{Clasificación de la Estimación de Error (Classification error estimate)}

Un importante requerimiento para un sistema de diagnóstico esta en construir la confianza del usuario en esta confiabilidad. Esto podría ser muy fácil si el sistema de diagnóstico pudiera suministrar una estimación a priori en clasificación de error que pueda ocurrir. Tales medidas de error serían útiles para proyectar niveles de confianza en las decisiones de diagnóstico por el sistema, dando al usuario una buena percepción para la confiabilidad de las recomendaciones por el sistema.

\section{Adaptabilidad (Adaptability)}

Los procesos en general cambian y evolucionan debido a cambios en las entradas externas o a cambios estructurales. Los procesos en condiciones de 
operación pueden cambiar no solo debido a perturbaciones, pero también debido a cambios en las condiciones del ambiente tales como: cambios en las cantidades de producción con cambios de demandas, cambios en la calidad de la materia prima etc. Así, el sistema de diagnóstico debe ser posible desarrollar el alcance gradualmente del sistema como nuevos casos y problemas emerjan, como mas información esté disponible.

\section{Facilidad de Explicación (Explanation Facility)}

Además de la habilidad para identificar la fuente de un malfuncionamiento, un sistema de diagnóstico debe también suministrar explicaciones en como el fallo fue originado y propagado en la situación corriente. Esto es un factor muy importante de diseño de sistemas que soportan decisión on-line. Esto requiere la habilidad para razonar acerca de la relación entre causa y efecto en un proceso. Un sistema de diagnóstico tiene que justificar estas recomendaciones, así que el operador pueda acordemente evaluar y actuar usando su experiencia. El sistema de diagnóstico debería no solo justificar porque ciertas hipótesis fueron propuestas, sino también explicar porque ciertas otras hipótesis no fueron propuestas.

\section{Requerimientos de Modelado (Modelling Requirements)}

La cantidad de modelado requerido para el desarrollo de un clasificador de diagnóstico es una importante cuestión. Para el rápido y fácil despliegue de clasificadores de diagnóstico de tiempo real, los esfuerzos de modelado deben ser tan mínimo sea posible.

\section{Requerimientos Computacionales y Almacenamiento (Store and Compu- tational Requierements)}

Usualmente, soluciones rápidas de tiempo real deben requerir algoritmos e implementaciones las cuales sean computacionalmente menos complejas, pero puede suponer altos requerimientos de almacenamiento. Se preferiría un sistema que esté disponible para alcanzar un razonable balance entre estos dos requerimientos compitiendo. 


\section{Identificabilidad de Fallos Múltiples (Multiple Fault Identifiability)}

La habilidad para identificar fallos múltiples es un importante pero difícil requerimiento. Esto es un problema difícil debido a la interacción natural de muchos fallos. En un sistema general no lineal, las interacciones deben usualmente ser sinérgicas y así un sistema de diagnóstico, no puede estar disponible para usar los patrones de fallos individual para modelar los efectos combinados de los fallos. Por otro lado, enumerando y diseñando separadamente para varias combinaciones de fallos múltiples, deben convertirse en combinatoriamente prohibida para grandes procesos.

\subsection{Clasificación de los Algoritmos de Diagnóstico según el Conocimien- to a Priori}

Como es conocido dos de los principales componentes en un clasificador de diagnóstico son: (i) el tipo de conocimiento y (ii) el tipo de estrategia de búsqueda de diagnóstico. La estrategia de búsqueda de diagnóstico es una muy fuerte función del esquema de representación del conocimiento el cual a su vez es principalmente influenciada por la clase de conocimiento a priori disponible. Así, el tipo de conocimiento a priori usado es el más importante característica distinguible en sistemas de diagnóstico. por ello, en este documento la clasificación de los sistemas de diagnóstico se basa en el conocimiento a priori utilizado.

Lo básico del conocimiento a priori que es necesario para diagnóstico de fallos es el conjunto de fallos y la relación entre las observaciones (síntomas) y los fallos. Un sistema de diagnóstico puede tenerlos explícitamente o éste puede ser inferido de alguna fuente de dominio del conocimiento. El dominio de conocimiento a priori puede ser desarrollado de un entendimiento fundamental del proceso usando entendimiento de primer principio. Tal conocimiento es referido como profundo, causal o conocimiento basado en modelos [65]. Por otro lado, ésta se puede deducir de la experiencia pasada con el proceso. Este conocimiento es referido como superficial, compilado, evidencial o conocimiento basado en procesos de Históricos.

El conocimiento a priori basado en modelos puede ser ampliamente clasificado como cualitativo o cuantitativo. El modelo es usualmente desarrollado basado en algún entendimiento fundamental de la física del proceso. En modelos cuantitativos este entendimiento es expresado en términos de relaciones fun- 


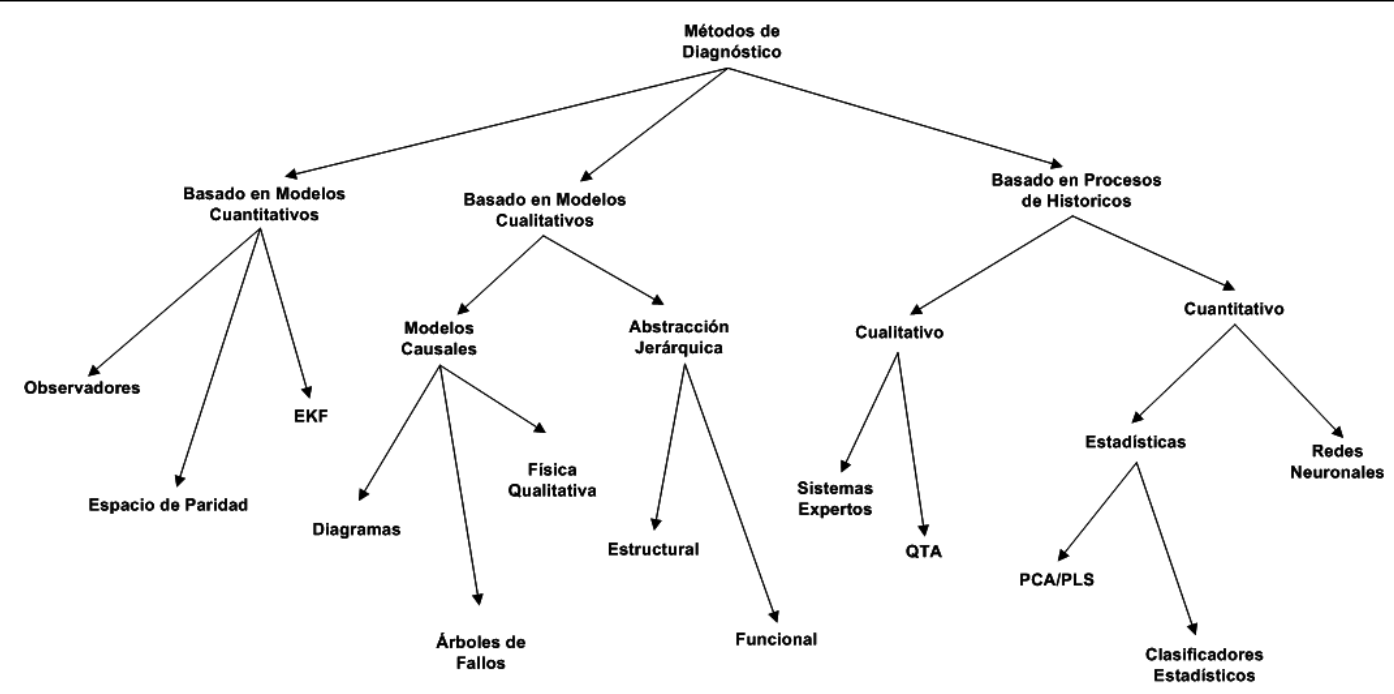

Figura 2.2: Clasificación de Algoritmos de Diagnóstico

cionales matemáticas entre las entradas y salidas del sistema. En contraste, en modelos cualitativos estas ecuaciones de relación son expresadas en términos de funciones centradas alrededor de diferentes unidades en un proceso.

En contraste a las investigaciones basadas en modelos donde un conocimiento a priori acerca del modelo (entre cuantitativo o cualitativo) del proceso es asumido, en métodos basado en históricos solo la disponibilidad de una gran cantidad de datos de proceso históricos es asumido. Hay diferentes caminos en el cual estos datos puede ser transformados y presentados como conocimiento a priori para un sistema de diagnóstico. Esto es conocido como la extracción de características del proceso de los datos históricos del proceso, y es hecho para facilitar el diagnóstico posterior. Este proceso de extracción puede principalmente proceder como entre característica de extracción cuantitativa o cualitativa. En característica de extracción cuantitativa se puede realizar entre una extracción de características estadísticas o no estadísticas. esta clasificación de sistema de diagnostico es mostrada en le figura 2.2.

Para aclarar un poco, está claro que todos los modelos necesitan datos para estimar algunos de los parámetros en el modelo y todos los métodos basados en datos de procesos necesitan para extraer de alguna forma el modelo para realizar diagnóstico de fallos. la clasificación de cuantitativo, cualitativo e histórico de procesos es según el punto de vista de [103], es una clasificación en términos de la manera en la cual estos métodos investigan el problema de diagnóstico de fallos. Como un ejemplo, aunque los modelos para observadores 
(clasificado como cuantitativo) sea basado en datos de entrada-salida, el uso de éstos modelos en generación de diagnóstico resulta principalmente siguiendo una investigación cuantitativa. Sin embargo, una investigación cualitativa tal como Simulación Cualitativa (Qualitive Simulation QSIM) de nuevo basada en un modelo (modelo cuantitativo de primer principio) usa un espacio de trabajo cualitativo distinto para la generación de la explicación de diagnóstico. Similarmente, investigaciones en Redes Neuronales para diagnóstico de fallos principalmente han sido investigadas de un punto de vista de reconocimiento de patrones y así se tiene clasificado estas investigaciones bajo métodos de histórico de procesos, aunque ellos están directamente relacionados a modelos de espacio estado. Se cree que a pesar del traslape esta es una buena clasificación para estrategias de detección y aislamiento de fallos (FDI).

\subsubsection{Algoritmos Basados en Modelos Cuantitativos}

Muchos de los trabajos basados en modelos cuantitativos han sido basados en modelos generales de espacio estado y entradas-salidas, como se discutió anteriormente. Sin embargo, hay una amplia variedad de tipos de modelos cuantitativos que han sido considerados en diagnóstico de fallos tales como modelos de primer principio, modelos de respuesta en frecuencia, entre otros. Los modelos de primer principio no han sido muy populares en diagnóstico de fallos por la complejidad computacional en utilizar estos modelos en sistemas de diagnóstico de tiempo real y la dificultad en desarrollar estos modelos. La clase más importante de modelos que han sido duramente investigadas en estudios de diagnóstico de fallos son los modelos de espacio estado o entrada-salida y así el enfoque de este resumen es en estos tipos de modelos.

\section{Redundancia Analítica}

En el área de control automático, los problemas de detección de fallos son conocidos como FDI basado en modelos, contando con un modelo explícito del monitoreo de la planta, todos los métodos FDI basado en modelos (y muchos de los métodos de diagnóstico estadístico) requiere dos pasos. El primer paso genera inconsistencias entre el comportamiento actual y el esperado. Tales inconsistencias, también llamadas residuos, son señales artificiales que refle- 
jan los fallos potenciales del sistema. El segundo paso selecciona una regla de decisión para diagnóstico.

El chequeo para la inconsistencia necesita alguna forma de redundancia. Hay dos tipos de redundancias, redundancia de hardware y redundancia analítica. el primero requiere sensores redundantes. Éste ha sido utilizado en el control de tales sistemas de seguridad crítica como vehículos de aeronaves espaciales y plantas de energía nuclear. Sin embargo, esta aplicación es limitada debido al costo extra y el espacio adicional requerido. Por otro lado, Redundancia analítica (también llamada redundancia funcional, inherente o funcional) es alcanzada de la dependencia funcional entre las variables del proceso y es usualmente suministrada por un conjunto de relaciones algebraicas o temporales entre los estados, entradas y las salidas de un sistema. Acorde a como la redundancia es lograda, redundancia analítica puede ser clasificada en dos categorías, directa y temporal.

Una redundancia directa es lograda de relaciones algebraicas entre medidas diferente de sensor. Tales relaciones son útiles en computar el valor de la medida de un sensor de medidas de otro sensor. El valor computado es entonces comparado con el valor medido de ese sensor. Una discrepancia indica que un fallo de sensor puede haber ocurrido. Una redundancia temporal es obtenida de una relación diferencial entre salidas de sensor y entradas de actuador. Con datos de entrada y salida del proceso, redundancia temporal es útil para detección de fallos de sensor y actuador.

Un esquema general usando redundancia analítica en sistemas de diagnóstico es mostrado en la 2.3. La esencia de la redundancia analítica en diagnóstico de fallos es chequear la coherencia del comportamiento del sistema actual contra el modelo del sistema. Cualquier inconsistencia expresada como residuos puede ser usada para propósitos de diagnóstico y aislamiento de fallos. Los residuos deben estar cerca a cero cuando no ocurren fallos, pero muestran valores significantes cuando el comportamiento del sistema cambia. La generación de los residuos de diagnóstico requiere un modelo matemático explícito del sistema. Puede ser usado un modelo derivado analíticamente usando primer principio o un modelo de caja negra obtenido empíricamente. También, métodos estadísticos son usados frecuentemente para toma de decisión.

Los métodos basados en redundancia analítica derivan residuos los cuales son insensibles a incertidumbres pero son sensibles a fallos. Uno de los caminos 


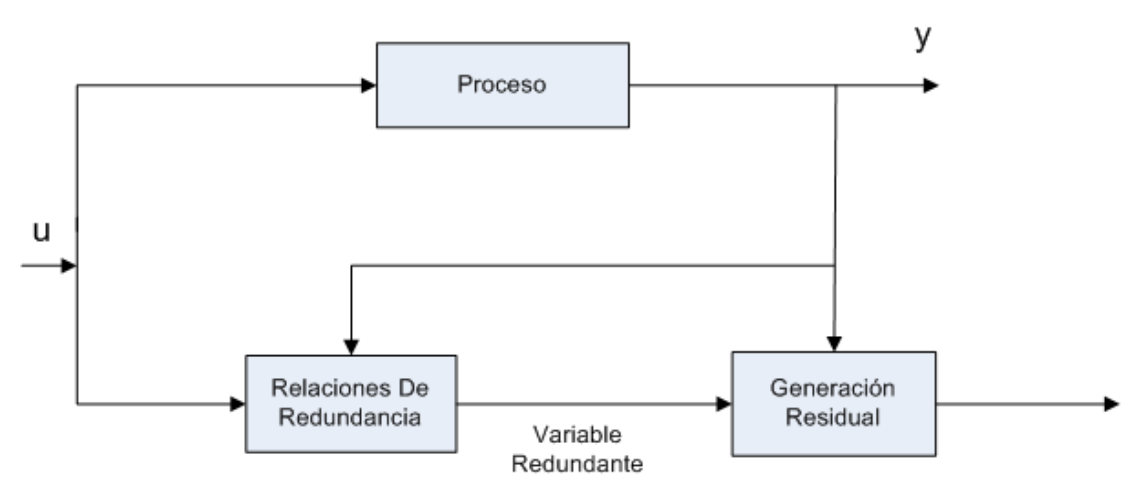

Figura 2.3: Esquema General de Redundancia Analítica

populares de hacer esto es el método de desacople de incertidumbre. En este documento, todas las incertidumbres son tratadas como perturbaciones y filtros son diseñados para desacoplar los efectos de fallos y entradas no conocidas que también puede ser diferenciadas [31].

\section{Generación Residual en Sistemas Dinámicos}

El esquema de redundancia analítica para diagnóstico de fallos son básicamente técnicas de procesamiento de señales usando estimación de estados, estimación de parámetros, filtrado adaptativo, entre otros.

Observadores de Diagnóstico para Sistemas Dinámicos La principal inquietud del FDI basado en observadores es la generación de un conjunto de residuos los cuales detectan e identifican singularmente diferentes fallos. Estos residuos deben ser robustos en el sentido que las decisiones no sean corruptas por tales entradas no conocidas como incertidumbres no estructuradas del proceso, ruido de medidas e incertidumbres de modelado. El método desarrolla un conjunto de observaciones, cada uno de las cuales es sensible a un subconjunto de fallos mientras insensible a mantener fallos y entradas no conocidas. El grado de libertad extra resultante de la medida y la redundancia del modelo hacen posible construir tales observadores. La idea básica es que en un caso de árbol de fallo, los observadores sigan el proceso estrechamente y los residuos de las entradas no conocidas serian pequeñas. Si un fallo ocurre, todos los observadores los cuales están hechos insensibles al fallo por el diseño continuo para desarrollar pequeños residuos, solo reflejan las entradas no conocidas. 
por otro lado, los observadores los cuales son sensibles a los fallos se desviaran del proceso significativamente y resultaran en residuos de gran magnitud. El conjunto de observadores es así diseñado, que los residuos de esos observadores resulten en distintos patrones de residuos para cada fallo, los cuales hacen posible el aislamiento del fallo. La única forma del fallo es garantizada por el diseño donde los observadores muestran desacople de fallos completo e invarianza para perturbaciones no conocidas mientras sean independientes de los modos de fallo y la naturaleza de las perturbaciones.

Para una discusión en detalle en diseño de observadores de diagnóstico general para sistemas lineales, se puede encontrar en [30] los detalles de este tema. Una importante cuestión, dado por [30] es que en el diseño de observadores no es necesaria la aplicación de la teoría de estimación de estado, por el contrario, solo estimadores de salidas son necesarios, los cuales son generalmente realizados como filtros.

Comentarios en Evaluación Residual Muchos de los trabajos en diseño de observadores se enfocan en la generación de residuos para sistemas dinámicos con satisfactorias propiedades de desacople. Evaluación residual juega un papel importante en detección y diagnóstico de fallos subsecuente. La parte de evaluación residual considera el intercambio entre detección rápida y confiable. En muchos de los trabajos en diseño de observadores, la función de umbral simple es usada en evaluación residual. Clasificadores estadísticos también puede ser usados en evaluación residual. Una investigación en redes neuronales para evaluación residual es presentada en [57].

Relaciones de Paridad Ecuaciones de paridad son reordenadas y usualmente variantes transformadas de entradas salidas o modelos de espacio estado de la planta [53]. La esencia es chequear la paridad (consistencia) del modelo de la planta con las salidas del sensor (medidas) y las entradas conocidas de proceso. Bajo condiciones ideales de operación de estado estable, el entonces llamado residuo o el valor de las ecuaciones de paridad es cero. En situaciones reales, los residuos son diferentes de cero debido a ruidos de medidas y proceso, imprecisiones del modelo, grandes errores en sensores y actuadores y fallos en la planta. la idea de esta investigación es reordenar la estructura del modelo para obtener el mejor aislamiento del fallo. El aislamiento del fallo re- 
quiere la habilidad para generar vectores residuales los cuales son ortogonales a cada otro, para fallos diferentes. Hay alternativas atractivas debido a su habilidad para determinar, a priori, aislabilidad de diferentes fallos. Sin embargo, se debe notar que todos estos métodos son limitados a fallos que no incluyen las tendencias de parámetros grandes de proceso, y ninguno de ellos direcciona el uso de incertidumbres en fallos de parámetros multiplicativos.

Filtros de Kalman (KF) Las perturbaciones de la planta son fluctuaciones aleatorias y a menudo solo sus parámetros estadísticos son conocidos. La solución presentada por [5] y [106] al problema de diagnóstico de fallos en tales sistemas supone monitorear la predicción de errores. El objetivo es diseñar un estimador de estado con mínima estimación de error. Éste envuelve el uso de estimación de estados óptimos, es decir, el Filtro de Kalman, el cual es diseñado en la base del modelo del sistema en modo de operación normal.

Es bien conocido que los Filtros de Kalman es un algoritmo recursivo para estimación de estados y este ha encontrado amplia aplicación en Química tan bien como en otros procesos industriales. El filtro de Kalman en modelo de espacio estado es equivalente a un predictor optimo para sistemas estocásticos lineales en el modelo de entrada-salida.

Estimación de Parámetros Diagnóstico de parámetros a la deriva los cuales no son medibles directamente, requiere métodos de estimación de parámetros On-line. Modelos paramétricos precisos de los procesos son necesarios, usualmente en el dominio continuo en la forma de ecuaciones diferenciales parciales y ordinarias. Para evaluar en detalle las técnicas de estimación de parámetros consultar [109][49]. Los fallos los cuales ocurren como parámetros dependientes del tiempo a la deriva puede ser manejados a través de métodos de estimación. Estos métodos requieren la habilidad de realizar modelos dinámicos precisos del proceso y su desventaja es que son computacionalmente muy complejos para grandes procesos. La más importante cuestión en los trabajos de estimación de parámetros para diagnóstico de fallos es el de la complejidad. El modelo del proceso usado podría estar entre basado en datos de entradasalida, modelos de primer principio no-lineal o modelos de orden reducido. Si el modelo del proceso es de primer principio no lineal complejo, entonces el problema de estimación de parámetros resulta un problema de optimización no 
lineal. La solución en tiempo real a este problema es un serio cuello de botella en la aplicación de tales investigaciones.

\section{Redundancia de Hardware y Esquemas de Votado}

Los esquemas de votado son frecuentemente usadas en sistemas que poseen alto grado de redundancia de hardware [106]. Por ejemplo, considere tres medidas idénticas de sensores de la misma variable. Si una de las tres medidas difiere notablemente de las otras dos, la señal diferente es identificada como fallo, la diferencia entre las dos señales en cada par de sensores en un grupo redundante indica un fallo. Esquemas de votado son fáciles de implementar y son rápidos para identificar fallos mecánicos en instrumentos. Los sistemas de votado en aislamiento no toman ventaja de solo o incluso sensores doblemente redundantes así ignora la información potencial.

\section{Generación Residual Ampliada}

El diagnóstico residual refleja los fallos potenciales de un sistema. El paso siguiente es confirmar la presencia de un fallo e identificarlo, es decir, detectar y aislar el fallo. Para el propósito de aislamiento, es necesario generar tal diagnóstico residual para que no solo sean fallos sensibles, si no también sean fallos selectivos. Para este fin, el generador residual debe estar disponible para producir un conjunto de residuos y no solo una señal y hacer que los residuos respondan selectivamente a cada fallo potencial. El generador residual así puede servir no solo como detector de fallos sino también como clasificador de fallos.

Ha habido esfuerzos para diseñar generadores capaces de generar residuos ampliados los cuales conducen al aislamiento del fallo. Dos de tales métodos de ampliación, la investigación residual direccional [37] y la investigación residual estructural, [53][36], han atraído mucha atención debido a su capacidad de generación residual teniendo propiedades direccional o estructural, por eso facilitan el proceso de aislamiento de fallo. Los generadores residual estructurados son diseñados en tal forma que cada residuo responde a un subconjunto de fallos selectivamente. Tal diseño permite seguir fallos de forma binaria para más aislamiento. Los generadores residual direccional son capaces de generar residuos que son confinados a una dirección de fallo específico en el espacio 
residual multidimensional. Como un resultado, el aislamiento de fallo suma pasos para la determinación de una dirección predefinida al cual los residuos se hallan mas cercanos.

\section{Conclusión de los Métodos de Diagnóstico Basado en Modelos Cuantitati-} vos Se puede ver que una de las mayores ventajas de usar técnicas basadas en modelos cuantitativos es que se tiene un control sobre el comportamiento de los residuos. Sin embargo, importantes factores tales como complejidad del sistema, alta dimensionalidad, proceso no lineales y/o falta de buenos datos, frecuentemente dejan a éste mucha dificultad, incluso impráctica, para desarrollar un modelo matemático para el sistema. Esto, por supuesto, limita la utilidad de esta investigación en procesos industriales reales.

\subsubsection{Algoritmos Basados en Modelos Cualitativos}

El desarrollo de conocimiento basado en sistemas expertos fue el primer intento para capturar conocimiento y sacar conclusiones en una metodología formal. Un sistema experto es un programa de computador que imita el comportamiento cognitivo de un experto humano resolviendo problemas en un dominio particular. Éste consiste de un conocimiento base, esencialmente de un gran conjunto de reglas IF-THEN-ELSE y un motor de inferencia el cual busca a través del conocimiento base para derivar conclusiones de factores dados. También, el árbol de frases IF-THEN-ELSE crece rápidamente con la complejidad del comportamiento del sistema. El problema con esta clase de representación de conocimiento es que éste tiene cualquier entendimiento del comportamiento físico del sistema, y por lo tanto, falla en casos donde una nueva condición es encontrada y que no está definida en el conocimiento base. Por eso, esta clase de conocimiento es referido como "superficial" desde que éste no tiene una profundidad en el entendimiento fundamental del sistema.

En razonamiento simbólico, frecuentemente direcciona tres diferentes clases de razonamiento. Ellos son razonamiento de Abductivo (abductive), inductivo (inductive) y por defecto (default). Abducción es la generación de una explicación hipotética para lo que ha sido observado. Distinto de de una deducción lógica simple, nosotros podemos obtener mas que una respuesta en un razonamiento abductivo. Desde que no hay una forma general para decidir entre 
alternativas, lo mejor que se puede hacer es encontrar una hipótesis que sea la más probable. Así la abducción puede pensarse como un razonamiento donde nosotros comparemos las evidencias y las incertidumbres. Búsqueda de la causa de una anormalidad en el sistema de un proceso es así un razonamiento de abducción [102].

Aprendizaje inductivo es la clasificación de un conjunto de expresiones dentro de categorías o conceptos. Aprendizaje inductivo es realizado cuando generaliza o especializa una definición de un concepto aprendido, así que este incluye todas las expresiones que pertenecen a ese concepto y excluye estos que no son. Una clara definición de un concepto o categoría es raramente simple por la gran variedad de experiencias e incertidumbres (datos de ruido o observaciones). Por esta razón, se prefiere un esquema de aprendizaje adaptativo. Un ejemplo de esquema de aprendizaje adaptativo es el aprendizaje de manejos de fallos. Aprender el manejo de fallos es refinar un concepto de expectativas de fallos de acuerdo a las experiencias relacionadas que se tienen. El fallo de juicio heurístico en diagnóstico de fallos, consiste en detectar que una fuente de malfunción puede disparar un cambio en el conocimiento (una regla) que resulta en el juicio [102]. Experiencias con anormalidades en una planta puede ser usada para generar reglas que relacionan un conjunto de observaciones con causas específicas. Se puede refinar este conocimiento experimental con el tiempo para generalizar causas satisfactorias no cubiertas y especializadas cuando se notan excepciones.

Frecuentemente se hacen propuestas por defecto (default) en el valor de varias cantidades que son manipuladas, con la intención de permitir razones específicas para otro valor buscando anular los valores corrientes (es decir desde que la salida esté bloqueada, el flujo es ahora a cero), o de rechazar el valor por defecto si este conduce a una inconsistencia (desde que la salida del tanque esta bloqueada, allí no puede ser un decremento en el nivel del tanque). Una característica fundamental de razonamiento por defecto es que éste no es monotónico. En lógica tradicional, una vez el hecho es deducido, éste es considerado a mantener como verdadero por el resto del razonamiento. Esto quiere decir que es monotónico. Sin embargo, como una nueva evidencia surge, se necesita revisar los hechos deducidos a mantener la consistencia lógica. Consideremos nuestro argumento previo donde deducimos que el nivel del tanque no puede decrementar (desde que la salida del tanque esta bloqueada). Después 
de esta deducción, si nosotros obtenemos nueva evidencia que el tanque tiene una gran gotera, tendríamos que retractar la conclusión que el nivel del tanque no puede decrementar. Tal como un razonamiento donde las deducciones no se puede retractar es no monotónico. Razonamiento por defecto o no monotónico es una herramienta invaluable en tratar con situaciones donde toda la información no esta disponible a la vez, no se tiene mucha razón probablemente inconsistente y casos simultáneos.

La necesidad para una herramienta de razonamiento el cual pueda modelar cualitativamente un sistema, capturar la estructura casual de un sistema de una manera más profunda que el sistema experto convencional y no es aún tan rígido en naturaleza como las simulaciones numéricas, guían al desarrollo de muchas metodologías para representar el conocimiento cualitativo.

\section{Modelos Causales Basado en Grafos}

El diagnóstico es inverso de la simulación. La simulación se preocupa de la derivación del comportamiento del proceso dado los aspectos estructural y funcional. Diagnóstico por otro lado, se ocupa de la estructura de deducción del comportamiento. Esta clase de deducción necesita razonamiento acerca de la relación causa efecto en el proceso. En el estudio del razonamiento evidencial para diagnóstico, la información heurística en la forma de reglas de producción es usada. La relación de entendimiento causa-efecto del proceso están implícitas en ésta forma de razonamiento. En los trabajos de modelos basados en primer principio, se corresponde con la descripción del sistema junto con la observación hecha del malfuncionamiento del proceso. EL razonamiento aquí es para identificar los cambios funcionales con resultados en el malfuncionamiento del proceso [82][102].

Las relaciones causa-efecto o modelos puede ser representados en la forma de Di-grafos Señalizados (Signed Digraphs SDG). Un Digraph es un grafo con arcos dirigidos entres los nodos y SDG es un grafo en el cual los arcos tienen dirigido una signo positivo o negativo agregada a ellos. Los arcos dirigidos van del nodo causa al nodo efecto. Cada nodo en el SDG corresponde a la desviación del estado estable a la variable. SDGs tiene nodos los cuales representan eventos o variables y flechas las cuales representan la relación entre los nodos. Ellos son mucho más compactos que las tablas de verdad o los modelos de es- 


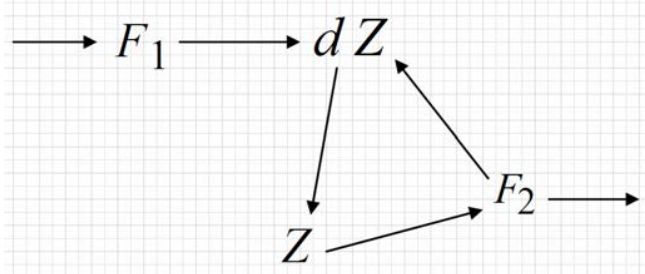

Figura 2.4: Grafo de Ejemplo de Tanque

tado finito. Para entender digraphs, considere un tanque donde $F_{1}$ es la entrada de flujo, $F_{2}$ es la salida de flujo, y $Z$ es la altura del líquido en un tanque. Las ecuaciones que representan este sistema son:

$$
F_{1}-F_{2}=\frac{d Z}{d t} \quad F_{2}=\frac{Z}{R}
$$

El correspondiente grafo se muestra en la figura 2.4 .

La figura puede ser leída como sigue: un cambio externo causa que el flujo $F_{1}$ cambie, éste causa un cambio en el nivel del líquido en el tanque ( $d Z$ y $Z$ ), éste a su vez causa el cambio de la salida de flujo $F_{2}$ y éste a su vez causa el cambio del nivel del líquido (un ciclo cerrado). El sentido de la flecha representa la dirección de cambio. En situación general, las flechas puede ser eventos independientes, es decir, la relación entre dos eventos o variables puede ser dependientes en otro evento o variables en el sistema.

SDGs suministra un muy eficiente camino de representación gráfica de modelos cualitativos. Hay tres principales clases de nodos en un típico SDG representando un proceso: (a) con solo flechas de salida. Ellos representan variables de fallo básicas o más precisas. Las cuales puede cambiar independientemente; (b) los que tienen flechas de entrada y salida, muchos frecuentemente llamados variables de proceso y (c) los que tienen solo flechas de entrada. Ellos son frecuentemente llamados variables de salida y ellos no afectan cualquier otra variable. SDGs ha sido la más amplia forma usada de conocimiento causal para diagnóstico de fallos de procesos.

\section{Árboles de Fallos}

Los árboles de fallos son usados en análisis de la fiabilidad y seguridad del sistema. El análisis de árboles de fallos fue originalmente desarrollado por Bell 
Telephone Laboratories in 1961. Los árboles de fallos es un árbol lógico que propaga eventos primarios o fallos al evento de nivel superior o de peligro. El árbol usualmente tiene capas de nodos. En cada diferente nodo hay operaciones lógicas como AND y OR que son utilizadas para la propagación. Los árboles de fallos han sido usados en una variedad de estudios de evaluación de riesgos y análisis de fiabilidad [100]. Un análisis con árboles de fallos general consiste de los siguientes cuatro pasos: (i) definición del sistema; (ii) construcción del árbol de fallo; (iii) evaluación cualitativa y (iiii) evaluación cuantitativa [59]. Antes de la construcción del árbol de fallos, el analista debe poseer un completo entendimiento del sistema.

El árbol de fallos es construido respondiendo las preguntas tales como: ¿que podría causar el evento de nivel superior? En respuesta a esta pregunta, se generan otros eventos conectados por nodos lógicos. El árbol es expandido de esta manera hasta encontrar eventos (primarios) los cuales no necesitan ser más desarrollados. Una vez el árbol de fallo es construido, el próximo paso en el análisis es la evaluación del árbol de fallos. La evaluación cualitativa está relacionada con el desarrollo de conjuntos de corte mínimo, definidos como una colección de todos los fallos primarios los cuales son necesarios y suficientes para causar la avería del sistema por el conjunto de corte mínimo en cuestión [59]. En evaluación cuantitativa, conocer acerca de la probabilidad de ocurrencia de eventos primarios es usado para calcular la probabilidad de fallo del evento de nivel superior. El término evaluación como es usado aquí es el evaluador de la probabilidad de ocurrencia del evento de nivel superior y no el evaluador de la exactitud del árbol de fallo generado.

Los árboles de fallos proporcionan un medio computacional de la lógica combinacional para analizar los fallos del sistema. La naturaleza atractiva del árbol de fallo proviene del hecho de que diferentes nodos lógicos puede ser usados (OR, AND, XOR) en vez del predominante nodo OR usado en los grafos. Esto ayuda en reducir la representación y la solución espuria del sistema en una manera concisa. El mayor problema con los árboles de fallos, es que el desarrollo es propenso a errores en diferentes etapas. El árbol de fallo construido solo es tan bueno como el modelo mental es concebido por el desarrollador. Para mantener el diagnóstico consistente de los árboles de fallos, el árbol debe representar comprensivamente la relación causal del proceso (explicando todos 
los escenarios de fallo). No hay métodos formales para verificar la precisión del árbol desarrollado.

\section{Física Cualitativa}

Física cualitativa o comúnmente sentido de razonamiento acerca del proceso físico ha sido un área de mayor interés para la comunidad de Inteligencia Artificial. EL conocimiento físico cualitativo ha sido representado en dos caminos principales. el primero es para derivar ecuaciones cualitativas de las ecuaciones diferenciales llevando a ecuaciones de confluencia. La idea básica en el desarrollo de ecuaciones de confluencia es como sigue. Considere las ecuaciones del tanque dadas en el tema de grafos. Las ecuaciones de estado estable de confluencia para este ejemplo son:

$$
\begin{aligned}
& {\left[F_{2}\right]-\left[F_{1}\right]=0} \\
& {[Z]-\left[F_{2}\right]=0}
\end{aligned}
$$

Donde $\left[F_{1}\right]$ representa la señal de la variable de desviación $F_{1}$. Esta ecuación puede ser resuelta cualitativamente para obtener el valor cualitativo de las variables. Si el valor permisible para una variable es +, entonces esto significa que la variable puede tomar solo valores altos cualitativos. es decir, éste solo puede incrementar.

Una cosa importante para notar aquí es que el comportamiento cualitativo puede ser derivado incluso si un modelo matemático preciso no puede ser desarrollado. Un modelo cualitativo no requiere información detallada (tal como valores numéricos y expresiones exactas) sobre el proceso. La información sobre los valores de operación normal de parámetros del proceso y variables es suficiente. Como un ejemplo, considere el nivel precedido del tanque como el incremento de la rata de flujo de entrada. Usando razonamiento cualitativo, se puede predecir que el nivel también incrementaría (al menos inicialmente) sin saber el valor numérico del área seccional del tanque, la salida, etc. Considerables trabajos han sido hechos en esta área de modelado cualitativo de sistemas y representación de conocimiento causal. [50] sugiere el método de 
ordenamiento causal el cual es usado para obtener la relaciones causales por la reducción conveniente del conocimiento relacional funcional sobre el proceso.

Las metas de estas metodologías son la razón de la física cualitativa, la descripción ecuacional para la descripción de comportamiento cualitativo, suministrar explicaciones de comportamiento basado en observaciones del proceso y descripción del sistema. La ventaja de estos simuladores cualitativos es su habilidad para proporcionar conclusiones parciales de conocimiento incompleto e incertidumbre frecuente del proceso. Cada una de las teorías anteriores inician de una descripción del mecanismo físico, construyendo un modelo, y entonces usan un algoritmo también para determinar todo el comportamiento del sistema, sin conocimiento preciso de los parámetros y relación funcional.

Convencionalmente, los sistemas físicos en ciencia e ingeniería son modelados usando ecuaciones diferenciales, las cuales son resueltas, entre numérica o analíticamente para proporcionar funciones que representen el comportamiento del sistema. Similarmente, los modelos cualitativos representan una abstracción del sistema físico real, y en términos de restricciones cualitativas, captura la información sobre el sistema. Estos modelos cualitativos son resueltos para obtener la descripción del comportamiento cualitativo del sistema.

Hay dos principales problemas con derivación de ecuaciones de confluencia de física cualitativa: ambigüedad y soluciones espurio. Ambigüedades puede ser resueltas completamente solo a través del uso de valores cuantitativos actuales. Soluciones espurios se refiera a la generación de soluciones no realizables físicamente por una técnica de razonamiento cualitativo. Este problema puede ser aliviado para una extensión razonable por el modelado del sistema de diferentes perspectivas [56].

\section{Jerarquía de Abstracción del Conocimiento del Proceso}

Otra forma de modelado de conocimiento es a través del desarrollo de jerarquía de abstracción basada en descomposición. La idea de descomposición es estar disponible a dibujar inferencias sobre el comportamiento del sistema total únicamente con las leyes que gobiernan el comportamiento de este subsistema. Es tal como una descomposición, la principal estructura en no funcionamiento es la central; la ley del subsistema no puede presumir el funcionamiento del sistema entero [22]. En una descripción jerárquica, se podría representar una 
descripción genérica de una clase de unidades de proceso. Las ecuaciones de gobierno describen una clase entera de unidades de proceso, que puede hacer supuestos sobre la clase como un todo, pero no puede hacer cualquier supuesto sobre el comportamiento de una unidad en particular. Como un ejemplo, para una válvula en presión regulada, el área disponible para flujo decrece como la presión incrementa. Sin embargo, esto no es verdad para todas las válvulas y la descripción en general de una clase de válvulas no puede asumir este comportamiento. Otro importante principio para descomposición del sistema es el principio de localidad: la ley para una parte específica no puede referirse a cualquier otra parte. Estructura en no funcionamiento permite comportamiento consistente entre varias unidades. El principio de localidad permite el comportamiento a ser precedido basado solo en información local. Descomposición popular de sistemas de procesos son los siguientes: (i) estructural: especifica la información conectiva de una unidad y (ii) funcional: especifica la salida de una unidad como una función de estas entradas (y posiblemente información de estado).

En jerarquía de abstracción, el sistema del proceso es descompuesto dentro de estas unidades de proceso. Esta descomposición permite una representación general de la funcionalidad de un sistema en términos de las relaciones de entrada-salida de estas unidades. Esto no es importante para el sistema de diagnóstico o el módulo de razonamiento donde la funcionalidad es expresado en términos cualitativos o cuantitativos. Se podría considerar otra forma de descripción del proceso para descomponer el sistema del proceso. Por ejemplo, se puede descomponer el sistema del proceso basado en funcionalidades abstractas. Además, no hay razón para restringirse a descripciones en el nivel de unidades. Descomposición de un sistema de proceso a subsistemas puede ser realizado en varios niveles de abstracción. Si el nivel de abstracción es sistemas de control, entonces estos subsistemas representan varios ciclos de control individual [28]. Si el nivel de abstracción es unidades, el subsistema representa unidades individuales. Esto nos trae al concepto de jerarquía de abstracción como la descripción estructural o funcional de un sistema.

Hay dos dimensiones a lo largo del cual la abstracción en diferentes niveles es posible: estructural y funcional. La jerarquía estructural representa la información conectiva del sistema y estos subsistemas. La jerarquía de abstracción funcional representa las relaciones medio-fin entre un sistema y estos subsistemas. 
La mayoría de los trabajos en diagnóstico de fallos en ingeniería química depende en el desarrollo de la descomposición funcional. Descomposición estructural está en una descomposición eficiente en sistemas donde hay una equivalencia general entre estructura y función, como por ejemplo en un circuito eléctrico. La razón para la popularidad de la descomposición funcional en ingeniería química es debido a las funcionalidades complejas de varias unidades que no puede ser expresado en términos de estructura. Así, la descomposición enfocada aquí es la descomposición funcional. Ésta jerarquía funcional describe de abajo-arriba, que varias unidades con ciertas funciones sean usadas para y como ellas sirven a propósitos de alto nivel. Ellos describen de arriba-abajo como varios propósitos son implementados a través de varias unidades con funciones específicas. La forma de proceder razonando en una descripción jerárquica depende de la tarea en mano. La información en la función apropiada de un sistema es obtenida de los niveles anteriores. Causas de funciones inapropiadas depende en los cambios en los recursos y limitaciones específicas en niveles bajos y así son explicadas de abajo-arriba (bottom-up). El diagnóstico puede ser considerado como una búsqueda de arriba-abajo de un alto nivel de abstracción donde los grupos de equipos y sistemas funcionales son considerados a un bajo nivel de abstracción donde unidades individuales y funciones de unidad son analizados [79].

\subsubsection{Métodos Basados en Historia del Proceso}

En contraste a los estudios basados en modelos donde un conocimiento a priori (cualitativo o cuantitativo) sobre el proceso era necesario, en métodos basados en historia del proceso, solo la disponibilidad de una gran cantidad de datos históricos de proceso son necesitados. Hay varios caminos en el cual estos datos puede ser transformados y presentados como un conocimiento a priori para el sistema de diagnóstico. Esto es conocido como extracción de características. Este proceso de extracción puede ser en naturaleza cualitativa o cuantitativa. Dos de los mayores métodos que extraen información histórica cualitativa son los sistemas expertos y los métodos de modelado de tendencias. Los métodos que extraen información cuantitativa puede ser ampliamente clasificados como métodos estadísticos o no estadísticos. Redes Neuronales son una importante clase de clasificadores no-estadísticos. Análisis de Componen- 
tes Principal (PCA), Mínimos Cuadrados Parciales (PLS) y Clasificadores de Patrones Estadísticos forman un mayor componente de métodos de extracción de características estadísticas.

\section{Extracción de Características Cualitativas}

A Continuación se hace revisión de los dos importantes métodos que emplean extracción de características cualitativas, ellos son sistemas expertos y estudios de modelado de tendencias.

Sistemas Expertos Extracción de características basado en reglas ha sido ampliamente usado en sistemas expertos para muchas aplicaciones. Un sistema experto es generalmente un sistema muy especializado que resuelve problemas en un estrecho dominio del experto. Los principales componentes en el desarrollo de un sistema experto incluye: Adquisición del conocimiento, elección de representación del conocimiento, codificación del conocimiento en un conocimiento base, desarrollo de los procedimientos de inferencia para razonamiento del diagnóstico y el desarrollo de las interfaces de entrada-salida. La principal ventaja en el desarrollo de sistemas expertos para resolver problemas de diagnóstico son: fácil de desarrollar, razonamiento transparente, habilidad para razonar bajo incertidumbres y la habilidad para suministrar explicaciones para el suministro de soluciones.

Hay gran cantidad de artículos que discuten aplicaciones de sistemas expertos, abordando diferentes áreas y buscando su integración con otros temas, sin embargo, hay otros artículos que plantean aplicaciones específicas de sistemas expertos para diagnóstico de fallos. Concluyendo que en todas las aplicaciones las limitaciones de un sistema experto son obvias. Desarrollo de sistemas basado en conocimiento de reglas de experto son muy específicas del sistema, su potencia de representación es realmente limitada y son difíciles de actualizar [82]. Sus ventajas son fácil desarrollo y razonamiento transparente.

Análisis de Tendencia Cualitativa (QTA) Análisis de tendencia y predicción son componentes importantes de control supervisorio y monitoreo de procesos. Modelado de tendencias puede ser usado para explicar los varios eventos importantes pasando en el proceso, haciendo diagnóstico de malfunciones y 
precediendo estados futuros. Desde una perspectiva procedimental, en orden a obtener una tendencia de señal no muy susceptible a variaciones momentáneas debido al ruido, algunas clases de filtros necesitan ser empleados. Por ejemplo, representaciones de series de tiempo supone, a priori, cierto comportamiento como ellos están identificando usando un comportamiento del proceso conocido. Alternativamente, un camino simple usa un filtro (tal como un filtro autoregresivo) con elección a priori de los coeficientes del filtro (especificando el grado requerido de lizado). Ambos tipos de filtros parten del hecho que ellos no puede distinguir bien entre un transitorio y la inestabilidad verdadera [53]. Las características esenciales cualitativas puede ser distorsionadas por estos filtros. Para evitar este problema requiere que la tendencia sea vista de diferentes escalas de tiempo o diferentes niveles de abstracción. Abstracción cualitativa permite una representación compacta de la tendencia por la representación solo de los eventos significativos. Para tareas tales como diagnóstico, representación de tendencia cualitativa frecuentemente suministra información valiosa que facilita el razonamiento sobre el comportamiento del proceso. En la mayoría de los casos los malfuncionamientos del proceso salen a distintas tendencias en el monitoreo de los sensores. Estas distintas tendencias puede ser adecuadamente utilizadas en identificar el comportamiento anormal en el proceso. Así, una clasificación adecuada y análisis de la tendencia del proceso puede detectar el fallo tempranamente y guiar a un rápido control. También, representación de tendencia cualitativa puede pavimentar el camino para compresión de datos eficientes.

\section{Extracción de Características Cuantitativas}

Los estudios cuantitativos esencialmente formulan el problema del diagnóstico resolviéndolo como un problema de reconocimiento de patrones. La meta del reconocimiento de patrones es la clasificación de puntos de datos, en general, clases predeterminadas. Métodos estadísticos usan conocimiento de distribución de clases a priori para realizar clasificación. Un ejemplo es un Clasificador Bayesiano el cual usa las funciones de densidad de las respectivas clases. Estudios como Análisis de Componente Principal (PCA), por otro lado, extraen información sobre mayores tendencias en los datos usando un pequeño número de factores relevantes. Redes neuronales asume una forma funcional para 
las reglas de decisión así parametrizando el clasificador. A continuación se comentan estas técnicas usadas en diagnóstico.

Extracción de Características Estadísticas de Datos del Proceso. En operaciones de proceso real, se está enfrentado con el problema de tratar con sistemas sometidos a perturbaciones randómicas. En contraste a sistemas determinísticos, el estado futuro de sistemas estocásticos no es completamente determinado por estados pasado y presente y acciones de control futuras. Las medidas son consideradas series de tiempo estadísticos a una sola realización de un proceso estocástico subyacente. Desde que el sistema esta bajo influencias randómicas, éste es razonable o algunas veces necesario a formular el sistema en una ubicación probabilista. Cuando el proceso esta bajo control, las observaciones tienen distribuciones de probabilidad correspondiendo al modo normal de operación. La distribución de comportamiento cambia cuando el proceso esta fuera de control. En general, distribuciones de probabilidad son caracterizadas por sus parámetros cuando un estudio paramétrico es usado. Por ejemplo, si la distribución subyacente de una variable de monitoreo es normal, entonces los parámetros de interés son los valores de la media y la desviación estándar. Bajo condiciones de fallo, entre la media y la desviación estándar puede desviarse de sus valores nominales. Un cambio compuesto puede ocurrir también. Acordemente, en diagnóstico de fallos puede ser declarado como el problema de detectar cambios en los parámetros de un sistema estocástico estático o dinámico. [6] presenta el diseño de algoritmos de detección de cambios on-line y un análisis de su comportamiento bajo un espacio de trabajo unido. Los estudios Bayesiano y no-Bayesianos son discutidos en el trabajo citado anteriormente.

La creciente demanda para calidad y procesos confiables han guiado a un extensivo uso de cartas de control de procesos estadísticos (SPC). Aunque los conceptos básicos detrás de tales métodos SPC son todavía validos, los métodos gráficos usados para implementarse en ellos no puede acomodarse al progreso en tecnología de adquisición de datos. En adición, en el evento en que los parámetros monitoreados no son independientes, el uso de cartas control univariable puede ser engañoso y puede causar confusión debido al hecho de que el método univariable no puede manejar correlación. 
Técnicas Multivariable estadísticas son herramientas poderosas capaces de comprimir datos y reducir dimensionalidad, así que la información esencial es conservada y fácil para analizar en el enorme conjunto de datos original; y está también disponible para manejar ruido y correlación extrayendo efectivamente información verdadera.

- Análisis Principal de Componentes PCA/ Mínimos Cuadrados ParCIAL (LES): PCA fue inicialmente propuesto por Pearson (1901) y después desarrollado por Hotelling (1947), es una técnica multivariable estándar y ha sido incluida en muchos libros [107] y artículos [51]. La principal función de técnicas estadísticas multivariable es para transformar un número relacionado de variables de proceso a un conjunto pequeño de variables no correlacionadas.

Entre los mediados de los años 60 y los primeros de los 80's, el método PLS fue desarrollado por Wold y Coworkers, similar a PCA, conceptualmente, PLS es útil en reducir las dimensiones de variables de procesos y variables de calidad de producto a ser analizado. Teóricamente, PCA es basado en una descomposición ortogonal de la matriz de covarianza de las variables de proceso a lo largo de direcciones que explican la máxima variación de los datos. El principal propósito de usar PCA es para encontrar factores que tengan una muy baja dimensión que el conjunto de datos originales los cuales puede apropiadamente describir la mejor tendencia en el conjunto de datos original. La mayor limitación de monitoreo basado en PCA es que el modelo PCA es invariante en el tiempo, mientras muchos de los procesos reales son variantes en el tiempo. Así el modelo PCA deber recursivamente actualizado.

Contrario a los trabajos de métodos basados en modelos, métodos estadísticos multivariables no necesitan modelo del sistema explicito. Ellos son capaces de manejar alta dimensionalidad y variables de proceso correlacionada y ellos son herramientas poderosas de revelado de la presencia de una anormalidad. Sin embargo, ellas no poseen propiedades de tendencia para diagnóstico, los cuales hacen difícil el aislamiento del fallo. Un poco de técnicas han sido propuestas; sin embargo, no hay solución completa a este problema aún. Allí han habido algunos esfuerzos para combinar las ideas de los trabajos basado en modelos a este método estadístico multivariable [?]. 
- Clasificadores Estadísticos: Diagnóstico de Fallos es un problema de clasificación y así puede ser proyectado en un espacio de trabajo de reconocimiento de patrones estadístico clásico. Los clasificadores Bayesianos es un clasificador optimo (Clasificador de error mínimo incluso cuando las clases se traslapan) cuando las clases son distribuciones Gaussianas y la información de distribución esta disponible. Note que la distancia medida en la forma de la escala es usada aquí. Note también que un clasificador basado en distancia el cual escala esta distancia en este modo, será el mismo como las reglas de decisión de Bayes para distribuciones Gaussianas.

Una distribución guassiana aparece como una elipsoide cuando se traza en el espacio de variables de entrada. La inversa de la covarianza sirve para transformar la elipse en una esfera de radio uno. Así, la distancia métrica sirve para transformar el problema (distribución elipsoidal) a una forma simétrica esférica antes que la clasificación sea hecha. Hay otra clase de clasificación de distribución libre que reduce la necesidad para un numero grande de muestras para hacer supuestos más precisos sobre la forma funcional de la función discriminante. En particular, se puede especificar la forma funcional para el clasificador, sacando un conjunto finito de parámetros a ser estimados. La más común elección son clasificadores cuadráticos.

El diagnóstico de Fallos puede ser considerado como un problema de combinación, con el tiempo, la estimación instantánea del clasificador usando el conocimiento sobre las propiedades estadísticas del modo de fallo del sistema [80].

Redes Neuronales (NN) Considerable interés ha sido mostrado en la literatura en las aplicaciones de redes neuronales para el problema de diagnóstico de fallos. Redes neuronales ha sido propuesta para problemas de clasificación y función de aproximación. En general, las redes neuronales que han sido usadas para diagnóstico de fallos puede ser clasificada a lo largo de dos dimensiones: (i) la arquitectura de la red tal como un sigmoide (sigmoidal), radial base, entre otros; (ii) estrategia de aprendizaje tal como aprendizaje supervisado y no supervisado. 
En estrategias de aprendizaje supervisado, para elegir una topología específica para la red neuronal, la red es parametrizada en el sentido que el problema a manejar es reducido a la estimación de los pesos de conexión. Los pesos de conexión son aprendidos explícitamente utilizando la diferencia entre el valor actual y el deseado a guiar la búsqueda. Esto hace que las redes neuronales supervisadas sean una buena elección para clasificación de fallos, ya que, las redes son capaces de generar y clasificar arbitrariamente regiones en el espacio [19]. Al final del espectro están las arquitecturas de redes neuronales que utilizan técnicas de estimación no supervisada. Estas redes son conocidas popularmente como redes neuronales de organización propia (self-organization) como la estructura es determinada adaptativamente basada en la entrada de la red. Un ejemplo puede ser la red ART2 [13].

\subsection{Trabajos Previos en Diagnóstico de Fallos de Sistemas de Eventos Discretos}

Las dos herramientas de modelado más utilizadas para diagnóstico de fallos de Sistemas de Eventos Discretos (SED's) en los últimos años son las Máquinas de Estado Finitas (MEF's) y las RdP, teniendo mayores aplicaciones las RdP por sus grandes fortalezas gráficas y matemáticas. Por lo anterior, se busca citar los trabajos realizados recientemente donde se usan estas herramientas por separado o unidas. La base de los trabajos que se citan a continuación está realizado en MEF's. Este trabajo fue realizado por [86], el trabajo está distribuido en dos áreas: la construcción del modelo y la construcción del diagnosticador con su respectiva diagnosticabilidad. Por ser el trabajo principal se da una explicación detallada, de la cual se desprenden los demás trabajos.

En la construcción del modelo del proceso se parte de realizar el modelo de eventos discretos de cada uno de los componentes del sistema, en éstos se debe tener en cuenta el comportamiento normal y de fallo, después, se realiza el modelo de composición sincrónica que integra en un solo modelo a todos los eventos y estados del sistema, está compuesto por eventos observables y no observables. Los eventos de fallo están enmarcados como no observables. Para poder dar paso a la construcción del diagnosticador, primero se realiza una simplificación del modelo de composición, identificando eventos observables, no observables y eventos nuevos en el sistema, los eventos nuevos surgen de la in- 
terpretación de las lecturas sensoriales. Así el modelo queda reducido sólo con eventos de fallo, eventos de supervisor y eventos nuevos. Este modelo aunque reducido tiene una gran limitación y es que el número de estados del modelo de composición esta dado por la productoria de los eventos de los componentes del sistema, es decir, para el ejemplo de HVAC que se cita en [88], por tres elementos que se tienen cada uno con cuatro estados, el modelo de composición queda con 64 estados, llevando a que si el modelo aumenta en elementos, esta construcción es imposible de realizar.

El paso siguiente en el trabajo fue el de construir el diagnosticador. El diagnosticador es una MEF's que realiza una observación on-line del proceso, la cual esta basada solo en eventos observables del modelo de composición. Éste es un procedimiento sistemático que describe: definición de las etiquetas de fallo, definición de las funciones de: propagación de etiqueta, corrección de etiqueta y función de Rango. También define propiedades a los estados del diagnosticador de cierto, incierto, ambiguo y ciclo indeterminado. Éstas características son definidas para que el diagnosticador cumpla las funciones de observador. Así por último define la diagnosticabilidad: un sistema es diagnosticable cuando el diagnosticador no contiene ciclos indeterminados. Su principal desventaja es que el sistema en pocas circunstancias cumple la condición para la diagnosticabilidad.

En general, esta metodología presenta varios inconvenientes: es rígida, es decir, los fallos tienen que suceder en determinada forma y en un proceso real nunca se puede definir como suceden los fallos. Solo permite el diagnóstico de un fallo, para fallos múltiples, simultáneos y dependientes no se puede aplicar; y por último la desventaja más grande es la explosión combinacional, esto hace que sólo pueda ser aplicada a procesos pequeños; cuando la complejidad del proceso aumenta, es imposible aplicar esta metodología.

Basado en el trabajo mencionado anteriormente, buscando dar mejora a los problemas planteados se desarrollo el trabajo de [33]. Pretendiendo reducir el problema de la explosión combinacional, esta técnica plantea hacer una descomposición modular del diagnosticador, es decir, que para poder aplicar a procesos más grandes, se podía distribuir el sistema en módulos y así se podría hacer un mejor diagnóstico. Aunque se redujo en gran parte el problema de la explosión combinacional, se sigue presentando los inconvenientes dados en el trabajo de [88]. En la investigación elaborada por [18], como aporte tiene que el diagnóstico de fallos es enfocado en el àrea de fallos intermitentes, se aporta 
un nuevo estado donde permite tener una variable de tiempo que mide la duración del fallo intermitente y una variable de conteo, que sirve para que aporte al supervisor una predicción en cuando está próximo a suceder un fallo, por ser un trabajo elaborado con MEF's se siguen dando las dificultades presentadas anteriormente.

Otros aportes en la línea de SED's están los desarrollados por [11][15][101], estos investigadores tienen en sus desarrollos el modelo hecho con RdP y el diagnosticador elaborado con MEF's. A continuación se describe algunas características de los autores mencionados anteriormente. [101] realiza el modelo del sistema con RdP, el modelo dado en el artículo no representa ningún proceso real y su tamaño es muy reducido, manifiesta que todas las transiciones son no observables. En cuanto al diagnosticador plantea el mismo proceso de construcción de [87] con la variación que en la función de propagación de etiqueta incluye el marcado alcanzable de la RdP. Mantiene el mismo concepto de diagnosticabilidad y por lo tanto sus desventajas son mantenidas casi en su totalidad. Para el trabajo de [15] es idéntico con la pequeña variación de que las transiciones dejan de ser todas no observables y alguna pasan a ser observables, esto ocasiona un incremento en la diagnosticabilidad ya que puede ser estimado el marcado de una manera más precisa. Hace la variación en las funciones de propagación de etiquetas y de rango. El diagnosticador de igual forma se mantiene idéntico con las mismas desventajas y el modelo es simple y sin representar ningún proceso real. Con esta misma orientación esta la investigación de [11], el modelo realizado en RdP no representa un proceso real y en su comportamiento normal, las transiciones que hay son observables y no observables, para decir este supuesto no argumenta ninguna justificación. En la construcción del diagnosticador hay un mejor aprovechamiento de la potencia matemática de las RdP, pero finalmente el diagnóstico lo realiza a través del árbol de cubrimiento (coverability). Estos desarrollos aunque dan nuevas aplicaciones no mejoran los problemas dados por [86] y por el contrario, se pierden algunas características buenas del diagnosticador que se tenían como las funciones de supervisor informando el estado actual del proceso.

También, se presenta los trabajos de [78][84] que en concepto del autor de este documento, son los que presentan una mejor herramienta de diagnóstico en RdP. Su modelo es realizado con RdP Interpretadas, describe el funcionamiento de un proceso real, le dan aprovechamiento a la potencia matemática de 
las RdP. Presentan un algoritmo para la construcción del modelo y su diagnosticador es sistemático, ya que, matemáticamente compara y evalúa el marcado normal con el de fallo, así identifica el fallo y a través de un algoritmo encuentra el lugar que ha fallado, aunque es bueno, tiene dificultades que sólo identifica un fallo y su modelo de RdP entra en un lugar sumidero bloqueándose.

Por último está la investigación de [35], en él realiza diagnóstico de fallos utilizando RdP con lugares limitados, utiliza el mismo ejemplo de HVAC de [88], en este trabajo modela los componentes sin hacer composición sincrónica, hace un algoritmo de diagnóstico distribuido donde identifica los fallos de manera independiente. El diagnóstico es simulado inicialmente con Matlab y después es integrado con la herramienta Graphviz de AT\&T. Esta técnica es compleja de implementar y poco posible de aplicar a procesos industriales con mediano grado de complejidad.

\subsection{Trabajos Previos en Diagnóstico de fallos de Sistemas Híbridos}

Un concepto que se confunde a menudo es el de Híbrido, ya que, es utilizado por muchos investigadores en diferentes áreas de la industria, ésto es debido a que su definición dice que híbrido es "todo lo que es producto de elementos de distinta naturaleza". En este trabajo previo a conocer las investigaciones realizadas con diagnóstico de fallos de sistemas híbridos, es una tarea de selección de información que sólo contenga el concepto de híbrido en el área que se definió anteriormente, que son sistemas que involucren partes continuas y discretas de un proceso.

En diagnóstico de fallos de sistemas híbridos, se puede clasificar las investigaciones de acuerdo a las técnicas que utilizan en su implementación, hay herramientas con muchos avances ya realizados, como las que usan: Autómatas Híbridos, Redes de Petri Híbridas, entre otras; y otras que no tienen definida una técnica específica y por el contrario combinan diferentes técnicas. por lo tanto, es de suma importancia conocer de los diferentes trabajos realizados en estas dos áreas de clasificación.

Uno de los más importantes puntos tratados en documentos de FDI es que ningún método solo es adecuado para manejar todos los requerimientos de un sistema de diagnóstico. Aunque todos los métodos tienen restricciones, en el sentido de que se ha mostrado que ellos son tan buenos como la calidad de la 
información les sea suministrada, se mostró que algunos métodos puede funcionar mejor con el conocimiento disponible de otros. Se sabe que algunos de éstos métodos puede complementarse con otros resultando en mejores sistemas de diagnóstico. Integrando estas características complementarias es un camino para desarrollar métodos híbridos que podrían superar las limitaciones de estrategias de solución individual. Así, investigaciones híbridas donde diferentes métodos trabajan en conjunción se usan para resolver partes del problema.

Como un ejemplo, la explicación de fallo a través de una cadena causal es mejor hecha a través del uso de grafos (diagraphs), mientras que, el aislamiento de fallo puede ser muy difícil usando grafos debido a la ambigüedad cualitativa y el método basado en modelos analíticos puede ser superior. Así, los métodos híbridos pueden suministrar en general una poderosa plataforma de solución de problemas [103].

En el trabajo citado por [64], se diagnostica sistemas dinámicos complejos, donde se examinan sistemas continuos con controladores supervisorios, que experimentan abruptamente fallos parciales o definitivos en los dispositivos del sistema. Asumen el problema de diagnóstico como un problema de selección. Se explotan las técnicas de razonamiento cualitativo que induce a un pequeño conjunto de modelos, el cual es refinado usando estimación de parámetros. Por otro lado, [113] realiza una de las aplicaciones más interesantes, desarrolladas hasta el momento en diagnóstico de sistemas híbridos; todo el trabajo es realizado en el sistema alimentador de papel de una impresora Xerox. Sus aportes son excelentes porque hace una integración híbrida de técnicas continuas y discretas de FD como: Autómatas Híbridos, Redes de Petri Temporizadas, Árboles de Fallo y Técnicas de procesamiento de señales, que en conjunto solucionan un problema de diagnóstico. [27] trabaja FD en sistemas híbridos combinando diagnóstico basado en modelos junto a procesamiento de señales. Como se puede percibir estas investigaciones aunque no usan una técnica específica, si no la combinación de varias, mantienen el FD en sistemas híbridos.

Aunque los sistemas híbridos han existido siempre, los procesos que contienen manejo de variables continuas, han venido siendo discretizadas para realizar un diagnóstico desde el punto de vista de SED's. Esta área se inicia fundamentalmente utilizando como herramienta los Autómatas Finitos, con ellos se ha podido realizar varias investigaciones que han sido citadas a lo largo del documento, por lo tanto, en el trabajo de [41] se crea la teoría del Autómata Híbrido, 


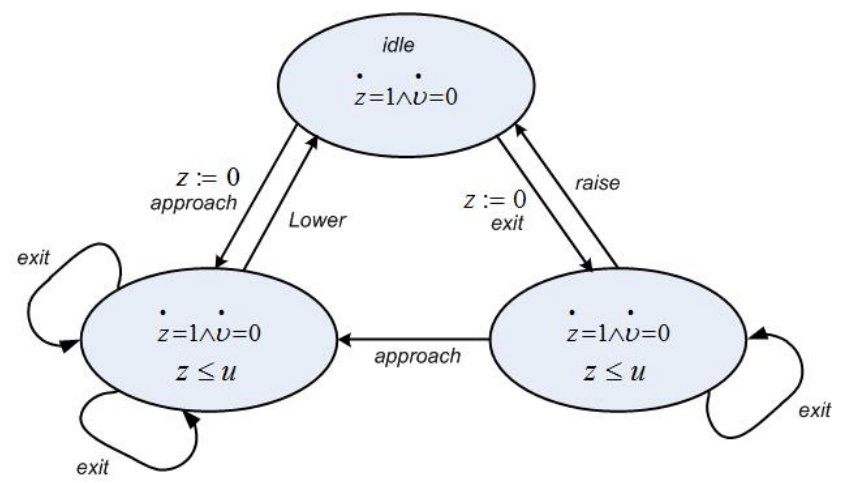

Figura 2.5: Autómata de Controlador Híbrido

donde con sus fundamentos implementa la dinámica continua junto a la discreta, ver figura 2.5. Éste es el inicio para que se den trabajos relevantes en el uso de autómatas híbridos. Investigaciones que usan esta herramienta está la de [29]; en ella discute la noción de diagnosticabilidad de sistemas híbridos en el espacio de trabajo de autómatas híbridos de entrada-salida, los estados reflejan el estado normal y de fallo de los componentes del sistema. Otros trabajos que orientan su desarrollo partiendo de SED's a sistemas híbridos son los de [14] y [58]. Éstos basan sus trabajos en [41] y realizan análisis discreto y control de sistemas híbridos.

Los sistemas de manufactura automáticos, los cuales son caracterizados por la dinámica, concurrencia y sincronización, son un sistema Híbrido que no puede ser modelado trivialmente, por ello, se han visto obligados los investigadores a buscar alternativas de modelado y diagnóstico. La alternativa que mayor cubrimiento da a las exigencias de los sistemas híbridos son las redes de petri. Para poder dar el salto de SED's a sistemas híbridos, [20] primero emite los conceptos de redes de petri continuas, trabajando el modelado de sistemas continuos, para posteriormente avanzar a definir las redes de petri híbridas, donde se combinan estados y transiciones que puede surgir de cualquiera de las dos partes de un proceso híbrido, continuo o discreto. Esta herramienta sirve de plataforma para dar un inicio certero en el modelado y diagnóstico de fallos de sistemas híbridos. Basados en ésta herramienta se producen las investigaciones hechas por [47] [110] y [81]. Todos implementan modelado y FD basados en redes de petri híbridas. El trabajo de [47] presenta una aplicación real de un sistema de manufactura híbrida, la cual es implementada en la herramienta de software VI- 


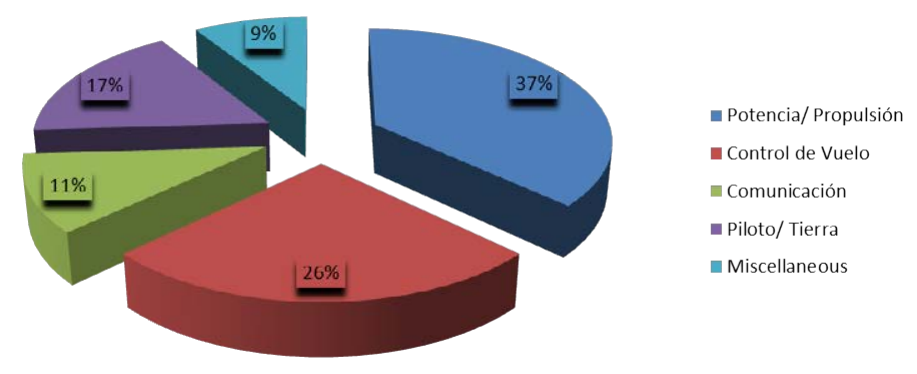

Figura 2.6: Estadística de Fallos en Aeronaves

SOBJNET y abre un buen inicio en la aplicación de las redes de petri híbridas en la industria.

\subsection{Trabajos Previos en Diagnóstico de Fallos de UAV's}

El objetivo principal de ésta investigación es poder crear una herramienta de FD que sea aplicada a los Robots Aéreos, mas concretamente a las aeronaves no tripuladas o UAV's. Para ello, como antesala a mostrar todo el proceso de construcción sistemático de la herramienta de FD, se hace necesario profundizar en los trabajos encontrados acerca del FD específico en UAS.

Los UAS vienen siendo utilizados décadas atrás, teniendo gran impulso en el campo militar, por lo tanto, es allí donde únicamente existen fuentes de información acerca de la confiabilidad en el empleo de UAS y sus implicaciones para con la población civil. Entre otras muchas formas, los accidentes de los UAS se pueden clasificar según el tipo de costo que suponga el accidente, desde el costo por daños materiales y humanos causados. Otra posible clasificación sería la basada en el motivo del fallo, como por ejemplo: Potencia/Propulsión, Control de Vuelo, Comunicación, Control de Tierra/factores humanos, Misceláneo (Otros). Según datos tomados de los estudios de confiabilidad de [68], los fallos están distribuidos en la gráfica 2.6 .

En el área de diagnóstico de fallos de UAS, según [40] los fallos que pueden ocurrir en una aeronave no tripulada, pueden clasificarse según tres dominios fundamentales: el domino del diseño, el dominio de la tripulación de vuelo y el dominio operacional. En estos dominios se puede evidenciar peligros tales 
como: impactos en tierra con daños colaterales a personas y propiedades, y colisión en el aire con aeronaves tripuladas. Aunque en primera instancia pareciera que los problemas son los mismos de una aeronave tripulada, se debe prestar gran atención a los riesgos que implica la separación de la cabina de la misma aeronave.

Las Técnicas de Diagnóstico de fallos (FD) han sido ampliamente usadas en procesos de la industria para detectar fallos en actuadores, sensores o componentes y generar información crucial para alcanzar un diseño de sistema de control tolerante a fallos (FTC) satisfactorio. Con Técnicas FD, la estrategia de control o la planeación de la misión después de la detección de un fallo pueden ser cambiadas. Generalmente hablando, FD contiene tres pasos: detección de fallos, Aislamiento y Estimación del Fallo. Detección de fallos es para decidir si un fallo o no ha ocurrido, aislamiento del fallo es para determinar la localización del fallo y estimación del fallo es para determinar la clase de fallo y su severidad. Técnicas de FTC y FD son creados para incrementar la fiabilidad y la seguridad en el sistema.

Desde el punto de vista del diagnóstico de fallos, la mayoría de las investigaciones [63][25][39] están enfocadas en evaluar los fallos en el hardware ubicado en la aeronave [10][112] [8] (sensores y actuadores), pero se debe tener en cuenta los fallos respecto de los enlaces de comunicación y también los de la estación de control. Por otro lado, hay investigaciones que sus esfuerzos se enfocan en identificar fallos y buscar una reconfiguración en el sistema de control [25][17][7][24] para llevar la aeronave a un estado de funcionamiento normal o en el peor de los casos abortar la misión. La mayoría de las técnicas utilizadas están basadas en estimación de parámetros [85], redes neuronales [74] y en algunos casos aplican redundancia [8].

A continuación se va a realizar una revisión de las Investigaciones de FD basadas en Modelo Analítico, en procesamiento de señales, y basadas en conocimiento.

\subsubsection{Métodos Basados en Modelo Analítico.}

Los estudios de FD basado en modelo Analítico consideran el modelo matemático para realizar el FD en tiempo real. Obtener el modelo es una tarea muy compleja y difícil. Muchos grupos de investigación, están construyendo sus 
propias plataformas de UAV para sus propósitos de investigación y un número de métodos de identificación de sistemas han sido propuestos para derivar modelos lineales o no lineales para condiciones de vuelo específicos [12]. Generalmente hay tres tipos de modelos: Modelo Tiempo Invariante Lineal (Linear Time-Invariant LTI), Modelo de variación de parámetro Lineal (Linear Parameter Varying LPV) y modelo no lineal.

Investigaciones basadas en modelos principalmente incluyen varios tipos de observadores y Filtros de Kalman (KF) para estimación de estados o parámetros. [111] asume que $f(x)=0$ después de la ocurrencia de un fallo para simplificar el diseño del observador y usar éste para la estimación del fallo de un HC no tripulado en el plano vertical. [45][44] usaron un modelo de salidas/entradas y observador de Luenberger para estimación de fallos de actuadores y sensores. Esto puede ser visto desde el resultado experimental, como un fallo de salida constante de sensor o un fallo de sensor fuera de orden, que son fácilmente detectados por el sistema FD con un corto tiempo de retardo. Sin embargo, un fallo de sensor con error aditivo o multiplicativo es detectado dependiendo de la magnitud del fallo. Si el rango entre la magnitud del fallo y el nivel de ruido son muy pequeños, ellos no pueden ser detectados. [104] ha diseñado observadores de aislamiento de fallos para sistemas lineales cuadrados y no cuadrados, y suministra un diseño que garantiza estabilidad de los observadores y minimiza la influencia de las perturbaciones en los residuos al mismo tiempo. [67] ha desarrollado un observador de entrada no conocida (UIO) con sistema LPV para identificación de fallos de un $\mathrm{HC}$ no tripulado de dos grados de libertad, [62] diseñó un UIO para seguir parámetros de fallos de actuador y desacoplar el efecto de fallos y entradas no conocidas.

Filtros de Kalman estándar (KF), Filtros de Kalman Extendido (KFE) y Filtros de Kalman Unscented (UKF) son utilizados. El KF en modelo de espacio estado es equivalente a un predictor óptimo para un sistema estocástico lineal. UKF aproxima la distribución del estado con un conjunto finito de puntos. Desde que los modelos no lineales son usados sin linealización, es más simple implementar y consume menos tiempo para una aplicación de tiempo real comparada con KFE. [73][70][75][72] proponen estimación adjunta de parámetros y estados basado en UKF de raíces cuadradas y UKF adaptativo basado en KF con un modelo lineal completo de un helicóptero no tripulado (UHC). EI UKF adaptativo basado en KF es compuesto de dos filtros maestro esclavo. Comparado a 
UKF el adaptativo es más simple y es un método de estimación altamente efectivo. [43][46] obtuvo el modelo de UHC desde datos experimentales de entrada salida con el método de identificación de filtros de Kalman/Observador (Observer/Kalman Filter Identification (OKID)) y presentó un sistema para detección de fallos de sensores de HC basado en el método OKID. La principal ventaja de este método propuesto es que no hay necesidad de estimar ni las matrices del sistema ni las matrices de covarianza de ruido de medición y proceso, debido a que toda la información es extraída de los datos entrada salida experimental.

En una forma diferente, [108] propuso un método de filtro de configuraciónmiembro extendido adaptativo (Adaptive Extended Set-Member Filter (AESMF)) para diagnóstico de fallos de sensores. Filtro Set-Member es un investigación para procesar datos de ruido limitado pero no conocido, y el resultado final es un conjunto el cual incluye el valor verdadero. Bajo circunstancias normales, el centro del conjunto puede ser reconocido como una estimación de datos de ruido. Comparando con métodos KF los cuales son basados en características de ruido estocástico, filtro set-member requiere datos de ruido, siendo limitados y conocidos pero no requiere propiedades estadísticas de datos de ruido, como media y desviación estándar. Entonces, los métodos set-Member tiene la ventaja de amplia adaptación y robustez fuerte.

\subsubsection{Métodos basados en Procesamiento de Señales.}

Investigaciones basadas en procesamiento de señales pueden ser usadas para sistemas lineales y no lineales, basados directamente en datos de señal y no requiere de un modelo analítico preciso. Métodos basados en procesamiento de señal son construidos en la base de un análisis en profundidad en el mecanismo fallido, para determinar características de señal, las cuales pueden mayoritariamente representar fallos. Algunos investigadores usan tecnología de la transformada de Wavelet. [71] propuso una novedosa investigación basada en wavelet para detectar fallos abruptos de sensores en un sistema de UHC. [61] definió una efectiva metodología de detección y localización de fallos de engranajes usando sensores de emisión acústica para dividir el par de la caja de cambios analizando el tiempo de llegada de las explosiones de emisiones acústicas para determinar la localización del fallo del engranaje. [105] presentó una validación de la técnica de redundancia analítica basada en Wavelet en una plata- 
forma de UHC de tres grados de libertad. Además de la técnica Wavelet, [55] detectó los fallos a través de medir el rango de cambio de datos con respecto al tiempo. [89] propuso una metodología para predecir el fallo del cojinete del elemento rodante del HC. Esto incluye una serie de pasos de procesamiento priores, incluyendo extracción de características, selección de características, y evaluación de la salud. Los autores esbozan las ventajas y desventajas de métodos diferentes en cada paso. En el análisis de tiempo-frecuencia, algunos métodos han sido propuestos para fallos particular tales como [38].

Muchos de los artículos sobre métodos basados en procesamiento de señal apuntan al sistema de transmisión del HC, como rodamientos y engranajes, basados en análisis de señal de vibraciones. Monitoreo de Vibraciones es ampliamente usada para observar la condición de un proceso o equipo. Normalmente, es difícil recolectar señales de vibración de la fuente directamente, debido al diseño y construcción de la maquinaria. Por lo tanto, sensores de vibración los cuales son típicamente acelerómetros tienen que recolectar indirectamente señales de vibración. Eso significa que la transmisión de la señal de vibración desde la fuente al sensor es compleja y fácil de ser perturbada. Por lo tanto, la tarea de investigaciones basada en procesamiento de señal no es solo para reconocer la diferencia entre una condición de fallo o normal, si no también para separar la información útil de la señal medida a la original. Algunos métodos para extraer señales han sido ampliamente usados como análisis espectral y análisis estadístico de frecuencia. Acorde a éstas características, especificar un fallo o avería puede ser detectado. Además, con adición de clasificador, varios tipos de fallos/averías pueden ser aislados.

\subsubsection{Métodos Basado en Conocimiento.}

Un amplio rango de información en relación con objetivos de diagnóstico es requerido por las investigaciones basadas en conocimiento. En particular, éstas investigaciones pueden hacer completo uso de conocimiento de expertos en el campo y evitar una dependencia en modelo matemático preciso. Entre éstas investigaciones [76] presentó un esquema de NN adaptativa para diagnóstico de fallos de sensor UHC. La investigación de umbral adaptativo elimina la necesidad de umbrales cambiando con la condición de variación de vuelo. 


\section{Capítulo 3}

\section{Fundamentos de Redes de Petri}

Las Redes de Petri (RdP) son una herramienta de modelado gráfico y matemático aplicada a muchos sistemas. Es una herramienta con alta proyección en el campo de la automática, en la cual se puede estudiar y describir información de sistemas de procesamiento que son caracterizados por ser concurrentes, paralelos, asíncronos, distribuidos, no determinísticos y/o estocásticos. Como herramienta gráfica las RdP pueden ser usadas como una ayuda de comunicación visual similar a las cartas de flujo, diagramas de bloques y redes. En adición las marcas son usadas en estas redes para simular la dinámica y actividades de concurrencia de sistemas. Como herramienta matemática es posible hacer ecuaciones de estado, ecuaciones algebraicas y otros modelos que gobiernan el comportamiento de sistemas.

En este capítulo del documento se exponen conceptos básicos de RdP, los cuales son necesarias para poder abarcar los temas posteriores. A continuación se presentan los temas de RdP con sus características más importantes, las cuales son usadas para la construcción del modelo y diagnosticador de la presente investigación. Para buscar un entendimiento más profundo del tema de RdP, se sugiere consultar las referencias de [90][20][66].

\subsection{Conceptos Básicos}

Una Red de Petri (RdP) tiene dos tipos de nodos, llamados lugares y transiciones. Un lugar $(P)$ es representado por un círculo y una transición $(T)$ por una barra, ver figura 3.1. Los lugares y transiciones son conectados por arcos. El número de lugares y transiciones son finitos y no cero. Un arco es directamente 


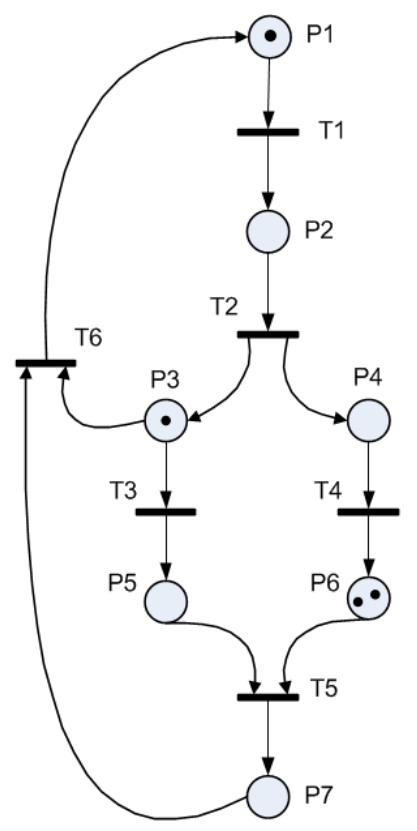

Figura 3.1: Red de Petri

conectado de un lugar a una transición o de una transición a un lugar. En otras palabras una RdP es un grafo bipartito, es decir, que los lugares y transiciones alternan en un camino hecho de arcos consecutivos. Por lo cual, es obligatorio que cada arco tenga un nodo en cada una de sus terminaciones, haciendo referencia al hecho de identificar transiciones de entrada y transiciones de salida, asociadas a un lugar de entrada o salida en particular de la red.

Marcado. Cada lugar contiene un entero (positivo o cero) de marcas. El número de marcas contenidas en un lugar $P_{i}$ es llamado $m\left(P_{i}\right) \circ m_{i}$. La red marcada, $m$, es definida por el vector de ese marcado, es decir, $m=\left(m_{1}, m_{2}, \ldots, m_{n}\right)$. El marcado en un cierto momento define el estado de la RdP, o más precisamente el estado del sistema descrito por la RdP. La evolución del estado por lo tanto corresponde a la evolución del marcado, causada por el disparo de transiciones.

Disparo de una Transición. Una transición puede solo ser disparada si cada uno de los lugares de entrada de esta transición contiene al menos una marca. La transición entonces se dice que es disparable o está habilitada. El disparo de una transición $T_{j}$ consiste en quitar una marca de cada uno de los lugares de entrada de la transición $T_{j}$ y adicionar una marca a cada uno de los lugares de salida de la transición $T_{j}$. Cuando una transición es habilitada, ésta 
no implica que será inmediatamente disparada, ésta solo mantiene esa posibilidad. El disparo de una transición es indivisible, se considera que el disparo de una transición tiene una duración de cero.

\section{Redes de Petri Autónomas y no-autónomas.}

Cuando una RdP describe el funcionamiento de un sistema evolucionando de una manera autónoma, es decir, cuyos instantes de disparo están entre no conocidos o no indicados, entonces podemos decir que esto es una RdP autónoma. Una RdP No-Autónoma describe el funcionamiento de un sistema cuya evolución es condicionada por eventos externos y/o tiempo. Una RdP NoAutónoma es sincronizada y/o temporizada.

\subsection{Propiedades Representativas de una RdP}

Son aquellas características que definen la correcta utilización de las RdP cuando se modela cualquier sistema. Las propiedades más importantes se encuentran vinculadas a los conceptos de limitación, redes seguras, vivacidad de la red, estados sumideros y conflictos.

Un lugar $P_{i}$ se dice que es limitado por un marcado inicial $m_{0}$, si hay un entero natural $k$, tal que para cada marcado alcanzable desde $m_{o}$, el numero de marcas en $P_{i}$ no es mayor que $k$. Una RdP es limitada por un marcado inicial $m_{0}$, si todos los lugares que la conforman, son limitados por $m_{0}$.

Una RdP se dice que es segura para un marcado inicial $m_{0}$, si para todas los marcados alcanzables, cada lugar contiene cero o una marca.

Una transición $T_{j}$ se considera viva por un marcado inicial $m_{0}$, si para cada marcado alcanzable $m_{i} \in \mu\left(m_{0}\right)$, existe una secuencia de disparo $s$ desde $m_{i}$ que contiene a $T_{j}$. Una RdP es viva para el marcado inicial $m_{0}$, si todas sus transiciones son vivas para $m_{0}$.

Un bloqueo o estado sumidero de la red, es un marcado tal que ninguna transición esta habilitada. Una RdP se dice no contiene estado sumidero o libre de bloqueos para un marcado inicial $m_{0}$, si ninguno de los marcados alcanzables $m_{i} \in \mu\left(m_{0}\right)$ es estado sumidero.

Un conflicto efectivo $K^{E}=\left\langle P_{i},\left\{T_{1}, T_{2}, \ldots\right\}, m\right\rangle$, es la existencia de un conflicto en la estructura de la red $K=\left\langle P_{i},\left\{T_{1}, T_{2}, \ldots\right\}\right\rangle$ y del marcado $m$, tal que las transiciones en el conjunto $\left\{T_{1}, T_{2}, \ldots\right\}$ son habilitadas por $m$ y el numero de marcas en $P_{i}$ es menor que la suma del peso de los arcos $P_{i} \rightarrow T_{1}, P_{i} \rightarrow T_{2}, \ldots$ 
Un conflicto general $K^{G}=\left\langle P_{i},\left\{T_{1}, T_{2}, \ldots\right\}, m\right\rangle$, es la existencia de un conflicto estructural $K=\left\langle P_{i},\left\{T_{1}, T_{2}, \ldots\right\}\right\rangle$ y del marcado $m$, tal que el numero de marcas en $P_{i}$ no es suficiente para habilitar todas las transiciones de salida de $P_{i}$ de acuerdo a su grado de activación.

\section{3. Álgebra Lineal.}

Una RdP Ordinaria no marcada o una estructura de RdP, es un grafo bipartito representado por la 4-tupla $Q=\langle P, T$, Pre, Post $\rangle$ tal que:

- $P=\left\{P_{1}, P_{2}, \ldots, P_{n}\right\}$ es el conjunto de lugares finito y no vacío;

- $T=\left\{T_{1}, T_{2}, \ldots, T_{m}\right\}$ es el conjunto de transiciones finito y no vacío;

- $P \cap T=\emptyset$, es decir que los conjuntos $P$ y $T$ son disjuntos;

- Pre $: P \times T \rightarrow\{0,1\}$ es la aplicación de la incidencia de entrada;

- Post : $T \times P \rightarrow\{0,1\}$ es la aplicación de la incidencia de salida.

$\operatorname{Pre}\left(P_{i}, T_{j}\right)$ es el peso del arco $P_{i} \rightarrow T_{j}$. El peso es 1 si el arco existe y 0 si no. post $\left(P_{i}, T_{j}\right)$ es el peso del arco $T_{j} \rightarrow P_{i}$. Pre y Post así relaciona a la transición $T_{j}$ del par $\left(P_{i}, T_{j}\right)$.

Una $\boldsymbol{R} \boldsymbol{d P}$ no marcada generalizada es definida como una RdP ordinaria no marcada, excepto

$$
\begin{aligned}
& \text { Pre }: P \times T \rightarrow \aleph \\
& \text { Post }: P \times T \rightarrow \aleph
\end{aligned}
$$

Las siguientes notaciones serán usadas:

${ }^{\circ} T_{j}=\left\{P_{i} \in P \mid \operatorname{Pre}\left(P_{i}, T_{j}\right)>0\right\}=$ el conjunto de lugares de entrada de $T_{j}$;

$T_{j}^{o}=\left\{P_{i} \in P \mid \operatorname{Post}\left(P_{i}, T_{j}\right)>0\right\}=$ el conjunto de lugares de salida de $T_{j}$;

${ }^{o} P_{i}=\left\{T_{j} \in T \mid \operatorname{Pre}\left(P_{i}, T_{j}\right)>0\right\}=$ el conjunto de transiciones de entrada de $P_{i}$;

$P_{j}^{o}=\left\{T_{j} \in T \mid \operatorname{Post}\left(P_{i}, T_{j}\right)>0\right\}=$ el conjunto de transiciones de salida de $P_{i}$.

Una $\boldsymbol{R} \boldsymbol{d P}$ marcada es un par $R=\left\langle Q, m_{0}\right\rangle$ en el cual $Q$ es una RdP no marcada y $m_{0}$ es el marcado inicial. Las condiciones de validación pueden ser 
expresadas como sigue: una transición $T_{j}$ es habilitada para un marcado $m_{k}$ si $m_{k}\left(P_{i}\right) \geq \operatorname{Pre}\left(P_{i}, T_{j}\right)$ para cada $P_{i} \in{ }^{\circ} T_{j}$.

La matriz de incidencia de entrada es $W^{-}=\left[w_{i j}^{-}\right]$donde $w_{i j}^{-}=\operatorname{Pre}\left(P_{i}, T_{j}\right)$; la matriz de incidencia de salida es $W^{+}=\left[w_{i j}^{+}\right]$donde $w_{i j}^{+}=\operatorname{Post}\left(P_{i}, T_{j}\right)$; entonces la matriz de incidencia es $W=W^{+}-W^{-}=\left[w_{i j}\right]$

Ecuación fundamental. Sea $S$ una secuencia de transiciones, el cual puede ser realizada desde el marcado $m_{i}$, el cual puede ser escrita como $m_{i} \stackrel{S}{\rightarrow}$. El vector característico de secuencia $S$, escrito como $s$, es el vector de $\mathrm{m}$ componentes cuyo componente número $j$ corresponde al número de disparos de la transición $T_{j}$ en la secuencia $S$. Si el disparo de la secuencia $S$ es tal que $m_{i} \stackrel{S}{\rightarrow} m_{k}$, entonces la ecuación fundamental es obtenida.

$$
m_{k}=m_{i}+W \cdot s
$$

El conjunto de alcanzabilidad de una $\boldsymbol{R d P}$ es el conjunto de todos los marcados alcanzables desde $m_{0}$ disparando solo transiciones habilitadas; este conjunto es denotado por $\mathbb{R}\left\langle Q, m_{0}\right\rangle$. Una secuencia de transiciones de disparo de una $\operatorname{RdP}\left(Q, m_{0}\right)$, es una secuencia de transición $S=T_{i}, T_{j}, \ldots, T_{k}, \ldots$ tal que $m_{0} \stackrel{T_{i}}{\rightarrow} m_{1} \stackrel{T_{j}}{\rightarrow} m_{x} \stackrel{T_{k}}{\rightarrow} \ldots$

El conjunto de todas las secuencias de disparo es llamado el lenguaje $\mathcal{L}\left(Q, m_{0}\right)$ :

$$
\mathcal{L}\left(Q, m_{0}\right)=\left\{S=T_{i}, T_{j}, \ldots, T_{k}, \ldots \wedge m_{0} \stackrel{T_{i}}{\rightarrow} m_{1} \stackrel{T_{j}}{\rightarrow} m_{x} \stackrel{T_{k}}{\rightarrow} \ldots\right\}
$$

\subsection{Redes de Petri No-Autónomas}

Se han revisado hasta el momento las RdP autónomas, las cuales permiten un análisis cualitativo, ahora se presentan la extensión de las RdP en donde es posible describir no solo "que pasa" sino también "cuando pasa" un hecho. Las RdP No-autónomas habilitarán el sistema a ser modelado cuyos disparos están sincronizados con eventos externos y/o cuyas evoluciones son dependientes del tiempo.

\section{Redes de Petri Sincronizadas}

En una RdP autónoma, sabemos que una transición puede ser disparada si ésta es habilitada, pero no conocemos cuando ésta podría ser disparada. En 
una RdP sincronizada, un evento es asociado con cada transición, y el disparo de esta transición ocurrirá si la transición es habilitada, cuando los eventos asociados ocurren. Los eventos externos corresponden a un cambio en el estado de la palabra externa (incluyendo el tiempo); por el contrario, un cambio en el estado interno, un cambio en el marcado, podría ser llamado un evento interno. La ocurrencia de un evento no tiene duración.

Una RdP Sincronizada es un Triple $\langle R, E$, Sync $\rangle$ tal que:

$R$ es una RdP marcada.

$E$ es un conjunto de eventos externos,

Sync es una función del conjunto $T$ de las transiciones de $R$ de $E \cup\{e\}$, en el cual $e$ es siempre el evento que ocurre o vacío .

$E=\left\{E^{1}, E^{2}, \ldots\right\}$ es un conjunto de eventos externos. La notación $E^{i}$ corresponde al nombre de un número de evento externo. La notación $E_{j}$ corresponde a el evento asociado a la transición $E_{j}$.

\section{Redes de Petri Interpretadas}

Una Red de Petri Interpretada exhibe características tales como sincronización, lugares temporizados ( $\mathrm{P}$-timed) y tiene una parte para procesamiento de datos. Las entradas están asociadas con las transiciones y las salidas están asociadas con los lugares. Como se puede ver en la figura 3.2, el evento $E_{j}$ y la condición $C_{j}$ son asociados con la transición $T_{j}$. La condición $C_{j}$ es una función booleana dependiendo de la parte de procesamiento de datos y el ambiente. El evento $E_{j}$ está entre un evento externo derivado del ambiente o un evento ocurriendo siempre $e$. la transición $T_{j}$ sera disparada si:

la transición $T_{j}$ esta habilitada

y si la condición $C_{j}$ es verdadera,

cuando el evento $E_{j}$ ocurra.

El producto $R_{j}=E_{j} \cdot C_{j}$ es llamada receptividad de la transición $T_{j}$. La acción denotada en la figura $O_{i}^{*}, B_{i}^{*}$, y $A_{i}$ son asociadas con el lugar $P_{i}$. Cuando una marca es depositada en el lugar $P_{i}$, en el instante $t$, la operación $O_{i}^{*}$ es realizada y la acción impulso $B_{i}^{*}$ es enviado al ambiente. La salida booleana $A_{i}$ tiene el valor booleana 1 tanto tiempo como hay una marca en el lugar $P_{i}$.

Una RdP Interpretada de control exhibe las siguientes cinco características (1 a 3 necesariamente y 4 y 5 posiblemente). 

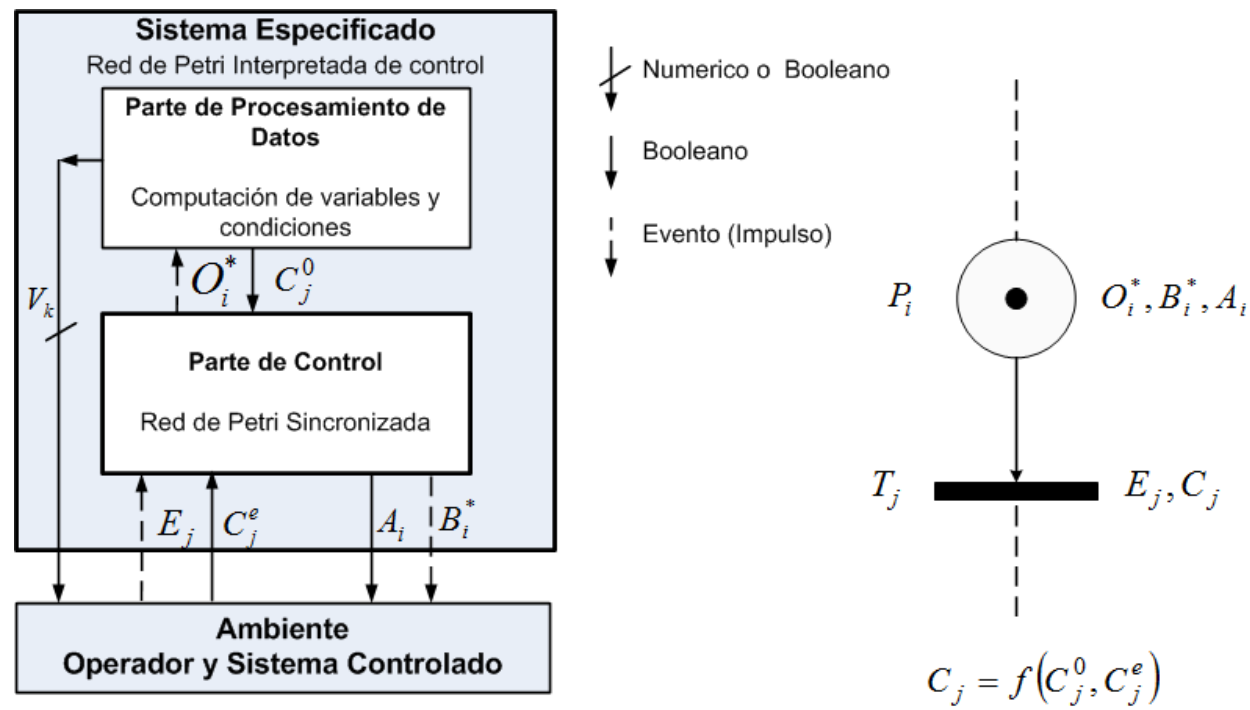

Figura 3.2: Red de Petri Interpretada de Control

1. Es sincronizada a eventos externos y estable

2. Es segura

3. Es determinística

4. Tiene una parte de procesamiento de datos cuyo estado es definido por un conjunto de variables $V=\left\{V_{1}, V_{2}, \ldots\right\}$. Este estado es modificado por operaciones $O_{i}^{*}$ los cuales son asociados a los lugares. Éste determina el valor de los predicados $C_{j}^{0}$.

5. Recibe información booleana $C_{j}^{e}$ del ambiente. Envía acciones de nivel $A_{j}$ y acciones impulso $B_{j}$, asociadas con los lugares, al ambiente.

\section{Redes de Petri Temporizadas}

Las RdP temporizadas son útiles para evaluar comportamiento y habilita a un sistema a ser descrito cuando su funcionamiento es dependiente del tiempo. Por ejemplo, un cierto tiempo puede durar entre el inicio y el final de una operación. Si un marcado en un cierto lugar indica que esta operación esta en progreso, una RdP temporizada permite al tiempo ser tenido en cuenta. Hay dos métodos principales para modelar el tiempo: Entre el tiempo asociado con los lugares (P-timed) o el tiempo asociado con las transiciones (T-timed). 
Redes de Petri P-timed. Un tiempo $d_{i}$ de valor cero si no es especificado, es asociado con cada lugar $P_{i}$. Debemos considerar el caso donde $d_{i}$ es un valor constante, pero en general puede ser variable. Una RdP P-timed es el par $\langle R$, Tempo $\rangle$ tal que:

$R$ es una RdP marcada;

Tempo es una función del conjunto de lugares $P$ del conjunto de números racional cero o positivo. Tempo $\left(P_{i}\right)=d_{i}=$ tiempo asociado con el lugar $P_{i}$.

Cuando una marca es depositada en un lugar $P_{i}$, esta marca debe mantenerse en este lugar al menos por un tiempo $d_{i}$. Esta marca se dice que es no disponible para este tiempo. cuando el tiempo $d_{i}$ ha transcurrido, entonces la marca se convierte en disponible.

Redes de Petri T-timed. Un tiempo $d_{j}$, de valor cero si no es especificado, es asociado con la transición $T_{j}$. Una RdP T-timed es el par $\langle R, T$ empo $\rangle$ tal que:

$R$ es una RdP marcada;

Tempo es una función del conjunto de transiciones $T$ del conjunto de números racional cero o positivo. $\operatorname{Tempo}\left(T_{j}\right)=d_{j}=$ tiempo asociado con la transición $T_{j}$.

Cuando una transición $T_{j}$ se convierte en habilitada, esta no es disparada inmediatamente. Esta será disparada cuando el tiempo $d_{j}$ haya transcurrido después de la habilitación, excepto en caso de un conflicto. Una marca puede tener dos estados: puede ser reservada para el disparo de la transición $T_{j} 0$ puede no ser reservada.

Redes de Petri Estocásticas. Una RdP estocástica puede ser considerada como una RdP Temporizada en el cual los tiempos tienen valores estocásticos. Una RdP Estocástica es el par $\langle R$, Rate $\rangle$ tal que:

$R$ es una RdP marcada;

Rate es una función desde el conjunto de transiciones $T$ al conjunto de números reales positivos finitos. $\operatorname{Rate}\left(T_{j}\right)=\mu_{j}=$ rata de disparo asociado con $T_{j} . \mu$ representa la función de probabilidad de disparo.

\subsection{Redes de Petri Autónomas}

La Red de Petri continua es un modelo en el cual el numero de marcas en el lugar es representado por números reales en vez de enteros. 


\section{Redes de Petri Continuas.}

consideremos un $\operatorname{RdP} R$, (autónoma, discreta, ordinaria o generalizada) definida por su grafo $Q$ (lugares, transiciones, arcos) y su marcado $m$. Consideremos aplicar una transformación, la cual consiste en dividir cada marca, en $k$ partes iguales (sin cualquier otra modificación de la $\mathrm{RdP}$ ) y en donde se consigue el disparo de una transición $T_{j}$, removiendo una de estas $k$ partes (denominados también tokens) de un lugar de entrada $P_{i}$, para adherirlo, después del disparo, a un lugar de salida.

Una RdP continua autónoma es una 5-tupla $R=\left\langle P, T\right.$, Pre, Post, $\left.m_{0}\right\rangle$ tal que:

- $P=\left\{P_{1}, P_{2}, \ldots, P_{n}\right\}$ es el conjunto de lugares finito y no vacío;

- $T=\left\{T_{1}, T_{2}, \ldots, T_{m}\right\}$ es el conjunto de transiciones finito y no vacío;

- $P \cap T=\emptyset$, es decir que los conjuntos $P$ y $T$ son disjuntos;

- Pre $: P \times T \rightarrow Q_{+}$es la aplicación de la incidencia de entrada;

- Post $: T \times P \rightarrow Q_{+}$es la aplicación de la incidencia de salida;

- $m_{0}: P \rightarrow R_{+}$es el marcado inicial.

El peso de los arcos podrían ser definidos como números reales. El marcado de un lugar debe ser un número real desde que éste puede cambiar continuamente. Los lugares y transiciones son representados por una doble linea. En una RdP continua, el grado de habilitado de una transición $T_{j}$ para el marcado $m$, denotado por $q \circ q\left(T_{j}, m\right)$ es un numero real $q$ tal que

$$
q=\min _{i: P_{i} \in{ }^{\circ} T_{j}}\left(\frac{m\left(P_{i}\right)}{\operatorname{Pre}\left(P_{i}, T_{j}\right)}\right)
$$

Si $q>0$, transición $T_{j}$ es habilitada; esto para decir $q-e n a b l e$.

\section{Redes de Petri Híbridas}

Una RdP híbrida está compuesta por lugares y transiciones continúas (C) y lugares y transiciones discretas (D). Como se observa en la figura 3.3, la representación de los lugares y transiciones de la parte discreta y continua es 

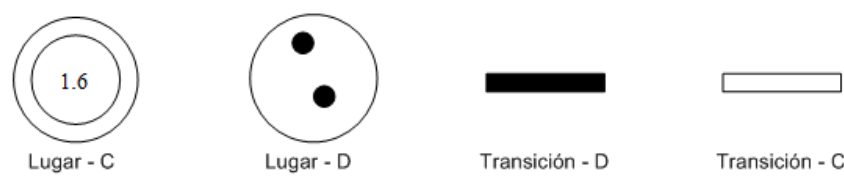

Figura 3.3: Lugares y Transiciones de RdP Híbrida

diferente, además, el marcado de un lugar continuo es representado por un número real a diferencia de un lugar discreto que mantiene los puntos negros.

Una RdP Híbrida autónoma marcada es una séxtupla $R=\left\langle P, T\right.$, Pre, Post, $\left.m_{0}, h\right\rangle$ cumpliendo las siguientes condiciones:

- $P=\left\{P_{1}, P_{2}, \ldots, P_{n}\right\}$ es un conjunto de lugares finito y no vacío;

- $T=\left\{T_{1}, T_{2}, \ldots, T_{m}\right\}$ es un conjunto de transiciones finito y no vacío;

- $P \cap T=\emptyset$, es decir que los conjuntos $P$ y $T$ son disjuntos;

- $h: P \cup T \rightarrow\{D, C\}$, llamada función Híbrida, indica para cada nodo si éste es un nodo discreto (conjunto $P^{D}$ y $T^{D}$ ) o continuo (conjunto $P^{C}$ y $T^{C}$ ) .

- Pre $: P \times T \rightarrow \mathcal{Q}_{+}$o $\mathcal{N}$ es la aplicación de la incidencia de entrada;

- Post $: T \times P \rightarrow \mathcal{Q}_{+}$o $\mathcal{N}$ es la aplicación de la incidencia de salida;

- $m_{0}: P \rightarrow R_{+} \circ \mathcal{N}$ es el marcado inicial.

En las definiciones de Pre, Post y $m_{0}, \mathcal{N}$ corresponde al caso donde $P_{i} \in P^{D}$ , y $\mathcal{Q}_{+} \circ \mathcal{R}_{+}$corresponde al caso donde $P_{i} \in P^{C}$. Una transición discreta en una RdP híbrida es habilitada si cada lugar $P_{i}$ en ${ }^{o} T_{j}$ encuentra la condición $m\left(P_{i}\right) \geq \operatorname{Pre}\left(P_{i}, T_{j}\right)$. Una transición continua en una RdP híbrida es habilitada si cada lugar $P_{i}$ en ${ }^{o} T_{j}$ encuentra la condición $m\left(P_{i}\right) \geq \operatorname{Pre}\left(P_{i}, T_{j}\right)$, si $P_{i}$ es un lugar discreto (D), o $m\left(P_{i}\right)>0$, si $P_{i}$ es un lugar continuo $(\mathrm{C})$.

La Matriz de Incidencia de una RdP Híbrida puede ser escrita como:

$$
W=\left[\begin{array}{cc}
W^{D} & 0 \\
W^{C D} & W^{C}
\end{array}\right]
$$

donde $W^{D}$ corresponde a los arcos entre nodos discretos, $W^{C}$ a los arcos entre nodos continuos, y $W^{C D}$ a los arcos entre los lugares $C$ y transiciones $D$. Los arcos entre los lugares $D$ y transiciones $C$ corresponden a la submatriz 0 . 
- Las funciones Pre y Post deben mantener los siguientes criterios: si $P_{i}$ y $T_{j}$ son un lugar y una transición tal que $h\left(P_{i}\right)=D$ y $h\left(T_{j}\right)=C$, entonces $\operatorname{Pre}\left(P_{i}, T_{j}\right)=\operatorname{Post}\left(P_{i}, T_{j}\right)$ debe ser verificada.

Esta última condición establece que un arco debe unir a una transición- $\mathrm{C}$ a un lugar-D tan pronto como un arco recíproco exista. Esto asegura que el marcado de un lugar-D, es un entero cualquiera que sea la evolución que ocurra.

Una RdP Híbrida Marcada es un par $H=\left\langle H^{*}, m_{0}\right\rangle$ donde $H^{*}$ es una RdP Híbrida no marcada y $m_{0}$ es el marcado inicial. El marcado inicial de un lugar-D es un entero positivo o nulo mientras que el marcado inicial de un lugar- $\mathrm{C}$ es un número real positivo o nulo.

Una RdP Híbrida Generalizada es definida como la RdP Híbrida no marcada, excepto que:

- Si $P_{i}$ es un lugar-D, $\operatorname{Pre}\left(P_{i}, T_{j}\right)$ y $\operatorname{Post}\left(P_{i}, T_{j}\right)$ son enteros positivos.

- Si $P_{i}$ es un lugar-C, $\operatorname{Pre}\left(P_{i}, T_{j}\right)$ y $\operatorname{Post}\left(P_{i}, T_{j}\right)$ son números reales positivos.

Una matriz de incidencia es asociada con cada red:

$$
W=\left[W_{i j}\right]_{n \times m}, \text { donde } W_{i j}=\operatorname{Post}\left(P_{i}, T_{j}\right)-\operatorname{Pre}\left(P_{i}, T_{j}\right)
$$

Una Transición-D es habilitada si cada lugar $P_{i}$ en ${ }^{\circ} T$ verifica que el $M\left(P_{i}\right) \geq$ $\operatorname{Pre}\left(P_{i}, T_{j}\right)$. Se puede ver que esta definición no separa el caso donde $P_{i}$ es un lugar-D de un caso donde $P_{i}$ es un lugar-C.

Una Transición-C es habilitada si las dos condiciones siguientes son encontradas:

1. Para cada lugar-D $P_{i}$ en ${ }^{\circ} T_{j}, M\left(P_{i}\right) \geq \operatorname{Pre}\left(P_{i}, T_{j}\right)$.

2. Para cada lugar-C $P_{i}$ en ${ }^{\circ} T_{j}, . M\left(P_{i}\right)>0$.

Para una transición-C, la clase de lugar precediendo la transición, debe ser especificada porque las condiciones que habilitan son diferentes acorde a entre si esta es lugar-C o lugar-D.

Sea $S$ una secuencia de disparo y $s$ sea el vector característico de $S$. La dimensión del vector $s$ es igual a el número $m$ de transiciones. El j-ésimo componente de $m$ representa el número de disparos de transiciones $T_{j}$ y será deno- 
CAPÍTULO 3. FUNDAMENTOS DE REDES DE PETRI

tado por $n_{j}$. Si $T_{j}$ es una transición-D, entonces $n_{j}$ es un entero y si $T_{j}$ es una transición-C, entonces $n_{j}$ es un número real.

Un marcado $m$ puede ser deducido de un marcado $m_{0}$ debido a una secuencia $S$, usando la relación fundamental:

$$
M=M_{0}+W \cdot \underline{S}
$$

La relación fundamental de una RdP Híbrida es idéntica con la relación fundamental de una RdP Discreta. Entonces, se puede deducir que cada propiedad de RdP Discretas resultante de esta relación puede ser transpuesta a RdP Híbridas.

Esta parte de procesamiento de datos es definida por un conjunto de variables . Este estado es modificado por operaciones $O=\left\{O_{1}, O_{2}, \ldots.\right\}$, asociadas a los lugares. Estas determinan el valor de las condiciones $C=\left\{C_{1}, C_{2}, \ldots.\right\}$ que son asociadas a las transiciones. 


\section{Capítulo 4}

\section{Construcción del Modelo y Diagnosticador usando RdP para FD de SED's}

A lo largo de los años de investigación sobre diagnóstico de fallos en SED's, básicamente se han utilizado varias herramientas de modelado en el campo de la Automática, entre las más importantes que se plantean son los Lenguajes Regulares, las MEF's y por su excelente capacidad de modelado y recursos matemáticos, están las Redes de Petri (RdP); En los capítulos iniciales se dio a conocer las dificultades y limitaciones que se presentan cuando se hace diagnóstico de fallos mediante las técnicas diferentes a RdP, la dificultad más representativa es la explosión combinacional, donde prácticamente se vuelve irrealizable la aplicación de esta técnica a un proceso de grado medio de complejidad, ratificado en los trabajos desarrollados por [18][33][86]. Una de las mejores alternativas para reducir estas dificultades, es el empleo de las RdP en el diagnóstico de fallos de SED's, con buenos resultados publicados en [84][78][35], Incluso, se puede hablar que han habido trabajos donde mezclan los beneficios de MEF's y RdP [11][15][101] por lo tanto, en este capítulo todo el desarrollo del modelo y diagnosticador se hace con RdP. Ahora se reduce la complejidad y se interpreta mejor la dinámica del sistema, llevando a que el diagnosticador tenga un modelo de RdP referente mas preciso y flexible.

En este capítulo se presenta una metodología para la construcción del modelo y diagnosticador de FD de un SED's. Debemos asumir que el sistema de 
CAPÍTULO 4. CONSTRUCCIÓN DEL MODELO Y DIAGNOSTICADOR USANDO RDP PARA FD DE SED'S

interés está compuesto de elementos físicos y está dotado con un conjunto de sensores. Iniciando desde el modelo de RdP de cada componente y las lecturas que nos entregan los sensores, presentamos un procedimiento sistemático para generar un modelo de RdP general, que captura la interacción de sus componentes y la información suministrada por los sensores. Este modelo es refinado para eliminar transiciones no observables y así construir el diagnosticador. En el modelo se tiene en cuenta el comportamiento normal y de fallo del sistema. A continuación presentamos un algoritmo para construir un modelo y diagnosticador, para ilustrar este procedimiento se utiliza un ejemplo del un sistema de Aire acondicionado y ventilación (HVAC), por último, se presentan algunas observaciones y conclusiones generales del algoritmo.

\subsection{Características Importantes de un FDI en SED's}

Después de haber hecho una revisión de los desarrollos más importantes de los algoritmos de diagnóstico de fallos en SED's, se puede deducir unas características esenciales cuando se piensa en evaluar o valorar una técnica de diagnóstico de fallos. Son importantes porque en los capítulos siguientes se hace el desarrollo del diagnóstico de fallos mediante RdP y se espera que todas estas características sean tenidas en cuenta en la medida de lo posible. A continuación se presentan las características a criterio del autor de este documento.

- Proceso de construcción sistemática. Se debe tener una herramienta que sea fácil de implementar, donde se considere unos pasos de construcción sencillos y prácticos.

- Explosión combinacional. Como se vio anteriormente éste es uno de los problemas que abarca a la mayoría de las técnicas existentes, por eso la herramienta tiene que ser bien implementada en cualquier SED's, sin importar el tamaño.

- Diagnóstico On-line. El algoritmo debe mantener una observación instantánea del proceso, detectando de manera inmediata los fallos.

- Supervisión. El diagnosticador debe ser una herramienta de ayuda al operador del proceso, que ubique los fallos pero al mismo tiempo informe del estado del proceso, ya que, el proceso sigue funcionando así haya 
ocurrido un fallo y se necesita tomar decisiones de paro en cierto estado del proceso.

- Tipo de Fallos a Detectar. La herramienta debe detectar uno o varios fallos simultáneos, sin importar el orden de aparición, en un proceso real, no se puede predecir el orden de aparición de los fallos. Otro aspecto importante a tener en cuenta, es que hay fallos que dependen de otro fallo, por lo tanto, el diagnosticador debe estar en capacidad de encontrar este tipo de fallos.

- Flexibilidad. Esta característica es importante. Como es habitual en los procesos industriales, es necesario hacer cambios en su estructura, adicionar componentes, etc. Lo anterior obliga a que existan nuevas fuentes de fallo y por lo tanto, la herramienta de FD tenga que ser adecuada a estas exigencias, en conclusión, debe existir la flexibilidad necesaria para adaptarse a las transformaciones del sistema.

Puede parecer altas las exigencias a los algoritmos de diagnóstico de fallos, pero en realidad se esta hablando de técnicas que esperan darle seguridad no solo a un proceso, si no también a vidas humanas, además, representando altas cantidades de dinero, así que siempre que se piense en diseñar un algoritmo, se debe tener un mínimo de exigencias para con los aspectos de operación, construcción y mantenimiento.

\subsection{Construcción del Modelo}

La teoría fundamental de las RdP se basa en la identificación individual de los componentes del sistema (SED's) y la relación entre ellos, en el modelo se debe incluir el comportamiento normal del proceso, junto con el comportamiento de fallo, similar al presentado por [86] [78]. Sea $R=\left(P, T\right.$, Pre, Post, $\left.m_{0}\right)$, la RdP descrita en el capítulo 3 que representa el modelo de eventos discretos del sistema a diagnosticar. Las transiciones que componen el sistema son clasificadas en el conjunto de transiciones observables $T_{o}$ y el conjunto de transiciones no observables $T_{u o}, T=T_{o} \cup T_{u o}$. Cuando se hace referencia a observable, quiere decir que estas transiciones pueden ser observadas por cualquier agente del sistema, las cuales son dadas por los eventos de control $T_{C}$ (comandos del supervisor) y por la instrumentación implementada en el proceso, tales como las 
CAPÍTULO 4. CONSTRUCCIÓN DEL MODELO Y DIAGNOSTICADOR USANDO RDP PARA FD DE SED'S

transiciones dadas por los sensores $T_{S}, T_{o}=T_{C} \cup T_{S}$. No observable se refiere a transiciones que suceden desde cualquier estado o lugar del sistema $P_{i}$ y no está contemplado en el modelo que describe el funcionamiento del sistema. En las transiciones no observables es donde están incluidas las transiciones de fallo $T_{f}, T_{f} \subseteq T_{u o}$. El objetivo que se plantea cualquier sistema de diagnóstico de fallos es el de identificar las $T_{u o}$, ya que las $T_{o}$ puede ser identificadas fácilmente por el sistema; Las $T_{f}$ se clasifican en conjuntos disjuntos correspondientes a los diferentes tipos de fallo que se pueden presentar en el sistema, siendo importante poder distribuir los fallos en grupos que permita facilitar su identificación al sistema de diagnóstico, por lo tanto, el conjunto de las transiciones de fallo $T_{f}$ esta compuesto de los diferentes subconjuntos de fallo dados en el proceso, $T_{f}=T_{f_{1}} \cup \ldots \cup T_{f_{N}} . N$ es el número de componentes que integran el sistema y sea $\Pi_{f}$ la distribución de fallos, dicha partición viene justificada por:

- No es necesario identificar la transición de fallo de forma particular, es preferible de un conjunto de fallos, identificar alguno que conduzca a diagnosticar el fallo, suceda el que suceda, esto le permite al sistema ser más práctico.

- La instrumentación inadecuada que lleva el proceso, que hace imposible de forma simple diagnosticar cada posible fallo.

Por consiguiente, cuando un fallo de tipo $F_{i}$ ha ocurrido, significa que alguna transición del conjunto $T_{f_{i}}$ ha ocurrido.

El primer paso es construir el modelo de Red de Petri de cada uno de los componentes del proceso, suponiendo que el sistema tiene $N$ componentes individuales, sea la expresión:

$$
R_{i}=\left(P_{i}, T_{i}, \text { Pre, } \text { Post }, m_{0}\right)
$$

$R_{i}$ representa la $\mathrm{RdP}$ marcada del componente $i, P_{i}=\left\{P_{1}, P_{2}, \ldots, P_{n}\right\}$ es el conjunto de lugares del componente $i, T_{i}=\left\{T_{1}, T_{2}, \ldots, T_{m}\right\}$ es el conjunto de transiciones del componente $i$; Pre $: P \times T \rightarrow\{0,1\}$ es la aplicación de la incidencia de entrada; Post $: T \times P \rightarrow\{0,1\}$ es la aplicación de la incidencia de salida. Es importante notar que se debe poseer un conocimiento profundo del proceso, ya que, en el modelo se debe incluir el comportamiento normal y de fallo de cada componente y mantener la sincronía de funcionamiento del 
proceso completo. Para diferenciar los lugares de fallo y las transiciones de fallo, éstas se deben representar con círculos y barras sombreados respectivamente.

\subsection{Operación de Integración y Refinamiento del Modelo.}

Operación de integración se refiere a buscar representar, a través de un único modelo de RdP, el comportamiento general del SED's, donde se incluyan los diferentes modelos de RdP de los componentes del proceso. Sea $\widetilde{Q}=$ $\left(\widetilde{P}, \widetilde{T}, \widetilde{P r e}, \widetilde{\text { Post }}, m_{0}\right)$ la denotación de la operación de integración de los modelos de los componentes $R_{i}, i=1, \ldots, N$. En este modelo se integra el comportamiento normal y de fallo del sistema, descrito y modelado en el modelo individual de los componentes. Desde cada lugar $p_{i}$ del modelo, suceden las transiciones observables $T_{o}$ de funcionamiento normal del sistema $T_{N}$ (dadas por el sistema de control $T_{C}$ ), y las transiciones no observables $T_{u o} . \widetilde{P}$ es el conjunto de lugares y $\widetilde{T}$ es el conjunto de transiciones que agrupa a los $N$ componentes del sistema:

$$
\widetilde{P}=\bigcup_{i=1}^{N} p_{i} \quad y \quad \widetilde{T}=\bigcup_{j}^{N} T_{j}
$$

\section{Construcción del Modelo de Integración.}

- Paso 1. Establecer el conjunto de fallos $F$, basado en los fallos definidos para cada componente del sistema, $F=\left\{F_{1}, F_{2}, \ldots, F_{N}\right\}$.

- Paso 2. Definir el lugar de inicio $p_{1}$. Representa a todos los lugares de inicio de cada uno de los componentes del sistema. $P_{1}=\left\{P_{0}^{1}, P_{0}^{2}, \ldots, P_{0}^{N}\right\}$.

- Paso 3. Asignar un marca al lugar de inicio $p_{0}$, ella es quien otorga el inicio a la evolución del sistema representado.

- Paso 4. Construir la rama de funcionamiento normal $R_{N}$ del sistema. Basado en el funcionamiento individual de cada componente del sistema, se construye una rama, llamada de funcionamiento normal, para describir la interacción y evolución de los componentes, siguiendo las pautas establecidas por las transiciones dadas por el controlador. En cada lugar se debe ir representando el estado actual de cada componente. Las transiciones de la RdP están compuestas solo por los comandos del controlador $T_{C}$. 
- Paso 5. Agregar los lugares de fallo $P_{f}$. En el paso 1, se definió un conjunto de fallos, para cada fallo del sistema, se adiciona al modelo general para cada fallo, un lugar de fallo.

- Paso 6. Agregar las transiciones de fallo $T_{f}$. En cada lugar de la RdP general se puede presentar la evolución de los fallos enunciados en los modelos de RdP de cada componente, es decir, que desde cada lugar $P_{i}$ se adiciona una transición de fallo $T_{f}$ la cual conduce a los lugares de fallo $P_{f}$ agregados en el paso anterior. $\forall p_{i}: p_{i} \in P \rightarrow \exists\left(P_{i}, T_{j}\right) \in F$, donde $F$ es el conjunto de fallos del sistema.

Los lugares de fallo agregados al modelo, se constituyen en lugares de bloqueo de la RdP, conocidos como lugares sumidero. Dado que la construcción de este algoritmo de FD es sistemático, esto no representa aún un problema, ya que, quien realmente va a cumplir la función de supervisor es el diagnosticador y para ésta parte, la RdP habrá sufrido las transformaciones necesarias, donde se corrigen estos bloqueos y se garantiza poder tener una RdP viva, es decir, libre de bloqueos. El funcionamiento del sistema puede ser de ciclo abierto o cerrado, esto es definido por la dinámica de funcionamiento del sistema representado.

El modelo general está compuesto por transiciones observables $T_{o}\left(T_{C}\right)$ y no observables $T_{u o}\left(T_{f}\right)$. Es necesario considerar únicamente la parte observable del modelo general del sistema $\widetilde{Q}$, por lo tanto, $\widetilde{Q}=\left(\widetilde{P}, \widetilde{T}, \widetilde{\text { Pre }}, \widetilde{\text { Post }}, m_{0}\right)$ debe ser transformada a un modelo general refinado $Q=\left(P, T\right.$, Pre, post, $\left.m_{0}\right)$, el cual quedará solo compuesto de transiciones y lugares observables. Para realizar esta transformación o refinamiento del modelo general, se deben sustituir las transiciones de fallo por transiciones de lecturas de los sensores $T_{S}$, que son $T_{0}$. Para ello se propone el siguiente algoritmo.

\section{Refinamiento del Modelo General}

- Paso 1. Identificar los sensores $S$ con que cuenta el sistema. La idea fundamental del algoritmo es aprovechar la información disponible del sistema, con esta poder identificar la aparición de posibles condiciones anormales del sistema.

- Paso 2. Construir el conjunto discreto de posibles salidas de los sensores que componen el sistema. Dado el conjunto $N$ sensores del sistema de 
Tabla 4.1: Tabla de salidas de Sensores

\begin{tabular}{|cc|}
\hline$S_{1}$ & $S_{2}$ \\
\hline \hline$\overline{L_{1}}$ & $\overline{L_{2}}$ \\
\hline$\overline{L_{1}}$ & $L_{2}$ \\
\hline$L_{1}$ & $\overline{L_{2}}$ \\
\hline$L_{1}$ & $L_{2}$ \\
\hline
\end{tabular}

interés, identificar sus salidas y con ellas construir la combinación posible de las lecturas sensoriales. En el conjunto de posibles salidas de sensor $Y$, serán tantas combinaciones como sensores compongan el sistema, es decir, $|Y|=2^{S}$. Para hacer una mejor ilustración de la construcción de las posibles salidas de sensores, supongamos que el sistema posee dos sensores, su estado discreto de cada sensor es $L$ o $\bar{L}\left(\begin{array}{lll}1 & 0 & 0\end{array}\right)$ según corresponda. Para el ejemplo tendremos $2^{2}=4$ combinaciones de salidas de sensores, representados en la tabla 4.1. Las combinación dada anteriormente se convierten en las entradas de la tabla de integración de sensores.

- Paso 3. Definir las salidas de la tabla de integración. Para cada uno de los lugares de la rama de funcionamiento normal de la RdP que representa el sistema, se adiciona una columna a la tabla. Cada lugar representará el estado actual de los diferentes componentes del sistema, por ejemplo, el lugar $P_{0}$ representa a los elemento $X, Y$ y $Z$. Entonces, $P_{0}=(X, \overline{Y, Z})$, muestra que el elemento $X$ esta en un estado positivo y tanto $Y$ como $Z$, mantienen un estado negativo.

- Paso 4. Construir la tabla de integración de sensores $h_{j}=\widetilde{P} \rightarrow \widetilde{Y}_{j}, j=$ $1, \ldots, N$, donde $\tilde{Y}_{j}$ denota el conjunto discreto de posibles salidas del $j$ ésimo sensor, se define:

$$
Y=\prod_{j=1}^{N} Y_{j}
$$

y sea $h: \widetilde{P} \rightarrow Y$ la denotación de la tabla de integración de sensores definida como se indica a continuación: $h(P)=\left(h_{1}(P), h_{2}(P), \ldots, h_{N}(P)\right)$. Este proceso se refiere a basados en el conjunto de posibles salidas de sensores, los disponemos a cada lugar de la rama de funcionamiento normal del modelo general, es decir, para cada lugar de la rama, existe una 
combinación de salidas sensoriales que representa el estado normal, llamada $Y_{j N}$, y las restantes salidas de lectura sensorial, pueden conducir a identificar un fallo, identificadas como salidas de sensores en estado de fallo $Y_{j F}$, o salidas que su lectura no entrega ninguna información válida, de cara a la identificación de fallos $Y_{j X} . Y_{j}=Y_{j N} \cup Y_{j F} \cup Y_{j X}$. Por lo tanto, en cada lugar del modelo se pueden definir las salidas de sensores como $N$ (normal), $F$ (fallo) y $X$ (no determinado).

- Paso 5. Eliminar los lugares de fallo no alcanzables. En el modelo general también existen lugares de fallo que no se pueden identificar, debido a que la información con que se cuenta, basado en las salidas de los sensores, no permite habilitar nunca ese marcado, esto se conoce como lugares no alcanzables y deben ser eliminados al realizar el refinamiento del modelo.Un lugar $p$ es no alcanzable cuando por las condiciones de funcionamiento del sistema nunca se llegará a dar o presentar, es decir, su marcado en la RdP no es alcanzable,

$$
\forall p_{i}: p_{i} \in P \rightarrow M\left(p_{i}\right) \notin R\left(Q, M_{0}\right)
$$

Finalmente, el modelo de integración refinado está compuesto de lugares que componen el funcionamiento síncrono de los componentes del sistema, lugares normales $P_{i}=p_{j}^{1} \cup p_{j}^{2} \cup \ldots \cup p_{j}^{n}$ unido a los lugares de fallo $P_{f}$ dados en $\Pi_{f}, P=P_{N} \cup P_{F}$. Las transiciones $T$ estarán compuestos de eventos de control o supervisor $T_{C}$ unido a los eventos dados en la tabla de integración de sensores $T_{S}, T=T_{C} \cup T_{S}$, los cuales hacen que el modelo general refinado esté compuesto solo de transiciones observables.

\subsection{El Diagnosticador y la Diagnosticabilidad}

Sea $Q=\left(P, T\right.$, Pre, Post, $\left.M_{0}\right)$, la RdP descrita en el apartado anterior, que representa el modelo de eventos discretos del sistema a diagnosticar, la cual está compuesta sólo de transiciones observables $T_{o}$ y lugares observables $P_{o}$, Con ello se garantiza sencillez y robustez en el diagnosticador. En el contexto de la metodología desarrollada en esta investigación se deben realizar los siguientes supuestos: 
- Existe una transición definida en cada lugar $p \in P$, por lo tanto, la red de Petri no alcanzará ningún lugar sumidero, evitando que la red quede en un estado de bloqueo.

- No existe en $Q$ transiciones no observables $T_{u o}$. Esta condición esta garantizada, gracias al desarrollo de un modelo general refinado, es decir, que solo contiene transiciones observables.

Sea $T_{f}$ la transición final de una secuencia $S$ definimos:

$$
\mathcal{L}\left(T_{f_{i}}\right)=\left\{S T_{f} \in L: T_{f} \in T\right\}
$$

Es decir, $\mathcal{L}\left(T_{f_{i}}\right)$ denota el conjunto de todos las secuencias de $L$ (Lenguaje que representa el comportamiento del SED's), que acaba en una transición de fallo, perteneciente a la clase $T_{f_{i}}$, considérese $T_{f} \in T$ y $S \in T^{*}$, utilizaremos la notación $T \in S$ para denotar de que $T$ es una transición de la secuencia $S$, también escribimos $T_{f} \in T$ para cualquier $T_{f}$.

\subsubsection{Construcción del Diagnosticador}

En el proceso de construcción del diagnosticador, lo primero a realizar es definir el conjunto de las etiquetas de fallo. Basado en los fallos que fueron definidos en el modelo general, $\triangle f=\left\{F_{1}, F_{2}, \ldots, F_{m}\right\},\left|\Pi_{f}\right|=m$. El conjunto de etiquetas general del diagnosticador $\Delta$, esta compuesto por etiquetas normales $\Delta N$ y etiquetas de fallo $\triangle F, \triangle=\{\Delta N\} \cup\{\triangle F\}$. El diagnosticador para $Q$ es una RdP. Sea $Q_{d}=\left(P_{d}, T_{d}\right.$, Pre, Post, $\left.m_{0}, P_{0}, T_{0}, T_{\text {end }}\right)$ la RdP diagnosticadora (RdPD) que representa el diagnosticador, tal que:

- $P_{d}=\left\{P_{1}, P_{2}, \ldots, P_{n}\right\}$ es el conjunto de lugares finito y no vacío;

- $T_{d}=\left\{T_{1}, T_{2}, \ldots, T_{m}\right\}$ es el conjunto de transiciones finito y no vacío;

- $P \cap T=\emptyset$, es decir que los conjuntos $P_{d}$ y $T_{d}$ son disjuntos;

- Pre $: P \times T \rightarrow\{0,1\}$ es la aplicación de la incidencia de entrada;

- Post $: T \times P \rightarrow\{0,1\}$ es la aplicación de la incidencia de salida;

- $m_{0}$ es el marcado inicial;

- $p_{0}$ es el lugar de inicio donde reside el marcado inicial; 
- $T_{0}$ es la transición de inicio del diagnosticador;

- $T_{e n d}$ es la transición que finaliza el funcionamiento del diagnosticador.

El espacio de lugares $P_{d}$ del diagnosticador es una ampliación de los lugares del modelo general refinado, un lugar $p$ de $Q_{d}$ es de la forma $P_{d}=\left(p_{i}, l_{i}\right)$ donde el lugar $p_{i}$ pertenece a los lugares observables del modelo general, $p_{i} \in P_{o}$ y la etiqueta $l_{i}$ pertenece al conjunto de las etiquetas, $l_{i} \in \triangle$, es decir de la forma $l_{i}=\{N\} \vee\left\{F_{i}\right\}$, un lugar $P_{d}$ tomará la etiqueta de funcionamiento normal o de fallo.

Un observador de $Q$ ofrece la estimación de lugar actual del sistema, después de la aparición de cada transición observable, el diagnosticador $Q_{d}$, siguiendo la dinámica de las RdP, puede entenderse conceptualmente como un observador extendido, donde se añade a cada estimación de lugar, una etiqueta del tipo de las mencionadas anteriormente, las etiquetas adjuntas indican el estado del componente, si está en modo de fallo o modo normal, los fallos se diagnostican con la validación de las etiquetas, las cuales están unidas a su respectivo lugar.

Para poder definir la evolución de la RdP diagnosticadora, se definen dos funciones fundamentales para la construcción del diagnosticador:

La función de asignación de etiqueta $L A: P_{o} \times \triangle \times T^{*} \rightarrow \triangle$, dados $p \in P_{0}$, $l \in \triangle$ y $S \in L(Q, P), L A$ asigna la etiqueta $l$ sobre la secuencia de transiciones $S$, comenzando desde $p$ y siguiendo la dinámica de $Q$, es decir, de acuerdo con:

$$
L A(p, l, S)=\left\{\begin{array}{l}
\{N\} \text { si } \forall i\left[T_{f_{i}} \notin S\right] \\
\{F\} \text { si } \forall i\left[T_{f_{i}} \in S\right]
\end{array}\right.
$$

Lo anterior significa que en la RdP diagnosticadora $Q_{d}$, asigna a cada estado su respectiva etiqueta, ésta será definida basada en el tipo de transición que sea disparada, sera $N$ si es disparada una $T_{0}$ en la secuencia $S$, o $f_{i}$ si ha sido disparada una transición de fallo del tipo $i$.

En el modelo general refinado $Q$ que representa el sistema, se integró el funcionamiento del SED's en una rama de funcionamiento normal, de la cual cuando es disparada una transición de fallo, la RdP es llevada a lugares de fallo, los cuales son interpretados como lugares sumidero, esto hace que cuando la RdP caiga allí, este modelo se bloquee. Para corregir este problema, se 
aprovecha la capacidad de concurrencia de las RdP y se define la función de Expansión de fallo.

Función de Expansión de Fallo $E F: R_{N} \times F_{i} \rightarrow R_{F}$. Donde, $R_{N}$ es la Rama de funcionamiento normal y $R_{F}$ es rama de funcionamiento de fallo. Para cada conjunto de fallo $F_{i}$ de la distribución de fallos $\Pi_{f_{i}}$ se creará una nueva rama de fallos en la RdP diagnosticadora, que cumplirá la función de supervisar los fallos individualmente. El diagnosticador $Q_{d}$ tendrá tantas ramas como fallos posea el sistema, adicionando la rama de funcionamiento normal $R_{N}, R_{Q_{d}}$ es el número total de ramas del diagnosticador:

$$
R_{Q_{d}}=R_{N}+\sum_{i=1}^{M} R_{f_{i}}
$$

\section{Construcción del Diagnosticador}

- Paso1. Definir el lugar de inicio $p_{0}$. El lugar de inicio $p_{0}$ estará compuesto por el respectivo lugar $p$ y por la etiqueta normal $N,(p,\{N\})$, y por consiguiente alberga a la marca del marcado inicial.

- Paso 2. Adicionar la transición de inicio $T_{0}$. Seguido al lugar de inicio se debe adicionar un arco unido a la transición de inicio $T_{0}$, uniendo a $p_{0}$ con la transición $T_{0}$, con la función de permitirle al operador o supervisor del sistema, el inicio de la RdP diagnosticadora.

- Paso 3. Agregar la transición final $T_{\text {end }}$. En el mismo sentido de $T_{0}$, adicionamos una transición de final de diagnóstico $T_{e n d}$, para que cuando el sistema quiera ser finalizado, se pueda tener una orden del supervisor que pueda finalizar el funcionamiento del diagnosticador. Ésta transición estará unida a $p$, por medio de un arco que va desde $T_{\text {end }}$ y finaliza en $p_{0}$.

- Paso 4. Construir la rama de funcionamiento normal $R_{N}$. Tal como se realizó en el modelo general, se adiciona la rama de funcionamiento normal, agregando lugares y transiciones que representan los estados de los diferentes componentes del sistema. En ella se podrá conocer el estado del sistema, de una manera on-line. Utilizando la función de asignación de etiqueta $L A$ la evolución de la RdP asignará su respectiva etiqueta. Dado que son lugares de funcionamiento normal, todas las etiquetas que serán asignadas en esta rama son de tipo $\Delta N$. Esta rama es fundamental para 
el operador del sistema, debido a que puede conocer el estado actual, sin importar si han ocurrido fallos o no en el proceso.

- Paso 5. Adicionar las ramas da fallo $R_{F}$. Basado en la función de expansión de fallo $E F$, para cada fallo de la partición de fallos definida anteriormente, se creará una rama de fallo. En cada rama se representará una rama normal alterna o paralela, con la variación que estando en los lugares normales, puede dispararse una transición de fallo $T_{u o}$, que ha sido reemplazada por lecturas de sensores en el modelo general refinado, o puede dispararse una transición normal $T_{N}$ que agrupa a las lecturas sensoriales $T_{S}$, esperadas por el sistema y la transición de control $T_{C}$. Cuando sea disparada una transición de fallo, conducirá al lugar de fallo $p_{f}$ y por consiguiente la función $L A$ asignará la etiqueta de fallo correspondiente al fallo $i$. Una vez la rama está en el lugar de fallo, para que ésta pueda volver al siguiente lugar (normal), las lecturas de los sensores deben ser las esperadas por el sistema unido a la transición de control. Estas ramas de fallo, funcionará de manera paralela a la normal, si aparece una lectura anormal en los sensores del sistema, es decir un fallo, la rama se quedará en ese lugar de fallo y el supervisor evidenciará el mismo, tomando las correcciones necesarias. Todas las ramas funcionan de manera independiente, lo que permite diagnosticar múltiples fallos en el mismo diagnosticador.

- Paso 6. Unión de las $T_{0}$ y $T_{\text {end }}$ a las ramas. Para finalizar la construcción de la RdPD, agregamos un arco desde la transición de inicio $T_{0}$ a cada lugar de inicio de cada rama del diagnosticador, y un arco que va desde cada lugar de inicio de cada rama a la transición de final de diagnóstico $T_{\text {end }}$.

EL diagnosticador por ser una RdP, su funcionamiento mantiene las características enunciadas en el apartado 3. Por lo tanto, siguiendo la dinámica de marcado y disparo de transiciones, en cada rama se evaluará los posibles cambios de transiciones de fallo o no esperados, y gracias a la función $L A$ el diagnosticador evoluciona en funcionamiento normal o de fallo. El diagnosticador evalúa cada fallo por separado y tiene en cuenta en sus transiciones, hasta los fallos que son causados por otros fallos, al mismo tiempo que puede detectar fallos simultáneos, sin importar el orden en que suceden los fallos. 


\subsubsection{Diagnosticabilidad}

En un sistema representado por una RdP, la diagnosticabilidad esta dada por la capacidad de poder identificar cuando el proceso cae en fallo. Por lo tanto, se dice que un sistemas es diagnosticable, si es posible detectar con un retardo finito sucesos de transiciones de fallo de cualquier tipo, utilizando el registro de transiciones observadas[77].

Diagnosticabilidad: Una RdP que representa el sistema es diagnosticable con respecto a la distribución de fallos $\Pi_{f}$ sobre la $T_{f}$ si se cumple lo siguiente:

$$
\begin{gathered}
{\left[\forall S \in \mathcal{L}\left(Q, m_{0}\right), \wedge, S \in T_{o}\right]\left(\forall i \in \Pi_{f}\right)\left(\exists n_{i} \in \mathbb{N}\right)(\forall T \in L / S)} \\
{\left[\|T\| \leq n_{i} \Rightarrow D\right]}
\end{gathered}
$$

Sea $S$ una secuencia de transiciones observables y que también pertenece al conjunto de todas las secuencias de disparo alcanzables, desde el marcado inicial $m_{0}$, y $T$ cualquier continuación suficientemente larga de $S$. La condición de diagnosticabilidad $D$ requiere que cada secuencia perteneciente al conjunto de todas las secuencias de disparo que produce las transiciones observables $S T$, debe contener una transición de fallo del conjunto $T_{f}$. Esto implica que a lo largo de cada continuación $T$ de $S$ se puede detectar la aparición de un fallo del tipo $F_{i}$ con un retardo finito, específicamente a lo sumo en $n_{i}$ transiciones del sistema después de $S$.

En un sistema se pueden presentar dos diferentes tipos de fallos, que son: fallos normales o fallos críticos, un fallo normal se puede definir como aquel que afecta el funcionamiento del sistema, pero no afecta la disponibilidad del mismo. Un fallo crítico, por lo tanto, conduce al paro total del sistema. Entonces, Se adiciona al concepto de diagnosticabilidad enunciado anteriormente, que un sistema es diagnosticable cuando permite identificar no solo fallos normales si no también, puede definir cuando un fallo crítico ha ocurrido.o-oo

Un fallo crítico $F_{C}$, es aquel fallo que pertenece a la distribución de fallos de sistema, tal que, cuando en la RdP que representa el sistema, alcanza el marcado de fallo crítico, el sistema entra en estado crítico o de fallo total.

$$
\exists F_{C} \in \prod f \rightarrow M\left(p_{f c}\right)
$$

Una RdP que representa el modelo de un sistema, es diagnosticable si en un número finito de transiciones observables, alcanza un marcado de fallo $M\left(p_{f}\right)$, 
donde el $M\left(p_{f i}\right)$, solo o en unión con otro marcado de fallo $M\left(p_{f k}\right)$, permiten identificar un fallo de orden superior o crítico.

$$
\begin{gathered}
\left(S \in \mathcal{L}\left(Q, M_{0}\right), \wedge, S \in T_{o}\right),\left(\forall i, k \in \Pi_{f}\right)[\|S\|<\propto]: \\
M\left(p_{f i}\right)+M\left(p_{f k}\right) \rightarrow M\left(p_{f c}\right)
\end{gathered}
$$

En general, el diagnosticador es una RdP implementada tomando como punto de partida el modelo general refinado del SED's, llevando a cabo una observación on-line del modelo, con objeto de realizar un diagnóstico sobre el comportamiento del sistema, también se puede utilizar para saber si existen condiciones de diagnosticabilidad en el sistema, es decir se puedan alcanzar cualquier marcado de fallo $M\left(p_{f}\right)$.

\subsection{Ejemplo de Aplicación}

En el presente documento se ha definido una metodología para realizar diagnóstico de fallos en SED's. A través de un ejemplo se pretende dar a conocer las mejoras en las dificultades que se presentan cuando se trabaja con MEF's, ahora utilizando RdP, el modelo y el diagnosticador se hacen más prácticos en su implementación. El ejemplo se realiza con un sistema de calefacción, ventilación y aire acondicionado (HVAC), este ejemplo es el mismo que fue implementado por [88][33], es importante porque de ahí se puede realizar una comparación en cuanto a construcción del modelo y diagnosticador.

\subsubsection{Ejemplo de Sistema HVAC (Heating, Ventilation and Air Condition)}

Se tiene un sistema de calefacción, ventilación y aire acondicionado, como el mostrado en la figura 4.1, el cual es usado en muchos sistemas convencionales tanto en la industria como en las residencias, está compuesto por una bomba, una válvula y un controlador, de los tres componentes se plantea fallos para la válvula y la bomba, el controlador se asume que no presenta fallos; el primer paso en esta metodología es la construcción del modelo de RdP de cada uno de los elementos que componen el sistema HVAC, ver la figura 4.2. La válvula presenta fallo en atascamiento en cerrado $A C$ y fallo en atascamiento en abierto $A A$, así mismo, la bomba presenta los fallos de: fallo de bomba en encendido $F B_{o n}$ y fallo de bomba en apagado $F B_{o f f}$, las demás transiciones que se perciben en los modelos individuales son: abrir válvula $A V$, cerrar válvu- 


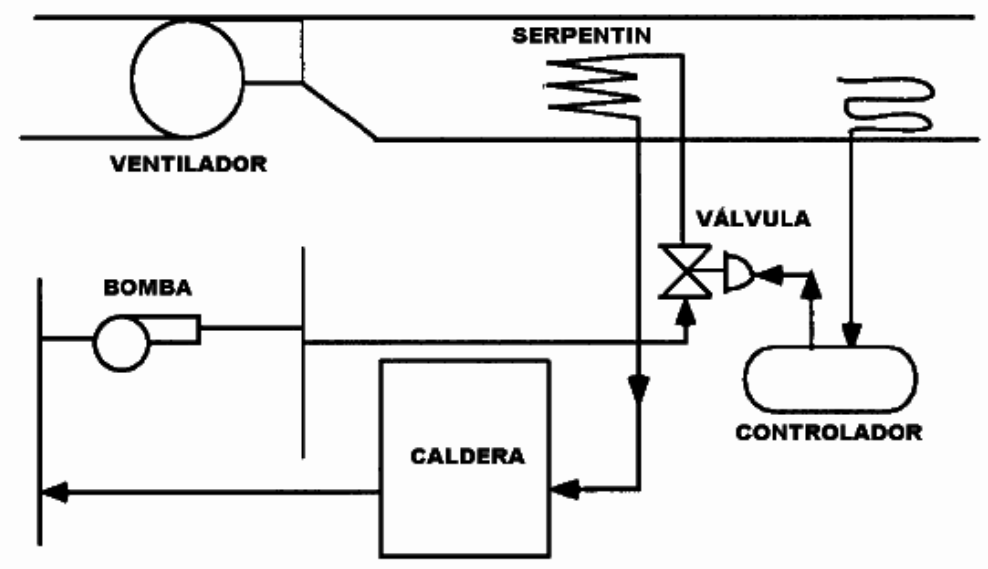

Figura 4.1: Sistema HVAC

Tabla 4.2: Lugares RdPI del Sistema Completo

\begin{tabular}{|l|}
\hline$P 1=\{C 0, B O f f, V C\}$ \\
\hline$P 2=\{C 1, B O f f, V A\}$ \\
\hline$P 3=\{C 2, B O n, V A\}$ \\
\hline$P 4=\{C 3, B O f f, V A\}$ \\
\hline
\end{tabular}

la $C V$, encender bomba $O n-B$ y apagar bomba $O f f-B$. Como transiciones no-observables del sistema se consideran las transiciones de fallo, tanto de la válvula como las de la bomba.

Se procede a realizar la operación de integración de los modelos individuales, estos se integran en una RdP general, ver figura 4.3, llevando a obtener lugares compuestos y con la dinámica impuesta por la interacción de los componentes del sistema, los nuevos lugares de la RdP que integra el sistema se pueden observar en la tabla 4.2, se puede percibir que desde cualquier lugar puede suceder los fallos de la válvula y de la bomba.

\section{Operación de Integración:}

Paso 1. Establecer el conjunto de fallos $F$, Se clasifican los fallos del sistema en conjuntos de fallo; para el ejemplo mostrado existen dos conjuntos en la partición $\Pi_{f}: \Sigma f_{1}=\{A A, A C\}, \Sigma f_{2}=\{F B o n, F B o f f\}$

- Paso 2. Definir el lugar de inicio $P_{1} . P_{1}=\left\{P_{0}^{1}, P_{0}^{2}, P_{0}^{3}\right\}$. Integra los lugares de inicio del controlador, la válvula y la bomba.

- Paso 3. Asignar un marca al lugar de inicio $P_{1}$. 

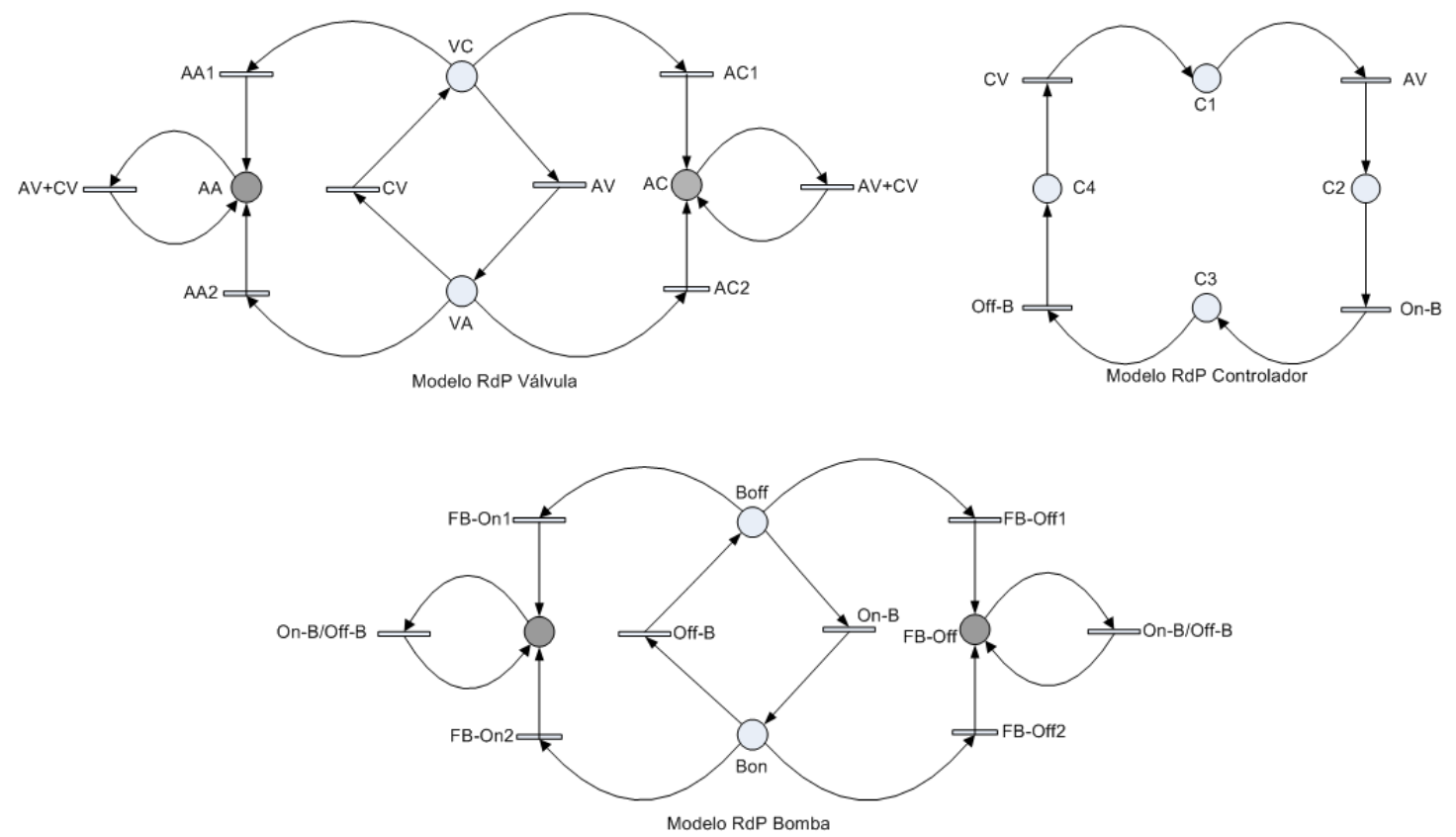

Figura 4.2: Componentes del Sistema.

- Paso 4. Construir la rama de funcionamiento normal $R_{N}$ del sistema. Desde el lugar de inicio $P_{1}$ se inicia el funcionamiento del proceso, el controlador ejecuta la transición abrir válvula $A V$, encender bomba $O n-B$, apagar bomba $O f f-B$ y por último cerrar válvula $C V$.

- Paso 5. Agregar los lugares de fallo $P_{f}$, basados en los conjuntos del paso 1.

- Paso 6. Agregar las transiciones de fallo $T_{f}$. Para cada lugar de la rama normal, se adiciona una $T_{f}$ que se va a unir con su respectivo lugar de fallo, adicionado en el paso anterior.

En el modelo general existen lugares de fallo (no observables) que no son alcanzables desde el punto de vista físico del sistema, es decir, que no podrán detectarse en esa situación en la que se encuentra el sistema o no es posible detectarlos debido a los recursos de instrumentación con que se cuenta en el mismo, entonces, para poder hacer un refinamiento se busca construir la tabla de integración de sensores, ver tabla 4.5,

\section{Refinamiento del Modelo General}




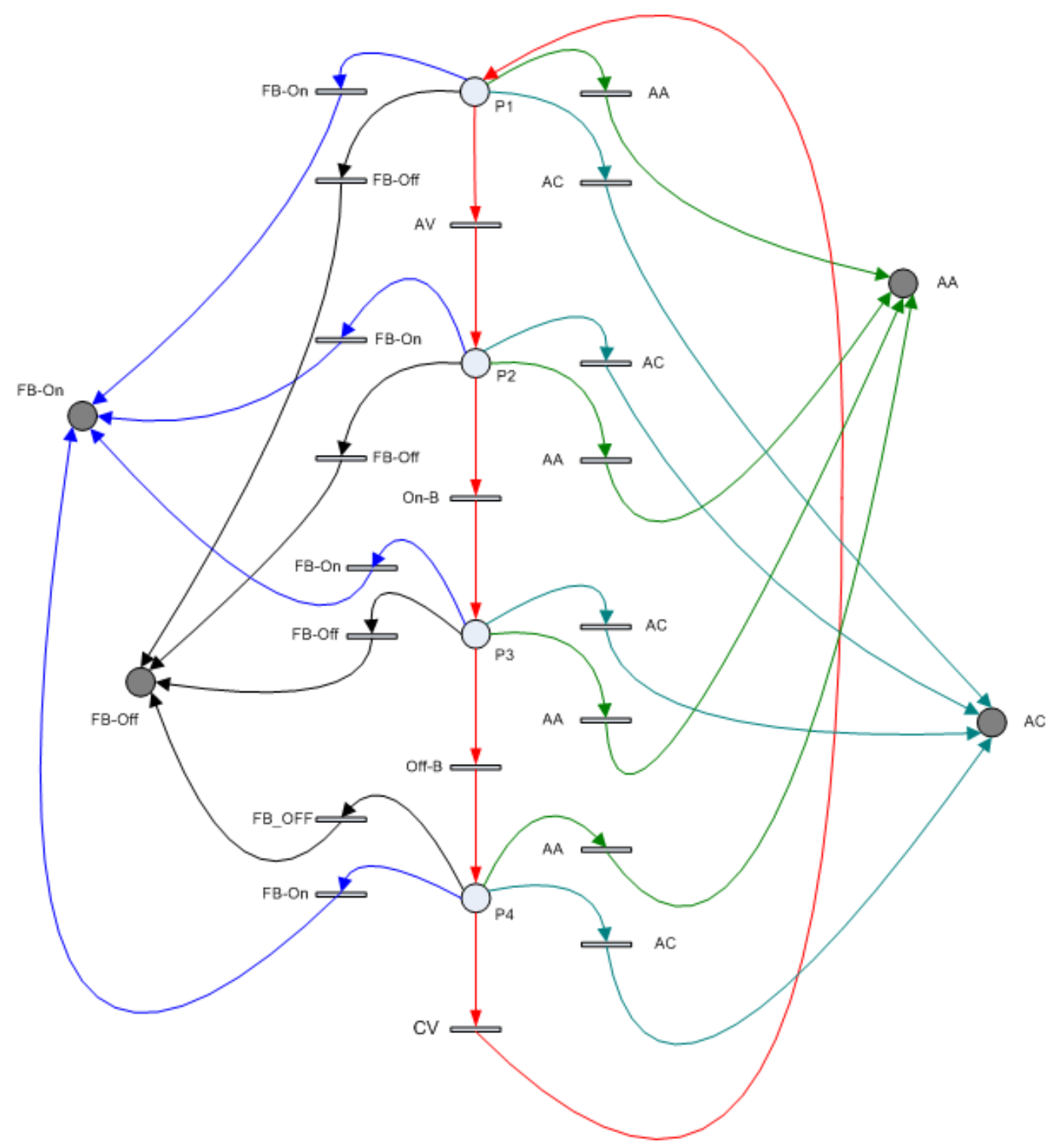

Figura 4.3: Modelo de Integración General 
Tabla 4.3: Tabla de salidas de Sensores HVAC

\begin{tabular}{|cc|}
\hline$P$ & $F$ \\
\hline \hline$N P$ & $N F$ \\
\hline$N P$ & $F$ \\
\hline$P P$ & $N F$ \\
\hline$P P$ & $F$ \\
\hline
\end{tabular}

Tabla 4.4: Salidas de la Tabla de Integración de Sensores HVAC

\begin{tabular}{|cc||c|c|c|c|}
\hline$P$ & $F$ & $P_{1}(N P, N F)$ & $P_{2}(N P, N F)$ & $P_{3}(P P, F)$ & $P_{4}(N P, N F)$ \\
\hline \hline$N P$ & $N F$ & & & & \\
\hline$N P$ & $F$ & & & & \\
\hline$P P \quad N F$ & & & & \\
\hline$P P$ & $F$ & & & & \\
\hline
\end{tabular}

- Paso 1. Identificar los sensores $S$ con que cuenta el sistema. El sistema está dotado de dos sensores: flujo $F$ y presión $P$, estos dan como señales de salida discreta flujo $F$, no flujo $N F$, presión positiva $P P$, no presión $N P$, respectivamente.

- Paso 2. Construir el conjunto discreto de posibles salidas de los sensores que componen el sistema. $N=2$. Las combinación representados en la tabla 4.3, se convierten en las entradas de la tabla de integración de sensores.

- Paso 3. Definir las salidas de la tabla de integración. Cada lugar normal de la RdP mantiene lecturas de los sensores, que describen su funcionamiento normal y son un referente de elegir cuando la RdP está en estado normal, Fallo o no determinado, estas lecturas normales son las salidas de la tabla de integración de sensores, ver tabla 4.4.

- Paso 4. Construir la tabla de integración de sensores $h_{j}=\widetilde{P} \rightarrow \widetilde{Y}_{j}$. Desde el estado normal de cada lugar y teniendo en cuenta el estado de las transiciones de control, se comparan las lecturas de los sensores con todas las combinaciones de las lecturas de sensores, si es igual, el estado es normal, si hay diferencia, la RdP puede conducir a un lugar de fallo o no determinado $X$, por ejemplo, estando la RdP en el lugar $P_{1}(N P, N F)$, las lecturas de los sensores son $(P P, N F)$ y las transiciones $A V$ y $O n-B$ 
CAPÍTULO 4. CONSTRUCCIÓN DEL MODELO Y DIAGNOSTICADOR USANDO RDP PARA FD DE SED'S

Tabla 4.5: Tabla de Integración de Sensores HVAC

\begin{tabular}{|cc||c|c|c|c|}
\hline$P$ & $F$ & $P_{1}(N P, N F)$ & $P_{2}(N P, N F)$ & $P_{3}(P P, F)$ & $P_{4}(N P, N F)$ \\
\hline \hline$N P$ & $N F$ & $N$ & $N$ & $F B_{O f f}-A C$ & $N$ \\
\hline$N P$ & $F$ & $X$ & $X$ & $F B_{O f f}$ & $X$ \\
\hline$P P$ & $N F$ & $F B_{O n}$ & $F B_{O n}-A C$ & $A C$ & $F B_{O n}-A C$ \\
\hline$P P$ & $F$ & $F B_{O n}-A A$ & $F B_{O n}$ & $N$ & $F B_{O n}$ \\
\hline
\end{tabular}

están activas, podemos deducir que ha ocurrido un fallo $F$ Bon. Si el sistema recibe las lecturas de $(N P, F)$ con las transiciones de $A V$ y $O f f-B$ activas, aunque hay un cambio de lectura de $N F$ a $F$, no se puede deducir ninguna información basado en que la bomba está apagada y no podemos tener flujo, las demás combinaciones se pueden ver la tabla4.5

- Paso 5. Eliminar los lugares de fallo no alcanzables. Basado en la tabla de integración de sensores se elimina de la RdP los lugares y transiciones no alcanzables, y se remplazan las transiciones no-observables por las lecturas sensoriales donde corresponda. Por ejemplo, estando en el lugar $P_{1}$, sustituimos la transición de fallo de $A A$ por $P P \cdot F$, es decir que podemos conducir la RdP al lugar de fallo de $A A$ si las lecturas de los sensores nos indican que hay presión $P P$ y hay flujo $F$. El refinamiento total de la $\mathrm{RdP}$ general se puede ver en la figura 4.4 .

De esta intersección de lecturas de los sensores, se manifiestan las transiciones de fallo que son viables o detectables, así como también, transiciones de fallo que son dependientes de otros fallos, por lo tanto, con este procedimiento se puede detectar no solo fallos simultáneos, si no también fallos dependientes, sin importar el orden de los sucesos de los fallos.

Con un modelo del sistema bien definido y sin transiciones no observables, se procede a la construcción del diagnosticador, el diagnosticador es una RdPD. Basado en que tenemos dos grupos de fallos, fallos de la válvula $(A A, A C)$ y fallos de la bomba ( $F B$ on, $F B$ of $f$ ), en el diagnosticador nos resultan dos etiquetas de fallo $\Delta F=\left\{F_{1}, F_{2}\right\}, F_{1}\left(\Sigma f_{1}=\{A A, A C\}\right)$ y $F_{2}\left(\Sigma f_{2}=\{F B o n, F B o f f\}\right)$, con estos datos y basado en la funciones de: asignación de etiquetas y expansión de fallos, se construye el diagnosticador. En el diagnosticador se presentan dos ramas, asignadas al fallo de válvula y otra al fallo de bomba, 


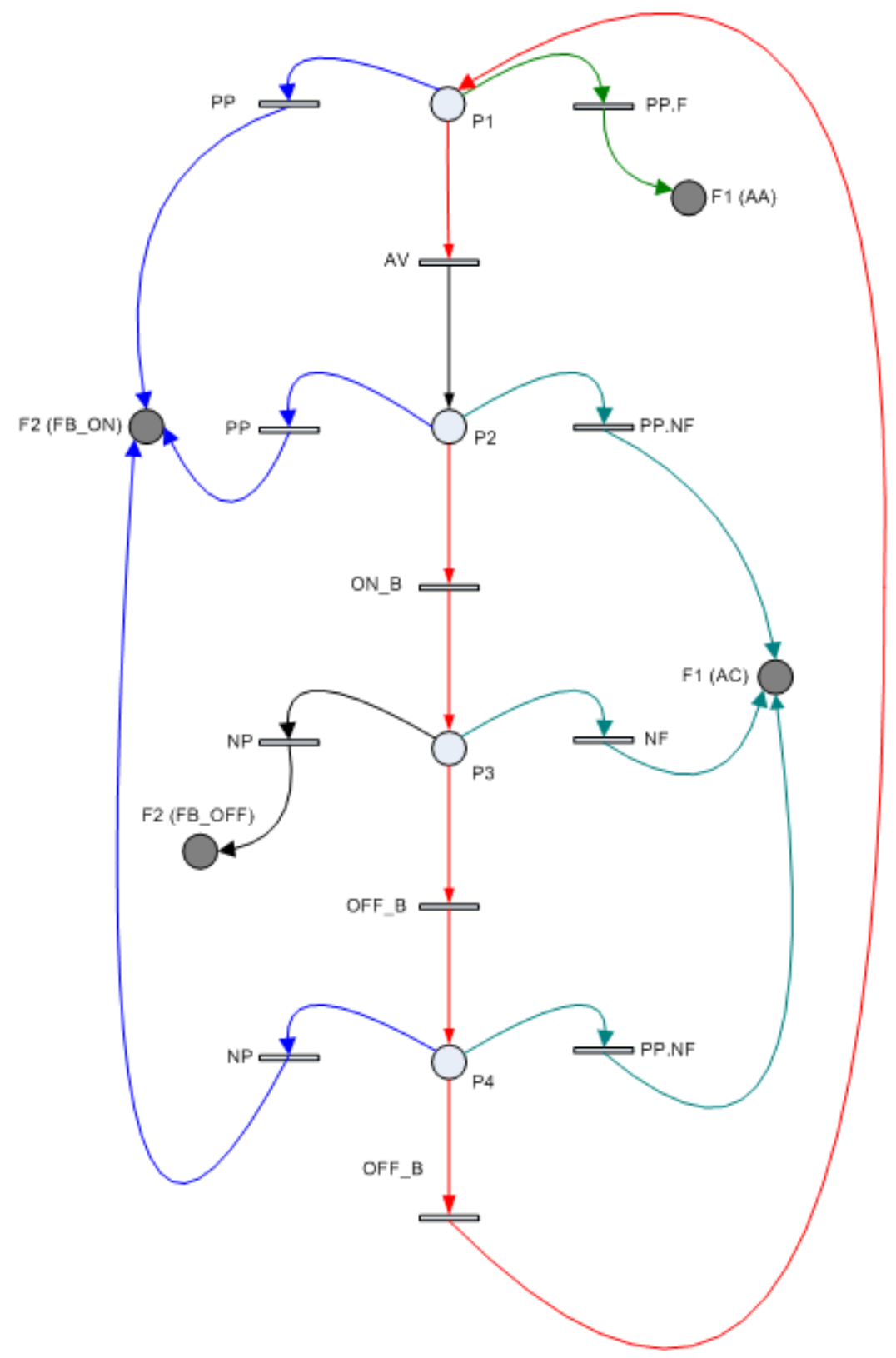

Figura 4.4: Modelo de Integración Refinado 


\section{Construcción del Diagnosticador}

- Paso1. Definir el lugar de inicio $p_{0}$.

- Paso 2. Adicionar la transición de inicio $T_{0}$.

- Paso 3. Agregar la transición final $T_{\text {end }}$.

- Paso 4. Construir la rama de funcionamiento normal $R_{N}$. Tal como se realizó en el modelo general. Dado que son lugares de funcionamiento normal, todas las etiquetas que serán asignadas en esta rama son de tipo $\Delta N$.

- Paso 5. Adicionar las ramas da fallo $R_{F}$. Tenemos, Rama de fallo de Bomba y Rama de Fallo de Válvula, La rama esta compuesta de los mismo lugares normales de la rama normal y basado en las transiciones del controlador $T_{C}$ y las transiciones de los sensores $T_{S}$, puede caer en fallo o continuar su funcionamiento normal. Por ejemplo, Una vez activada $T_{0}$, tenemos una marca en el lugar $P 1$, si las lecturas de los sensores cambian de $N P$ a $P P$, denota que la transición de $F B o n$ es disparada y la rama cae en fallo. pero si las lecturas se mantienen normales $(N P, N F)$ y se dispara la transición de $A V$, la rama de fallo de bomba sigue su funcionamiento normal. El mismo esquema de funcionamiento se mantiene para la rama de fallo de válvula.

- Paso 6. Unión de las $T_{0}$ y $T_{\text {end }}$ a las ramas.

El diagnosticador evolucionará normalmente y las dos ramas estarán ejecutándose simultáneamente en funcionamiento normal, ver figura 4.5, por lo tanto, las etiquetas asignadas son normales $N$, en el momento en que suceda un fallo de cualquier tipo, la rama en la cual entra en fallo es asignada la respectiva etiqueta de fallo $F_{1} \circ F_{2}$, esa rama queda bloqueada indicando al supervisor que ha ocurrido un fallo, si dado el caso de que el fallo sea recuperado, es decir las lecturas de sensores vuelven a su estado normal, el proceso podrá seguir evolucionando normalmente, así mismo, en este diagnosticador puede ocurrir los dos fallos a la vez y ser diagnosticados sin importar el orden de aparición. 


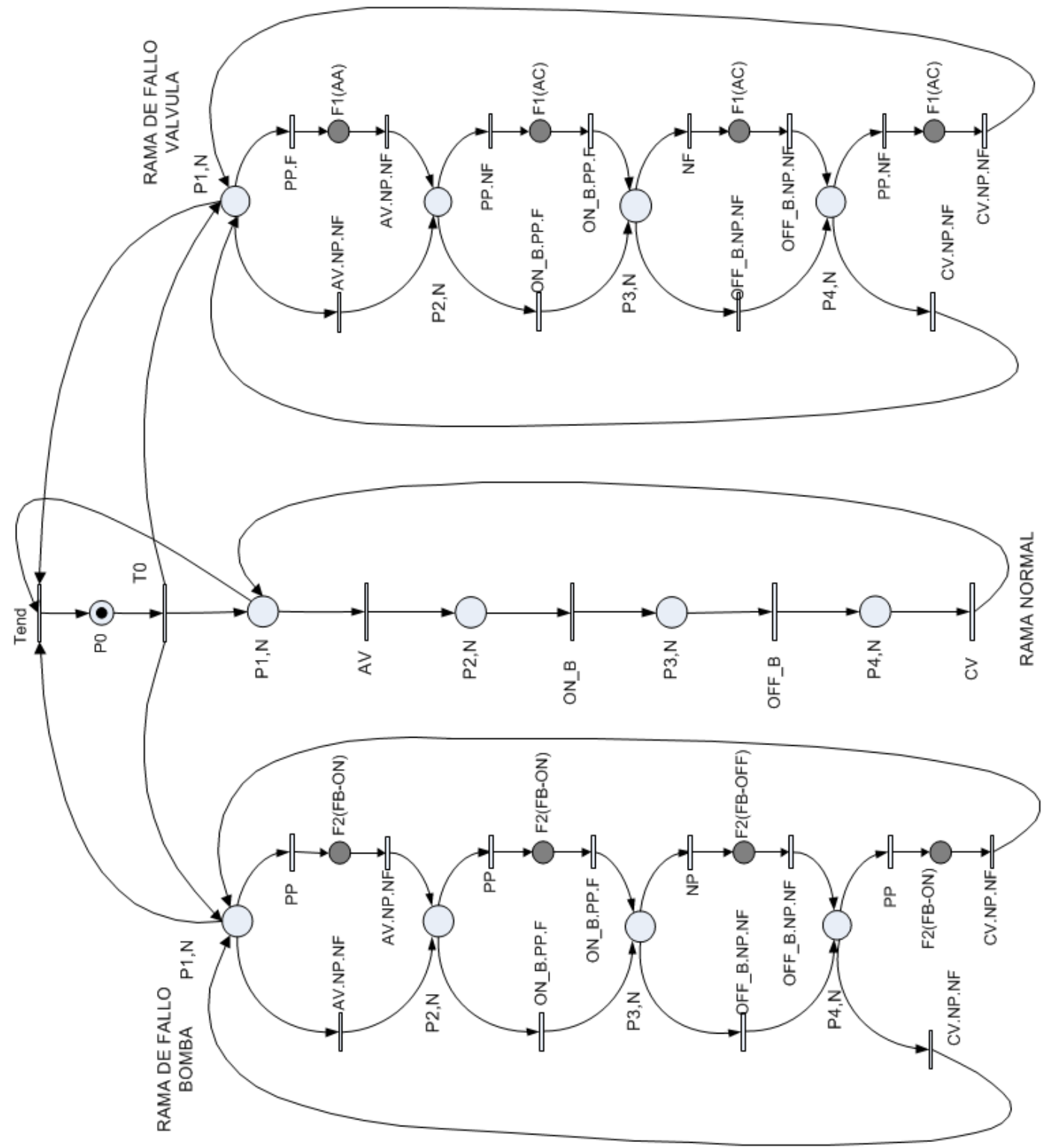

Figura 4.5: Diagnosticador 


\subsection{Conclusión}

Anteriormente se han dado suficientes elementos para construir el modelo del SED's, así como también el diagnosticador, por lo tanto, a manera de resumen, se presenta a continuación el algoritmo que describe los pasos fundamentales para implementar el modelo y diagnosticador de un proceso:

1. Construir cada uno de los modelos de RdP de los componentes del SED's. $R_{i}=\left(P_{i}, T_{i}\right.$, Pre, Post,$\left.m_{0}\right)$

2. Clasificar los conjuntos de fallo que se puede dar de acuerdo a los elementos del sistema. $\Pi_{f}$

3. Construir el modelo de RdP de integración de los componentes del proceso.

$$
\widetilde{Q}=(\widetilde{P}, \widetilde{T}, \widetilde{P r e}, \widetilde{\text { Post }}) . \widetilde{P}=\bigcup_{i} P_{i} \quad y \quad \widetilde{T}=\bigcup_{i} T_{i} .
$$

4. Realizar la tabla de integración de sensores. $h_{j}=\widetilde{P} \rightarrow \widetilde{Y}_{j}$.

5. Basado en la tabla de integración de sensores, hacer los cambios necesarios de transiciones no observables a observables y descartar las transiciones no alcanzables. $\forall p_{i}: p_{i} \in P \rightarrow M\left(p_{i}\right) \notin R\left(Q, m_{0}\right)$.

6. Refinar el modelo de integración del sistema $\widetilde{Q}=(\widetilde{P}, \widetilde{T}, \widetilde{\text { Pre }}, \widetilde{\text { Post }})$ a $Q=$ (P, T, Pre, Post).

7. Definir las etiquetas de fallo. $\triangle f=\left\{F_{1}, F_{2}, \ldots, F_{m}\right\},\left|\Pi_{f}\right|=m$.

8. De acuerdo a las funciones de $A L$ y $E F$ construir el diagnosticador $Q_{d}$, donde se desarrolle una rama para cada fallo, $R_{Q_{d}}=R_{N}+\sum_{i=1}^{M} R_{f_{i}}$.

Con el algoritmo de implementación del modelo y diagnosticador presentado, se confirma que la técnica desarrollada es un herramienta de diagnostico de fallos sistemática, la cual tiene gran proyección hacia futuros trabajos del área. Después de haber comparado el algoritmo con trabajos previos en diagnóstico de fallo de SED's, podemos manifestar las ventajas o resultados positivos que se dan en este algoritmo de diagnóstico de fallos, las ventajas más representativas de ésta metodología se describen a continuación:

1. Fácil construcción e implementación en cualquier proceso de SED's. 
CAPÍTULO 4. CONSTRUCCIÓN DEL MODELO Y DIAGNOSTICADOR USANDO RDP PARA FD DE SED'S

2. Detección y aislamiento de fallos simultáneos.

3. Detección y aislamiento de fallos que son dependientes de otros fallos.

4. Detección y aislamiento de fallos sin importar el orden de como suceden los fallos en el proceso.

5. Cumple funciones de supervisor, informa el estado actual en que se encuentra el sistema.

6. La RdP diagnosticadora nunca se bloquea, es decir, no presenta estados sumidero.

7. La estructura diseñada esta pensada para usarse en el diagnóstico de fallos intermitentes.

Con el algoritmo de diagnostico de fallos implementado, se mejoran en gran medida la mayoría de los problemas que presentaban las técnicas desarrolladas mediante MEF's, entre las más destacada, la explosión combinacional. 


\section{Capítulo 5}

\section{Adición de la Dinámica Temporal al Diagnosticador}

En los procesos industriales, los fallos en los componentes son eventos que se pueden diagnosticar, tal como se ha tratado en los capítulos anteriores. Hasta el momento se ha visto que los fallos son detectados cuando ocurren definitivamente, pero en la realidad de los procesos, existen fallos que pueden ser fallos definitivos o fallos progresivos, es decir, éstos en la mayoría de los casos, tienden a pasar de estado normal a fallo y posteriormente de estado de fallo a normal, esta dinámica se vuelve repetitiva con una duración de tiempo $t$ en estado de fallo, que inicia en valores cercanos a cero y después se va ampliando hasta alcanzar un valor de tiempo infinito (fallo definitivo), por lo tanto, los dispositivos pasan de un estado de fallo intermitente (Fl's) a fallo definitivo. Así, se puede concluir que un fallo intermitente es un preámbulo a un fallo definitivo.

Para cualquier sistema, es de gran importancia detectar un posible fallo, antes de que éste ocurra, y no esperar a un fallo definitivo, que pueda causar el paro de una planta, donde se desaten grandes pérdidas económicas o en algunas ocasiones humanas. Por lo tanto, el diagnóstico de fallos intermitentes es un gran aporte en lo que respecta al mantenimiento preventivo y predictivo, donde se deduce el estado de los componentes del proceso, antes de su fallo definitivo, además, se establece un reemplazo del componente de una manera programada, evitando que todo un proceso se pare y genere perdidas incalcu- 


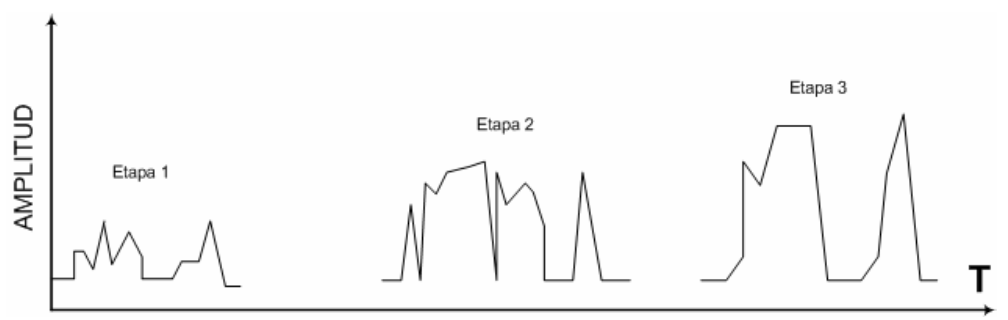

Figura 5.1: Evolución de los Fl's a lo Largo de la Vida del Componente

lables.

La evolución de los fallos intermitentes fue comprobada por [18], ver figura 5.1, en esta gráfica se puede apreciar como los fallos van progresivamente sufriendo una degradación, donde a través de etapas se ve que inicialmente es prácticamente imposible detectar los fallos, debido a sus efectos y su corta duración. Pero a medida que se avanza en el uso de los mismos, la amplitud tanto como la duración incrementan dando fallos aleatorios en el sistema. Normalmente, los fallos no son detectados hasta que se llega hasta la tercera etapa o cuando se convierten en definitivos.

En el presente capítulo se pretende incorporar el diagnóstico de Fallos Intermitentes en los SED's al Modelo y diagnosticador basados en RdP, dados en el capítulo 4, también se busca anexar los Fl's en la diagnosticabilidad del sistema y aplicar estos nuevos conceptos en el ejemplo del proceso de envasado de líquidos utilizado como ejemplo de referencia en el capitulo 4.5.

El concepto de Fallo intermitente parte de los estudios desarrollados por [16][52][69], donde se establece que el problema básico de diagnóstico de Fl's es: "A partir de una secuencia de entradas $U$ salidas $Y$, obtenidas a partir de los sensores instalados en el proceso, determinar la presencia de un fallo $f$ (del conjunto de posibles fallos $F$ ), así como su posible recuperación $r f$ ". En esta definición se puede percibir que se diagnostican eventos que llevan el sistema a un estado de fallo con su respectiva recuperación. Sin embargo, según [18] argumenta que los Fl's tienen una dinámica propia que obliga a que la definición sea ampliada a que se debe detectar, tanto fallos y recuperación, como su dinámica temporal. En esta definición se puede detectar el efecto y la evolución del Fl's, aunque no deja de ser un problema complejo la misma detección de su dinámica. Por ello, basado en el desarrollo planteado por [16], abordaremos a 


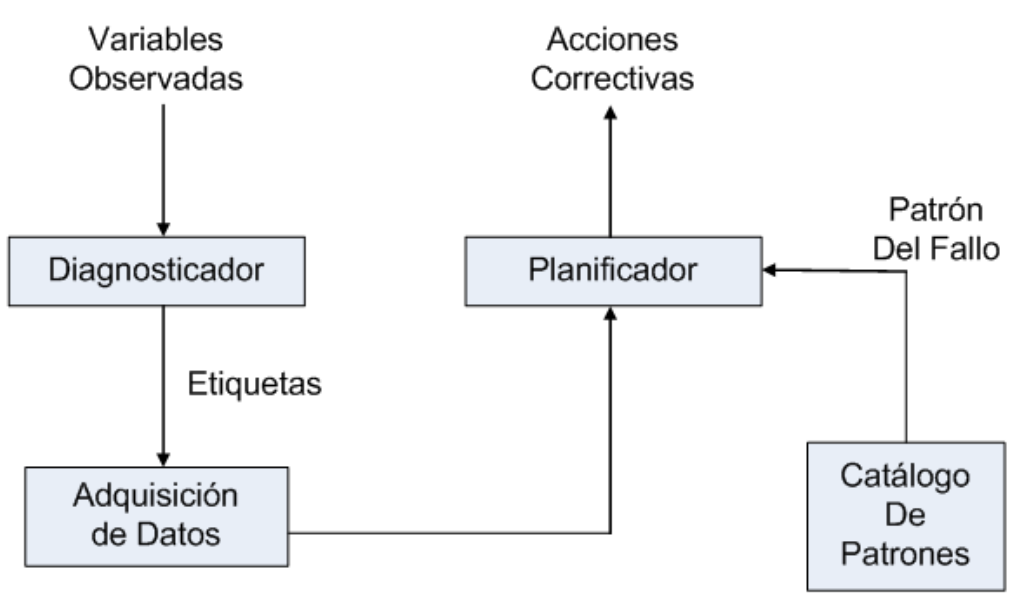

Figura 5.2: Arquitectura de Diagnóstico de Fl's

continuación el diagnóstico de Fl's junto a su dinámica temporal, partiendo de su arquitectura de diagnóstico de Fl's, ver figura 5.2.

\subsection{Modelo y Diagnosticador para Fl's}

Partiendo de lo planteado en el capítulo 4, para realizar diagnóstico de Fl's, debemos plantear en el modelo de RdP de cada uno de los elementos que componen el sistema, se incluya la recuperación de los fallos, donde el modelo de $\mathrm{RdP}$ de cada elemento quedará de la forma: $R_{i}=\left(P_{i}, T_{i}\right.$, Pre, Post, $\left.m_{0}\right)$, donde la única variación dada es que al conjunto de las transiciones $T$, deben ser agregadas las nuevas transiciones de recuperación $T_{R}$. Entonces tenemos que: $T=T_{o} \cup T_{u o}, T_{u o}=T_{f} \cup T_{R}$. Esta nuevas transiciones de recuperación de fallo, se usarán únicamente en el diagnosticador, ya que es realmente donde prestan importancia.

Siguiendo la misma dinámica planteada en los procesos de construcción realizados en el capítulo 4, se procede a realizar el modelo general de integración del sistema $\widetilde{Q}=\left(\widetilde{P}, \widetilde{T}, \widetilde{P r e}, \widetilde{\text { Post }}, m_{0}\right)$. Una vez el modelo general es construido, se construye la tabla de integración de sensores, basados en los sensores con que cuenta el sistema. Estos procesos de construcción son idénticos a los que se construyeron en el capítulo 4. Una vez la tabla de integración de sensores es construida, se hace la refinación del modelo general $Q=\left(P, T\right.$, Pre, Post,$\left.m_{0}\right)$. Este modelo solo queda compuesto de transiciones 


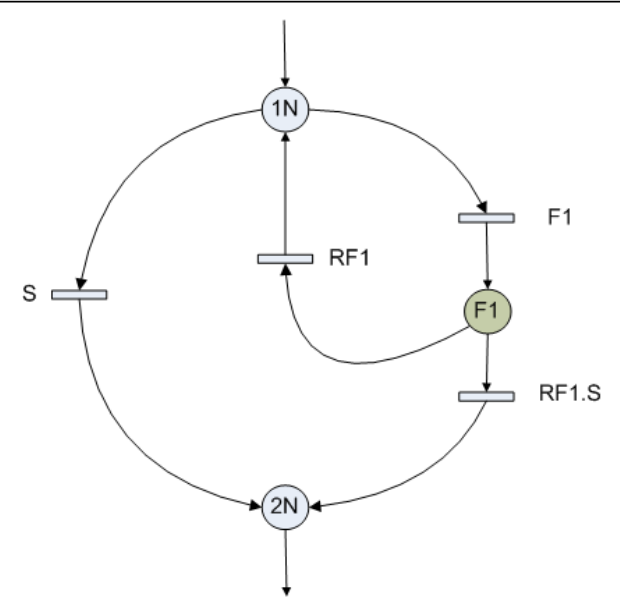

Figura 5.3: Variación de Diagnosticador para Fl's

observables, dadas por las transiciones de ordenes de control $T_{C}$ y la combinación de las transiciones dadas por las lecturas sensoriales $T_{S}, T=T_{C} \cup T_{S}$, Las $T_{S}$ serán las encargadas de representar a las transiciones de recuperación $T_{R}$, que se mantienen como lecturas sensoriales.

Después de incorporar en el modelo de RdP que representa el sistema, las variaciones necesarias para el diagnóstico de Fl's se realizan directamente en el diagnosticador, quien es realmente el que realiza la observación on-line del sistema. Puede existir dos tipos de situaciones en lo que respecta a las recuperación de la transición de fallo, que haya recuperación y el diagnosticador tenga que volver al lugar anterior o por sus condiciones de evolución, el diagnosticador tenga que pasar al lugar siguiente. Esto se puede diferenciar de la siguiente manera: a) El diagnosticador vuelve al lugar anterior: Aquí solo tendrá en cuenta las lecturas de los sensores $T_{R}=T_{S}$. b) El diagnosticador pasa al lugar siguiente: Se tienen en cuenta las transiciones de los sensores, junto con las transiciones de control, $T_{R}=T_{C} \bullet T_{S}$. Por lo tanto, a cada uno de los lugares de fallo de las ramas de fallo, con respecto a lo planteado en el capítulo 4, se añadirá una transición de recuperación $T_{R}$ que devuelva el diagnosticador al estado normal.

$$
\left(\forall p_{f_{i}} \in P_{F}\right)\left(\exists T_{R_{i}} \in T_{R}: T_{R}=T_{S}\right)\left(p_{f_{i}} \times T_{R_{i}} \rightarrow P_{N(i-1)}\right)
$$

$i=1, \ldots, M, M$ es el número de fallos que posee el sistema dado en la partición $\Pi_{f}$. Esta nueva variación en el diagnosticador la puede observar en la figura 5.3. 


\subsection{Diagnóstico Temporal de Fl's}

En los trabajos previos de diagnóstico de Fl's dados por [18], aplicados a SED's utilizando MEF's, planteó que para conseguir un diagnóstico de Fl's completo, se realiza correctamente cuando se detecta los eventos de fallo, sus recuperaciones y su dinámica temporal. En los estudios realizados, implementó varias técnicas que conducían a conseguir indicadores temporales, en pro de tomar decisiones en el mantenimiento preventivo de los dispositivos del proceso, realizándolo a través de la comparación del estado del componente con un patrón de fallos realizado previamente. Las técnicas utilizadas por [18] son: el diagnóstico acotado de la dinámica del Fl's con métodos estadísticos y el diagnóstico acotado de la dinámica del Fl's con la densidad temporal. Basado en estos estudios, formula los llamados Diagnosticadores Extendidos (DE), los cuales permiten incorporar en los estados del diagnosticador una variable de tiempo y una variable de conteo, que sirven de referencia en el planteamiento dado en el presente documento.

Según lo presentado anteriormente para diagnóstico de Fl's con RdP, ya se ha incluido las transiciones de fallo y su recuperación, ahora procedemos a incluir la dinámica temporal en el diagnosticador. La tarea adicional comprende dos anexos, en primer lugar, para cada uno de los lugares de fallo, se debe agregar una variable de tiempo $j$ que se inicia en el momento que el lugar es marcado, es decir, inicia cuando ocurra un fallo, y finaliza cuando la marca del lugar desaparece gracias a la recuperación del fallo o el disparo de la transición que lleva la rama al siguiente lugar normal. Como $j$ almacena un tiempo, este tiempo será sumado a los demás tiempos en que entra en fallo la rama, en las diferentes transiciones de fallo que puede ocurrir en el proceso. $\forall p_{f_{i}} \in P_{F}, \exists j \rightarrow$ $p_{f_{i}}=\left(M\left(p_{i}\right), j_{i}\right)$, donde $i=1, \ldots, M, M$ es el número de fallos del sistema. Para cada rama de fallo del sistema, existe un lugar de conteo temporal $p_{C}$, donde cada vez que se habilite una transición de fallo de la rama, a su vez, estas transiciones le asignarán una marca a este lugar de conteo, definiendo que por cada marca que posea el lugar, significa que el fallo ha sido $n$ veces disparado. También en este lugar de conteo temporal $p_{C}$, se realizará la sumatoria de los tiempos que suceden en cada unos los lugares de fallo, almacenados en la variable $j$. Esto muestra entonces, que desde cada lugar de fallo de la rama, debe salir un arco que finaliza en el lugar de conteo temporal de la rama $p_{C}$. 


$$
\begin{gathered}
\forall R_{F} \in R, \quad \exists p_{C}: t_{f_{i}} \rightarrow p_{C}, \wedge, J=\sum_{i=1}^{M} j_{i}, \\
m\left(p_{C}\right)=\sum_{i=1}^{M} m\left(p_{f_{i}}\right), i=1, \ldots, M
\end{gathered}
$$

En resumen, en el lugar de conteo, se realiza la suma de los tiempos de fallo en que dura un fallo intermitente y las veces que sucede un fallo, con estos datos de tiempo y número de veces que ha fallado un dispositivo, se puede llevar a cabo la determinación del patrón de referencia de fallo, fundamental en los análisis del mantenimiento preventivo de los componentes. Cuando el supervisor del sistema decide finalizar el uso diagnosticador, debido a que un elemento debe ser reemplazado o reparado, se debe resetear las ramas de fallo del diagnosticador, es decir poner el contador de fallos a cero y resetear la variable de tiempo $J$, para ello se agrega un arco que va desde el lugar de conteo $p_{C}$ a la transición de final de diagnostico $T_{\text {end }}$, y desde la transición de final de diagnóstico a otro arco que no tiene lugar de llegada, el peso de este arco es de $n$ marcas del $P_{C}$, haciendo que el lugar de conteo quede sin marcas y que la variable de tiempo total $J$ sea reiniciada en cero. Por ende, se podría iniciar una nueva etapa de diagnóstico de Fl's. En conclusión, el lugar de conteo realiza las operaciones de conteo de fallos y la suma de tiempos de fallo del componente diagnosticado. Para ver la nueva configuración de la rama de fallo del diagnosticador ver la figura 5.4.

Basados en que al incluir los Fl's en la herramienta de FD solo se hacen variaciones en el diagnosticador, a continuación se hacen las modificaciones pertinentes al algoritmo de construcción de diagnosticador:

\section{Construcción del Diagnosticador}

- Paso1. Definir el lugar de inicio $p_{0}$. El lugar de inicio $p$ estará compuesto por el respectivo lugar $p$ y por la etiqueta normal $N,(p,\{N\})$, y por consiguiente alberga a la marca del marcado inicial.

- Paso 2. Adicionar la transición de inicio $T_{0}$. Seguido al lugar de inicio se debe adicionar un arco unido a la transición de inicio $T_{0}$, uniendo a $p_{0}$ con la transición $T_{0}$, con la función de permitirle al operador o supervisor del sistema, el inicio de la RdP diagnosticadora.

- Paso 3. Agregar la transición final $T_{\text {end }}$. En el mismo sentido de $T_{0}$, adicionamos una transición de final de diagnóstico $T_{\text {end }}$, para que cuando el 


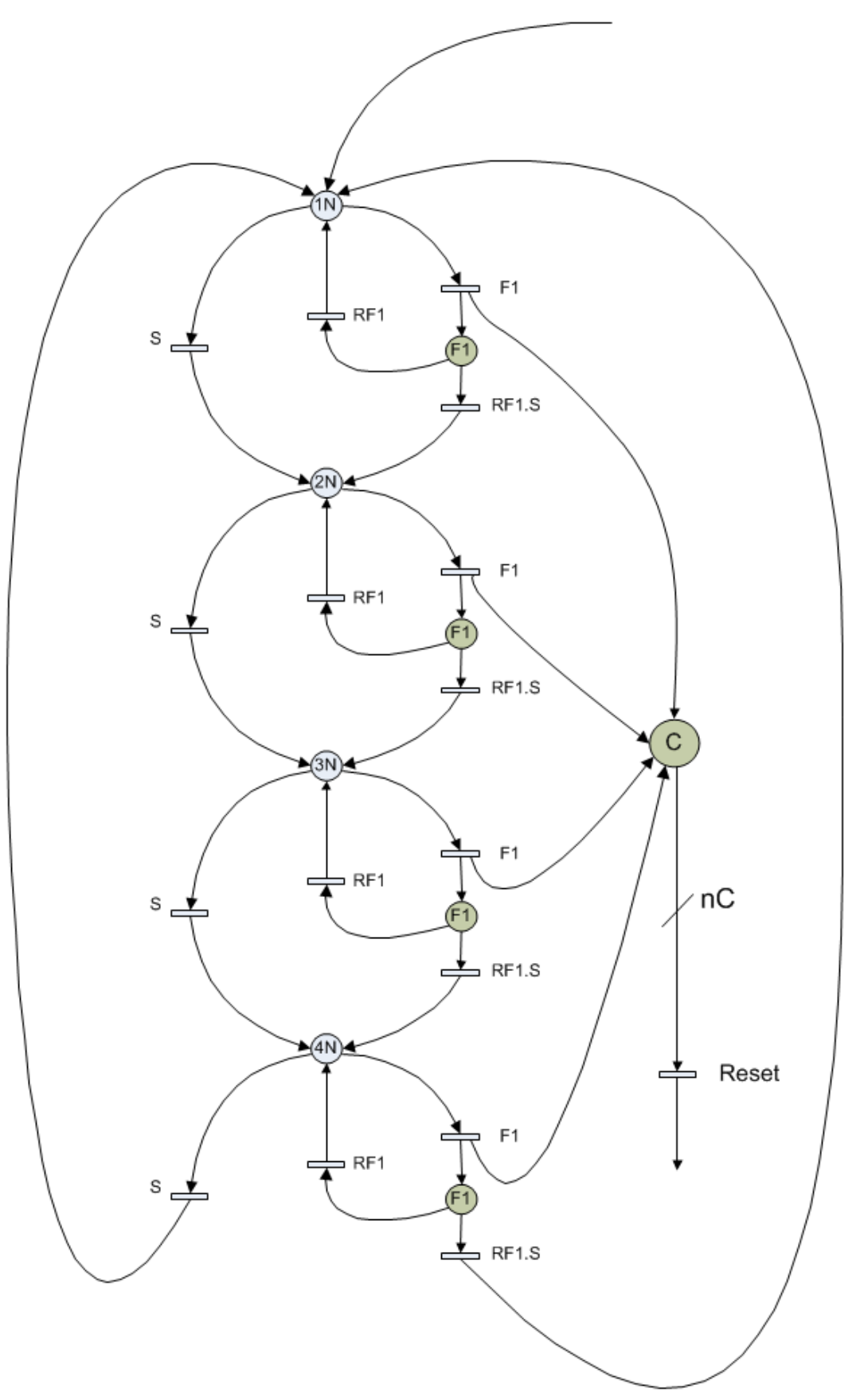

Figura 5.4: Rama para Diagnóstico de Fl's 
sistema quiera ser finalizado, se pueda tener un orden del supervisor que pueda finalizar el funcionamiento del diagnosticador. Esta transición estará unida a $p_{0}$, por medio de un arco que va desde $T_{\text {end }}$ y finaliza en $p_{0}$.

- Paso 4. Construir la rama de funcionamiento normal $R_{N}$. Tal como se realizó en el modelo general, se adiciona la rama de funcionamiento normal, agregando lugares y transiciones que representan los estados de los diferentes componentes del sistema. En ella se podrá conocer el estado del sistema, de una manera on-line. Utilizando la función de asignación de etiqueta $L A$ la evolución de la $\mathrm{RdP}$ asignará su respectiva etiqueta. Dado que son lugares de funcionamiento normal, todas las etiquetas que serán asignadas en esta rama son de tipo $\Delta N$. Esta rama es fundamental para el operador del sistema, debido a que puede conocer el estado actual, sin importar si han ocurrido fallos o no en el proceso.

- Paso 5. Adicionar las ramas da fallo $R_{F}$. Basado en la función de expansión de fallo $E F$, para cada fallo de la partición de fallos definida anteriormente, se creará una rama de fallo. En cada rama se representará una rama normal alterna o paralela, con la variación que estando en los lugares normales, puede dispararse una transición de fallo $T_{u o}$, que ha sido reemplazada por lecturas de sensores en el modelo general refinado, o puede dispararse una transición normal $T_{N}$ que agrupa a las lecturas sensoriales $T_{S}$, esperadas por el sistema y la transición de control $T_{C}$.

- Cuando sea disparada una transición de fallo, conducirá al lugar de fallo $P_{f}$ y por consiguiente la función $L A$ asignará la etiqueta de fallo correspondiente al fallo $i$. Una vez la rama está en el lugar de fallo, existe la posibilidad de que la rama regrese a su estado normal anterior, es decir la transición de recuperación $T_{R}$ sea activada o pueda volver al siguiente lugar (normal), es decir, las lecturas de los sensores deben ser las esperadas por el sistema, unido a la transición de control.

Estas ramas de fallo, funcionará de manera paralela a la normal, si aparece una lectura anormal en los sensores del sistema, es decir un fallo, la rama se quedará en ese lugar de fallo y el supervisor evidenciará el mismo, tomando las correcciones necesarias. Todas las ramas funcionan de 
manera independiente, lo que me permite diagnosticar múltiples fallos en el mismo diagnosticador.

- Paso 6. Crear para cada lugar de fallo la variable de tiempo $j$. Esta variable de tiempo es iniciada con el marcado del lugar de fallo y finalizará cuando cualquier transición de salida del lugar de fallo mencionado, es disparado.

- Paso 7. Crear para cada rama de fallo, el lugar de conteo temporal $P_{C}$. Desde cada transición de fallo, se crea un arco para que sea unido a $P_{C}$, el cual irá acumulando una marca por cada fallo en la rama.

- Paso 8. Crear la Transición de Reinicio $T_{r e s e t}$ de cada rama de fallo. Esta transición esta conectada por un arco desde $P_{C}$ con un peso de $n$ marcas del lugar de conteo $P_{C}$, y de salida tiene un arco sin lugar de destino, el cual cumplirá la función de vaciar el lugar $P_{C}$ cuando el diagnosticador es finalizado.

- Paso 9. Unión de las $T_{0}$ y $T_{\text {end }}$ a las ramas. Para finalizar la construcción de la RdPD, agregamos un arco desde la transición de inicio $T_{0}$ a cada lugar de inicio de cada rama del diagnosticador, y un arco que va desde cada lugar de inicio de cada rama a la transición de final de diagnóstico $T_{\text {end }}$.

\section{Diagnosticabilidad.}

La diagnosticabilidad de un sistema esta dada por la capacidad del diagnosticador de poder identificar cuando el proceso cae en fallo. Ahora que se hace una extensión del diagnosticador para fallos intermitentes, se puede afirmar que un sistema es diagnosticable cuando permite identificar fallos, teniendo en cuenta las intermitencias de los mismos, para determinar la vida útil del dispositivo y la aparición de un fallo superior o crítico.

Una RdP que representa el modelo de un sistema, es diagnosticable si en un número finito de transiciones observables, alcanza un marcado de fallo $M\left(p_{f}\right)$, donde el $M\left(p_{f i}\right)$, solo o en unión con otro marcado de fallo $M\left(p_{f k}\right)$, permiten identificar un fallo de orden superior o crítico. Además, pueda establecer el reemplazo de los componentes del sistema.

Patrón de Vida Útil (PU): Son los parámetros del componente en diagnóstico, que sirven para decidir su reemplazo o reparación $\mathcal{R}$. 
Diagnosticabilidad: Una RdP es diagnosticable con respecto a la distribución de fallos $\Pi_{f}$ si se cumple lo siguiente:

$$
\begin{aligned}
& \left(S \in \mathcal{L}\left(Q, M_{0}\right), \wedge, S \in T_{o}\right),\left(\forall i, k \in \Pi_{f}\right)[\|S\|<\propto]: \\
& M\left(p_{f i}\right)+M\left(p_{f k}\right) \rightarrow M\left(p_{f s}\right), \wedge, J . m\left(p_{C}\right) \rightarrow \mathcal{R}(P U)
\end{aligned}
$$

Donde $S$ es una secuencia de transiciones observables y pertenece al conjunto de todas las secuencias de disparo alcanzables desde el marcado inicial $m_{0}$.

Con los datos aquí recogidos en el diagnosticador sobre los Fl's, con los cuales podemos conocer: número de veces en que ha caído en fallo, tiempo de cada fallo y tiempo total del fallo, podemos realizar unos análisis de fiabilidad, que sirvan para determinar los tiempo de uso restante del dispositivo y parámetros de vida útil de los componentes. Para este trabajo de investigación no está enmarcado como objetivo, pero es un buen inicio de trabajo futuro en el área de la fiabilidad de sistemas.

\subsection{Ejemplo Proceso de Envasado}

El proceso de envasado es el mismo que fue utilizado por [33], ver figura 5.5, este proceso está dividido en dos partes: una parte continua y una parte discreta, para este caso utilizaremos la parte discreta que es lo que nos compete para aplicar nuestra metodología; En la parte continua tiene un tanque el cual es alimentado por dos líquidos, estos son calentados por un sistema de vapor, el sistema continuo cuenta con dos válvulas alimentadoras de líquidos, una válvula de inyección de vapor, un sensor de temperatura, un sensor de nivel y un sensor de $\mathrm{Ph}$, en el cual todos juntos evalúan las condiciones ideales del líquido a envasar y su respectivos controladores. En la parte discreta del proceso de envasado se encuentran: una banda transportadora la cual es movida por un motor $M$, una válvula de llenado de líquido $\mathrm{Vll}$, sensor de llagada de botella a ser envasada $B P$, sensor de llenado de botellas $L L$ y su respectivo controlador $C$, se asume que el proceso siempre tiene botellas al inicio de la banda transportadora. El funcionamiento normal del proceso de envasado se inicia asumiendo dos cosas: que el líquido del tanque esta en óptimas condiciones y que hay botellas en la parte inicial de la banda transportadora; dadas estas condiciones el motor de la banda es encendido haciendo que las botellas 


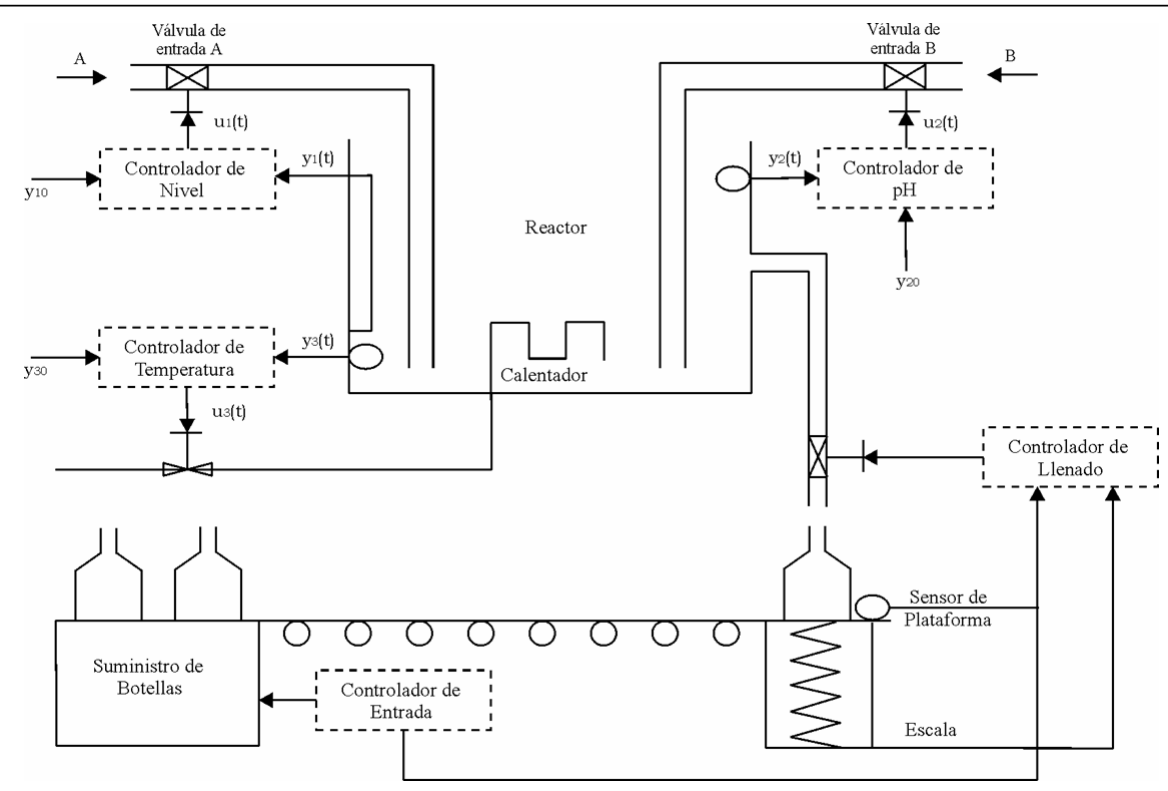

Figura 5.5: Proceso de Envasado

se desplacen hasta el lugar de envasado, el sensor $B P$ detecta la botella y por consiguiente se para el motor de la banda y se abre la válvula de llenado $\mathrm{Vll}$, cuando la botella esta llena, el sensor de llenado $L L$ informa este suceso y se cierra la válvula de llenado $\mathrm{Vll}$, estando el proceso cumplido se puede retirar la botella para que sea almacenada, éste es un proceso repetitivo que sigue actuando indefinidamente.

A continuación procedemos a aplicar la metodología, el primer paso en el ejemplo es la construcción del modelo de RdP de cada uno de los componentes del sistema, ver figura 5.6,

Como paso a seguir debemos construir el modelo general, basados en el proceso presentado en la sección 4.3. Por lo tanto tenemos:

- Paso 1. Fallos de la válvula que son: atascamiento en abierto $A A$ y atascamiento en cerrado $A C$, los fallos del motor de la banda son: fallo de motor en encendido FMon y fallo de motor en apagado FMoff; por lo tanto, obtenemos la partición de fallo siguiente: $\Sigma f_{1}=\{A A, A C\}, \Sigma f_{2}=$ $\{F M o n, F M o f f\}$

- Paso 2. Lugares de inicio, ver tabla 5.1.

- Paso 3. Asignar la marca de inicio al lugar $P_{1}$. 

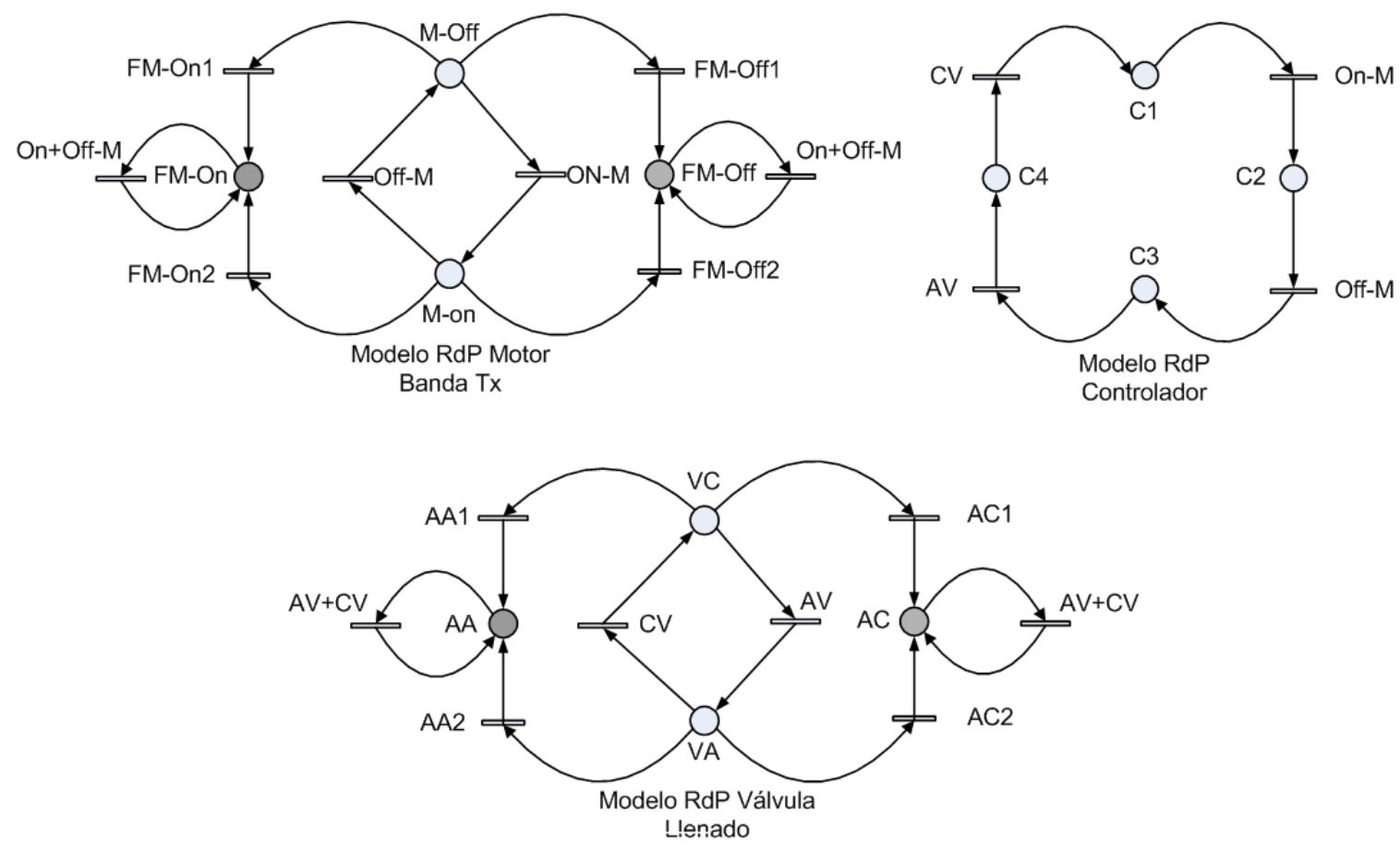

Figura 5.6: Componentes del Sistema Proceso de Envasado.

Tabla 5.1: Lugares RdPI del Sistema Completo

\begin{tabular}{|c|}
\hline$P 1=\{C 0, M O f f, V C\}$ \\
\hline$P 2=\{C 1, M O n, V C\}$ \\
\hline$P 3=\{C 2, M O f f, V C\}$ \\
\hline$P 4=\{C 3, M O f f, V A\}$ \\
\hline
\end{tabular}




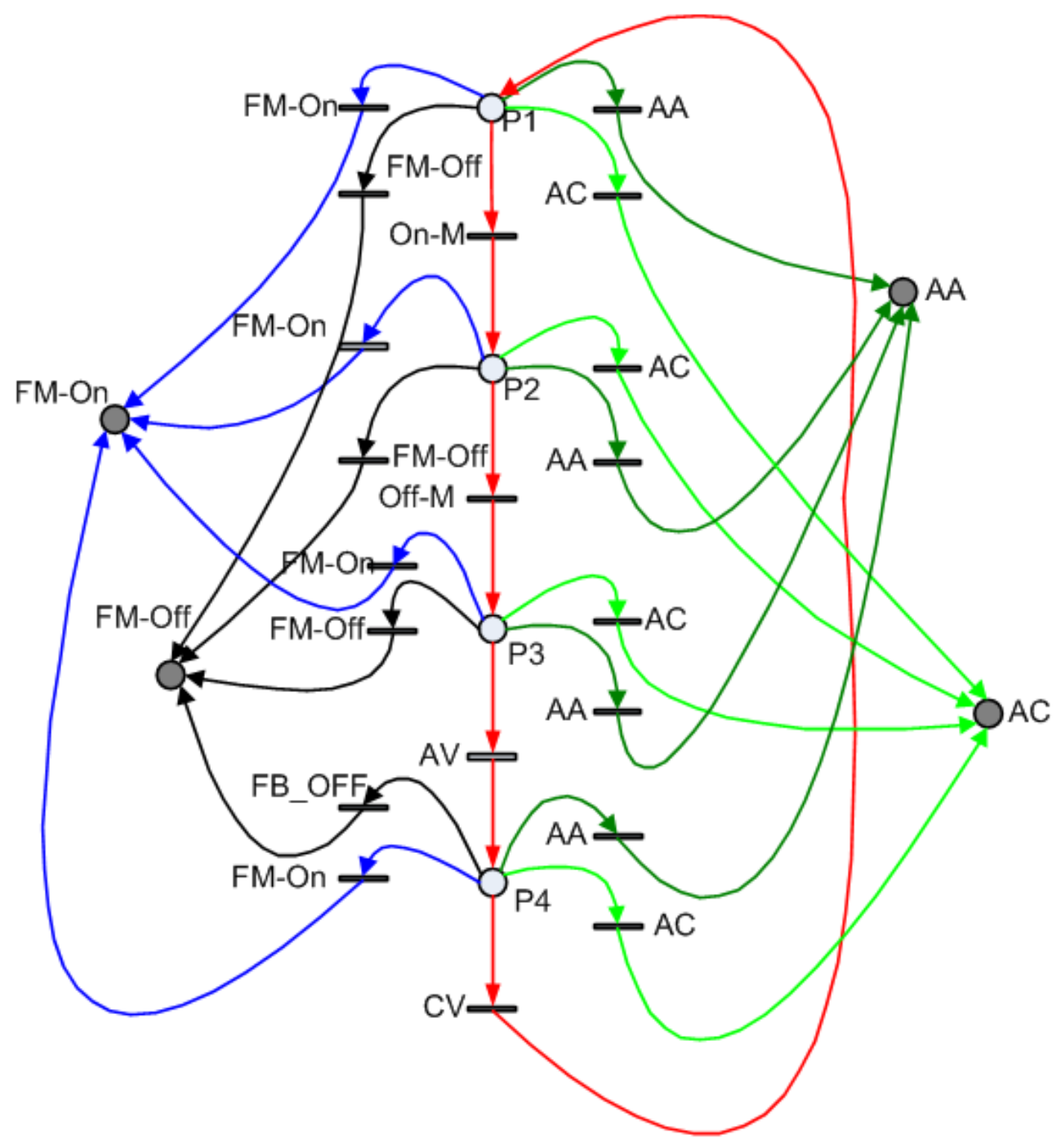

Figura 5.7: Modelo de Integración General

- Paso 4. Se construye la rama de funcionamiento normal. se va representando la evolución del funcionamiento del sistema discreto.

- Paso 5. Agregar los lugares de fallo.

- Paso 6. Adicionar las transiciones de fallo.

La RdP que representa el modelo de integración general se puede observar en la figura 5.7.

Ahora procedemos a refinar el modelo general, para ello nos basamos en lo visto en la sección 4.3 , 
Tabla 5.2: Tabla de salidas de Sensores Envasado

\begin{tabular}{|cc|}
\hline$B P$ & $L L$ \\
\hline \hline$\overline{B P}$ & $\overline{L L}$ \\
\hline$\overline{B P}$ & $L L$ \\
\hline$B P$ & $\overline{L L}$ \\
\hline$B P$ & $L L$ \\
\hline
\end{tabular}

Tabla 5.3: Salidas de la Tabla de Integración de Sensores Envasado

\begin{tabular}{|cc||c|c|c|c|}
\hline$B P$ & $L L$ & $P_{1}(\overline{B P}, \overline{L L})$ & $P_{2}(\overline{B P}, \overline{L L})$ & $P_{3}(B P, \overline{L L})$ & $P_{4}(B P, L L)$ \\
\hline \hline$\overline{B P}$ & $\overline{L L}$ & & & & \\
\hline$\overline{B P}$ & $L L$ & & & & \\
\hline$B P$ & $\overline{L L}$ & & & & \\
\hline$B P$ & $L L$ & & & & \\
\hline
\end{tabular}

- Paso 1. Sensores del sistema. Sensor de Presencia de botella BP y sensor de llenado de botella $L L$, describen el siguiente funcionamiento: presencia de botella $B P$ y no presencia de botella $\overline{B P}$, botella vacía $\overline{L L}$ y botella llena $L L$

- Paso 2. Construir el conjunto discreto de posibles salidas de los sensores que componen el sistema. $N=2$. Las combinaciones representados en la tabla 5.2, se convierten en las entradas de la tabla de integración de sensores.

- Paso 3. Definir las salidas de la tabla de integración, ver tabla 5.3, estas lecturas normales son las salidas de la tabla de integración de sensores.

- Paso 4. Construir la tabla de integración de sensores, las combinaciones se pueden ver la tabla 5.8.

Tabla 5.4: Tabla de Integración de Sensores Envasado

\begin{tabular}{|cc||c|c|c|c|}
\hline$B P$ & $L L$ & $P_{1}(\overline{B P}, \overline{L L})$ & $P_{2}(\overline{B P}, \overline{L L})$ & $P_{3}(B P, \overline{L L})$ & $P_{4}(B P, L L)$ \\
\hline \hline$\overline{B P}$ & $\overline{L L}$ & $N$ & $X$ & $X$ & $A C$ \\
\hline$\overline{B P}$ & $L L$ & $A A$ & $A A$ & $A A$ & $X$ \\
\hline$B P$ & $\overline{L L}$ & $F M_{O n}$ & $N$ & $N$ & $A C$ \\
\hline$B P$ & $L L$ & $F M_{O n}-A A$ & $A A$ & $A A$ & $N$ \\
\hline
\end{tabular}


- Paso 5. Eliminar los lugares de fallo no alcanzables basado en la tabla de integración de sensores. El refinamiento total de la RdP general se puede ver en la figura 4.4 .

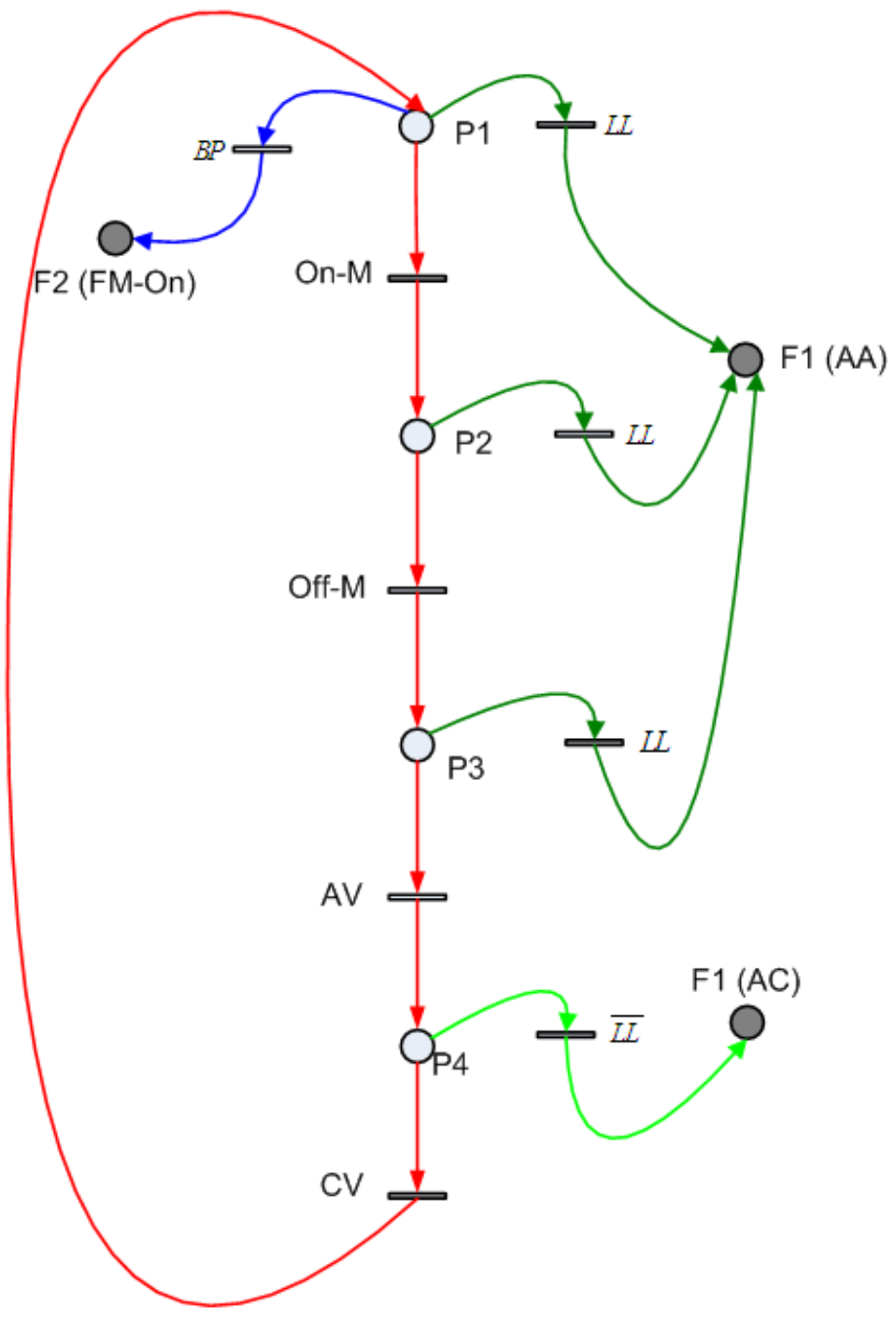

Figura 5.8: Modelo de integración Refinado

Por último se construye la $\mathrm{RdP}$ del diagnosticador, tomando como referencia el proceso planteado en 4.4.1, para el cual tenemos las etiquetas de fallo $\Delta F=$ $\left\{F_{1}, F_{2}\right\}$. En el diagnosticador se presentan dos ramas, las de fallo de válvula y motor respectivamente, también tenemos la transición de recuperación para cada lugar de fallo de la RdP, la cual permite que la RdP evolucione si las lecturas de los sensores se recuperan. Así mismo, se incorpora el lugar de conteo 
CAPÍTULO 5. ADICIÓN DE LA DINÁMICA TEMPORAL AL DIAGNOSTICADOR

temporal $P_{C}$ y la variable de tiempo $j$, esenciales en le diagnostico de Fallos Intermitentes. el diagnosticador se puede observar en la figura 5.9. 


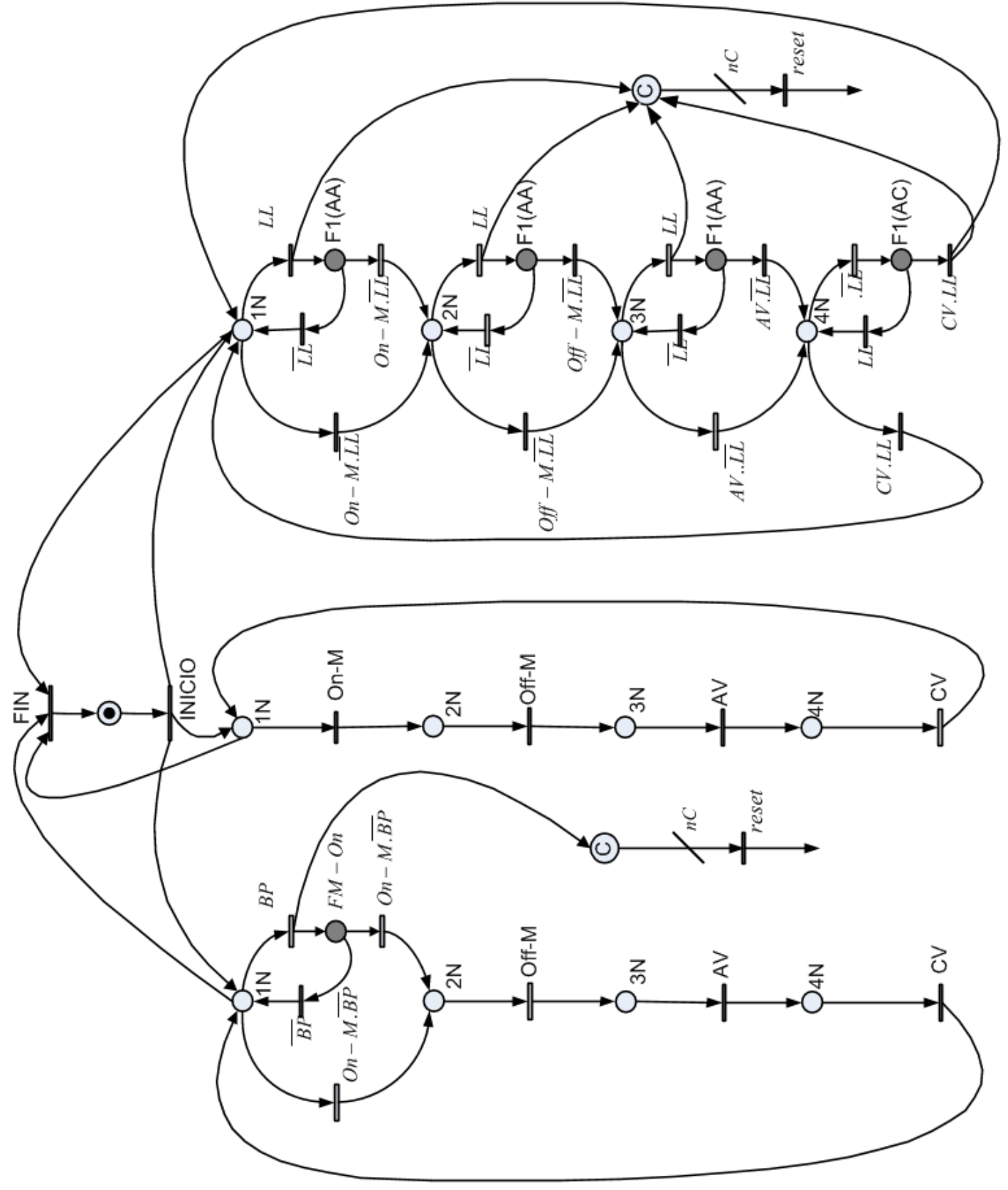

Figura 5.9: Diagnosticador 


\section{Capítulo 6}

\section{Proceso de Construcción del Diagnosticador usado en Sistemas Híbridos}

\subsection{Introducción}

Un sistema híbrido es un sistema en el cual el comportamiento de interés es determinado por la interacción de procesos de diferentes características, en particular, interactuando dinámica continua y discreta. Sistemas híbridos típicamente generan señales mezcladas que consiste de combinaciones de señales de valor discreto y continuo. Ademas, estas señales de valor discreto o continuo dependen de variables independientes tales como tiempo, el cual también puede ser de valor continuo o discreto. Otra distinción que puede ser hecha, es que algunas de las señales pueden ser manejadas por tiempo, mientras que otras puede ser manejadas por eventos, en una manera asincrónica.

El comportamiento dinámico de tales sistemas híbridos es capturado en modelos híbridos. En un proceso de fabricación, por ejemplo, las partes pueden ser procesadas en una máquina en particular, pero solo el arribo de una pieza activa el proceso; es decir, el proceso de fabricación se compone de la dinámica manejada por eventos de las partes moviéndose entre las diferentes máquinas y la dinámica manejada por los tiempos de los procesos entre máquinas particulares. En sistemas híbridos en el pasado, la dinámica de manejo de eventos fue estudiada por separado de la dinámica manejada por tiempo, la primera usando modelos de Autómata o Redes de Petri (también PLC, expresiones lógi- 
cas, etc) y la segunda vía ecuaciones diferencia o diferenciales. Para entender completamente el comportamiento del sistema y encontrar especificaciones de alto rendimiento, se necesita modelar todas las dinámicas juntas con sus interacciones. Problemas tales como la optimización del proceso de fabricación completo, podrán ser abordados de una manera significativa. Hay, por supuesto, casos donde la dinámica de manejo de eventos y manejo de tiempos, no están bien contempladas o la demanda en el rendimiento del sistema es difícil de encontrar, en esos casos se considera simplemente separar los modelos para los distintos fenómenos. Sin embargo, los modelos híbridos deben ser usados cuando hay significante interacción entre la parte discreta y continua, y altas especificaciones de rendimiento van a ser encontradas por el sistema.

La realidad de los procesos hoy día es su alta complejidad, en ellos se puede encontrar diversidad de elementos que lo componen: controladores, transmisores, sensores, actuadores, entre otros. La razón mas importante para el uso de modelos híbridos es reducir la complejidad. Esto se logra mediante la incorporación de modelos de procesos dinámicos en los diferentes niveles de abstracción. Otra razón es evitar enfrentarse directamente a un conjunto de ecuaciones no lineales, se puede optar por trabajar con conjuntos de ecuaciones más sencillas (lineales) y cambiar entre estos modelos más simples. Éste es un enfoque bastante común en la modelización de fenómenos físicos. Los sistemas híbridos han sido motivados en parte por el desarrollo de los resultados de la investigación sobre el control de SED's y en el control adaptativo en los años 1970 y 1980 y por el renovado interés en la formulación de control óptimo en sistemas de datos muestreados y de control digital. Los sistemas híbridos representan un área muy difícil de la investigación que abarca una variedad de problemas desafiantes que pueden abordarse en varios niveles de detalle y sofisticación. Diferentes tipos de modelos se utilizan, desde modelos detallados que pueden incluir ecuaciones y tablas de búsqueda, que son excelentes para la simulación, pero no fácilmente susceptibles de análisis, los modelos que son también buenos para el análisis pero no fácilmente susceptibles a la síntesis, modelos de control, los modelos de verificación, entre otros. 


\section{Enfoques para el Análisis y Diseño de Sistemas Híbridos.}

Los enfoques actuales a los sistemas híbridos difieren en cuanto al énfasis en la complejidad de la dinámica continua y discreta, y en si hacen hincapié en el análisis y la síntesis de resultados, o el análisis solamente, o solo simulación. En un extremo del espectro hay planteamientos de los sistemas híbridos que representa extensiones del sistema de ideas teóricas de los sistemas (con variables de valor continuo y con valores de tiempo continuo) que se describen por ecuaciones diferenciales ordinarias, a fin de incluir en tiempo discreto las variables que presentan saltos o amplían los resultados a los sistemas conmutados. Típicamente, estos enfoques son capaces de hacer frente a la dinámica continua compleja. Su énfasis principal ha sido sobre la estabilidad de los sistemas con discontinuidades. En el otro extremo del espectro hay planteamientos de los sistemas híbridos incorporados en los modelos de software y los métodos que representa la extensión de las metodologías de verificación de sistemas discretos de sistemas híbridos (Como la planteada en esta investigación). Típicamente, estos enfoques son capaces de hacer frente a la dinámica discreta descrito por autómatas finitos y hacer hincapié en los análisis de resultados (verificación) y metodologías de simulación. Hay metodologías adicionales que abarcan el resto del espectro desde que combinan los conceptos de sistemas de control continuo que se describen por ecuaciones diferenciales lineales y no lineales, y desde el control supervisorio de SED's que se describen por autómatas finitos y redes de petri para obtener, con éxito variable, análisis y síntesis de los resultados.

Es muy importante contar con buenas herramientas de software para la simulación, análisis y diseño de sistemas híbridos, que por su naturaleza son sistemas complejos. Esta necesidad ha sido reconocida por la comunidad de los sistemas híbridos y varias herramientas de software han sido desarrolladas. La lista de herramientas de software, cambia de forma dinámica para adaptarse a los progresos en la investigación de sistemas híbridos. Entre otras tenemos: Matlab/Simulink/Stateflow proporcionan herramientas para el modelado visual y simulación de sistemas híbridos que pueden incluir tiempo continuo, tiempo discreto, y la dinámica manejada por eventos. Ptolemy II [23] es un conjunto de paquetes de software soportando el diseño y modelado concurrentes heterogéneo. Es compatible con máquinas de estados finitos, sistemas de eventos 
discretos, y sistemas de tiempo continuo, permite el modelado y simulación de sistemas híbridos. El lenguaje orientado a objetos Modélica [1], ha sido desarrollado para modelar sistemas físicos y proporciona paradigmas de modelado para sistemas híbridos. HCC es un lenguaje orientado a objetos, inicialmente fue desarrollado en Xerox PARC, y después extendido a la NASA Ames, donde ha sido utilizado en diversas aplicaciones espaciales. El lenguaje de programación Shift [9] se ha desarrollado para describir las redes dinámicas de los autómatas híbridos. Su principal motivación fue la especificación y análisis de aplicaciones de automoción. Charon es un lenguaje para modelado jerárquico y modular de los sistemas híbridos [2]. Hytech es una herramienta de verificación de sistemas híbridos basado en la teoría de autómatas híbridos lineal [42]. Kronos [21] y UPPAAL [54] son herramientas de verificación para sistemas de tiempo real modelado por autómatas temporizados. Además, muchos grupos de investigación están desarrollando herramientas de software para apoyar su propio trabajo.

\subsection{Herramienta de Diagnóstico}

La complejidad de un sistema híbrido es la razón fundamental para que se tome la decisión de migrar de una herramienta de diagnóstico de fallos, diseñado inicialmente solo para SED's a sistemas híbridos. Se busca aplicar los conceptos utilizados en SED's en sistemas continuos, dando como resultado la integración del sistema general de diagnóstico en un sólo diagnosticador, con la función de evaluar su funcionamiento de una manera on-line. Los conceptos de diagnóstico de fallos, presentados en el capítulo 4, son la base fundamental para desarrollar la técnica en sistemas híbridos, iniciamos esta metodología haciendo un repaso de los elementos fundamentales, previos a construir el diagnosticador. El modelo que representa el sistema debe contener el funcionamiento normal y de fallo del sistema.

Sea $R=\left(P, T\right.$, Pre, Post, $\left.m_{0}\right)$, la RdP que representa el modelo del sistema a diagnosticar, donde el comportamiento normal y de fallo son considerados. Además, las transiciones $T$ pueden ser clasificadas como no observable $T_{u o}$ y observable $T_{o}$, siendo $T=T_{o} \cup T_{u o}$. Transiciones observables son las transiciones que son activadas por los eventos de control $T_{C}$ (comandos del supervisor) o por la instrumentación empleada en el proceso $T_{S}$. Por lo tanto, $T_{o}=T_{C} \cup T_{S}$. 
Por otro lado, las transiciones no observables son esas que no son detectadas por el sistema cuando ocurren. Así, las transiciones de fallo $T_{f}$ son un subconjunto de las transiciones no observables $T_{f} \subseteq T_{u o}$. $T_{f}$ pueden ser clasificadas dentro de un conjunto disjunto, correspondiente a diferentes tipos de fallo que pueden ocurrir en el sistema, consecuentemente $T_{f}=T_{f_{1}} \cup \ldots \cup T_{f_{N}} . N$ denota el número de componentes del sistema. Acorde a esto, cuando un fallo de tipo $F_{i}$, una transición del conjunto $T_{f_{i}}$ será activada. La distribución de fallos $\Pi_{f}$ define el conjunto de fallos a ser evaluado en el sistema. Hay que recordar que la representación con redes de petri híbridas, es diferente si se representa un lugar o transición de la dinámica continua a representar un lugar o transición discreto, así mismo, el marcado y disparo de una lugar continuo varía, ver figura 3.3.

\subsubsection{Proceso de Construcción del Modelo y Diagnosticador}

La construcción del modelo y diagnosticador mantiene las mismas pautas presentadas en el capítulo 4, ahora se integra la parte continua al sistema para extender el diagnóstico a sistemas híbridos. Esta sección muestra el proceso para construir el modelo y diagnosticador. El sistema está compuesto de varios subsistemas trabajando juntos y compartiendo diferentes funcionalidades. Se deben seguir los siguientes pasos:

\section{Paso 1. Dividir el Sistema en Subsistemas.}

El sistema $H$ puede ser dividido en $M$ subsistemas con cercana relación entre ellos, dependiendo de su comportamiento. Esto permite reducir la complejidad de los sistemas híbridos. Así

$$
H=H_{1} \cup H_{2} \cup \ldots \cup H_{M}
$$

$j=1, \ldots M, M$ es el número de subsistemas que componen el sistema.

\section{Paso 2. Construir el modelo de RP de los componentes de cada subsiste-} ma.

En cada uno de los subsistemas identificados $H_{j}, j=1, \ldots, M$ dependiendo si es un subsistema discreto o continuo, la forma de representar el modelo varía 
y se debe utilizar los procedimientos planteados en el capítulo 3. En los modelos se debe tener en cuenta el comportamiento normal y de fallo. Al mismo tiempo, se tiene que definir los fallos a evaluar en cada componente del subsistema.

Sea $R_{i}^{j}=\left(P_{i}, T_{i}\right.$, Pre, Post, $\left.m_{0}\right)$, la RdP marcada del $i$-componente correspondiente al subsistema $j, i=1, \ldots, N, N$ es el número de componentes en el subsistema $j . P_{i}$ es el conjunto de lugares, $T_{i}$ es el conjunto de transiciones, Pre/Post son las aplicaciones de la incidencia de entrada/salida y $m_{0}$ es el marcado inicial. Es importante notar que se debe mantener la sincronía de funcionamiento del proceso completo. Para diferenciar los lugares y transiciones de fallo, éstas se deben representar con círculos y barras sombreados respectivamente.

\section{Paso 3. Operación de integración.}

La siguiente expresión sera usada para denotar la operación de integración para el subsistema $\widetilde{Q^{j}}=\left(\widetilde{P^{j}}, \widetilde{T^{j}}, \widetilde{\text { Pre }^{j}}, \widetilde{\text { Post }^{j}}, m_{0}^{j}\right)$. Por lo tanto, $\widetilde{Q^{j}} \tilde{r}$ epresenta el comportamiento del subsistema $H_{j}$ a través de un único modelo de RdP, el cual incluye $N_{j}$ diferentes modelos de RdP correspondientes a sus componentes. Este modelo integra el comportamiento normal y de fallo del sistema, las transiciones (observable $T_{o}$ y no observable $T_{u o}$ ) pueden ocurrir en cualquier lugar del modelo. Sea $\widetilde{P^{j}}$ y $\widetilde{T^{j}}$ los conjuntos de lugares y transiciones de $\widetilde{Q^{j}}$ del subsistema $j$, y son compuestos por la unión de lugares y transiciones del componente respectivo, como sigue:

$$
\widetilde{P^{j}}=\bigcup_{i=1}^{N} P_{i} \quad \text { y } \quad \widetilde{T^{j}}=\bigcup_{i}^{N} T_{i}
$$

El siguiente proceso tiene que ser completado para construir el modelo de integración. Así, para cada uno de los subsistemas $M$ es requerido:

- Paso 3.1. Definir el conjunto de fallos $F$ para cada componente del subsistema. $F=\left\{F_{1}, F_{2}, \ldots, F_{N}\right\}$.

- Paso 3.2. Definir el lugar inicial $p_{0}^{j}$. Éste brinda los lugares iniciales de cada componente del subsistema. $P_{0}^{j}=\left\{p_{0}^{1}, p_{0}^{2}, \ldots, p_{0}^{N}\right\}$.

- Paso 3.3. 3. Asignar la marca al lugar inicial $p_{0}^{j}$. Éste define el punto de inicio para la evolución del subsistema representado. 
- Paso 3.4. Construir la rama correspondiente al comportamiento normal $R_{N}^{j}$. Esta designa la interacción y evolución de los componentes. Las transiciones son solamente compuestas por comandos del controlador $T_{C}$. El conjunto de lugares del subsistema es compuesto de los lugares de cada componente.

- Paso 3.5. Adicionar los lugares de fallo $P_{f}^{j}$. Un lugar por cada fallo desde el conjunto $F$ tiene que ser generado.

- Paso 3.6. Adicionar las transiciones de fallo $T_{f}^{j}$. En cada lugar de la RdP general del subsistema $j$ se puede presentar la evolución de los fallos, es decir, desde cada lugar $p_{i}^{j}$ de la RdP general, una transición de fallo $t_{f}^{j}$ es por lo tanto adicionada: $\forall p_{i}^{j}: p_{i} \in P^{j} \rightarrow \exists\left(p_{i}, t_{j}\right) \in F, F$ es el conjunto de fallos del sistema.

- Paso 3.7. Conectar los lugares normales $p_{i}^{j}$ a las transiciones de fallo $p_{f}^{j}$, y las transiciones de fallo $t_{f}^{j}$ a los lugares de fallo $p_{f}^{j}$ usando arcos.

Los lugares de fallo agregados al modelo, se constituyen en lugares de bloqueo de la RdP, conocidos como lugares sumidero. Dado que la construcción de este proceso de FD es sistemático, esto no representa aún un problema, ya que, quien realmente va a cumplir la función de supervisor es el diagnosticador y para esta parte, la RdP habrá sufrido las transformaciones necesarias, donde se corrigen estos bloqueos y se garantiza poder tener una RdP viva, es decir, libre de bloqueos.

\section{Paso 4. Refinar el Modelo General.}

Es necesario considerar solo la parte observable del modelo general $\widetilde{Q^{j}}$, Por lo tanto, $\widetilde{Q^{j}}=\left(\widetilde{P^{j}}, \widetilde{T^{j}}, \widetilde{\text { Pre }^{j}}, \widetilde{\text { Post }^{j}}, \widetilde{m_{0}^{j}}\right)$ debe ser transformada al modelo refinado $Q^{j}=\left(P^{j}, T^{j}, \operatorname{Pre}^{j}\right.$, post $\left.^{j}, m_{0}^{j}\right)$. Este modelo está solo compuesto de lugares y transiciones observables. Acorde a esto, las transiciones de fallo tienen que ser reemplazadas por lecturas de los sensores $T_{S}$. El siguiente proceso debe ser seguido para refinar el modelo:

- Paso 4.1. Identificar los sensores del sistema. Cada subsistema tiene $N s_{j}$ sensores. La idea fundamental de la herramienta es aprovechar la información disponible del subsistema leída por los sensores, con ésta infor- 
Tabla 6.1: Tabla de salidas de Sensores

\begin{tabular}{|cc|}
\hline$S_{1}$ & $S_{2}$ \\
\hline \hline$\overline{L_{1}}$ & $\overline{L_{2}}$ \\
\hline$\overline{L_{1}}$ & $L_{2}$ \\
\hline$L_{1}$ & $\overline{L_{2}}$ \\
\hline$L_{1}$ & $L_{2}$ \\
\hline
\end{tabular}

mación se identifica la aparición de posibles condiciones anormales del sistema.

- Paso 4.2. Construir el conjunto discreto de salidas de sensor. Se debe suponer que el sistema de sensores debe entregar solo variables discretas. Dado el conjunto $N s$ sensores del subsistema de interés, identificar sus salidas y con ellas construir la combinación posible de las lecturas sensoriales. $Y$ representa el número de combinaciones de las lecturas de sensores. Desde ahí, $Y$ son las entradas para la tabla de integración de sensores. $|Y|=2^{N s}$. Para una mejor ilustración de la construcción de las posibles salidas de sensores, suponemos que el sistema posee dos sensores, el estado discreto de cada sensor es $L \circ \bar{L}\left(\begin{array}{l}1 \\ 1\end{array}\right.$ 0 $)$ según corresponda. Para el ejemplo tendremos $2^{2}=4$ combinaciones de salidas de sensores, representados en la tabla 6.1. Las combinación $Y$ dada anteriormente se convierte en las entradas de la tabla de integración de sensores.

- Paso 4.3. Definir las salidas de la tabla de integración. Una salida en la tabla de integración de sensores es adicionada para cada lugar de la rama de comportamiento normal en la RdP de cada subsistema. Cada lugar representa el estado actual de las lecturas de los sensores, por ejemplo, el lugar $P_{3}^{j}$ representa a las lecturas de los componentes $X, \bar{Y}$, entonces, $P_{3}^{j}=(X, \bar{Y})$, muestra que en el lugar normal $P_{3}, X$ está en este lugar en un estado positivo y $Y$, mantienen un estado negativo. Ver la tabla 6.2

- Paso 4.4. Construir la tabla de integración de sensores $h_{j}=\widetilde{P} \rightarrow \widetilde{Y}_{j}$, $j=1, \ldots, M$, donde $\widetilde{Y}_{j}$ representa el conjunto discreto de salidas de sensor 
CAPÍTULO 6. PROCESO DE CONSTRUCCIÓN DEL DIAGNOSTICADOR USADO EN SISTEMAS HÍBRIDOS

Tabla 6.2: Salidas de la Tabla de Integración de Sensores Subsistema

\begin{tabular}{|cc||c|c|c||c|}
\hline$S_{1}$ & $S_{2}$ & $P_{1}^{j}(\bar{X}, Y)$ & $P_{2}^{j}(X, Y)$ & $P_{3}^{j}(X, \bar{Y})$ & $\ldots . P_{i}^{j}(X, Y)$ \\
\hline \hline$\overline{L_{1}}$ & $\overline{L_{2}}$ & & & & \\
\hline$\overline{L_{1}}$ & $L_{2}$ & & & & \\
\hline$L_{1}$ & $\overline{L_{2}}$ & & & & \\
\hline$L_{1}$ & $L_{2}$ & & & & \\
\hline
\end{tabular}

Tabla 6.3: Tabla de Integración de Sensores Subsistema

\begin{tabular}{|cc||c|c|c||c|}
\hline$S_{1}$ & $S_{2}$ & $P_{1}^{j}(\bar{X}, Y)$ & $P_{2}^{j}(X, Y)$ & $P_{3}^{j}(X, \bar{Y})$ & $\ldots . P_{i}^{j}(X, Y)$ \\
\hline \hline$\overline{L_{1}}$ & $\overline{L_{2}}$ & $F$ & $F$ & $X$ & $F$ \\
\hline$\overline{L_{1}}$ & $L_{2}$ & $N$ & $X$ & $F$ & $X$ \\
\hline$L_{1}$ & $\overline{L_{2}}$ & $X$ & $X$ & $N$ & $N$ \\
\hline$L_{1}$ & $L_{2}$ & $F$ & $N$ & $F$ & $N$ \\
\hline
\end{tabular}

posible del subsistema $j$, esto define:

$$
Y=\prod_{i=1}^{N} Y_{j}
$$

$h_{j}$ es definida por $h(P)=\left(h_{1}\left(P_{j}\right), h_{2}\left(P_{j}\right), \ldots, h_{N}\left(P_{j}\right)\right)$ y corresponde a a tabla de integración de sensores. Las entradas de la tabla de integración $Y$ y las lecturas esperadas de los sensores de cada lugar de la rama normal deben ser comparadas, es decir, si las lecturas del sensor y las lecturas esperadas son las misma, entonces, $h_{1}\left(P_{j}\right)$ es clasificada como normal $N$. Por otra parte, Si las lecturas son diferentes, estas pueden indicar a un fallo $F$ o un valor indeterminado $X$, debido a que ellas no tienen información para usar, $Y_{j}=Y_{j} N \bigcup Y_{j} F \cup Y_{j} X$. Por lo tanto, en cada lugar del modelo se pueden definir las salidas de sensores como $N$ (normal), $F$ (fallo) y $X$ (no determinado). Ver tabla 6.3.

- Paso 4.5. Reemplazar las transiciones de fallos y remover los lugares no alcanzables. Las transiciones de fallo del modelo de RdP general $\widetilde{Q^{j}}$ tienen que ser reemplazadas por las lecturas de los sensores. Los lugares de fallo que no pueden ser identificados porque la información disponible no 
permite habilitar el marcado del lugar, deben ser removidos de $\widetilde{Q^{j}}$.

$$
\forall p_{i}: p_{i} \in P \rightarrow M\left(p_{i}\right) \notin R\left(Q, m_{0}\right)
$$

Finalmente, el modelo de integración refinado de cada subsistema esta compuesto de lugares normales $P_{i}^{j}=p_{i}^{1} \cup p_{i}^{2} \cup \ldots \cup p_{i}^{N}$ y lugares de fallo $p_{f}$ dados en $\Pi_{f}, P^{j}=P_{N}^{j} \cup P_{F}^{j}$. Las transiciones $T^{j}$ incluyen eventos de control $T_{C}$ y las lecturas de los sensores $T_{S}$ consideradas en la tabla de integración; $T=T_{C} \cup T_{S}$. En conclusión, el modelo refinado de cada subsistema estará compuesto solo por lugares observables.

\section{Paso 5. Construcción del diagnosticador.}

El diagnosticador es una RdP implementada considerando el modelo refinado del sistema. Sea $Q^{j}=\left(P^{j}, T^{j}, \operatorname{Pre}^{j}\right.$, post $\left.^{j}, m_{0}^{j}\right)$ la $\mathrm{RdP}$ que representa el modelo refinado de cada uno de los subsistemas $H_{j}$ del sistema completo $(j=1, \ldots, M)$ y $t_{f}$ la transición final de la secuencia $S$, definida como sigue:

$$
\mathcal{L}\left(T_{f_{i}}\right)=\left\{S t_{f} \in L: t_{f} \in T\right\}
$$

$\mathcal{L}\left(T_{f_{i}}\right)$ denota el conjunto de todas las secuencias de $L$ (lenguaje que representa el comportamiento del sistema) que finaliza en una transición de fallo, correspondiente a la clase $t_{f_{i}}$. Considerando que $t_{f} \in T$ y $S \in T^{*}$. La notación $t \in S$ es usada para denotar que $t$ es una transición de la secuencia $S$, también escribiendo que $t_{f} \in T$ para cualquier $t_{f}$.

En el contexto de la metodología desarrollada en esta investigación se deben realizar los siguientes supuestos:

- Existe una transición definida en cada lugar $p \in P$, por lo tanto, la Red de Petri no alcanzará ningún lugar sumidero, evitando que la red quede en un estado de bloqueo.

- No existe en $Q^{j}$ transiciones no observables $T_{u o}$. Esta condición esta garantizada, gracias al desarrollo de un modelo general refinado, es decir, que solo contiene transiciones observables. 
CAPÍTULO 6. PROCESO DE CONSTRUCCIÓN DEL DIAGNOSTICADOR USADO EN SISTEMAS HÍBRIDOS

Adicionalmente a esto, una etiqueta tiene que ser asignada a cada fallo del sistema. Produciendo un conjunto de etiquetas de fallo definidas acorde a la expresión: $\Delta F=F_{1}, F_{2}, \ldots, F_{m}$. En general, un conjunto de etiquetas $\Delta$ esta compuesto de etiquetas normales $N$ y etiquetas de fallo $F$, así $\Delta=N \cup F$. En general, el diagnosticador para el sistema $H$ es una RdP de la forma $Q_{d}=$ $\left(P_{d}, T_{d}\right.$, Pre, Post $\left., m_{0}, p_{0}, t_{0}, t_{\text {end }}\right)$, tal que:

- $P_{d}=\left\{P_{1}, P_{2}, \ldots, P_{n}\right\}$ es el conjunto de lugares finito y no vacío;

- $T_{d}=\left\{T_{1}, T_{2}, \ldots, T_{m}\right\}$ es el conjunto de transiciones finito y no vacío;

- $P \cap T=\emptyset$, es decir que los conjuntos $P_{d}$ y $T_{d}$ son disjuntos;

- Pre $: P \times T \rightarrow\{0,1\}$ es la aplicación de la incidencia de entrada;

- Post : $T \times P \rightarrow\{0,1\}$ es la aplicación de la incidencia de salida;

- $m_{0}$ es el marcado inicial;

- $p_{0}$ es el lugar de inicio donde reside el marcado inicial;

- $t_{0}$ es la transición de inicio del diagnosticador;

- $t_{\text {end }}$ es la transición que finaliza el funcionamiento del diagnosticador.

El conjunto de lugares $P_{d}$ del diagnosticador es una extensión del conjunto de lugares del modelo general. Así, un lugar $p$ de $Q_{d}$ es de la forma $p_{d}=\left(p_{i}, l_{i}\right)$ donde los lugares corresponden a lugares observables, $p_{i} \in P_{o}$ y la etiqueta $l_{i}$ corresponde al conjunto de etiquetas $\triangle$. Las etiquetas para un lugar específico serán de la forma $l_{i}=\{N\} \vee\left\{F_{i}\right\}$. Por lo tanto, un lugar $p_{d}$ puede tomar una etiqueta de funcionamiento normal o de fallo.

Un observador de cada modelo refinado $Q_{j}$, ofrece la estimación de lugar actual del sistema híbrido $H$, después de la aparición de cada transición observable, el diagnosticador $Q_{d}$, siguiendo la dinámica de las $\mathrm{RdP}$, puede entenderse conceptualmente como un observador extendido, donde se añade a cada estimación de lugar, una etiqueta del tipo de las mencionadas anteriormente, las etiquetas adjuntas indican el estado del componente, si está en modo de fallo o modo normal, los fallos se diagnostican con la validación de las etiquetas, las cuales están unidas a su respectivo lugar. 
Las funciones fundamentales para construir el diagnosticador son:

Función de Asignación de Etiqueta $L A: P_{o} \times \triangle \times T^{*} \rightarrow \triangle$. Dados $p \in P_{0}$, $l \in \triangle \mathrm{y} S \in L(Q, P), L A$ asigna la etiqueta $l$ sobre $S$ (secuencia de transiciones) iniciando desde $p$ y siguiendo la dinámica de $Q^{j}$, acorde a:

$$
L A(P, l, S)=\left\{\begin{array}{l}
\{N\} \text { si } \forall i\left[T_{f_{i}} \notin S\right] \\
\{F\} \text { si } \forall i\left[T_{f_{i}} \in S\right]
\end{array}\right.
$$

Esto significa que la red de petri diagnosticadora (RdPD) $Q_{d}$ asigna a cada lugar su respectiva etiqueta, dependiendo de la transición disparada, sera $N$ (Normal) si una $T_{0}$ es disparada en la secuencia $S$ o será $F_{i}$ si ha sido disparada una transición de fallo $T_{f}$.

Cabe señalar que los lugares sumidero aparecen en el modelo de integración $Q^{j}$. Si el marcado cae dentro de un lugar sumidero, la RdP será bloqueada. En orden a evitar este problema, una función de Expansión de Fallo $(F E)$ tiene que ser creada, aprovechando las ventajas de la concurrencia de las RdP.

Consecuentemente, La función de Expansión de Fallo es definida acorde a la siguiente expresión: $E F: R_{N} \times F_{i} \rightarrow R_{F}$. Donde, $R_{N}$ es la rama de funcionamiento normal y $R_{F}$ es la rama de funcionamiento de fallo. Para cada conjunto de fallo $F_{i}$, una nueva rama de fallos en la RdP sera creada para cumplir con la norma de supervisar los fallos individualmente. El diagnosticador $Q_{d}$ tendrá tantas ramas como número de fallos tiene el sistema. $R_{Q d}$ especifica el número total de ramas del diagnosticador.

$$
R_{Q d}=\sum_{i=1}^{M} R_{f_{i}}
$$

\section{El procedimiento para construir el diagnosticador es el siguiente:}

- Paso 5.1. Definir el lugar de inicio $p_{0}$. El lugar de inicio $p_{0}$ estará compuesto por el respectivo lugar $p$ y por la etiqueta normal $N,(p,\{N\})$, y por consiguiente alberga la marca del marcado inicial.

- Paso 5.2. Adicionar la transición de inicio $t_{0}$. Conectar $p_{0}$ a $t_{0}$ por medio de un arco. Cumple con la función de permitirle al operador o supervisor del sistema, el inicio de la RdPD. 
- Paso 5.3. Agregar la transición de final $T_{\text {end }}$. Conectar $t_{\text {end }}$ a $p_{0}$ por medio de un arco. Cumple la función de finalizar el funcionamiento del diagnosticador.

- Paso 5.4. Adicionar la rama da fallo $R_{F}$. Para cada fallo de $\Pi_{f i}$, una rama de fallo es construida acorde a la función de expansión de fallo $E F$. Cada rama incluye una rama de funcionamiento normal en paralelo a la rama de fallo basado en que desde cada lugar puede disparar una transición de fallo $t_{f}$ o una transición normal $t_{N}$. Cuando una $t_{f}$ es disparada, la RdPD va a un lugar de fallo $p_{f}$ y consecuentemente la función $L A$ asigna una etiqueta. En orden a continuar con la evolución de la RdP, las lecturas de los sensores mas las transiciones de control deben ser disparadas, $\left(T_{S}+T_{C}\right)$. Estas ramas de fallo, funcionará de manera paralela a la normal, si aparece una lectura anormal en los sensores del sistema, es decir un fallo, la rama se quedará en ese lugar de fallo y el supervisor evidenciará el mismo, tomando las correcciones necesarias. Todas las ramas funcionan de manera independiente, lo que nos permite diagnosticar múltiples fallos en el mismo diagnosticador.

- Paso 5.5. Adicionar las Transiciones de Recuperación $t_{R}$. Para cada uno de los lugares de fallo, su evolución puede ir en un camino de funcionamiento normal (paso anterior) o las lecturas de los sensores pueden cambiar desde el estado de fallo a estado normal. Por lo tanto, una transición de recuperación $t_{R}$ debe ser adicionada a la rama y la marca del lugar debe retornar al lugar anterior.

$$
\left(\forall p_{f_{i}} \in P_{F}\right)\left(\exists t_{R_{i}} \in T_{R}: t_{R}=t_{S}\right)\left(p_{f_{i}} \times t_{R_{i}} \rightarrow p_{N(i-1)}\right)
$$

- Paso 5.6. Conectar $t_{0}$ y $t_{\text {end }}$ a las ramas. Un arco debe ser conectado desde $t_{0}$ a cada lugar inicial de las ramas $\mathrm{y}$, en la misma forma, desde cada lugar inicial de la rama a $t_{\text {end }}$. En este paso, el peso de los arcos son usados para evitar conflictos en la RdP; por lo tanto, el peso de los arcos debe ser igual al número de fallos mas uno (correspondiente a la rama normal de su respectivo subsistema). 
- Paso 5.7. Dinámica Temporal para diagnóstico de fallos intermitentes. Para cada lugar de fallo, una variable de tiempo $t$ es adicionada. Ésta es iniciada cuando la marca llega al lugar de fallo y finaliza cuando la marca se va, porque la transición de recuperación es disparada. Este tiempo almacenado por $t$ debe ser adicionado a otro cuando la rama cae en fallo. $\forall p_{f_{i}} \in P_{F}, \exists t \rightarrow p_{f_{i}}=\left(M\left(p_{i}\right), t_{i}\right)$, donde $i=1, \ldots, Z, Z$ es el número de fallos generados por el sistema. Para cada rama de fallo del diagnosticador, un lugar de conteo temporal $p_{C}$ es adicionado. Cuando una transición de fallo es disparada, una marca debe ser adicionada a $p_{C}$. Esto significa que el número de fallos están almacenados dentro del lugar $p_{C}$. Y la suma de los tiempos registrados por la variable $t$ son almacenados en $p_{C}$. Entonces, desde cada transición de fallo de la rama de fallo, un arco debe ser conectado al lugar de conteo temporal $p_{C}$. El número de marcas en el lugar de conteo temporal es la suma de las marcas registradas en cada lugar de fallo. También en este lugar de conteo temporal $p_{C}$, se realizará la sumatoria de los tiempos que suceden en cada unos los lugares de fallo, almacenados en la variable $t$. Lo anterior indica que desde cada lugar de fallo de la rama, debe salir un arco que finaliza en el lugar de conteo temporal de la rama $p_{C}$.

$$
\begin{gathered}
\forall R_{F} \in R, \quad \exists p_{C}: t_{f_{i}} \rightarrow p_{C}, \wedge, t=\sum_{i=1}^{Z} t_{i} \\
m\left(p_{C}\right)=\sum_{i=1}^{Z} m\left(p_{f_{i}}\right), \quad i=1, \ldots, Z
\end{gathered}
$$

- Paso 5.8. Reseteo de la dinámica temporal. Cuando el operador, (es decir, el piloto) para el diagnosticador para reemplazar o reparar un componente, la variables $t$ y el lugar de conteo temporal $p_{C}$ deben ser reseteados. Un arco debe ser adicionado desde $p_{C}$ a $t_{\text {end }}$ para este propósito. Otro arco iniciando desde $t_{\text {end }}$ pero sin lugar de llegada debe ser adicionado también. El peso de este arco es igual al número de marcas almacenadas en $p_{C}$. De está manera, $p_{C}$ está vacío (no tiene marcas) y la variable $t$ es convertida a cero. Por consiguiente, se podría iniciar una nueva etapa de diagnóstico. En conclusión, el lugar de conteo realiza las operaciones de conteo de fallos y la suma de tiempos de fallo del componente diagnosticado.

La RdPD evalúa posibles cambios en cada rama. El diagnosticador emerge en funcionamiento normal o de fallo acorde a la función LA. El diagnostica- 
dor evalúa cada fallo separadamente y tiene en cuenta los fallos que son causados por otros fallos en sus transiciones, por lo tanto, es capaz de detectar fallos simultáneamente, correspondiente al orden en que ellos aparecen. Esta estructura de diagnosticador permite hacer la extensión al diagnóstico de fallos intermitentes. El diagnosticador presenta un gran aporte en el mantenimiento preventivo, a través del lugar de conteo, realiza la suma de los tiempos de fallo en que dura un fallo intermitente y las veces que sucede un fallo, con estos datos de tiempo y número de veces que ha fallado un dispositivo, se puede llevar a cabo la determinación del patrón de referencia de fallo, fundamental en los análisis del mantenimiento preventivo de los componentes, ya que se pueden determinar, no solo la evolución de los fallos, si no también su estado previo, buscando el ser reemplazado antes de fallo definitivo.

\subsubsection{Diagnosticabilidad}

En un sistema representado por una RdP, la diagnosticabilidad esta dada por la capacidad de poder identificar cuando el proceso cae en fallo. Por lo tanto, se dice que un sistemas es diagnosticable, si es posible detectar con un retardo finito, sucesos de transiciones de fallo de cualquier tipo, utilizando el registro de transiciones observadas [77].

Diagnosticabilidad: Una RdP $Q$ que representa el sistema, es diagnosticable con respecto a la distribución de fallos $\Pi_{f}$ sobre la $t_{f}$ si se cumple lo siguiente:

$$
\begin{gathered}
{\left[\forall S \in \mathcal{L}\left(Q, m_{0}\right), \wedge, S \in T_{o}\right]\left(\forall i \in \Pi_{f}\right)\left(\exists n_{i} \in \mathbb{N}\right)} \\
(\forall t \in L / S)\left[\|T\| \leq n_{i} \Rightarrow D\right]
\end{gathered}
$$

Sea $S$ una secuencia de transiciones observables y que pertenecen al conjunto de todas las secuencias de disparo alcanzables, desde el marcado inicial $m_{0}$, y $T$ cualquier continuación suficientemente larga de $S$. La condición de diagnosticabilidad $D$ requiere que cada secuencia perteneciente al conjunto de todas las secuencias de disparo que produce las transiciones observables $S T$, debe contener una transición de fallo del conjunto $T_{f}$. Esto implica que a lo largo de cada continuación $T$ de $S$ se puede detectar la aparición de un fallo del tipo $F_{i}$ con un retardo finito, específicamente a lo sumo en $n_{i}$ transiciones del 
sistema después de $S$.

En un sistema se pueden presentar dos diferentes tipos de fallos, que son: fallos normales o fallos críticos, un fallo normal se puede definir como aquel que afecta el funcionamiento del sistema, pero no afecta la disponibilidad del mismo. Un fallo crítico, por lo tanto, conduce al paro total del sistema. Entonces, Se adiciona al concepto de diagnosticabilidad enunciado anteriormente, que un sistema es diagnosticable cuando permite identificar no solo fallos normales si no también, puede definir cuando un fallo crítico ha ocurrido.

Un fallo crítico $F_{C}$, es aquel fallo que pertenece a la distribución de fallos de sistema, tal que, cuando en la RdP que representa el sistema, alcanza el marcado de fallo crítico, el sistema entra en estado crítico o de fallo total.

$$
\exists F_{C} \in \prod f \rightarrow M\left(p_{f c}\right)
$$

Una RdP que representa el modelo de un sistema, es diagnosticable si en un número finito de transiciones observables, alcanza un marcado de fallo $M\left(p_{f}\right)$, donde el $M\left(p_{f i}\right)$, solo o en unión con otro marcado de fallo $M\left(p_{f k}\right)$, permiten identificar un fallo de orden superior o crítico.

$$
\begin{gathered}
\left(S \in \mathcal{L}\left(Q, m_{0}\right), \wedge, S \in T_{o}\right),\left(\forall i, k \in \Pi_{f}\right)[\|S\|<\propto]: \\
M\left(p_{f i}\right)+M\left(p_{f k}\right) \rightarrow M\left(p_{f c}\right)
\end{gathered}
$$

En general, el diagnosticador es una RdP implementada tomando como punto de partida el modelo general refinado del sistema, realiza una observación on-line del modelo, con objeto de evaluar el comportamiento del sistema, también se puede saber si existen condiciones de diagnosticabilidad en el sistema, es decir se pueda alcanzar cualquier marcado de fallo $M\left(p_{f}\right)$.

\subsection{Ejemplo de Aplicación, Sistema de Envasado de Líquidos}

El ejemplo de sistema híbrido elegido es el sistema de envasado de líquidos tratado en el la sección 5.3. El sistema esta compuesto de dos partes: Una parte exclusivamente discreta y la parte que maneja tres variables continuas (nivel, $\mathrm{pH}$ y temperatura). La parte discreta ya se hizo el diagnóstico de fallos en el ejemplo del capítulo 5, esa parte será unida a la parte continua permitiendo 


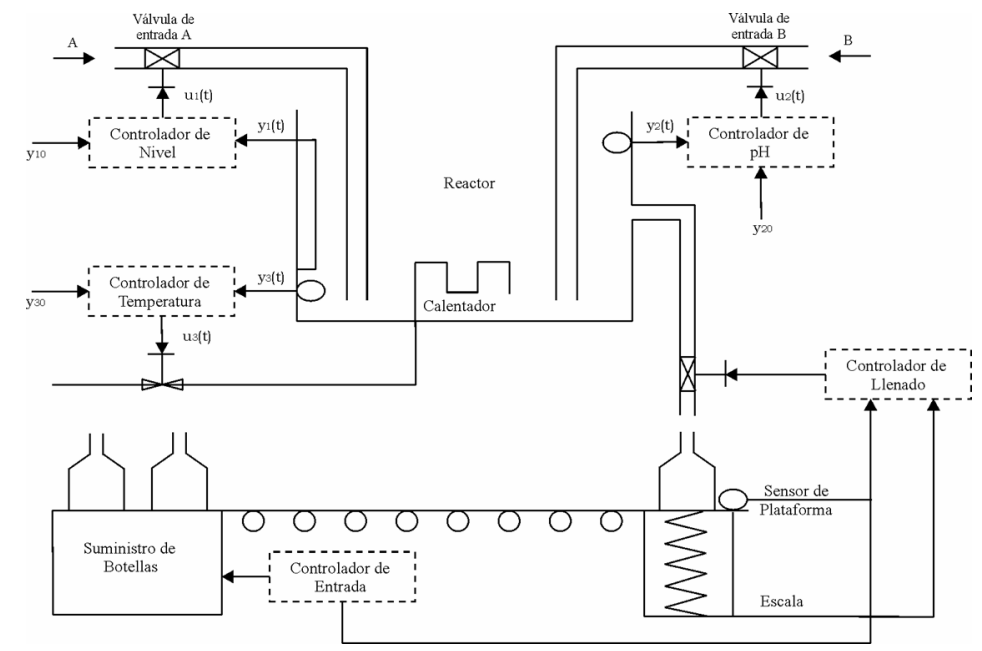

Figura 6.1: Sistema de Envasado de Líquidos- Híbrido

realizar el diagnóstico de fallos de un sistema Híbrido.

\subsubsection{Modelamiento continuo del Sistema de Envasado}

Antes de entrar a construir el modelo y diagnosticador del sistema de envasado, se presenta el modelamiento matemático que representa la dinámica continua del proceso dado en la figura 6.1. Para el sistema de nivel se dan las siguientes variables:

$\bar{Q}$ velocidad del flujo en estado estable, $\mathrm{m}^{3} / S g$

$q_{i}$ desviación pequeña de la velocidad de entrada, $\mathrm{m}^{3} / \mathrm{Sg}$

$q_{o}$ desviación pequeña de la velocidad de salida, $\mathrm{m}^{3} / \mathrm{Sg}$

$\bar{H}$ altura en estado estable, $m$

$h$ desviación pequeña de la altura a partir de su valor estable, $m$

El sistema se considera lineal, por lo tanto, el flujo es laminar. El flujo de entrada menos el flujo de salida durante un pequeño intervalo de tiempo $d t$, es igual a la cantidad adicional almacenada en el tanque, se observa que:

$$
C d h=\left(q_{i}-q_{0}\right) d t
$$


La relación de resistencia en el sistema esta dado por

$$
q_{0}=\frac{h}{R}
$$

La ecuación diferencial para el sistema con un valor constante de resistencia es

$$
R C \frac{d h}{d t}+h=R q_{i}
$$

$R C$ es la constante de tiempo del sistema. Aplicando la transformada de laplace y con condiciones iniciales en cero se obtiene

$$
(R C s+1) H(s)=R Q_{i}(s) \quad \text { donde } \quad H(s)=\mathcal{L}[h] \text { y } Q_{i}(s)=\mathcal{L}\left[q_{i}\right]
$$

Si $q_{i}$ se considera la entrada y $h$ la salida, la función de transferencia del sistema es

$$
\frac{H(s)}{Q_{i}(s)}=\frac{R}{R C s+1}
$$

No obstante, si $q_{0}$ se toma como la salida, y la entrada es la misma, la función de transferencia es

$$
\frac{Q_{0}(s)}{Q_{i}(s)}=\frac{1}{R C s+1}
$$

En el caso del modelamiento de la temperatura, asumiendo que la transferencia de calor se da por conducción, que el tanque está aislado para eliminar las perdidas de calor, que el líquido en el tanque esta perfectamente mezclado y que la temperatura que entra se mantiene constante se tiene que el flujo de calor $(\mathrm{Kcal} / \mathrm{Sg})$ es

$$
q=K \Delta \theta
$$

$\Delta \theta=$ diferencia de temperatura, ${ }^{\circ} \mathrm{C}$

$\mathrm{K}=$ Coeficiente, $\mathrm{Kcal} / \mathrm{sg}^{\circ} \mathrm{C}$

El coeficiente $K$ se obtiene mediante

$$
K=\frac{k A}{\Delta X}
$$

Donde

$k=$ conductividad térmica $\mathrm{Kcal} / \mathrm{m} \mathrm{Sg}{ }^{\circ} \mathrm{C}$

$A=$ área normal para flujo de calor, $m^{2}$ 
$\Delta X=$ espesor del conductor, $m$

$H=$ coeficiente de convección, $\mathrm{Kcal} / \mathrm{m}^{2} \mathrm{Sg}{ }^{\circ} \mathrm{C}$

La resistencia térmica para la transferencia de calor entre dos sustancias se define como

$$
R=\frac{d(\Delta \theta)}{d q}=\frac{1}{K}
$$

La capacitancia térmica $C$ se define mediante

$$
C=m c
$$

donde

$m=$ masa de sustancia considerada, $K g$

$c=$ calor específico de la sustancia, $\mathrm{Kcal} / \mathrm{Kg}{ }^{\circ} \mathrm{C}$

Sean

$\bar{\Theta}_{i}=$ temperatura en estado estable del líquido que entra, ${ }^{\circ} \mathrm{C}$

$\overline{\Theta_{0}}=$ temperatura en estado estable del líquido que sale, ${ }^{\circ} \mathrm{C}$

$G=$ velocidad del flujo del líquido, $\mathrm{Kg} / \mathrm{Sg}$

$M=$ masa del líquido en el tanque, $K g$

$c=$ calor específico del líquido, $\mathrm{Kcal} / \mathrm{Kg}{ }^{\circ} \mathrm{C}$

$R=$ resistencia térmica, ${ }^{\circ} \mathrm{C} \mathrm{Sg} / \mathrm{Kcal}$

$\mathrm{C}=$ capacitancia térmica, $\mathrm{Kcal} /{ }^{\circ} \mathrm{C}$

$H=$ entrada de flujo de calor, $\mathrm{Kcal} / \mathrm{Sg}$

Suponiendo que el flujo de calor de entrada al sistema (proporcionado por el calentador), cambia repentinamente de $\bar{H}$ a $\bar{H}+h_{i}$, donde $h_{i}$ representa un cambio pequeño en el flujo de calor de entrada. El flujo de calor de salida cambiará, entonces, de forma gradual, de $\bar{H}$ a $\bar{H}+h_{0}$. La temperatura del líquido que sale también cambiará de $\bar{\Theta}_{0}$ a $\bar{\Theta}_{0}+\theta$. Para este caso, $h_{0}, C$ y $R$ se obtienen, respectivamente, como

$$
\begin{gathered}
h_{0}=G c \theta \\
C=M c
\end{gathered}
$$




$$
R=\frac{\theta}{h_{0}}=\frac{1}{G c}
$$

La ecuación diferencial es

$$
C d \theta=\left(h_{i}-h_{0}\right) d t \quad o \quad C \frac{d \theta}{d t}=h_{i}-h_{0}
$$

la cual se puede escribir como

$$
R C \frac{d \theta}{d t}+\theta=R h_{i}
$$

La constante de tiempo del sistema es $R C$ segundos. La función de transferencia que relaciona $\theta$ con $h_{i}$ se obtiene como

$$
\frac{\bar{\Theta}(s)}{H_{i}(s)}=\frac{R}{R C s+1}
$$

\subsubsection{Aplicación del Proceso de Construcción del Diagnosticador}

A continuación se procede a aplicar el proceso para realizar el diagnóstico de fallos.

\section{Paso 1. Dividir el sistema en Subsistemas}

El sistema general es clasificado en cuatro partes que son: a) Subsistema discreto, b) Subsistema de temperatura, c) Subsistema de Ph, c) Subsistema de nivel. Cada subsistema está elegido por su independencia en el funcionamiento. ver figura 6.2 .

- Subsistema Discreto: Compuesto por el motor que hace girar la banda transportadora, la válvula de llenado, sensor de presencia de botella, sensor de llenado y controlador.

- Subsistema de Temperatura: Compuesto por el sensor de temperatura, válvula de inyección de presión de vapor y controlador de temperatura.

- Subsistema de pH (Potencial de Hidrógeno): Compuesto por el sensor de $\mathrm{pH}$, válvula de entrada $\mathrm{B}$ y controlador de $\mathrm{pH}$.

- Subsistema de Nivel: Compuesto por el sensor de nivel, válvula de entrada A y controlador de nivel. 


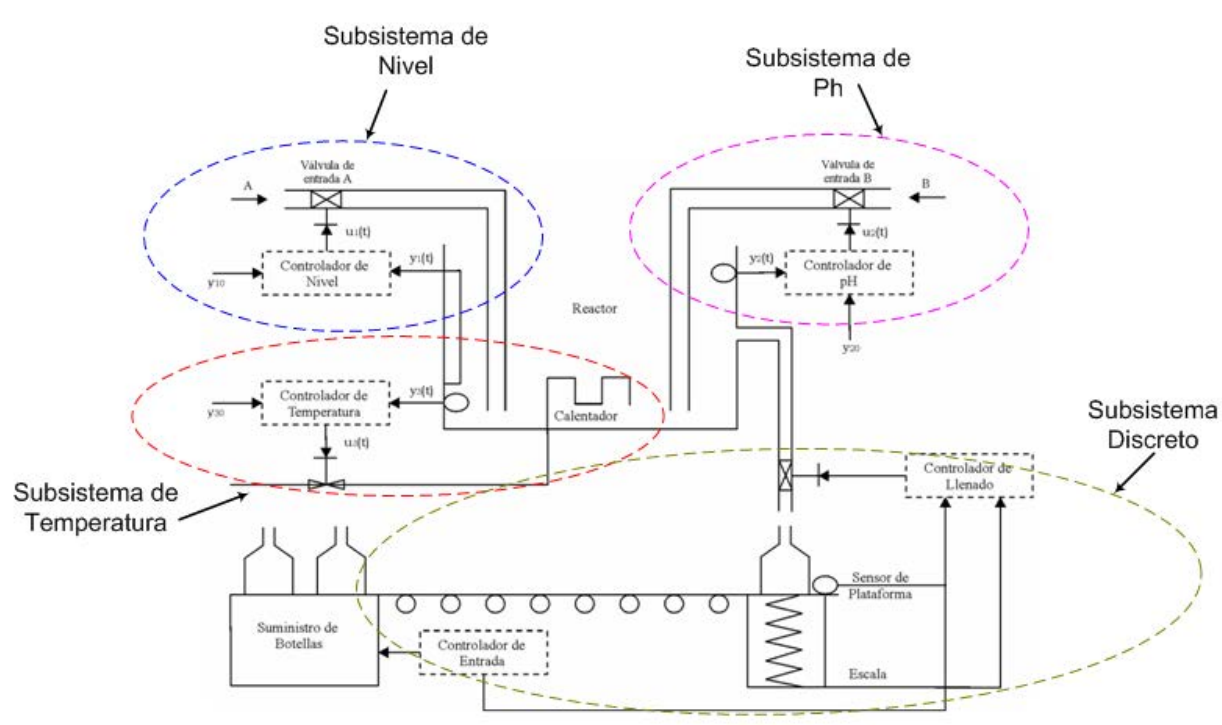

Figura 6.2: Subsistemas de Proceso de Envasado

Paso 2. Construir el modelo de RdP de los componentes de cada subsistema.

Se realiza el modelo de RdP de cada uno de los componentes de los subsistemas. En el modelo se incluye el comportamiento normal y de fallo.

1. Subsistema Discreto: El modelo fue construido en la sección 5.3, ver figura 5.6. En este subsistema se evaluarán los fallos de atascamiento (abierto y cerrado) $F 1$ y fallo de motor (en encendido y apagado) $F 2$.

2. Subsistema de Temperatura: Se diagnostican el fallo de válvula de inyección de vapor $F 3$. ver figura 6.3. La temperatura que se va a mantener en el líquido a envasar estará en un rango entre 60 y 90 grados celsius. Cuando la temperatura caiga por debajo del valor de 60 grados el calentador debe encender y cuando la temperatura sobrepase el valor de 90 grados, el calentador debe apagarse.

3. Subsistema de pH (Potencial de Hidrógeno): Se diagnostica el fallo de válvula de entrada $\mathrm{B} F 4$. ver figura 6.4. El rango de $\mathrm{pH}$ para el líquido a envasar puede estar entre 5 y 7 . Cuando el $\mathrm{pH}$ del líquido a envasar supere el nivel de 7 , la válvula de entrada $B$ se abrirá y cuando el $\mathrm{pH}$ esté por debajo de 5 la válvula se cerrará. 


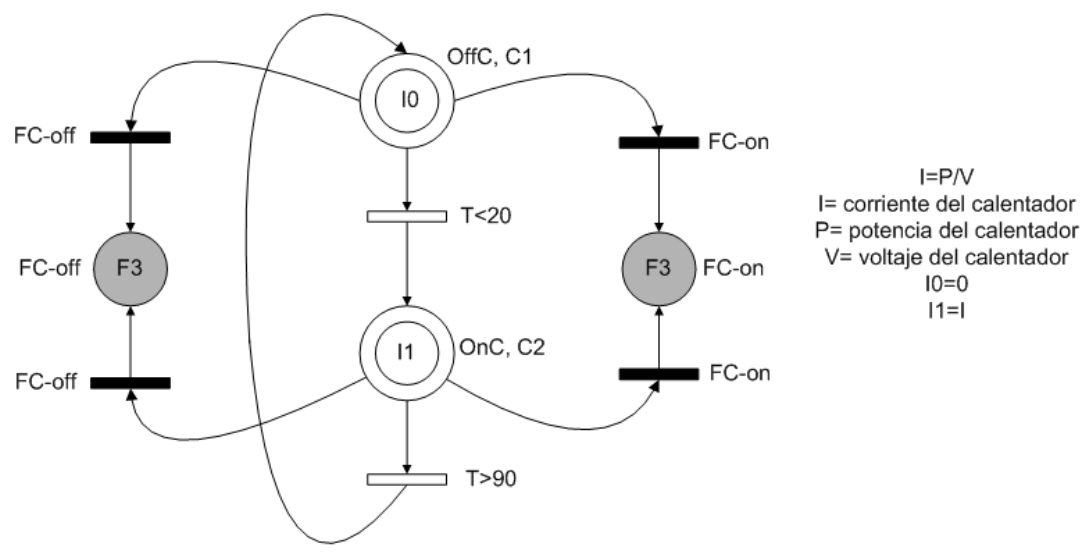

Figura 6.3: Modelo General de RdP de Subsistema de Temperatura

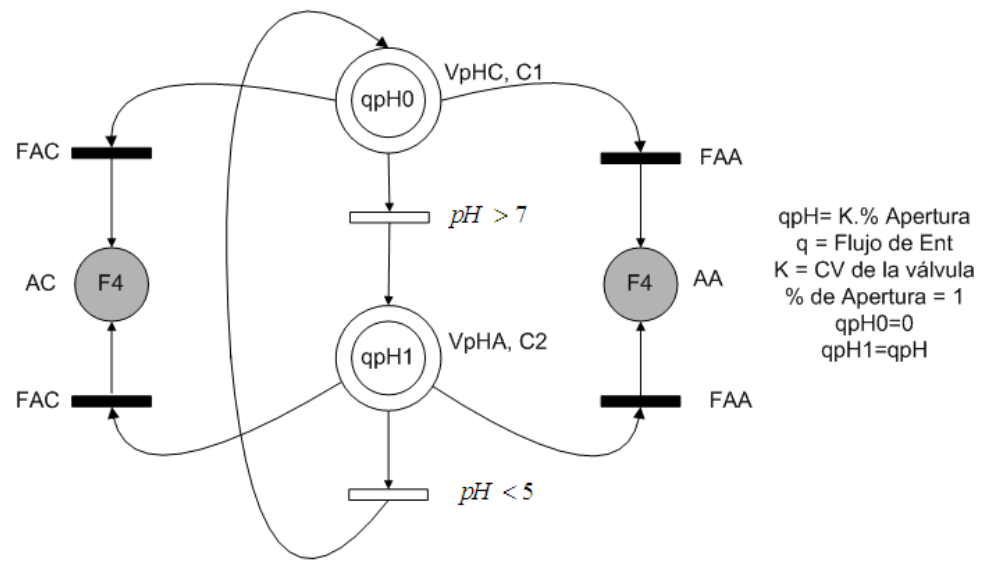

Figura 6.4: Modelo General de RdP de Subsistema de pH 


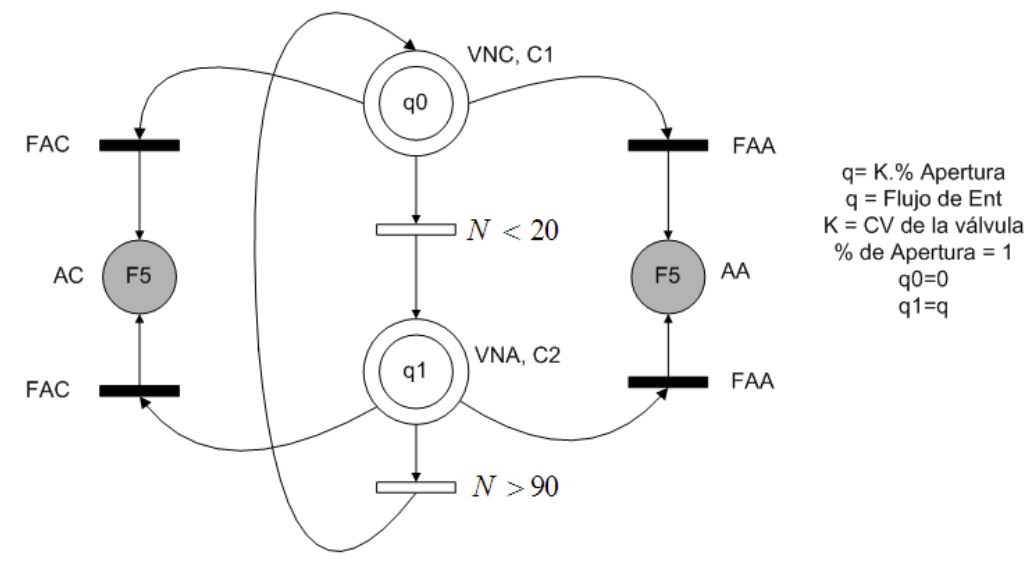

Figura 6.5: Modelo General de RdP de Subsistema de Nivel

4. Subsistema de Nivel: Se diagnostica el fallo de válvula de entrada A F5. ver figura 6.5. El rango de nivel del tanque puede estar entre 20 y 90 litros. La válvula de entrada $A$ se abrirá cuando el nivel del líquido esté inferior a 20 Lts y se cerrará cuando el nivel del líquido sea superior a 90 Lts.

Basado en que cada subsistema posee un solo componente, el mismo modelo de RdP cumple la función de modelo general y el paso siguiente (paso 3) de realizar la operación de integración es evitado.

Paso 4. Refinar el Modelo General. Se realiza el refinamiento de los modelos generales de los diferentes subsistemas. En el modelo general se percibe como si todos los subsistemas son discretos, pero vemos que cuando se reemplacen las transiciones no observables $t_{f}$, por las transiciones observables respectivas (lecturas sensoriales), algunas transiciones pasaran de ser discretas a ser lecturas continuas. El reemplazo mencionado anteriormente se basa en la tabla de integración de sensores construida previamente para cada subsistema y se descartan aquellas transiciones que no son alcanzables.

1. Subsistema Discreto: El modelo refinado fue construido en la sección 5.3, ver figura 5.8. La tabla de integración de sensores se puede observar en la tabla 5.4 .

2. Subsistema de Temperatura: En este subsistema la única lectura sensorial a tener en cuenta es la temperatura, por ello, no es necesario construir la tabla de integración de sensores. Por lo anterior, definimos que cuando la medida del sensor esté por fuera del rango de trabajo y dependiendo 


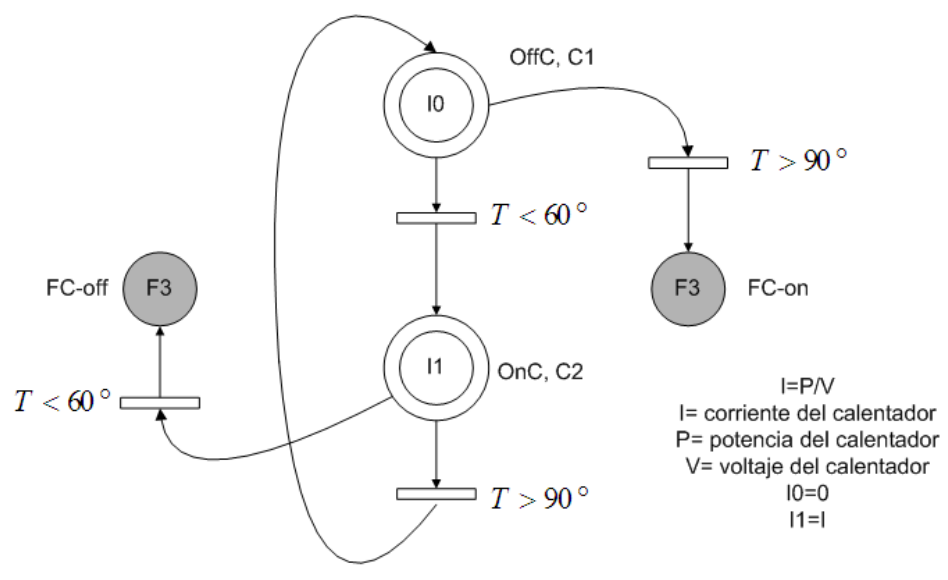

Figura 6.6: Modelo Refinado de RdP de Subsistema de Temperatura

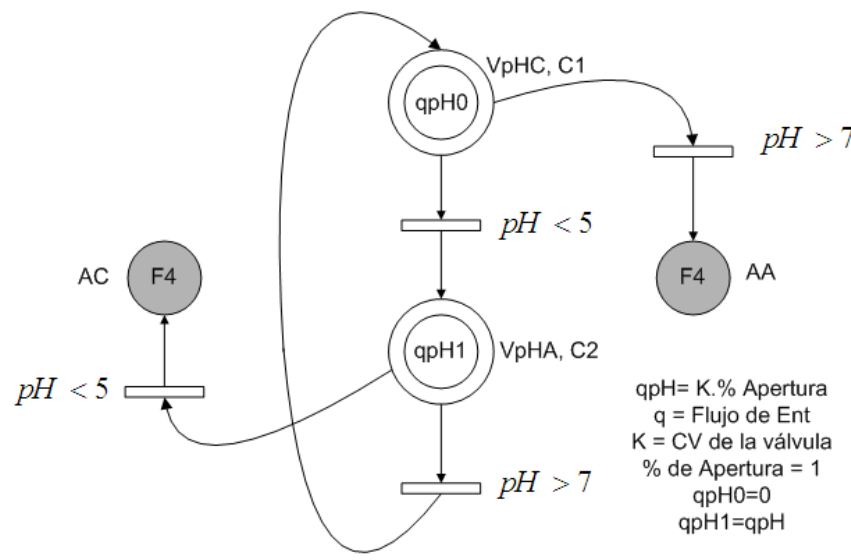

Figura 6.7: Modelo Refinado de RdP de Subsistema de pH

del lugar en que se encuentre el sistema, la medida del sensor nos puede determinar que existe un atascamiento de la válvula de inyección de vapor, el cual puede ser en abierto o en cerrado. Con las medidas del sensor definidas el modelo resultante será el de la figura 6.6.

3. Subsistema de pH (Potencial de Hidrógeno): Se presenta la única medida de $\mathrm{pH}$, se puede definir fallo de la válvula en el momento en que la lectura del sensor esté por fuera del rango, es decir, inferior a 5 y superior a 7 , teniendo en cuenta el estado del proceso. EL modelo refinado se puede observar en la figura 6.7 . 


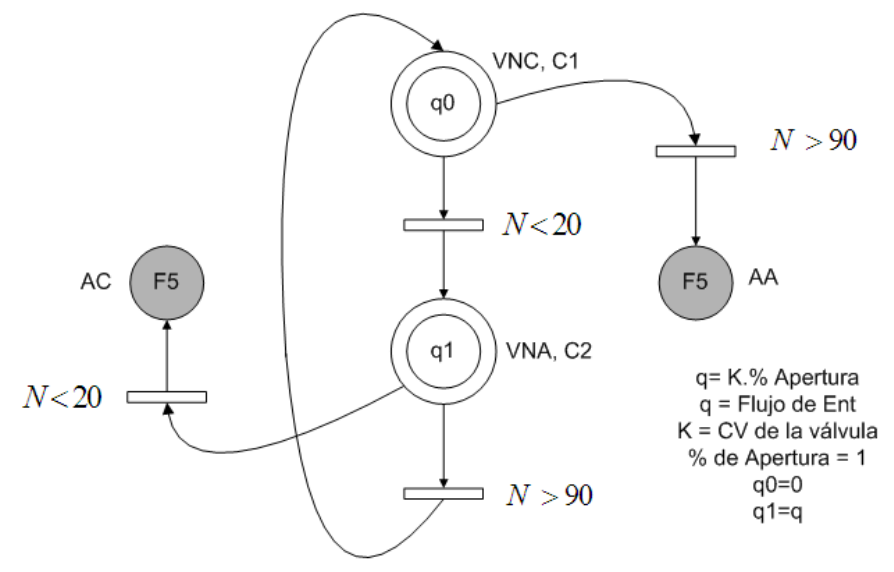

Figura 6.8: Modelo Refinado de RdP de Subsistema de Nivel

4. Subsistema de Nivel: Tenemos solo la medida continua de nivel, se presentará fallo cuando la medida esté por fuera del rango de 20 y 90 litros manteniendo la condición del estado del proceso. Con una medida inferior a 20 litros se presenta fallo de atascamiento en cerrado y superior a 90 litros el fallo es atascamiento en abierto. En la figura 6.8 se observa el modelo de RdP refinado del subsistema de nivel.

Paso 5 Construir el diagnosticador. Se construye el diagnosticador basado en las funciones de $A L$ y $E F$. Las etiquetas de fallo definidas y mencionadas anteriormente son: $F 1$ fallo de válvula de llenado $(A A, A C), F 2$ fallo de Motor (Mon, Moff), $F 3$ fallo de válvula de inyección de vapor, $F 4$ fallo de válvula de entrada $B$ y $F 5$ fallo de válvula de entrada $A$. El diagnosticador es el mismo para un sistema discreto, con la adición de tres nuevas ramas que diagnostican los subsistemas continuos. Así mismo, se percibe que el diagnosticador realiza un diagnóstico individual de los subsistemas, manteniendo las grandes ventajas mostradas en el capítulo 4. El diagnosticador se puede ver en la figura 6.9 . 


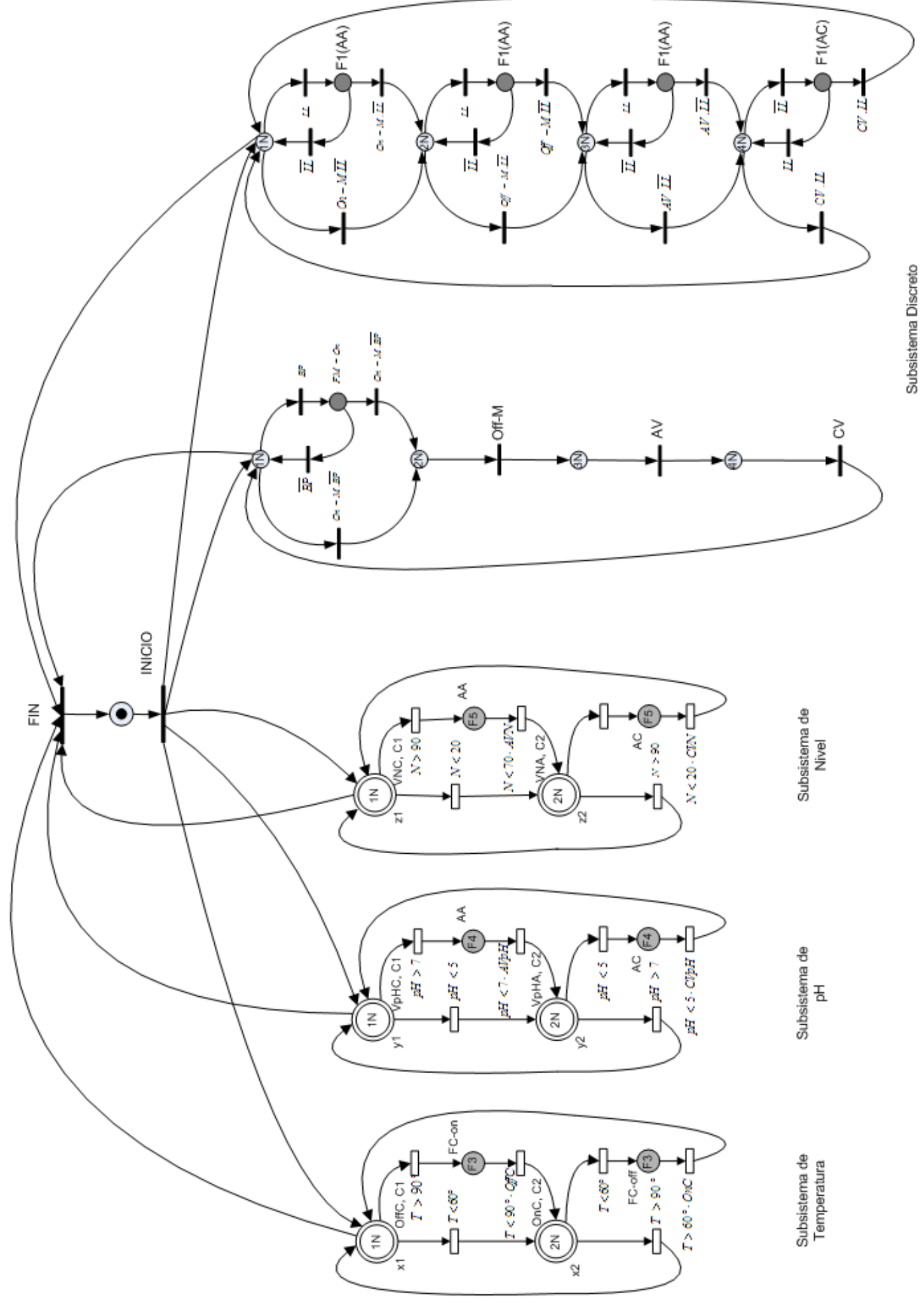

Figura 6.9: Diagnosticador Híbrido Sistema de Envasado 


\section{Capítulo 7}

\section{Implementación y Pruebas de Campo del Diagnosticador en el HC no Tripulado.}

\subsubsection{Introducción}

Actualmente, se ha evaluado la herramienta de manera teórica, sobre sistemas de manufactura (HVAC y línea de envasado), verificándose su validez y permitiendo evaluar las ventajas presentadas frente a otras alternativas desarrolladas por otros investigadores. Dado que el diseño del algoritmo es en gran medida independiente del proceso al que se aplica, se pretende en el desarrollo de esta tesis, aplicar la metodología al diagnóstico de fallos en los sistemas de actuación, navegación y control de robots aéreos, donde se encuentra una buena plataforma de sistema complejo e híbrido.

La historia de los accidentes de las aeronaves tripuladas es muy antigua, acorde a las estadísticas de la oficina de Registro de Accidentes Aéreos de Génova Suiza, un total de 121,870 personas han muerto en 17,369 accidentes en 100 años de la historia de la aviación (1905-2005)[48]. Las principales causas de estos accidentes fueron clasificados como errores humanos, fallos técnicos, clima y sabotaje. Destacar que los errores humano son responsables del $67.57 \%$ de estos accidentes, el cual es bastante alto comparado con otras causas de accidentes. Los avances en tecnología llevan al desarrollo de los vehículos aéreos no Tripulados (UAV), ofreciendo considerables ventajas. El concepto 
CAPÍTULO 7. IMPLEMENTACIÓN Y PRUEBAS DE CAMPO DEL DIAGNOSTICADOR EN EL HC NO TRIPULADO.

de la eliminación de las limitaciones mental y física humana dan nacimiento a la tecnología no tripulada. Se asumió que menos implicación del hombre debería reducir las oportunidades de fallo debido a errores humanos. Sin embargo, esta suposición ha sido descartada, dado que las estadísticas muestran un mayor número de accidentes en sistemas no tripulados debido a error humano, comparado con las aeronaves tripuladas. Muchos de los accidentes ocurridos durante las fases de despegue y aterrizaje de los vuelos, envuelven la participación humana en sistemas no tripulados. [3] Realiza un estudio para determinar factores casuales en accidentes de UAV's, usa una muestra de 56 accidentes de UAV's de la Armada de los Estados Unidos. El estudio reveló que el factor humano está envuelto en 18 accidentes (32\%). Esto le permite recomendar que la interfase hombre maquina en la tecnología UAV debe mejorar, y que la habilidad de la tripulación del UAV para ajustarse en diferentes escenarios debe ser ampliada a través de entrenamiento extensivo.

Los vehículos aéreos no tripulados (UAV's), ya sean totalmente autónomos o teledirigidos, son de gran importancia en el mundo actual, debido a que permiten aislar la intervención humana en operaciones que representan riesgos para el piloto. Los grandes esfuerzos en desarrollo en los UAS, han sido enfocados solo en el control automático de la aeronave, dejando de lado la seguridad de la misma. Los UAV civiles, debido a su pequeño tamaño y escasa robustez, presentan gran vulnerabilidad y relativa poca fiabilidad en vuelo, ya sea por fallos presentados en sus diferentes componentes, o por condiciones ambientales adversas (viento, humedad, temperatura). Actualmente existe poca información documentada o escasas investigaciones dedicadas al estudio de las causas que conducen a fallos en la aeronave, siendo prácticamente imposible identificar los puntos críticos que puedan comprometer su misión. Algunos investigadores en UAS como [40] citan como problema fundamental la pérdida de comunicaciones entre la aeronave y la estación de control en tierra.

Por otro lado, de entre todas las plataformas habitualmente utilizadas, el helicóptero destaca como la plataforma más habitualmente usada para crear UAS, debido a su capacidad de realizar vuelos estacionarios, despegue y aterrizaje vertical, baja altitud, baja velocidad y flexibilidad en los vuelos. Al mismo tiempo posiblemente es la aeronave más vulnerable, debido a su complejidad mecáni- 
ca, lo que hace que sea muy elevado el número de variables a controlar en vuelo y que éstas estén fuertemente relacionadas entre sí. Por todo ello, se plantea como un requisito fundamental el hecho de prestar gran atención a la fiabilidad de la aeronave, ya que a medida que aumenta su grado de automatización, aumentan las posibilidades de fallos en su sistema y en la estación de control en tierra. Los helicópteros tienen un amplio rango de aplicaciones militares, pero también en el dominio Civil.

En caso de ocurrencia de fallos en sensores, actuadores o estructura de la aeronave, Los helicópteros no tienen las mismas propiedades de las aeronaves de ala fija. Especialmente un helicóptero no tripulado tiene tamaño pequeño, peso ligero, estructura compacta y no tiene redundancia de actuadores y sensores. Un Fallo en cualquier parte del UAV puede ser catastrófico. Si el fallo no es detectado y acomodado a tiempo, el HC se puede estrellar [45]. Las características estructurales, condiciones de uso y ambiente hacen que la rata de accidentes de HC sea mayor que las aeronaves de ala fija, en 2012, 160 accidentes de HC civiles ocurrieron en USA [48]

Los fallos en los UAV pueden ser clasificados acorde a su localización de ocurrencia

- Fallos en el actuador. Representa la pérdida parcial o total de la acción de control del actuador. Los fallos del actuador del UAV incluyen principalmente fallos de salida constante (servo atascado), fallos de cambio de ganancia constante (porcentaje del valor normal) y fallos a la deriva (drift).

- Fallos de los sensores. Representan lecturas incorrectas de las medidas con que el UAV está equipado. Principalmente incluyen fallos total, fallos de desviaciones constantes (constant bias), fallos de ganancia constante y fallos atípicas (outlier).

- Fallo de componentes. Representa fallos en los componentes de la estructura misma. Este fallo representa cambios en los parámetros físicos del $\mathrm{HC}$, como la pérdida del a cola del HC y parte de la pérdida del rotor de cola [34].

En el presente capítulo se describe la aplicación de la herramienta de diagnóstico de fallos para sistemas híbridos basados en Redes de Petri, el cual será eva- 
luado en un helicóptero de radio-control como vehículo base. La herramienta de diagnóstico de fallos diseñado, requiere ser alimentado con datos reales de campo. Estos datos provienen del muestreo de las variables que se consideren críticas del sistema en evaluación, por ello en primer lugar se han identificado y medido las variables mas susceptibles de fallar en el helicóptero, y que permiten analizar y detectar las causas que conllevan a abortar una misión o a la perdida de la aeronave. Así pues, el proceso de diagnóstico de fallos es una herramienta útil para reducir los costosos accidentes que se ven reflejados en pérdidas importantes de dinero y tiempo.

En el proceso de adquirir las señales de campo, ubicadas en el helicóptero, se ha diseñado y construido todo el hardware necesario para capturar y enviar la información en tiempo real de cada variable, desde el helicóptero a una estación de control en tierra, la señales son encapsuladas y enviadas por un medio inhalámbrico a una frecuencia de $2.4 \mathrm{Ghz}$, esta información debe ser almacenada tanto en el mismo helicóptero como en el computador de la estación de control, la cual servirá para alimentar la herramienta de diagnóstico de fallos y definir el estado de la aeronave. En este mismo capítulo se hace una descripción de los componentes del helicóptero de radio control elegido para la investigación, su funcionamiento y las variables críticas a monitorizar, que se consideran como indicadores de riesgo durante las misiones; descripción de la construcción y características de los módulos de captura de las señales de campo, descripción de la aplicación de software (implementado en LabVIEW), que cumple la función de supervisor, a través del cual, se pueden visualizar las señales y enviar a archivos donde se almacene la información recogida en las misiones y por último, se realiza el análisis de resultados que resultan de la aplicación de la herramienta al HC volando en condiciones normal y de fallo.

\subsubsection{El Helicóptero no Tripulado y el Sistema de Adquisición de Datos}

Se entiende por aeronave no tripulada (UAV: Unmanned Aerial vehicle o también UAS: Unmanned Aerial System o UAVS: Unmanned Aerial Vehicle System), a aquella que es capaz de realizar una misión sin necesidad de tener una tripulación embarcada. Debe entenderse que esta condición no excluye la existencia de piloto, controlador de la misión u otros operadores, que pueden realizar su trabajo desde tierra. La extensión de vehículo a sistema, refleja que 


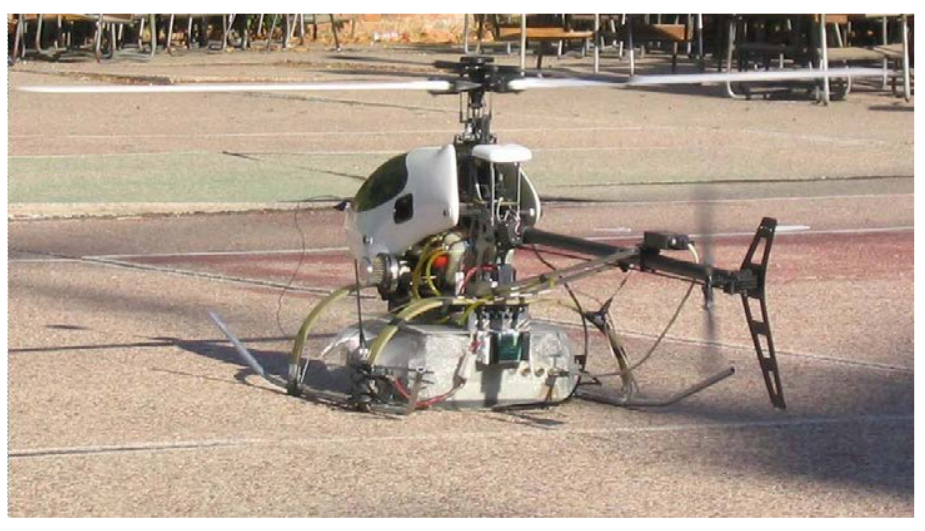

Figura 7.1: Helicóptero Vario UAV

el UAV precisa, no solo de la aeronave adecuadamente instrumentada, si no también de una estación en tierra, que complementa la instrumentación y capacidades embarcadas, ver figura 7.1.

Desde hace algunas décadas las aeronaves no tripuladas han sido motivo de interés, en particular en el ámbito militar, que han pasado de sistemas experimentales a equipos aptos para su uso profesional. Las posibilidades de crecimiento e impulso a la investigación en el uso de UAV en actividades no militares son notables. Junto al interés en aplicaciones militares, la ampliación de su uso a misiones civiles ha originado la aparición de un número apreciable de grupos de investigación y de pequeñas empresas dedicados al desarrollo de los subsistemas, a la integración de los mismos o a la puesta en marcha de aplicaciones y servicios basados en el uso de aeronaves no tripuladas.

Aplicaciones civiles para los UAV's existen en diversas áreas tales como: Patrulla de fronteras y costas, obtención de datos para cartografía, lucha contra incendios, monitorización de infraestructura energética. Apoyo a agentes de la ley, búsqueda y rescate, control de tráfico marítimo, supervisión de materiales peligrosos, gestión de crisis, entre otros.

En la actualidad existe una nueva regulación de las autoridades acerca del uso de las UAVs, pero debido al gran incremento de sus operaciones, se hace necesaria la implementación de unas recomendaciones que definan su uso y clasificación, buscando evitar poner en riesgo las personas, áreas de vuelo, y no ocupar el espacio de las aeronaves tripuladas. Una única fuente de información acerca de la fiabilidad de los UAVs está en el campo militar [68]. Basados en esta necesidad, a comienzos del año 2009 como grupo de investigación 
CAPÍTULO 7. IMPLEMENTACIÓN Y PRUEBAS DE CAMPO DEL DIAGNOSTICADOR EN EL HC NO TRIPULADO.

contactamos a la organización UVS International, con el objetivo de iniciar una recopilación de información que nos permitiese conocer los problemas mas comunes que se presentan en las misiones con las aeronaves, alrededor de la Unión Europea, en primera instancia fuimos recibidos con entusiasmo y nos dieron el visto bueno para elaborar a través de ellos la encuesta a los grupos afiliados a la organización, ver apéndice $A$, Enviamos la encuesta y creemos que por tramites burocráticos no se llevo a cabo la misma. la cual hubiese sido un gran aporte no solo a conocer los fallos en primera instancia, si no también, el inicio de la regulación de las autoridades sobre la utilización de UAVs que crece a pasos agigantados.

A continuación se describe la aeronave utilizada y el sistema de adquisición de datos, diseñado para poder implementar la herramienta de diagnóstico de fallos.

\section{Helicóptero No Tripulado}

Para la aplicación en particular del algoritmo de FD, Un helicóptero de radio control (no tripulado) modelo Vario Benzin Trainer fue elegido para este estudio, para conocer sus características mas importantes ver la tabla 7.1, el cual ha servido de herramienta de aplicaciones en una variedad de investigaciones sobre control autónomo en UAVs, en el grupo de investigación de Robótica y Cibernética de la Universidad Politécnica de Madrid [4]. El sistema (Helicóptero) está compuesto de tres subsistemas principales: Motor, rotor principal (conocido como plato) y rotor de cola, ver figura 7.2. Si cualquiera de ellos falla, la misión inevitablemente tiene que ser abortada, porque el helicóptero podría estrellarse con serias consecuencias rápidamente.

El sistema motor se compone de un pequeño motor de gasolina y un servo que controla el acelerador, ver figura 7.3. Es responsable de generar la rotación de las palas, para el rotor principal y de cola, en una relación proporcional asentada mecánicamente. Un controlador electrónico se utiliza normalmente para mantener la velocidad constante de rotor, independientemente del ángulo de ataque de las palas. Este ángulo está directamente relacionada con la resistencia que el motor tiene que superar..

El sistema de rotor principal está controlado por cuatro servos, ver figura 7.4. Permiten modificar la elevación y actitud del helicóptero (es decir, los ángulos de 


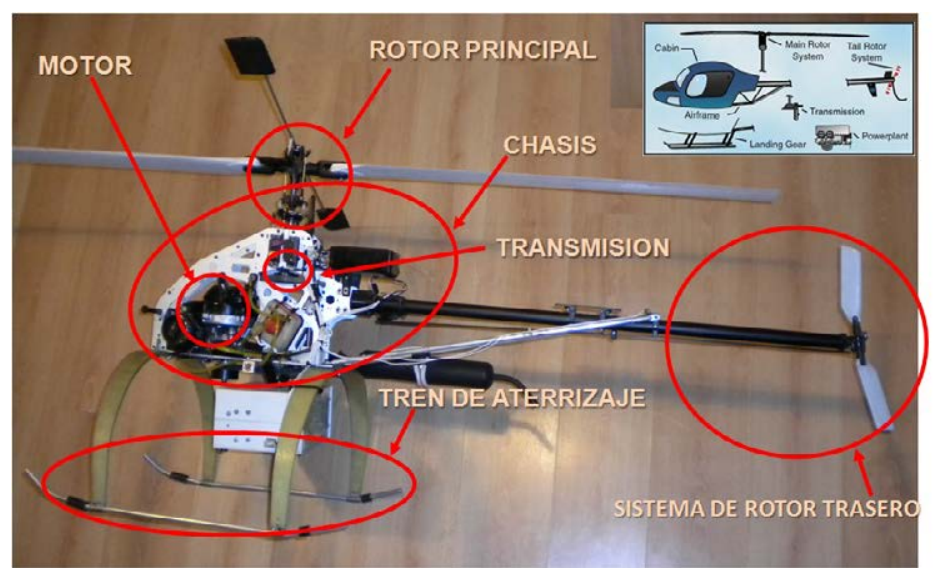

Figura 7.2: Sistemas que Componen un Helicóptero

Tabla 7.1: Características del Helicóptero Robbe

\begin{tabular}{|c|c|}
\hline \multicolumn{2}{|c|}{ CARACTERÍSTICAS DEL HELICÓPTERO VARIO } \\
\hline \hline Fabricante & VARIO \\
\hline Largo & $1460 \mathrm{~mm}$ \\
\hline Ancho & $200 \mathrm{~mm}$ \\
\hline Alto & $520 \mathrm{~mm}$ \\
\hline Diametro del Disco & $178 \mathrm{~mm}$ \\
\hline Motor & $23 \mathrm{cc}$ \\
\hline Peso Total & $7,3 \mathrm{Kg}$ \\
\hline Capac de peso de Levant. & $10.0 \mathrm{Kg}$ \\
\hline Relación de Transmisión & $9: 1: 1$ \\
\hline Control & 6 servos \\
\hline
\end{tabular}

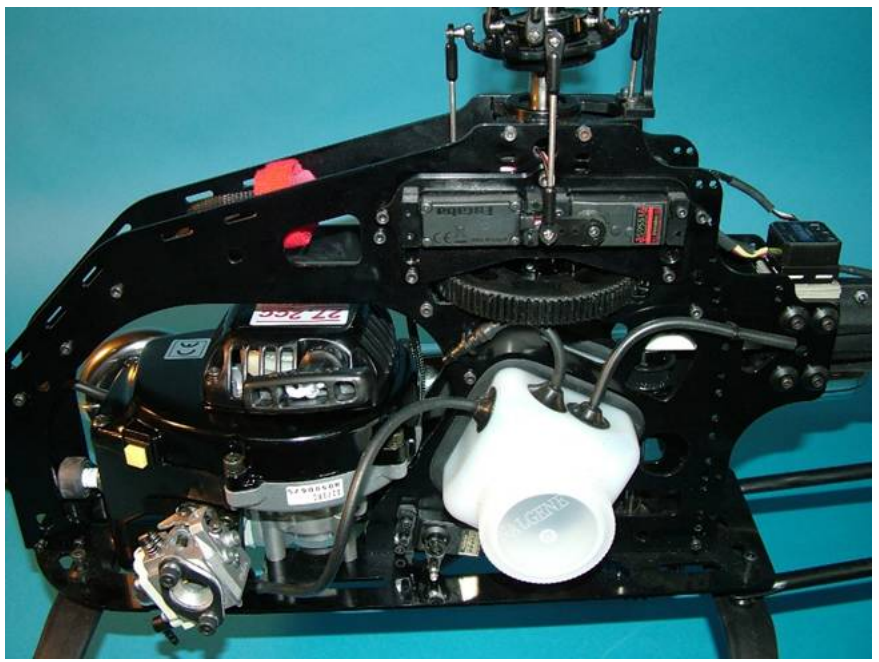

Figura 7.3: Motor de Combustión del Helicóptero Vario Benzin Trainer. 


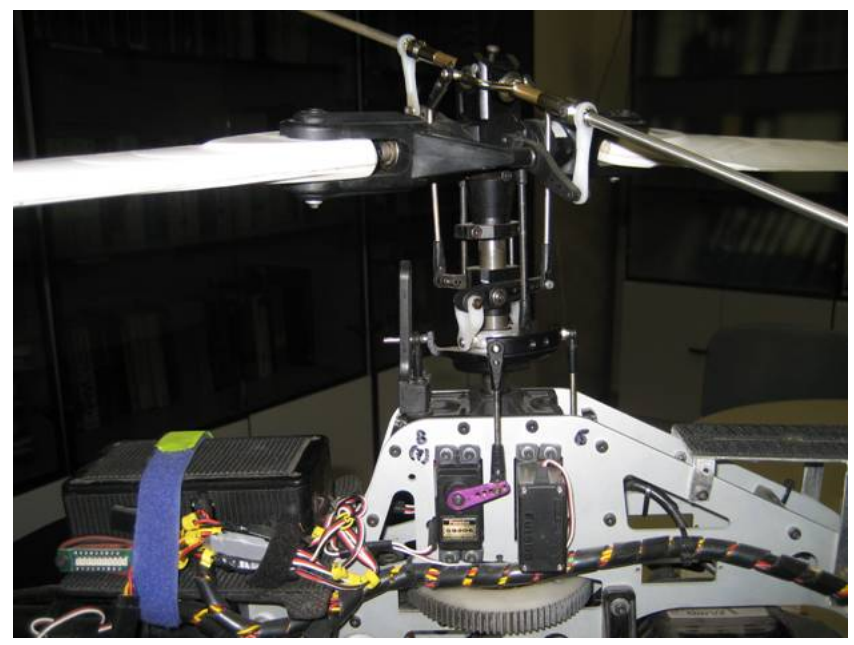

Figura 7.4: Sistema de Rotor Principal

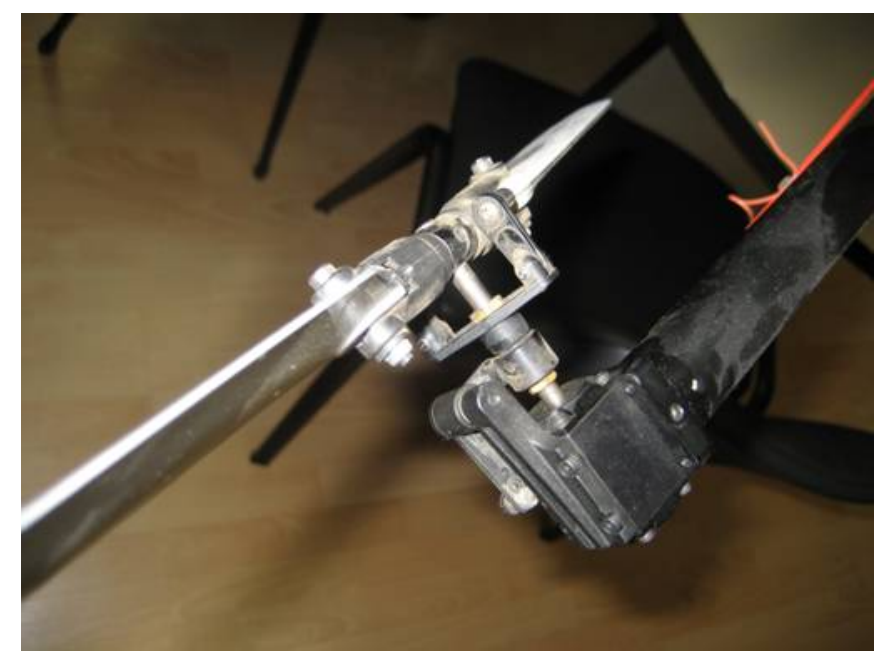

Figura 7.5: Sistema del Rotor de Cola.

balanceo y cabeceo) variando el paso colectivo y cíclico de las palas a lo largo de su rotación. Así, el paso colectivo varía la elevación y el cíclico modifica la actitud.

El componente principal del sistema de rotor de cola (anti-torque) es un controlador de servo, ver figura 7.5, que recibe comandos desde el piloto con el fin de modificar el ángulo de paso de las palas del rotor de cola. La variación de este ángulo permite modificar el ángulo de guiñada del helicóptero (cabeceo), principalmente porque varía el anti-torque que se requiere para compensar el torque generado por el rotor principal. 
El sistema completo se apoya en los dispositivos adicionales, como la fuente de alimentación, sensores de navegación (es decir, los sistemas inerciales, giróscopo de cola, GPS y compás electrónico), controladores, comunicaciones (antenas y radios), y la estación de control de tierra.

\section{Sistema de Adquisición de Datos.}

Un completo sistema de adquisición de datos (DAQ) se ha diseñado y construido para implementar el diagnosticador. Está compuesto por dos principales componentes: la instrumentación embarcada y la instrumentación en la estación de control, incluida está la interfaz gráfica (construida en LabVIEW), que es responsable de presentar los datos y de albergar a la Red de Petri Diagnosticadora. El sistema DAQ es responsable de medir las variables de campo del helicóptero y enviar la información a la estación de control en tierra. Esta información es usada para alimentar la herramienta de diagnóstico de fallos; el cual monitorea la operación normal y de fallo de la aeronave usando la RdPD. Varias variables fueron elegidas para monitorear el sistema completo acorde a la experiencia de los grupos de investigación y las recomendaciones de pilotos expertos, es decir:

- Temperatura del motor $(T)$

- Voltaje de Batería $(V)$

- Flujo de Combustible $(F)$

- Revoluciones por Minuto del Motor $(R P M)$

- Vibraciones del Chasis $(G)$

- Corriente (Is) de cada servo que permite controlar la aeronave (es decir, acelerador, cuatro para el plato y el de cola)

- Orientación (ángulos roll $(R l)$, pitch $(P t)$ y yaw $(Y w)$ )

- Ubicación (Latitud y longitud).

En la figura 7.6 se representa el esquema general de adquisición de datos. Sobre la aeronave son instalados los diferentes sensores, cada modulo de sensado envía la información a un ordenador central basado en microcontrolador. 
Una vez los datos son recogidos, estos son enviados por radio a la estación de control usando un sistema de comunicación XBee. La información es también almacenada a bordo dentro de una tarjeta SD (concepto de caja negra). EI computador en la estación de control recibe la información a través del puerto USB en orden a mostrar datos en la pantalla al piloto usando una aplicación desarrollada en LabVIEW.

Actualmente, el diagnosticador funciona en el computador de la estación de control en tierra en ordena maximizar la flexibilidad del sistema durante las pruebas. Sin embargo, se ha planeado implementar en un modulo CompactRIO buscando reducir el ancho de banda requerido e incrementar la frecuencia de adquisición. De esta manera, solo fallos y señales de alarma deberían ser enviados a la estación de control.

Toda la demás información (es decir, número de fallos, tiempos de fallos, variables leídas, etc) serán usadas en el trabajo posterior de mantenimiento preventivo y predictivo de la aeronave. Una breve descripción de los principales componentes del sistema de adquisición de datos son presentados a continuación:

- Temperatura. Evalúa la temperatura del motor, rango desde $-50^{\circ} \mathrm{C}$ a $150^{\circ} \mathrm{C}$. el sensor utilizado es el ADS22100, ver figura 7.7.

- Voltaje de Batería. Se evalúa directamente en la entrada análoga midiendo la tensión en la batería.

- Revoluciones por minuto. Se mide a través del sensor de efecto hall UGN3503. ver figura 7.8 .

- Flujo de combustible. Se utiliza el sensor FHK, con un rango de medida de 0,041 a $6 \mathrm{~L} / \mathrm{min}$. ver figura 7.9 .

- Vibraciones. Se miden a través del acelerómetro DE-ACCM2G2, de éste se extrae la información de mínima amplitud. ver figura 7.10,

- Corriente de los servos. Se mide a a través del voltaje de alimentación que entrega el sensor de corriente y se mide en $\mathrm{mA}$, ver figura 7.11, se usa la misma configuración para los 4 servos del plato, el rotor de cola y el servo de gas. 


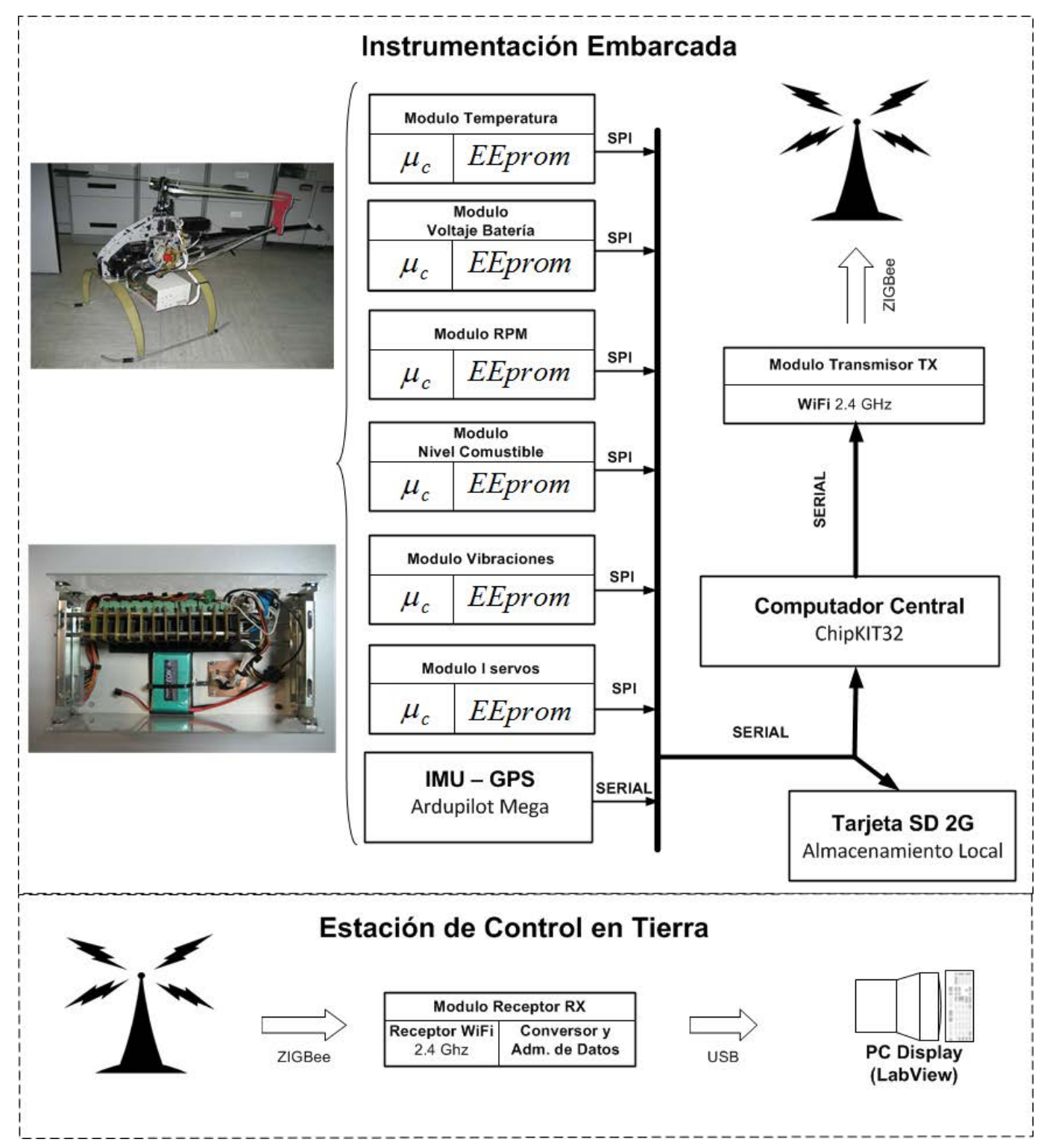

Figura 7.6: Esquema General de Adquisición de Datos HC 

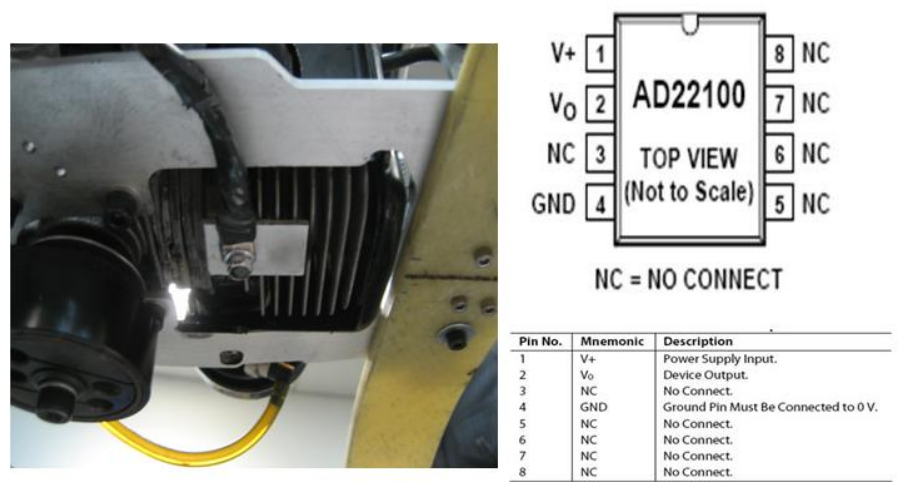

Figura 7.7: Sensor de Temperatura HC

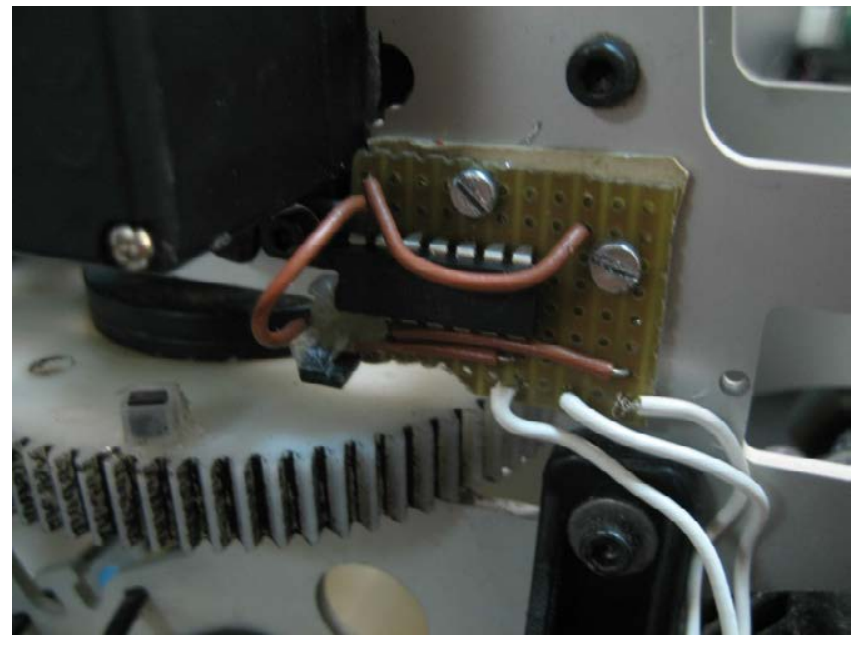

Figura 7.8: Sensor de Velocidad del Rotor

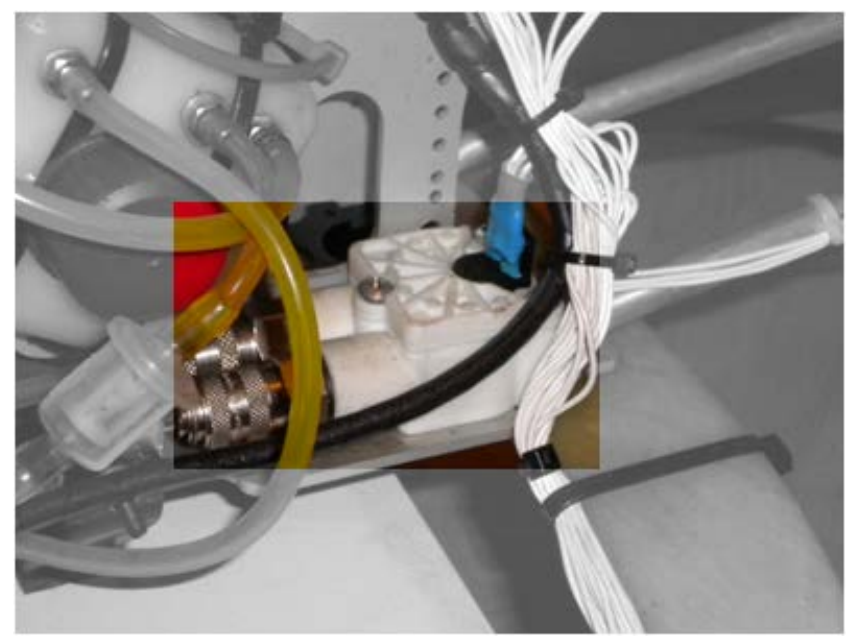

Figura 7.9: Sensor de combustible 


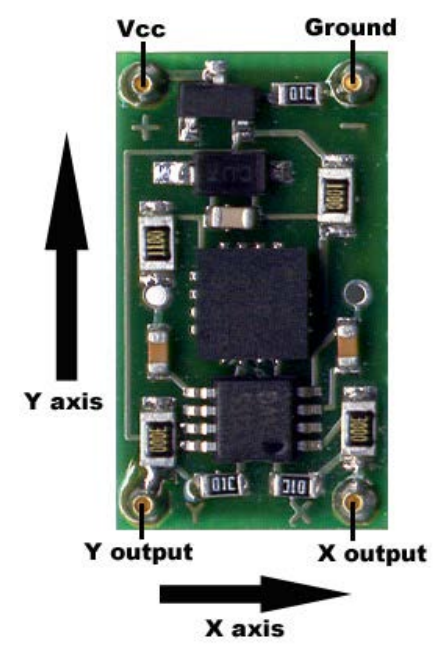

Figura 7.10: Sensor de Vibraciones HC
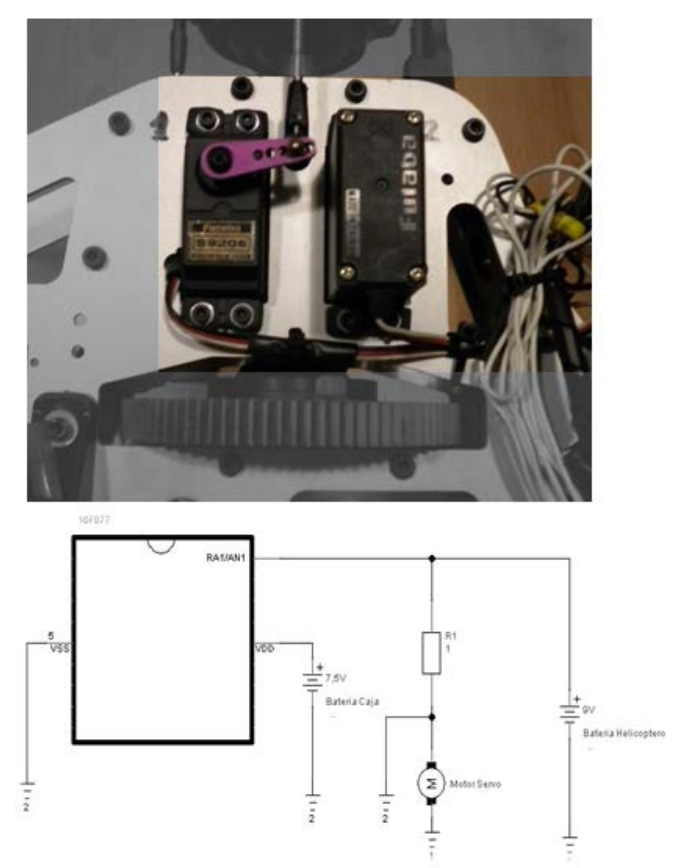

Figura 7.11: Sensor de Corriente de los Servos 


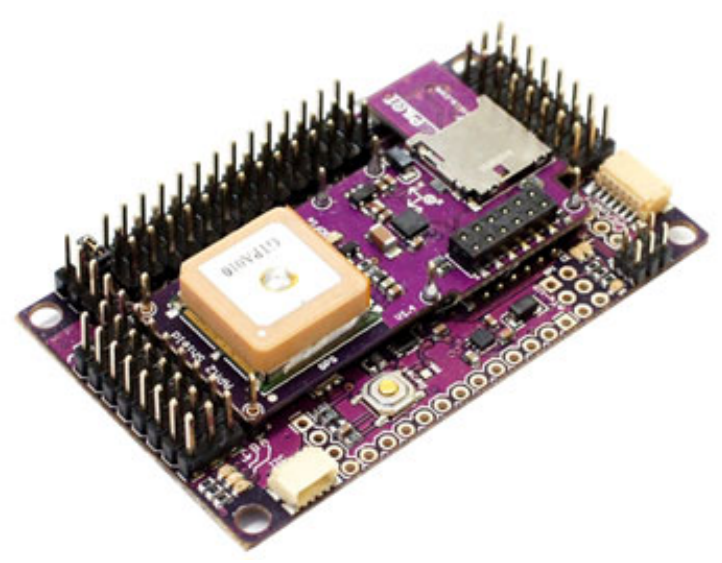

Figura 7.12: Sensor Orientación - Posición

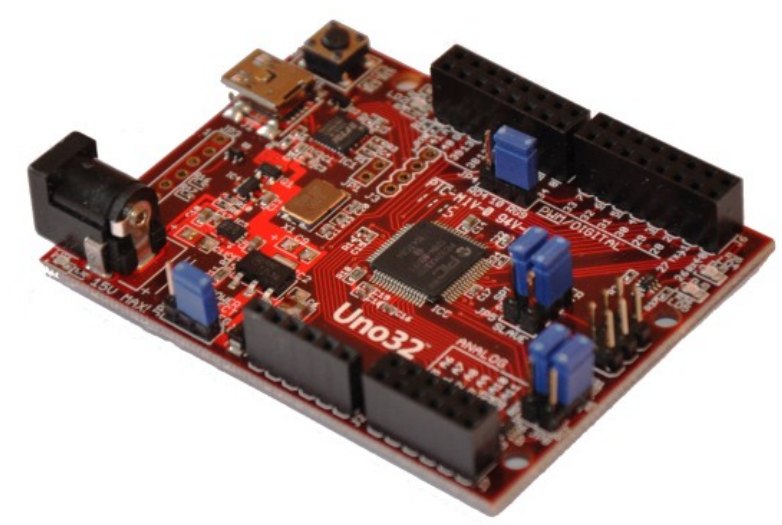

Figura 7.13: Computador Central ChipKit

- IMU - GPS. Ardupilot mega modelo 2.4 es el dispositivo el cual nos proporciona información de la orientación y posición de nuestra aeronave obtenida a través de su GPS integrado, tiene incorporados giróscopos, acelerómetros, magnetómetros, compás digital y sensor barométrico, ver figura 7.12 .

- Computador Central. Esta función la cumple el microcontrolador ChipKIT32, realiza la adquisición de datos provenientes desde la tarjeta Ardupilot y procesa la información proveniente de las otras unidades de sensado de variables físicas del helicóptero, ver figura 7.13,usa el protocolo de comunicación MAVLINK.

- Modulo de Transmisor-Receptor. Es la interfase inhalámbrica entre la aeronave y la estación de control en la banda de $2.4 \mathrm{GHz}$, utiliza el modulo 


\section{$4.9 \%$}

40

Figura 7.14: Modulo transmisor Receptor XBee

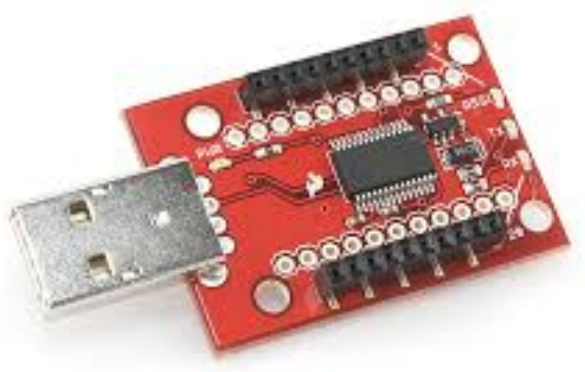

Figura 7.15: Modulo Conversor Serial-USB FTDI

XBee, ver figura 7.14. Un modulo FTDI (Future Technology Devices International) convierte la comunicación serial para introducirla en el computador via el puerto USB, ver figura 7.15.

\section{Aplicación de Software en LabVIEW}

Una Interfaz gráfica en LabVIEW se ha construido. Como mencionamos previamente, es responsable de adquirir, almacenar y presentar todos lo datos de las diferentes misiones del UAV. Como se puede ver en la figura 7.16, esta aplicación está distribuida en cuatro módulos, que son: descripción del proyecto, adquisición de datos, análisis y presentación de datos, y red de petri diagnosticadora.

El modulo de descripción del proyecto, muestra un pequeño resumen del proyecto, donde se incorpora información de los autores, ruta de trabajo realizada, soporte fotográfico y evidencias fílmicas de las misiones realizadas. El módulo de adquisición de datos, ver figura 7.17 es responsable de realizar la 


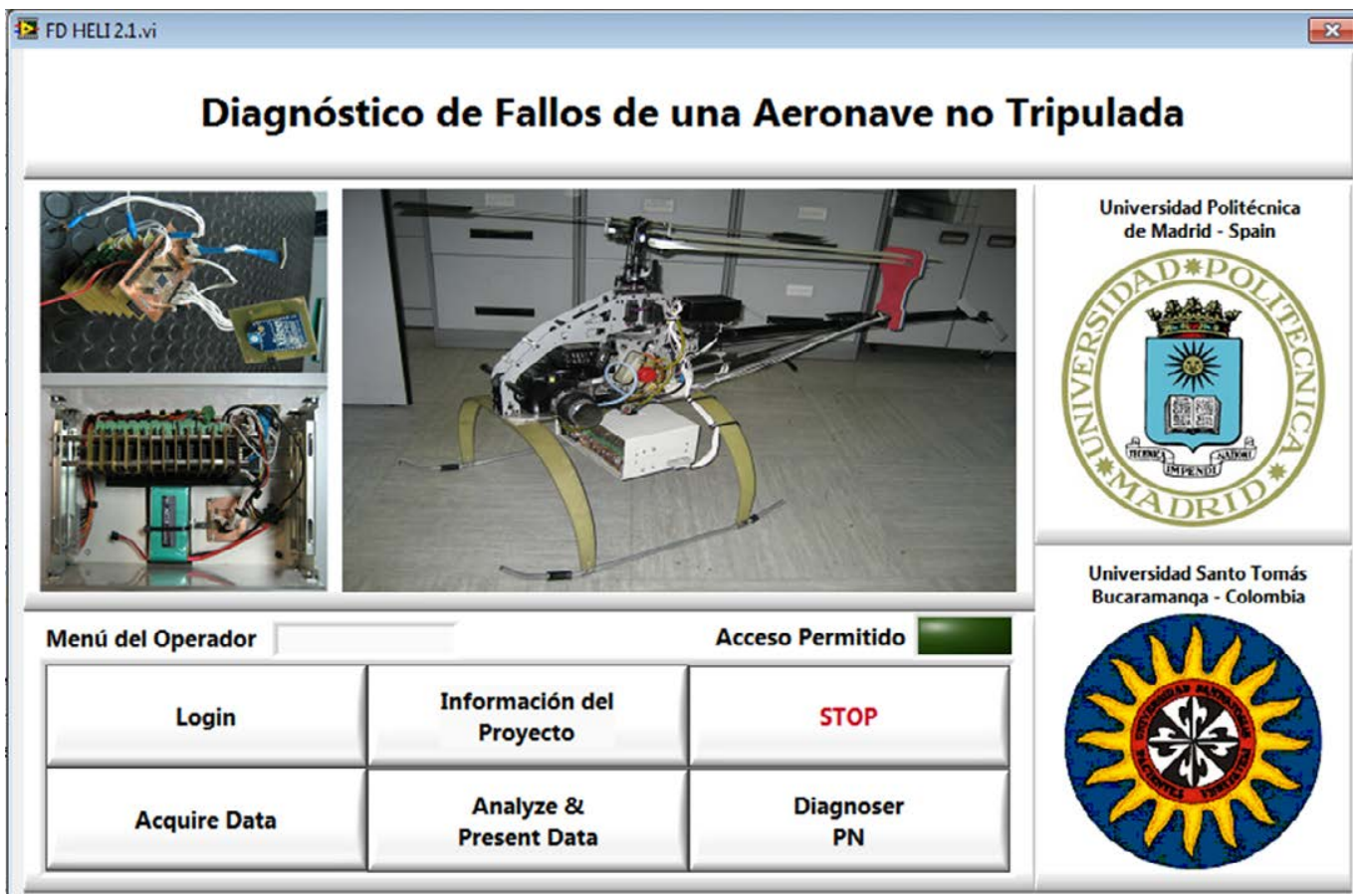

Figura 7.16: Interfaz Gráfica LabVIEW

adquisición de datos en tiempo real. Los datos son recogidos y visualizados de una manera on-line y almacenados en un archivo para su posterior uso. En esta ventana también se observan salidas digitales que representan los fallos.

El modulo de análisis y presentación de datos permite realizar análisis offline de datos de las diferentes misiones, ver figura 7.18. Finalmente, se ha implementado un modulo para que se ejecute la Red de Petri diagnosticadora, el cual es capaz de manejar datos en tiempo real y datos almacenados de misiones pasadas. La RdPD registra la frecuencia con el cual cualquier componente en la aeronave cae en alarma o fallo, ver figura 7.19.

\subsection{Aplicación de la Herramienta de FD al Helicóptero}

La UAV elegida es un helicóptero, que ha sido explicado en detalle en el apartado anterior. Es una aeronave que ha sido utilizada en el laboratorio del grupo de investigación de Robótica y Cibernética de la Universidad Politécnica de Madrid; ha hecho parte de múltiples investigaciones sobre técnicas de control; su desempeño y buen funcionamiento ha sido fundamental en el grupo. Una falla del helicóptero, como ha sucedido en varias ocasiones, puede ocasionar que las diferentes pruebas de las investigaciones se vean afectadas y lo 


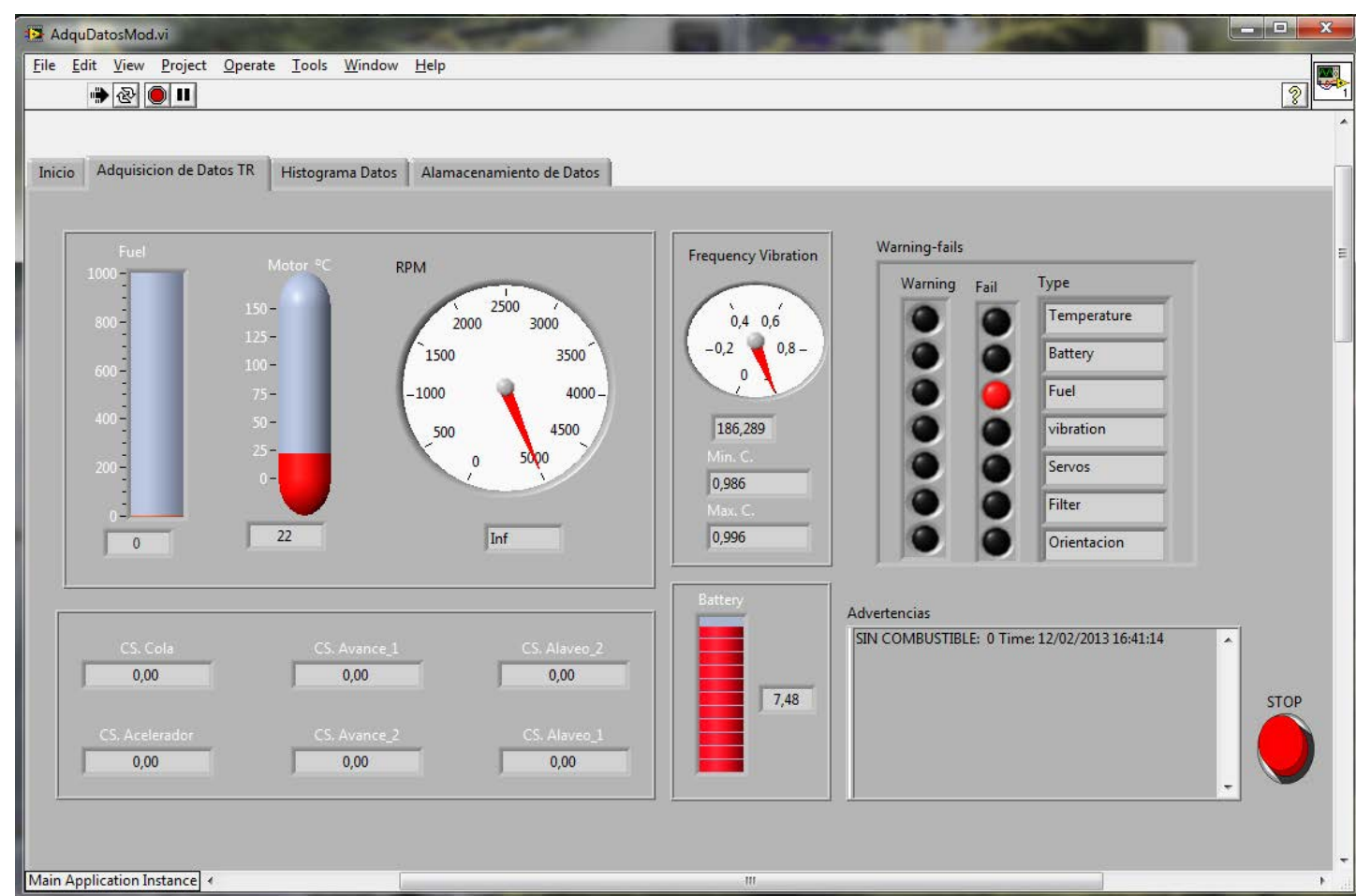

Figura 7.17: Modulo DAQ LabVIEW

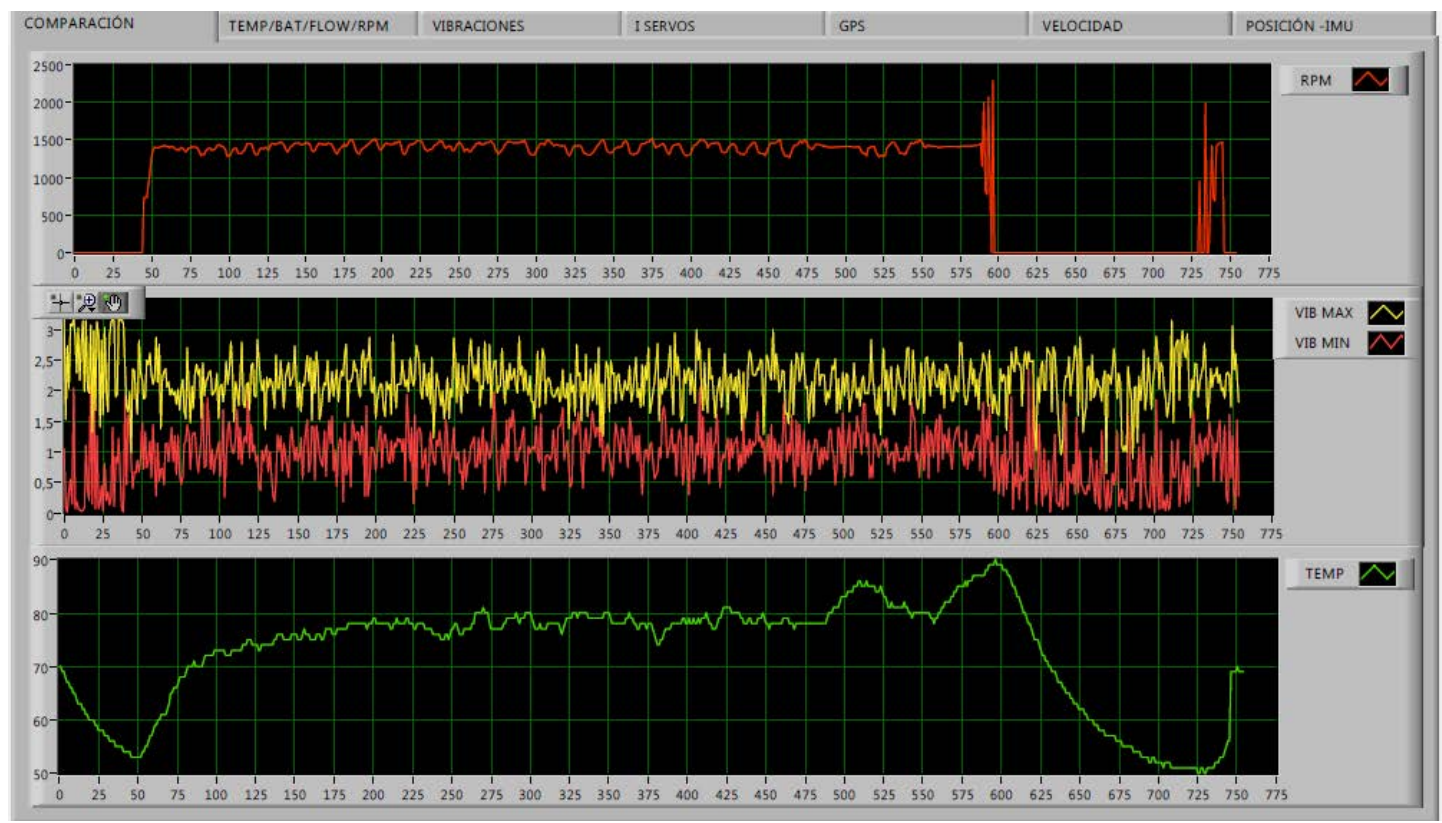

Figura 7.18: Modulo Análisis y Presentación de Datos 


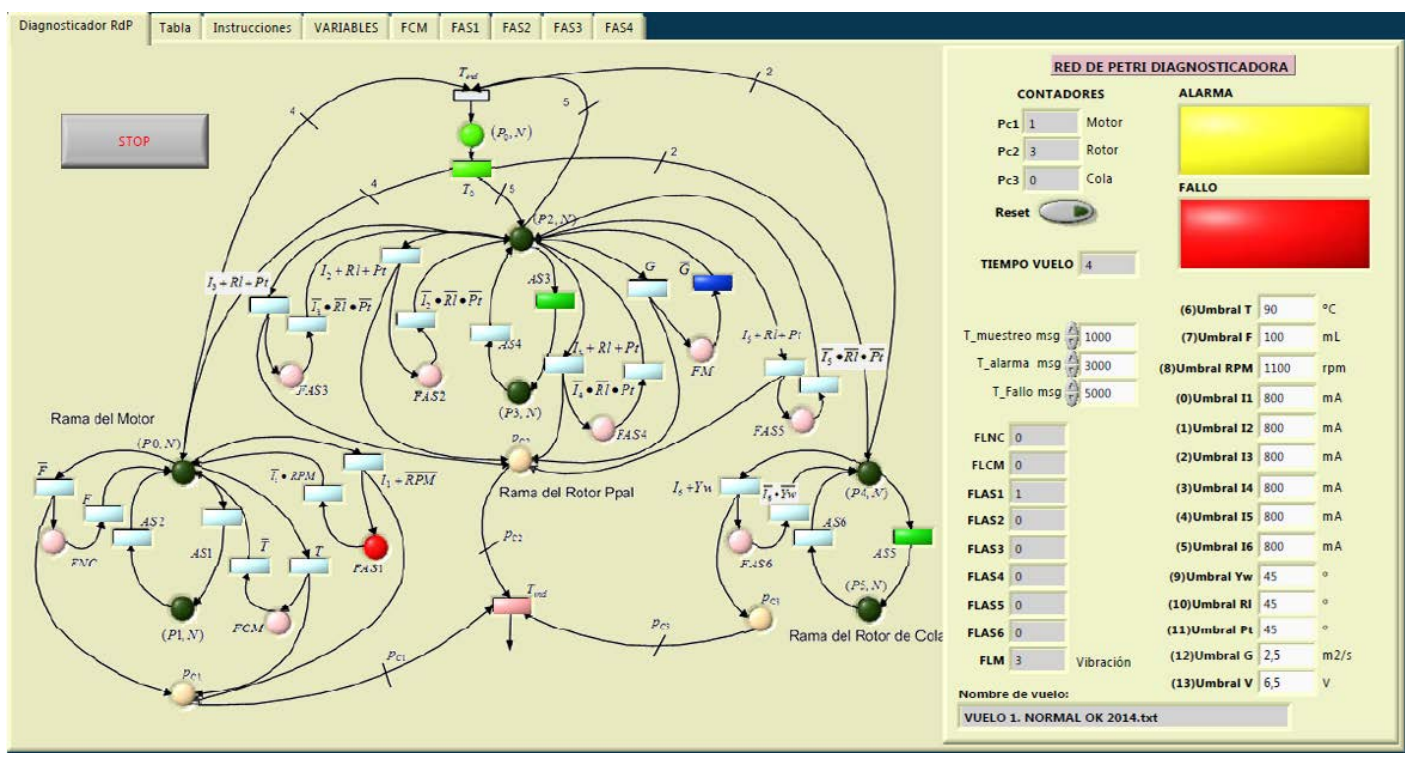

Figura 7.19: Modulo Red de Petri Diagnosticadora

que es peor, no se conozcan detalles de las causas por las que la aeronave no funciono correctamente.

A continuación vamos a proceder a aplicar la herramienta de FD al HC, es un proceso sistemático y que busca determinar las condiciones de la aeronave en una misión, el diagnosticador evalúa el comportamiento y será el piloto en la estación en tierra, quien determina si debe abortar la misión. Previo a la aplicación del algoritmo, debemos realizar los siguientes supuestos:

- El Helicóptero debe ser encendido manualmente.

- Se asume que ningún fallo puede ocurrir en los controladores, no esta contemplado en la aplicación del proceso.

- No se tienen en cuenta los fallos que pueden ocurrir en el sistema de comunicaciones.

Basado en la importancia de los tres sistemas fundamentales que conforman el helicóptero, es a ellos a quienes se enfoca la clasificación de los subsistemas, seguido se hace el modelo de RdP de los dispositivos que los componen, en cada dispositivo se tiene en cuenta su estado normal y de fallo. Posteriormente se construye el modelo general de RdP de cada subsistema, que se convertirá en un modelo refinado libre de transiciones no observables $T_{u o}$. Por último, el diagnosticador integra los modelos refinados y a través de las rama asignada 


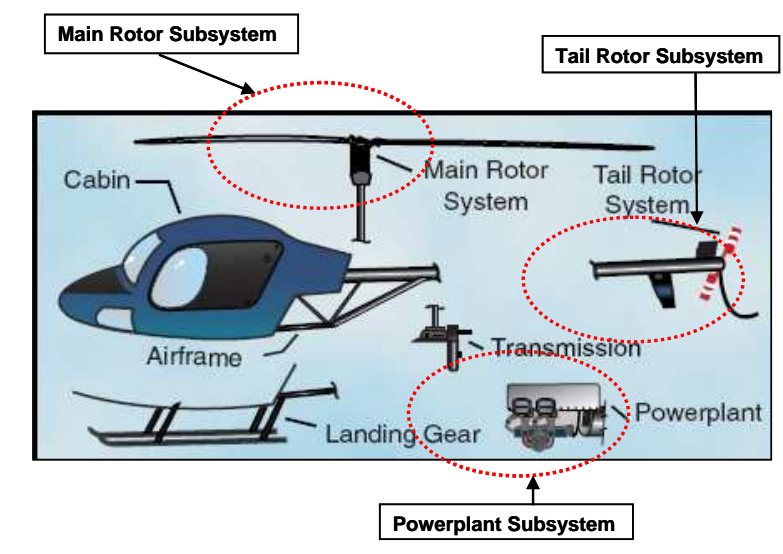

Figura 7.20: Clasificación de los Subsistemas del Helicóptero.

a cada fallo, se realiza un diagnóstico de los fallos individualmente, detectando fallos simultáneamente y teniendo en cuenta los fallos de otros elementos en diagnóstico.

\section{Paso 1. Clasificación en Subsistemas.}

El helicóptero $(H)$ es clasificado en subsistemas fundamentales como muestra la figura 7.20 , el subsistema del motor $\left(H_{1}\right)$, el subsistema del rotor principal $\left(\mathrm{H}_{2}\right)$ y el subsistema del rotor de cola $\left(H_{3}\right)$.

- Subsistema del Motor: Encargado de la generación del movimiento que va a ser transmitido al rotor principal (plato). Compuesto por el motor y el tanque de combustible.

- Subsistema del Rotor Principal: Recibe el movimiento del motor y lo transmite a las palas del HC a través de cuatro servos.

- Subsistema del Rotor de Cola: Recibe el movimiento del motor y en sincronía con el rotor del plato, lo transmite a las palas traseras a través de un servo.

Para el funcionamiento de la electrónica embarcada en la aeronave, se requiere de una fuente de alimentación constante y con un valor de voltaje mínimo, esta variable no esta incluida como fallo en el proceso, pero es medida y será adicionada en el diagnosticador como condición de funcionamiento. 
CAPÍTULO 7. IMPLEMENTACIÓN Y PRUEBAS DE CAMPO DEL DIAGNOSTICADOR EN EL HC NO TRIPULADO.

Paso 2. Construir el modelo de RdP de los componentes de cada subsistema.

A continuación se procede a la elaboración de los modelos de RdP de cada uno de los dispositivos que componen los subsistemas. Se seguirán las pautas definidas en el proceso de la sección 6.2. El sistema cuenta con tres subsistemas, $M=3$.

SUBSISTEMA DEL MOTOR $H_{1}$ : El subsistema tiene dos componentes para modelar: El controlador $H_{1}^{1}$ y el servo del acelerador $H_{1}^{2}$. Gracias a la inyección de combustible en la combustión, las revoluciones del motor varían y por consiguiente la velocidad de las palas de los rotores. Basado en la experiencia tomada de la sección 7.0.2, se evalúan las siguientes variables en el motor:

- Flujo de combustible $(F)$ : Con esta variable, el controlador evalúa la cantidad de combustible consumido en vuelo y lo compara con el parámetro de referencia del tanque en su máximo nivel, con ello puede establecer el tiempo restante de misión por almacenamiento de combustible. El tanque almacena $500 \mathrm{ml}$ y no debe de tener en un nivel inferior a $100 \mathrm{ml}$.

- Temperatura del motor $(T)$ : Una de las formas de evidenciar problemas en el motor, es a través del incremento de la temperatura del motor, las causas pueden ser por: desgaste del sistema interno del motor, problemas en la sincronización de la mezcla de combustible, fallos mecánicos en la transmisión del movimiento con el plato, entre otros. Se pudo establecer que la temperatura de funcionamiento normal no debe exceder a $90^{\circ}$ grados celsius.

- Velocidad del motor $(R P M)$ : El motor necesita tener unas $R P M$ mínimas que garanticen el giro de las palas y unas $R P M$ máximas que no sobreesfuercen la aeronave, se hace necesario medirlas y mantener en un nivel mínimo en vuelo normal, éstas deben estar en un rango entre 1200 y 1500 RPMs. La reducción o variación de las $R P M$ evidencian posibles problemas en el combustible, fallo del servo de inyección de combustible, fallo mecánico en la transmisión del movimiento, entre otros.

- Corriente consumida por el servo del acelerador $\left(I_{1}\right)$ : Existe la posibilidad de que el servomotor encargado de regular la inyección de combustible en la mezcla del motor se estropee, por esta razón se evalúa la 
corriente del servo y con ello se establece que no hay problemas en su composición mecánica interna y externa, que conlleva a un atascamiento. El comportamiento de la corriente del servo esta en un rango de 100 a $600 \mathrm{~mA}$ en funcionamiento normal y si supera un valor de $800 \mathrm{~mA}$ en fallo, evidenciamos que el servo está en posible fallo.

Los fallos a diagnosticar en la aeronave son:

- Fallo de Calentamiento del Motor ( $F C M)$ : El umbral de temperatura normal del motor es de $90^{\circ}$ celsius, es decir que si la aeronave pasa por encima de $90^{\circ} \mathrm{C}$, es una condición de fallo.

- Fallo del nivel de combustible en el tanque ( $F N C$ ): El nivel máximo de combustible en el tanque es de $500 \mathrm{~mL}$, para garantizar la misión, se debe mantener como nivel mínimo $100 \mathrm{ml}$ en el tanque y proceder a abortar la misión.

- Fallo de atascamiento del Servo de combustible ( $F A S 1)$ : EL servo siempre tendrá una posición que garantiza el paso de combustible hacia el motor, en esta posición (energizado, pero sin actuación) el servo tiene un consumo de corriente, que varía en un rango de 100 a $600 \mathrm{~mA}$, cuando recibe una orden del controlador el consumo de corriente se incrementa debido al accionamiento mecánico. por lo anterior, una condición de fallo, es cuando hay un consumo superior a $800 \mathrm{~mA}$ de corriente, debido a que hay un atascamiento del servo o sencillamente el acople mecánico se ha roto.

En la figura 7.21 se muestra el modelo de RdP del controlador y del servo. En ellos se evidencia la integración en cada modelo de RdP del funcionamiento normal y de los tres fallos nombrados anteriormente. También, se puede observar que las transiciones de fallo son no-observables $\left(T_{u o}\right)$ y junto con los lugares de fallo son representados por barras y círculos sombreados. El modelo de RdP del controlador es la abstracción de su funcionamiento, para efectos de un mejor entendimiento su funcionamiento normal se representa como un ciclo cerrado. La función del controlador es abrir o cerrar la inyección de combustible, dependiendo de las necesidades del piloto (automático o manual). El controlador está en su lugar de inicio $C 1$, recibe la orden de una posición requerida, representada por la la transición $A S 1$, indica al servo actuar, de este modo 

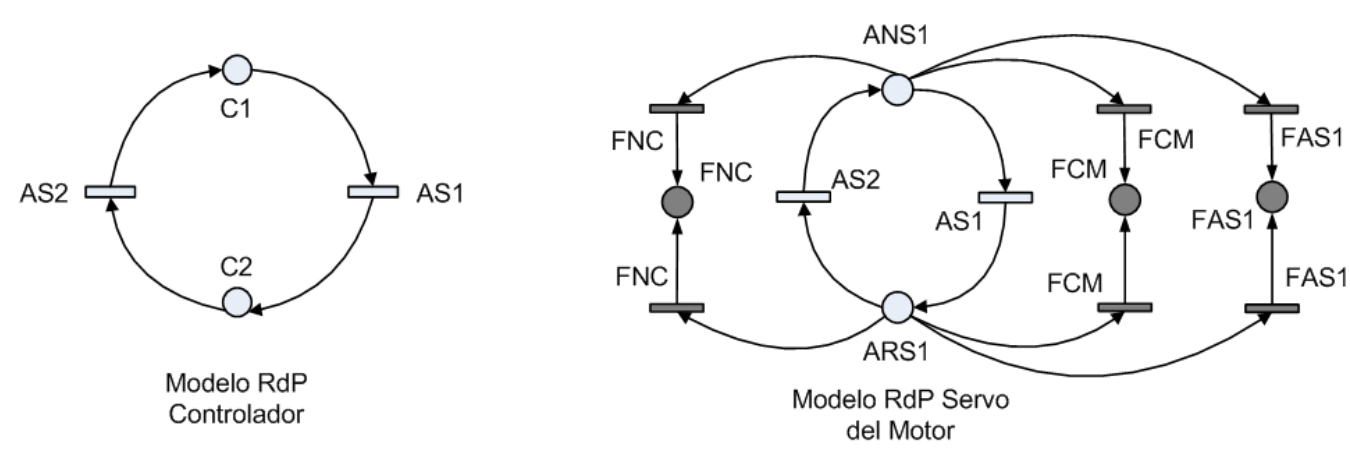

Figura 7.21: Modelo de RdP de componentes del Subsistema del Motor.

pasa a una posición diferente, lugar $C 2$, igual estando en el lugar $C 2$, recibe una nueva ubicación, la transición $A S 2$ y se desplaza a otra posición, que la representamos como el lugar $C 1$. En el modelo RdP del servo de inyección de combustible, funciona del mismo modo que el controlador. Estando en un lugar de inicio y actuación normal ( $A N S 1$ ) recibe la orden del controlador, transición $A S 1$, pasa a un lugar de actuación requerida $(A R S 1)$, posteriormente, el controlador emite la transición $A S 2$ y el servo pasa a su posición normal $A N S 1$. Dado que la prioridad del motor es que nunca se vea afectado su funcionamiento en vuelo, se representan en el modelo de RdP los fallos de calentamiento del motor $F C M$, fallo de nivel de combustible $F N C$ y fallo de atascamiento del servo de combustible (servo 1) FAS1. Como se dijo anteriormente, los fallos ocurren estando en cualquier estado del servo y del sistema.

Subsistema del Rotor Principal (Plato) $\mathrm{H}_{2}$ : Hay dos componentes para modelar en el subsistema del plato, que son: servos del plato y controlador. Los cuatro servos encargados de hacer girar el plato del HC, y el controlador es quien envía indicaciones a los cuatro servos que realizan el movimiento del plato. Las variables monitoreadas son:

- Corriente consumida por los servos del plato $\left(I_{2}, I_{3}, I_{4}, I_{5}\right)$ : Basado en la experiencia en el manejo de las aeronaves, es posible que aunque se atasque un servo, se pueda mantener control de la aeronave para abortar la misión, por lo anterior justifica medir la corriente del servo en todo momento.

- Angulo Roll y Pitch $(R l, P t)$ : Un cambio fuerte de posición en la aeronave indica que existen problemas en el plato, medir los ángulos de actitud nos 
CAPÍTULO 7. IMPLEMENTACIÓN Y PRUEBAS DE CAMPO DEL DIAGNOSTICADOR EN EL HC NO TRIPULADO.

informan un buen funcionamiento de la misión. Un angulo inferior a $45^{\circ}$ es síntoma de normalidad en el vuelo.

- Vibraciones de cola $(G)$ : Vibraciones en cualquier parte de la aeronave indican un mal funcionamiento del sistema mecánico, conocerlas al amplitudes de estos movimientos nos ayuda a evitar que los componentes mecánicos pierdan su sincronía y la aeronave se precipite a tierra. La Vibraciones se miden en unidades $\mathrm{G}$ que están representadas como fracciones de la aceleración debido a la gravedad, la amplitud máxima permitida es de $2.5 \mathrm{G}$.

Los fallos considerados en el diagnosticador son:

- Fallo de Atascamiento de los Servos del Rotor Principal ( $F A S 2, F A S 3$, $F A S 4, F A S 5)$ : El fallo de atascamiento de los servos es evaluado a través de la corriente consumida y por los ángulos Roll y Pitch de la aeronave. En la posición de sostenimiento el servo del plato normalmente consume una corriente que varía en un rango de 100 a $600 \mathrm{~mA}$, en el cambio de posición del servo, la corriente se incrementa. Cuando el valor de corriente del servo es superior a $800 \mathrm{~mA}$, es debido a un atascamiento de la estructura mecánica del servo. En cuanto la posición de la aeronave, los ángulos Roll

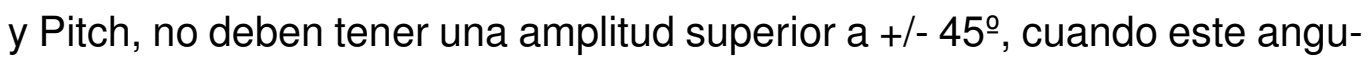
lo se sobrepasa, puede ser debido a que uno o mas servos no funcionan correctamente y la aeronave se puede precipitar a tierra.

- Fallo Mecánico del Chasis $(F M)$ : Un fallo mecánico es el resultado del desajuste de algún componente mecánico de la aeronave, que puede ser generado por diferentes motivos, entre los mas importantes están: Perdidas de tornillos, rotura de soldaduras, descompesación de palas del plato o cola, entre otros. La máxima amplitud permitida es de $2.5 G$, si este valor se ve superado estando la aeronave en altura, nos puede llevar a problemas catastróficos.

En la figura 7.22 se muestra el modelo de RdP del controlador y el servo. La función del controlador es establecer la orden del paso de los diferentes servos a las posiciones requeridas. Estando en una posición actual $(C 3)$ recibe la orden, transición $A S 3$, de desplazarse a una posición requerida $(C 4)$, posteriormente recibe de nuevo la orden de desplazarse a una nueva ubicación, denominada 

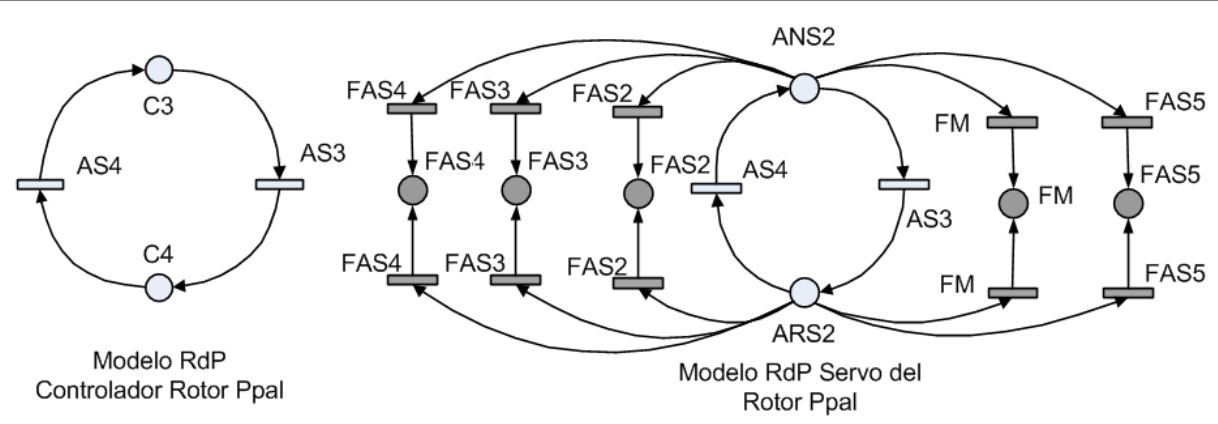

Figura 7.22: Modelo de RdP de los Componentes del Subsistema del Rotor Principal.

$C 3$, con la orden representada por la transición $A S 4$. En esta misma figura se observa el modelo de RdP del servo, Aunque existen cuatro servos en el plato, por efectos de simplicidad son representados por un solo modelo. En su modelo se observa que el servo está en una posición normal ( $A N S 2)$, recibe la orden del controlador por medio de la transición $A S 3$, y pasa a una posición requerida $(A R S 2)$, vuelve a su posición siguiente, representada como $A N S 2$, a través de una nueva orden del controlador, transición $A S 4$. En este subsistema se diagnostican los fallos de atascamiento de los servos ( $F A S 2, F A S 3, F A S 4, F A S 5$ ), que son evaluados por medio de la corriente medida en cada servo y el fallo mecánico del chasis $(F M)$. El modelo construido es idéntico para cada uno de los cuatro servos que componen el plato.

Subsistema del Rotor de Cola $H_{3}$ : Dos componentes han sido considerados en el modelo: servo de cola y controlador. El servo es el responsable de hacer girar la cola del HC, y el controlador (giróscopo) quien envía indicaciones al servo para realizar el movimiento de las palas de cola, que cumple la función antitorque (Angulo Yaw). Es imposible que un $\mathrm{HC}$ funcione sin el rotor de cola y el atascamiento de su servo hace que la aeronave gire sobre su mismo eje. Se busca medir la siguiente variable en el rotor de cola del HC:

Las variables monitoreadas son:

- Corriente consumida por el servo de cola $\left(I_{6}\right)$ : Su comportamiento es el mismo presentado en los servos del rotor principal.

- Ángulo Yaw $(Y w)$ : Existen situaciones donde la aeronave puede realizar autogiros, pero cuando se prolongan puede existir una avería del servo de cola. 


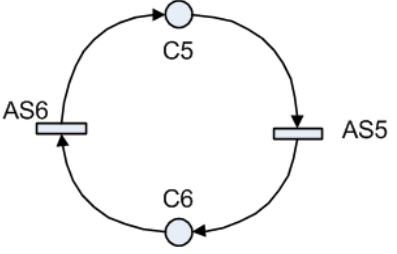

Modelo RdP Controlador Rotor de Cola

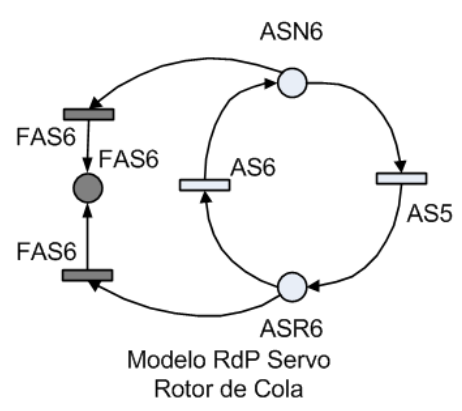

Figura 7.23: Modelo de RdP de los Componentes del Subsistema del Rotor de Cola.

El fallo a diagnosticar es un fallo de atascamiento de servo en la cola FSS6. EI rotor de cola está compuesto de un único servo y depende directamente de las señales emitidas por el giróscopo.

- Fallo de Atascamiento del Servo de Cola $F A S 6$ : El fallo de atascamiento del servo es evaluado a través de la corriente consumida y el angulo pitch de la posición de la aeronave. En la posición de sostenimiento el servo del plato normalmente consume una corriente que oscila entre 100 y $600 \mathrm{~mA}$, en el cambio de posición del servo, la corriente se incrementa. Cuando el valor de corriente del servo es superior a $800 \mathrm{~mA}$, se debe a un atascamiento de la estructura mecánica del servo. Por otro lado, el angulo yaw no puede superar $+/-45^{\circ}$, por un tiempo prolongado, si este valor es superado, la aeronave puede girar sobre su propio eje y su causa sea el malfuncionamiento del servo de cola.

En la figura 7.23 se presenta el modelo abstracto del controlador y el servo. Se puede observar el modelo de RdP del controlador, situado en una posición normal o actual $(C 5)$, recibe la transición de referencia $A S 5$ para pasar a un nuevo lugar $(C 6)$, el controlador ubicado en el lugar requerido $(C 6)$ repite continuamente la actuación, es decir emite una nueva transición de actuación $A S 6$ y pasa al nuevo lugar de referencia $(C 5)$. En el modelo de RdP del servo del rotor de cola, está en una posición de actuación normal o actual $(A S N 3)$ y a la orden del controlador, transición ( $A S 5$ ) pasa a una actuación requerida ( $A S R 3)$, el proceso es continuo al recibir la transición ( $A S 6)$ para regresar a su nuevo lugar. El fallo a diagnosticar en este subsistema, es el fallo de atascamiento del servo del rotor de cola ( $F A S 6)$. 


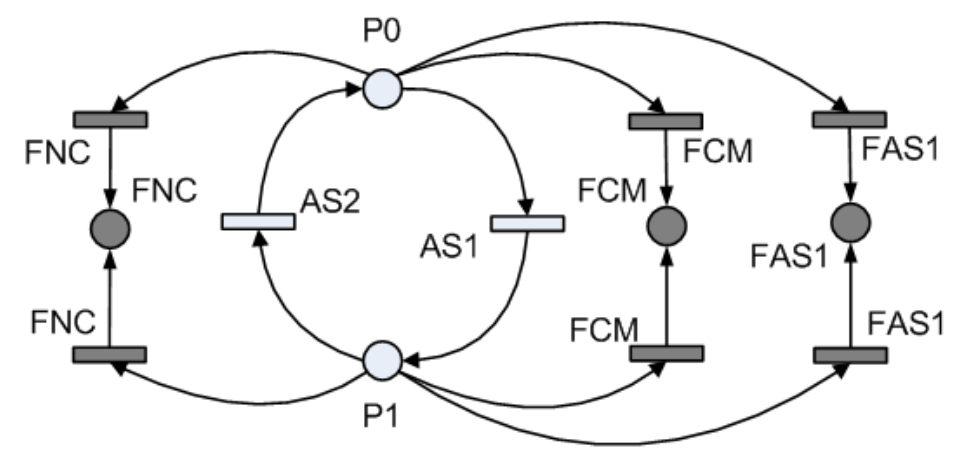

Figura 7.24: Modelo General de RdP del Subsistema del Motor.

\section{Paso 3. Operación de Integración.}

Consiste en unir en un solo modelo general a los modelos de RdP de cada uno de los componentes de su correspondiente subsistema $j$, con el objetivo de ver en un solo modelo el funcionamiento normal y de fallo de cada subsistema. En el modelo de integración se mantienen los lugares y transiciones de fallo existentes en los modelos individuales, y se hace la unión de los lugares normales. Para la construcción del modelo general, se usa como referencia el proceso descrito en la sección 6.2.1.

\section{- Subsistema del Motor.}

El conjunto de fallos del motor es $F_{1}=\{F C M, F N C, F A S 1\}$. El lugar de inicio $P 0=\{C 1, A N S 1\}$ es la unión de los lugares de inicio del controlador $C_{1}$ y el lugar de inicio de la actuación normal del servo de combustible $A N S 1$. Este lugar de inicio hereda la marca inicial, tal como en el modelo individual. Construimos las rama de integración de funcionamiento normal de los modelos individuales, mantiene la sincronía de los modelos individuales, las transiciones dependen del controlador y marcan la evolución de la RdP. Se adicionan los lugares de fallo por cada fallo del conjunto $F_{1}$. Desde cada lugar de la rama normal se puede disparar las transiciones de fallo, por lo tanto, cada lugar de fallo es unido a cada lugar normal por medio de su respectiva transición de fallo, esta transición es no-observable. Finalmente, el otro lugar de la RdP general del motor $P 1$ esta compuesto por $C 2$ y $A R S 1$. El modelo general se puede ver en la figura 7.24.

\section{- Modelo RdP General del Subsistema del Rotor Principal.}




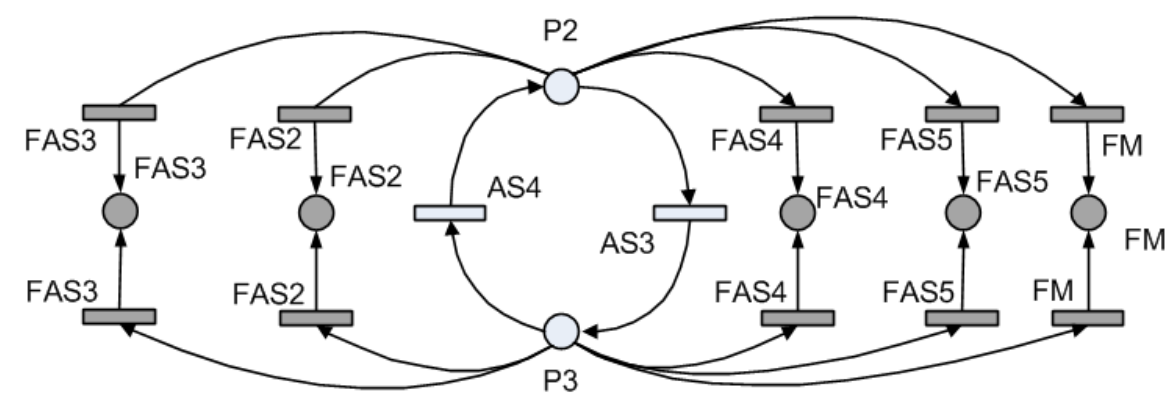

Figura 7.25: Modelo General de RdP del Subsistema del Rotor Principal

El conjunto de fallos del rotor principal es $F_{2}=\{F A S 2, F A S 3, F A S 4, F A S 5$, $F M\}$. El lugar de inicio $P 2=\{C 3, A N S 2\}$ es la unión de los lugares de inicio del controlador $C 3$ y los lugares de inicio de la actuación normal de los servos del rotor $A N S 2, A N S 3, A N S 4, A N S 5$. Construimos las rama de integración de funcionamiento normal de los modelos individuales. Se adicionan los lugares de fallo del conjunto $F_{2}$. Agregamos las transiciones de fallo. El otro lugar de la RdP general del motor P3 esta compuesto por $C 4, A R S 2, A R S 3, A R S 4, A R S 5$. El modelo general se puede observar en la figura 7.25 . .

\section{- Modelo de RdP General del Subsistema del Rotor de Cola.}

El conjunto de fallos del rotor de cola es $F_{3}=\{F A S 6\}$. El lugar de inicio $P 4=\{C 5, A N S 6\}$ es la unión de los lugares de inicio del controlador $C 5$ y los lugares de inicio de la actuación normal de los servos del rotor $A N S 6$. Construimos las rama de integración de funcionamiento normal de los modelos individuales. Se adicionan los lugares de fallo del conjunto $F_{3}$. Agregamos las transiciones de fallo. El otro lugar de la RdP general del motor $P 5$ esta compuesto por $C 6, A R S 6$. El modelo general se puede ver en la figura 7.26.

\section{Paso 4. Refinar el Modelo General.}

El posterior paso en el proceso de FD, se hace necesario refinar el modelo de RdP general, debido a que se debe sustituir las transiciones de fallo (noobservables $T_{u o}$ ) por transiciones observables $T_{o}$; este proceso se realiza basado en las variables medidas $T_{S}$ (sensores) con que cuenta el sistema. Para ello se construye la tabla de integración de sensores, que es la productoria de las 


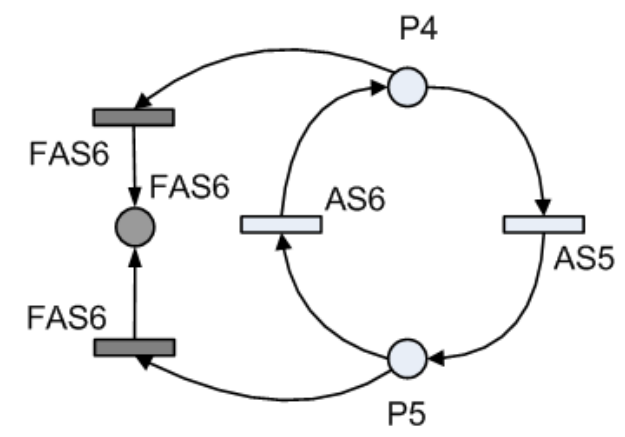

Figura 7.26: Modelo General de RdP del Subsistema del Rotor de Cola.

salidas de las lecturas sensoriales, comparadas con las lecturas esperadas del lugar. Cuando el subsistema está en cualquier lugar del funcionamiento normal, los sensores pueden entregar medidas diferentes a las esperadas, indicando la presencia del fallo. Esta lectura sensorial reemplaza a la $T_{u o}$ de fallo y el modelo de RdP es así como queda compuesto solo de $T_{o}$ y por lo tanto, queda refinado. La tabla de integración de sensores se construye individualmente para cada subsistema.

- Paso 4.1. Identificar los Sensores Ns para cada Subsistema, acorde a los sensores requeridos para monitorear las variables deseadas. Como previamente se mencionó, el conjunto de sensores del motor está compuesto del sensor de flujo de combustible $F$, Un sensor de temperatura $T$, un sensor de velocidad (encoder) $R P M$ y un sensor de corriente del servo de combustible $I_{1}$. El conjunto $N s$ para el subsistema del motor es por lo tanto definido como sigue: $N s 1=F, T, R P M, I_{1}$. Siguiendo el mismo procedimiento, el conjunto de sensores del rotor principal es $N s 2=I_{2}, I_{3}, I_{4}, I_{5}, R l, P t, G$, el cual es compuesto por cuatro sensores de corriente para los servos del plato $(I 2, I 3, I 4, I 5)$, un sensor inercial que estima los ángulos roll y Pitch $(R l, P t)$ y un acelerómetro para medir las vibraciones en el chasis $G$. Finalmente, el conjunto de sensores del rotor de cola esta compuesto por dos sensores, un sensor de corriente del servo de cola $I_{6}$ y un compás magnético para estimar el cabeceo o ángulo yaw $Y w$. Subsecuentemente, el conjunto es $N s 3=I_{6}, Y w$.

- Paso 4.2. Construir el Conjunto Discreto de Salidas de SensoRES. Cada sensor del sistema entregará una medida discreta, que define 
su estado; con éstas medidas se construye el conjunto $Y$ de salidas de los sensores y nos permite establecer con cuales lecturas se cuenta en la evaluación de cada subsistema. Se construye cada conjunto individualmente en cada subsistema. Acorde a definiciones previas: el subsistema del motor tiene $\left|Y_{1}\right|=2^{N} s=2^{4}=16$ combinaciones. Acorde a esta formulación, el subsistema del rotor principal tiene $\left|Y_{2}\right|=16$ combinaciones (note que los cuatro sensores de corriente del plato son representados solo por un solo valor: Is). El subsistema del rotor de cola tiene por lo tanto $\left|Y_{3}\right|=4$. Cada lectura es medida de una forma discreta.

- Subsistema del Motor: Compuesto por los sensores de flujo $F$, temperatura $T$, revoluciones por minuto $R P M$ y corriente del servo de combustible $I_{1}$. $\left|Y_{1}\right|=2^{N s}=2^{4}=16$, combinaciones.

- Subsistema del Rotor Principal: Compuesto por los sensores de corriente de cada servo del rotor principal, $I_{2}, I_{3}, I_{4}, I_{5}$ las medidas de los ángulos $R l, P t$, y la medida de las vibraciones $G$. Para efectos de simplicidad se representa una sola corriente de servo denominada Corriente de servo Is. $\left|Y_{2}\right|=2^{4}=16$.

- Subsistema del Rotor de Cola: Compuesto por el sensor de corriente del rotor de cola y la medida del angulo Yaw $Y w, Y_{6} .\left|Y_{3}\right|=2^{2}=4$.

Las combinaciones de salidas sensoriales de los tres subsistemas se pueden observar en la tabla 7.2. Las salidas sensoriales se convierten en las entradas de la tabla de integración se sensores.

- Paso 4.3. Definir las Salidas de la Tabla de InTegración. Para cada lugar del modelo general de cada subsistema, se agrega una salida en la tabla de integración de sensores, esta salida contiene la representación de las lecturas del subsistema en un estado de funcionamiento normal.

- Subsistema del motor: dos salidas han sido adicionadas para el subsistema del motor, es decir $P 0$ y $P 1$. Bajo condiciones de funcionamiento normal, la lectura de combustible debe ser $F$ (es decir, el combustible en el tanque está por encima del umbral mínimo de $100 \mathrm{ml}$ ), la lectura de la temperatura debe ser $\bar{T}$ (es decir, la temperatura del motor está por debajo del umbral, $90^{\circ} \mathrm{C}$ ), el valor para la velocidad 
CAPÍTULO 7. IMPLEMENTACIÓN Y PRUEBAS DE CAMPO DEL DIAGNOSTICADOR EN EL HC NO TRIPULADO.

Tabla 7.2: Conjunto Discreto de Salidas de Sensores de los Subsistemas del $\mathrm{HC}$

\begin{tabular}{|c|c|c|c|c|c|c|c|c|c|c|}
\hline & \multicolumn{4}{|c|}{ Lecturas Motor } & \multicolumn{4}{|c|}{ Lect Rotor Ppal } & \multicolumn{2}{|c|}{ Lect Rotor Cola } \\
\hline Combinaciones & $F$ & $T$ & $R P M$ & $I_{1}$ & $I_{S}$ & $R l$ & $P t$ & $G$ & $I_{6}$ & $P t$ \\
\hline 1 & $\overline{\bar{F}}$ & $\overline{\bar{T}}$ & $\overline{R P M}$ & $\overline{\overline{I_{1}}}$ & $\overline{\overline{I_{S}}}$ & $\overline{R l}$ & $P t$ & $\overline{\bar{G}}$ & $\overline{\overline{I_{6}}}$ & $\overline{Y w}$ \\
\hline 2 & $\bar{F}$ & $\bar{T}$ & $\overline{R P M}$ & $I_{1}$ & $\overline{I_{S}}$ & $\overline{R l}$ & $P t$ & $G$ & $\overline{I_{6}}$ & $Y w$ \\
\hline 3 & $\bar{F}$ & $\bar{T}$ & $R P M$ & $\overline{I_{1}}$ & $\overline{I_{S}}$ & $\overline{R l}$ & $\overline{P t}$ & $\bar{G}$ & $I_{6}$ & $\overline{Y w}$ \\
\hline 4 & $\bar{F}$ & $\bar{T}$ & $R P M$ & $I_{1}$ & $\overline{I_{S}}$ & $\overline{R l}$ & $\overline{P t}$ & $G$ & $I_{6}$ & $Y w$ \\
\hline 5 & $\bar{F}$ & $T$ & $\overline{R P M}$ & $\overline{I_{1}}$ & $\overline{I_{S}}$ & $R l$ & $P t$ & $\bar{G}$ & & \\
\hline 6 & $\bar{F}$ & $T$ & $\overline{R P M}$ & $I_{1}$ & $\overline{\bar{I}_{S}}$ & $R l$ & $P t$ & $G$ & & \\
\hline 7 & $\bar{F}$ & $T$ & $\overline{R P M}$ & $\overline{\overline{I_{1}}}$ & $\overline{I_{S}}$ & $R l$ & $\overline{P t}$ & $\bar{G}$ & & \\
\hline 8 & $\bar{F}$ & $T$ & $R P M$ & $I_{1}$ & $\overline{I_{S}}$ & $R l$ & $\overline{P t}$ & $G$ & & \\
\hline 9 & $F$ & $\bar{T}$ & $\overline{R P M}$ & $\overline{\overline{I_{1}}}$ & $I_{S}$ & $\overline{R l}$ & $P t$ & $\bar{G}$ & & \\
\hline 10 & $F$ & $\bar{T}$ & $\overline{R P M}$ & $I_{1}$ & $I_{S}$ & $\overline{R l}$ & $P t$ & $G$ & & \\
\hline 11 & $F$ & $\bar{T}$ & $R P M$ & $\overline{I_{1}}$ & $I_{S}$ & $\overline{R l}$ & $\overline{\overline{P t}}$ & $\bar{G}$ & & \\
\hline 12 & $F$ & $\overline{\bar{T}}$ & $R P M$ & $I_{1}$ & $I_{S}$ & $\overline{\overline{R l}}$ & $\overline{\overline{P t}}$ & $G$ & & \\
\hline 13 & $F$ & $\bar{T}$ & $\overline{R P M}$ & $\overline{I_{1}}$ & $I_{S}$ & $R l$ & $P t$ & $\bar{G}$ & & \\
\hline 14 & $F$ & $T$ & $\overline{R P M}$ & $I_{1}$ & $I_{S}$ & $R l$ & $P t$ & $G$ & & \\
\hline 15 & $F$ & $T$ & $R P M$ & $\overline{I_{1}}$ & $I_{S}$ & $R l$ & $\overline{P t}$ & $\bar{G}$ & & \\
\hline 16 & $F$ & $T$ & $R P M$ & $I_{1}$ & $I_{S}$ & $R l$ & $\overline{P t}$ & $G$ & & \\
\hline
\end{tabular}

del rotor debe ser $R P M$ (es decir, las revoluciones del motor están por encima del umbral de $1200 \mathrm{rpm}$ ) y las lecturas de corriente de los servos debe ser $\bar{I}$ (es decir, la corriente de los servos está en el rango que representa el funcionamiento normal y no bloqueo, 100 to $600 \mathrm{~mA})$. Ver tabla 7.3.

- Subsistema del rotor principal: dos salidas han sido incluidas en la tabla para $P 2$ y $P 3$. La lectura del sensor de corriente $\overline{I_{s}}$ debe estar en el mismo rango que el servo del motor. Las lecturas de los ángulos de posición (roll y pitch) deben ser $\overline{R l}, \overline{P t}$ (es decir, un ángulo inferior a $45^{\circ}$ ) y las vibraciones deben ser $\bar{G}$ (es decir, inferior a $2.5 \mathrm{~g}$ ), ver tabla7.4

- Subsistema del rotor de cola: dos salidas han sido incorporadas a la tabla para $P 4$ y $P 5$. Las lecturas de la corriente de servo deben ser $\overline{I_{6}}$ y la lectura del ángulo yaw debe $\operatorname{ser} \overline{Y w}$. Ver tabla 7.5

- Paso 4.4. Construcción de la Tabla de Integración de SensoRES. Las lecturas de los sensores $Y$ tienen que verificarse de manera 
CAPÍTULO 7. IMPLEMENTACIÓN Y PRUEBAS DE CAMPO DEL DIAGNOSTICADOR EN EL HC NO TRIPULADO.

Tabla 7.3: Tabla de Integración de Sensores - Adición de Lugares de Salida Motor.

\begin{tabular}{|c|c|c|c||c|c|}
\hline \multicolumn{7}{|l|}{ Lecturas Sensores } & \multicolumn{2}{l|}{ Lugares } \\
\hline$F$ & $T$ & $R P M$ & $I_{1}$ & $P 0(F, \bar{T}, R P M, \bar{I})$ & $P 1(F, \bar{T}, R P M, \bar{I})$ \\
\hline \hline $\bar{F}$ & $\bar{T}$ & $\overline{R P M}$ & $\overline{I_{1}}$ & & \\
\hline $\bar{F}$ & $\bar{T}$ & $\overline{R P M}$ & $I_{1}$ & & \\
\hline $\bar{F}$ & $\bar{T}$ & $R P M$ & $\overline{I_{1}}$ & & \\
\hline $\bar{F}$ & $\bar{T}$ & $R P M$ & $I_{1}$ & & \\
\hline $\bar{F}$ & $T$ & $\overline{R P M}$ & $\overline{I_{1}}$ & & \\
\hline $\bar{F}$ & $T$ & $\overline{R P M}$ & $I_{1}$ & & \\
\hline $\bar{F}$ & $T$ & $R P M$ & $\overline{I_{1}}$ & & \\
\hline $\bar{F}$ & $T$ & $R P M$ & $I_{1}$ & & \\
\hline$F$ & $\bar{T}$ & $\overline{R P M}$ & $\overline{I_{1}}$ & & \\
\hline$F$ & $\bar{T}$ & $\overline{R P M}$ & $I_{1}$ & & \\
\hline$F$ & $\bar{T}$ & $R P M$ & $\overline{I_{1}}$ & & \\
\hline$F$ & $\bar{T}$ & $R P M$ & $I_{1}$ & & \\
\hline$F$ & $T$ & $\overline{R P M}$ & $\bar{I}_{1}$ & & \\
\hline$F$ & $T$ & $\overline{R P M}$ & $I_{1}$ & & \\
\hline$F$ & $T$ & $R P M$ & $\overline{I_{1}}$ & & \\
\hline$F$ & $T$ & $R P M$ & $I_{1}$ & & \\
\hline
\end{tabular}

Tabla 7.4: Tabla de Integración de Sensores - Adición de Lugares de Salida Rotor Ppal.

\begin{tabular}{|c|c|c|c|c|c|c|}
\hline \multicolumn{9}{|c|}{} & \multicolumn{2}{c|}{ Lugares } \\
\hline$I_{S}$ & $R l$ & $P t$ & $G$ & $P 2\left(\overline{I_{S} R l}, \overline{P t}, \bar{G}\right)$ & $P 3\left(\overline{I_{S}} R l, \overline{P t}, \bar{G}\right)$ \\
\hline \hline$\overline{I_{S}}$ & $\overline{R l}$ & $\overline{P t}$ & $\bar{G}$ & & \\
\hline$\overline{I_{S}}$ & $\overline{R l}$ & $\overline{P t}$ & $G$ & & \\
\hline$\overline{I_{S}}$ & $\overline{R l}$ & $P t$ & $\bar{G}$ & & \\
\hline$\overline{I_{S}}$ & $\overline{R l}$ & $P t$ & $G$ & & \\
\hline$\overline{I_{S}}$ & $R l$ & $\overline{P t}$ & $\bar{G}$ & & \\
\hline $\bar{I}_{S}$ & $R l$ & $\overline{P t}$ & $G$ & & \\
\hline$\overline{I_{S}}$ & $R l$ & $P t$ & $\bar{G}$ & & \\
\hline$\overline{I_{S}}$ & $R l$ & $P t$ & $G$ & & \\
\hline$I_{S}$ & $\overline{R l}$ & $\overline{P t}$ & $\bar{G}$ & & \\
\hline$I_{S}$ & $\overline{R l}$ & $\overline{P t}$ & $G$ & & \\
\hline$I_{S}$ & $\overline{R l}$ & $P t$ & $\bar{G}$ & & \\
\hline$I_{S}$ & $\overline{R l}$ & $P t$ & $G$ & & \\
\hline$I_{S}$ & $R l$ & $\overline{P t}$ & $\bar{G}$ & & \\
\hline$I_{S}$ & $R l$ & $\overline{P t}$ & $G$ & & \\
\hline$I_{S}$ & $R l$ & $P t$ & $\bar{G}$ & & \\
\hline$I_{S}$ & $R l$ & $P t$ & $G$ & & \\
\hline
\end{tabular}


CAPÍTULO 7. IMPLEMENTACIÓN Y PRUEBAS DE CAMPO DEL DIAGNOSTICADOR EN EL HC NO TRIPULADO.

Tabla 7.5: Tabla de Integración de Sensores - Adición de Lugares de Salida Rotor Cola.

\begin{tabular}{|c|c||c|c|}
\hline \multicolumn{2}{|c|}{ Lect. Sens } & \multicolumn{2}{c|}{ Lugares } \\
\hline$I_{6}$ & $Y w$ & $P 4\left(\bar{I}_{6}, \overline{Y w}\right)$ & $P 5\left(\bar{I}_{6}, \overline{Y w}\right)$ \\
\hline \hline$\overline{I_{6}}$ & $Y w$ & & \\
\hline$\overline{I_{6}}$ & $\overline{Y w}$ & & \\
\hline$I_{6}$ & $Y w$ & & \\
\hline$I_{6}$ & $\overline{Y w}$ & & \\
\hline
\end{tabular}

cruzada con su correspondiente lectura de funcionamiento normal del lugar de cada subsistema. Las lecturas de los sensores, permiten identificar que lectura de los sensores es normal $N$, es fallo $F$ o es una lectura que brinda una información no útil $X$. Este paso requiere un profundo conocimiento del sistema. En el subsistema del Motor se usa la información de cada sensor, solo o en unión con los otros sensores para determinar los posibles fallos, por ello, estando el subsistema en el lugar $P 0$, sus lecturas normales son $F, \bar{T}, R P M, \bar{I}$. Si en algún momento estas lecturas cambian, mostrando que haya a que no haya combustible $\bar{F}$ por ejemplo, esto significa que ha ocurrido un fallo de nivel de combustible $F N C$, por el contrario, cuando las lecturas de los sensores coinciden con las esperadas, se registra en la tabla como un evento normal. Del mismo modo se realizan todas las combinaciones y lecturas para con los demás sensores. Esto se puede evidenciar en la tabla 7.6. La tabla de integración de sensores del rotor principal se muestra en la tabla 7.7 y el del subsistema de cola en la tabla 7.8.

- Paso 4.5. Reemplazar las transiciones de Fallo y Eliminar luGaRes de Fallo no Alcanzables. Este paso consiste en reemplazar transiciones no observables (transiciones de fallo) por lecturas sensoriales identificadas en la tabla de integración.

- Subsistema del Motor. En el modelo de RdP, se procede a sustituir la transición de $F N C$ por la lectura del sensor de flujo $F$, la transición de $F C M$ por la lectura de temperatura $T$ y la transición de $F A S 1$ por las lecturas de $R P M$ y $I_{1}$. La nueva RdP refinada del modelo del motor, se puede observar en la figura 7.27. 
Tabla 7.6: Tabla de Integración de Sensores - Motor.

\begin{tabular}{|c|c|c|c|c|c|c|c|c|c|c|c|}
\hline \multicolumn{4}{|c|}{ Lecturas Sensores } & \multicolumn{8}{|c|}{ Lugares } \\
\hline$F$ & $T$ & $R P M$ & $I_{1}$ & \multicolumn{4}{|c|}{$P 0\left(F, \bar{T}, R P M, \overline{I_{1}}\right)$} & \multicolumn{4}{|c|}{$\overline{P 1}\left(F, \bar{T}, R P M, \overline{I_{1}}\right)$} \\
\hline$\overline{\bar{F}}$ & $\bar{T}$ & $\overline{\overline{R P M}}$ & $\overline{\overline{I_{1}}}$ & $F N C$ & $\bar{N}$ & $F A S 1$ & $N$ & $F N C$ & $N$ & $F A S 1$ & $N$ \\
\hline $\bar{F}$ & $\bar{T}$ & $\overline{R P M}$ & $I_{1}$ & $F N C$ & $N$ & $F A S 1$ & $F A S 1$ & $F N C$ & $N$ & $F A S 1$ & $F A S 1$ \\
\hline $\bar{F}$ & $\bar{T}$ & $R P M$ & $\overline{I_{1}}$ & $F N C$ & $N$ & $N$ & $N$ & $F N C$ & $N$ & $N$ & $N$ \\
\hline $\bar{F}$ & $\bar{T}$ & $R P M$ & $I_{1}$ & $F N C$ & $N$ & $N$ & $F A S 1$ & $F N C$ & $N$ & $N$ & $F A S 1$ \\
\hline $\bar{F}$ & $T$ & $\overline{R P M}$ & $\overline{I_{1}}$ & $F N C$ & $F C M$ & $F A S 1$ & $N$ & $F N C$ & $F C M$ & $F A S 1$ & $N$ \\
\hline $\bar{F}$ & $T$ & $\overline{R P M}$ & $I_{1}$ & $F N C$ & $F C M$ & $F A S 1$ & $F A S 1$ & $F N C$ & $F C M$ & $F A S 1$ & $F A S 1$ \\
\hline $\bar{F}$ & $T$ & $R P M$ & $\overline{I_{1}}$ & $F N C$ & $F C M$ & $N$ & $N$ & $F N C$ & $F C M$ & $N$ & $N$ \\
\hline $\bar{F}$ & $T$ & $R P M$ & $I_{1}$ & $F N C$ & $F C M$ & $N$ & $F A S 1$ & $F N C$ & $F C M$ & $N$ & $F A S 1$ \\
\hline$F$ & $\bar{T}$ & $\overline{R P M}$ & $\overline{I_{1}}$ & $N$ & $N$ & $F A S 1$ & $N$ & $N$ & $N$ & $F A S 1$ & $N$ \\
\hline$F$ & $\bar{T}$ & $\overline{R P M}$ & $I_{1}$ & $N$ & $N$ & $F A S 1$ & $F A S 1$ & $N$ & $N$ & $F A S 1$ & $F A S 1$ \\
\hline$F$ & $\bar{T}$ & $R P M$ & $\overline{I_{1}}$ & $N$ & $N$ & $N$ & $N$ & $N$ & $N$ & $N$ & $N$ \\
\hline$F$ & $\bar{T}$ & $R P M$ & $I_{1}$ & $N$ & $N$ & $N$ & $F A S 1$ & $N$ & $N$ & $N$ & $F A S 1$ \\
\hline$F$ & $T$ & $\overline{R P M}$ & $\overline{I_{1}}$ & $N$ & $F C M$ & $F A S 1$ & $N$ & $N$ & $F C M$ & $F A S 1$ & $N$ \\
\hline$F$ & $T$ & $\overline{R P M}$ & $I_{1}$ & $N$ & $F C M$ & $F A S 1$ & $F A S 1$ & $N$ & $F C M$ & $F A S 1$ & $F A S 1$ \\
\hline$F$ & $T$ & $R P M$ & $\overline{I_{1}}$ & $N$ & $F C M$ & $N$ & $N$ & $N$ & $F C M$ & $N$ & $N$ \\
\hline$F$ & $T$ & $R P M$ & $I_{1}$ & $N$ & $F C M$ & $N$ & $F A S 1$ & $N$ & $\overline{F C M}$ & $N$ & $F A S 1$ \\
\hline
\end{tabular}

Tabla 7.7: Tabla de Integración de Sensores - Plato.

\begin{tabular}{|c|c|c|c||c|c|c|c||c|c|c|c|}
\hline$I_{S}$ & $R l$ & $P t$ & $G$ & \multicolumn{3}{|c||}{$P 2\left(\overline{I_{S}} \overline{R l}, \overline{P t}, \bar{G}\right)$} & \multicolumn{3}{|c|}{$P 3\left(\overline{I_{S}} \overline{R l}, \overline{P t}, \bar{G}\right)$} \\
\hline \hline$\overline{I_{S}}$ & $\overline{R l}$ & $\overline{P t}$ & $\bar{G}$ & $N$ & $N$ & $N$ & $N$ & $N$ & $N$ & $N$ & $N$ \\
\hline \hline$\overline{I_{S}}$ & $\overline{R l}$ & $\overline{P t}$ & $G$ & $N$ & $N$ & $N$ & $F M$ & $N$ & $N$ & $N$ & $F M$ \\
\hline$\overline{I_{S}}$ & $\overline{R l}$ & $P t$ & $\bar{G}$ & $N$ & $N$ & $F A S$ & $N$ & $N$ & $N$ & $F A S$ & $N$ \\
\hline$\overline{I_{S}}$ & $\overline{R l}$ & $P t$ & $G$ & $N$ & $N$ & $F A S$ & $F M$ & $N$ & $N$ & $F A S$ & $F M$ \\
\hline$\overline{I_{S}}$ & $R l$ & $\overline{P t}$ & $\bar{G}$ & $N$ & $F A S$ & $N$ & $N$ & $N$ & $F A S$ & $N$ & $N$ \\
\hline$\overline{I_{S}}$ & $R l$ & $\overline{P t}$ & $G$ & $N$ & $F A S$ & $N$ & $F M$ & $N$ & $F A S$ & $N$ & $F M$ \\
\hline$\overline{I_{S}}$ & $R l$ & $P t$ & $\bar{G}$ & $N$ & $F A S$ & $F A S$ & $N$ & $N$ & $F A S$ & $F A S$ & $N$ \\
\hline$\overline{I_{S}}$ & $R l$ & $P t$ & $G$ & $N$ & $F A S$ & $F A S$ & $F M$ & $N$ & $F A S$ & $F A S$ & $F M$ \\
\hline$I_{S}$ & $\overline{R l}$ & $\overline{P t}$ & $\bar{G}$ & $F A S$ & $N$ & $N$ & $N$ & $F A S$ & $N$ & $N$ & $N$ \\
\hline$I_{S}$ & $\overline{R l}$ & $\overline{P t}$ & $G$ & $F A S$ & $N$ & $N$ & $F M$ & $F A S$ & $N$ & $N$ & $F M$ \\
\hline$I_{S}$ & $\overline{R l}$ & $P t$ & $\bar{G}$ & $F A S$ & $N$ & $F A S$ & $N$ & $F A S$ & $N$ & $F A S$ & $N$ \\
\hline$I_{S}$ & $\overline{R l}$ & $P t$ & $G$ & $F A S$ & $N$ & $F A S$ & $F M$ & $F A S$ & $N$ & $F A S$ & $F M$ \\
\hline$I_{S}$ & $R l$ & $\overline{P t}$ & $\bar{G}$ & $F A S$ & $F A S$ & $N$ & $N$ & $F A S$ & $F A S$ & $N$ & $N$ \\
\hline$I_{S}$ & $R l$ & $\overline{P t}$ & $G$ & $F A S$ & $F A S$ & $N$ & $F M$ & $F A S$ & $F A S$ & $N$ & $F M$ \\
\hline$I_{S}$ & $R l$ & $P t$ & $\bar{G}$ & $F A S$ & $F A S$ & $F A S$ & $N$ & $F A S$ & $F A S$ & $F A S$ & $N$ \\
\hline$I_{S}$ & $R l$ & $P t$ & $G$ & $F A S$ & $F A S$ & $F A S$ & $F M$ & $F A S$ & $F A S$ & $F A S$ & $F M$ \\
\hline
\end{tabular}


Tabla 7.8: Tabla de Integración de Sensores del Rotor de Cola.

\begin{tabular}{|c|c||c|c|}
\hline$I_{6}$ & $Y w$ & $P 5\left(\bar{I}_{6}, \overline{Y w}\right)$ & $P 6\left(\bar{I}_{6}, \overline{Y w}\right)$ \\
\hline \hline $\bar{I}_{6}$ & $Y w$ & $N$ & $N$ \\
\hline $\bar{I}_{6}$ & $\overline{Y w}$ & $F A S 6$ & $F A S 6$ \\
\hline$I_{6}$ & $Y w$ & $F A S 6$ & $F A S 6$ \\
\hline$I_{6}$ & $\overline{Y w}$ & $F A S 6$ & $F A S 6$ \\
\hline
\end{tabular}

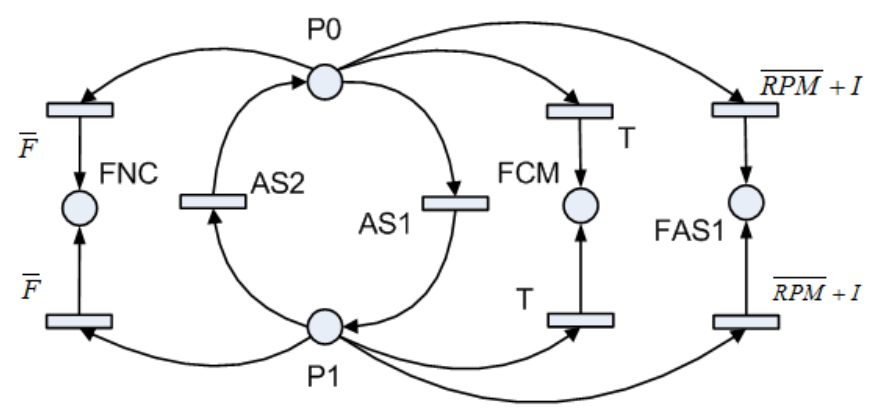

Figura 7.27: Figura 16. Modelo Refinado de RdP del Subsistema del Motor

- Subsistema del Rotor Principal. El subsistema del rotor depende del funcionamiento de los cuatro servos que componen el plato, por ello, en el modelo de RdP, se procede a sustituir las transiciones de $F A S 2$, $F A S 3, F A S 4, F A S 5$ por las lecturas respectivas de los sensores de corrientes de servos $I_{2}, I_{3}, I_{4}, I_{5}$ y el fallo mecánico $F M$ es reemplazado por $G$; además, encontramos que la lectura de los sensores de ángulos roll $R l$ y pitch $P t$ me indican un fallo de $F A S$ y que la lectura de las vibraciones me indican un fallo mecánico $F M$, asumiendo que puede estar en fallo cualquiera de los servos del rotor principal. La nueva $\mathrm{RdP}$ refinada del modelo del rotor, se puede observar en la figura 7.28 .

- Subsistema del Rotor de Cola. En el modelo de RdP refinado de la figura 7.29, se ha sustituido el $F A S 6$ por las lecturas de $I_{6}$ y la lectura del angulo Yaw $Y w$.

\section{Paso 5. Construcción del Diagnosticador.}

Finalmente, todo la de herramienta está integrada en una sola RdP para el FD del helicóptero. La RdP que representa el diagnosticador se compone principalmente de tres ramas, correspondiente a cada subsistema: motor, ramas 


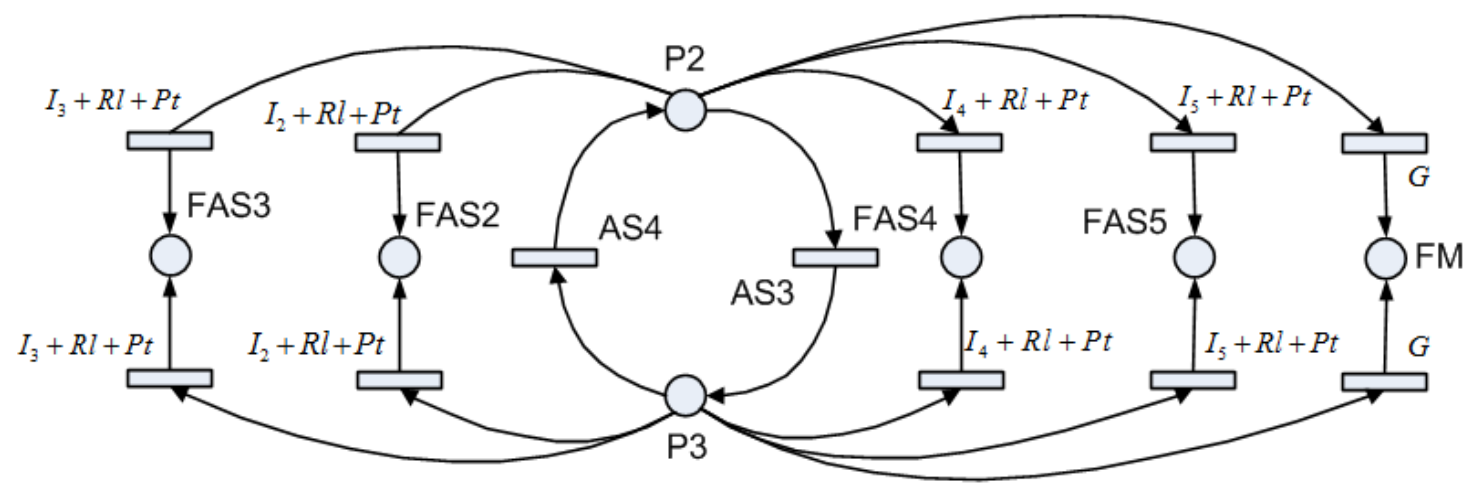

Figura 7.28: Modelo Refinado de RdP del Subsistema del Rotor Principal

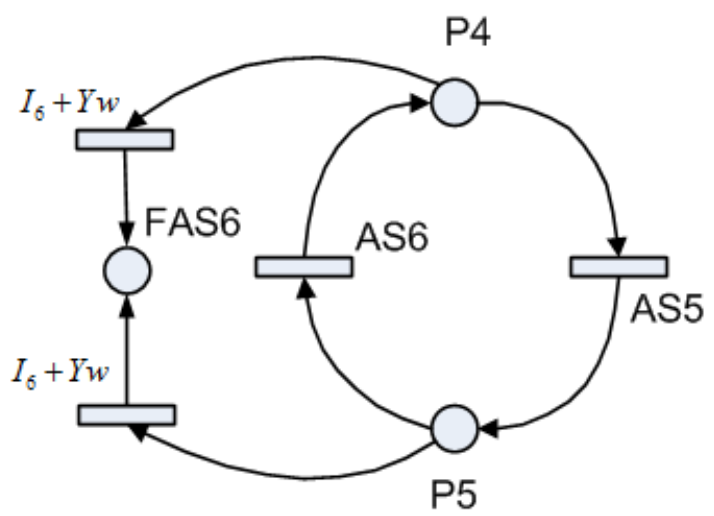

Figura 7.29: Modelo Refinado de RdP del Subsistema del Rotor de Cola 
del rotor principal y del rotor de cola, como la figura 7.30 describe. Un lugar de inicio $p_{0}$ y una transición de inicio $t_{0}$ para el inicio del diagnosticador han sido definidos. La transición de inicio $t_{0}$ tiene que ser activada manualmente por el operador en la estación de control de tierra para iniciar el diagnosticador. Ésta mueve una marca a cada una de las ramas de los subsistemas del helicóptero. Asimismo, la RdP diagnosticadora tiene una transición de finalización $t_{\text {end }}$, que permite al piloto finalizar el diagnosticador. Los modelos RdP refinados de cada subsistema están unidos al diagnosticador con sus respectivos lugares y transiciones; seguido basado en la función de expansión de fallo $E F$, las ramas de fallo son adicionadas a cada subsistema. Las transiciones de recuperación $t_{R}$ de cada uno de los lugares de fallo de cada subsistema son adicionados, conectando con el lugar normal previo. Después, Cada uno de los lugares y transiciones deben ser conectados por arcos, teniendo en cuenta el peso definido por el número de fallos más uno. Finalmente, la dinámica temporal debe ser implementada adicionando los lugares de conteo temporal $p_{C}$, el cual es usado para almacenar el tiempo y el número de fallos de cada variable del sistema.

El diagnosticador hace una evaluación del sistema completo y sirve como supervisor, indicando si una rama cae en fallo por medio de la función de asignación de etiquetas $L A$. Si esto pasa, las otras ramas continúan evaluando el sistema, aunque, debido a la vulnerabilidad del helicóptero, éste debe ser aterrizado para ser reparado inmediatamente.

Algunas veces, pequeños fallos pasan durante un vuelo por cortos periodos de tempo. Si ninguna acción adicional es suministrada, las transiciones de recuperación ocultaran estos fallos al operador. El diagnosticador se apoya en una pantalla para mostrar al operador este tipo de información.

\subsubsection{Análisis de Resultados.}

Usando el sistema de adquisición da datos visto en la sección 7.0.2, diseñado e implementado para las pruebas de la herramienta de FD, se han realizado una serie de misiones en campo, que han permitido evaluar el comportamiento de la herramienta. Estas evaluaciones han pasado por pruebas de laboratorio para evaluar el sistema DAQ, y vuelos de la aeronave para poder establecer los umbrales de las variables en funcionamiento normal, por último, asumiendo el riesgo que implica la misión, se han realizado misiones genera- 


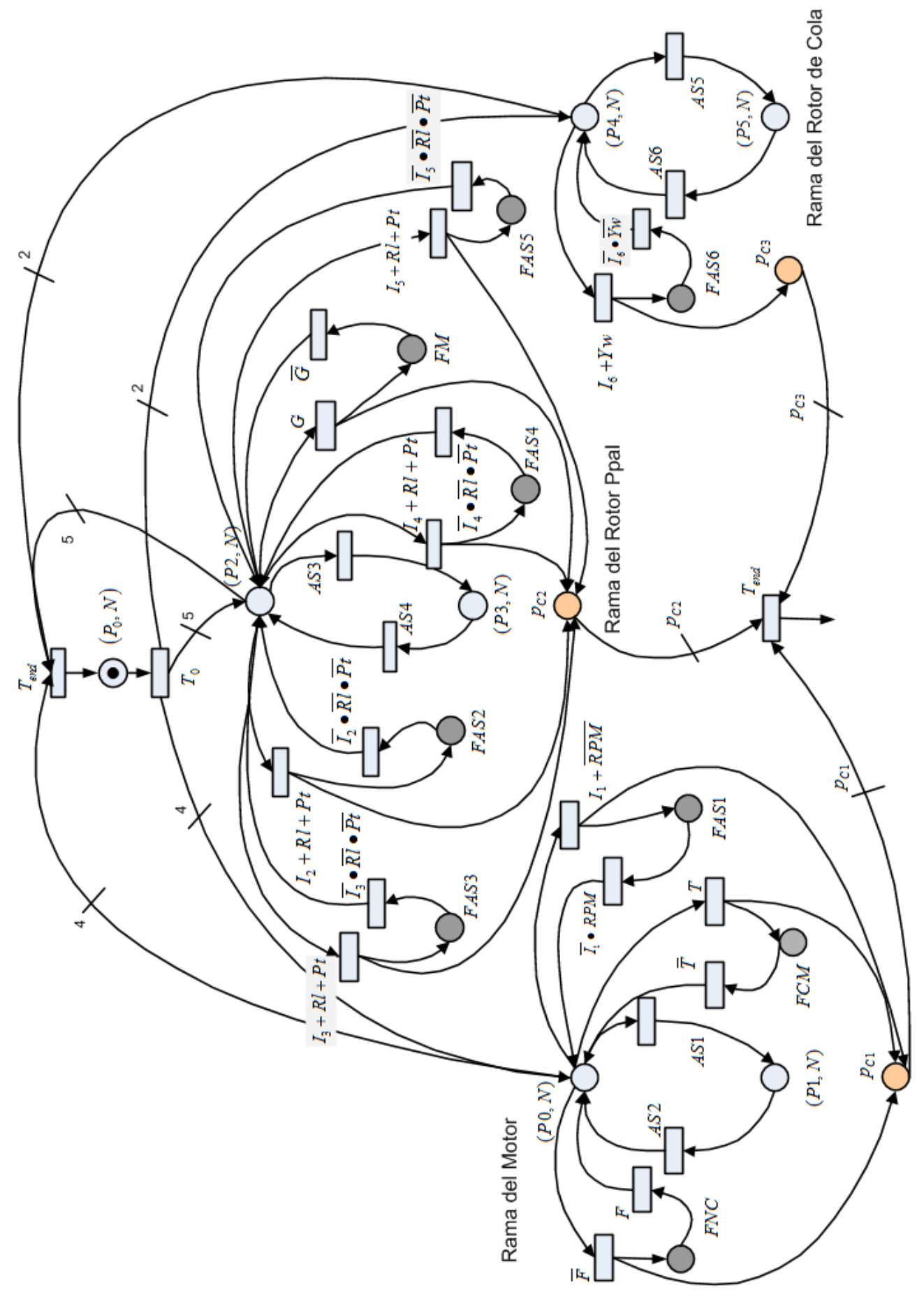

Figura 7.30: Diagnosticador del Helicóptero 
CAPÍTULO 7. IMPLEMENTACIÓN Y PRUEBAS DE CAMPO DEL DIAGNOSTICADOR EN EL HC NO TRIPULADO.

Tabla 7.9: Resumen de Misiones

\begin{tabular}{|c|c|c|c|c|}
\hline Fecha & Lugar & Hora & duración & observaciones \\
\hline \hline $12-12-2010$ & Laboratorio & $15: 11$ & $2: 30$ & Validación De DAQ \\
\hline $12-02-2011$ & Laboratorio & $17: 19$ & $1: 30$ & Validación De DAQ \\
\hline $13-02-2011$ & Laboratorio & $15: 10$ & $2: 20$ & Validación De DAQ \\
\hline $18-02-2011$ & Laboratorio & $13: 18$ & $8: 30$ & Validación De DAQ \\
\hline $20-02-2011$ & Laboratorio & $19: 29$ & $2: 20$ & Validación De DAQ \\
\hline $25-02-2011$ & Laboratorio & $11: 28$ & $7: 15$ & Validación De DAQ \\
\hline $27-02-2011$ & Campo de Vuelo & $18: 16$ & $2: 00$ & Recolección de datos \\
\hline $03-02-2013$ & Campo de Vuelo & $16: 57$ & $2: 00$ & Ajuste y Rec.de datos \\
\hline $10-02-2013$ & Campo de Vuelo & $16: 38$ & $2: 00$ & Ajuste y Rec. de datos \\
\hline $23-03-2013$ & Campo de Vuelo & $16: 30$ & $3: 00$ & Rec. de datos y fallos \\
\hline $07-10-2013$ & Laboratorio & $18: 00$ & $4: 00$ & Ajustes Electrónica \\
\hline $17-10-2013$ & Campo de Vuelo & $16: 30$ & $3: 00$ & Rec. de datos y fallos \\
\hline $15-02-2014$ & Campo de Vuelo & $15: 30$ & $2: 00$ & Recolección de datos \\
\hline $18-03-2014$ & Campo de Vuelo & $16: 00$ & $2: 00$ & Recolección de datos \\
\hline
\end{tabular}

do fallos en la aeronave con el fin de validar la herramienta, estas pruebas se detallan a continuación en la tabla 7.9:

Para la implementación de la herramienta de FD, era necesario conocer los parámetros de funcionamiento normal de la aeronave. Debido a que no se conocen datos sobre las variables del helicóptero, una de las primeras tareas en este proceso, fue la de establecer los rangos de medida de las variables de la aeronave de funcionamiento normal y de estado de alarma. Previamente a esta tarea, se validó en el laboratorio que las medidas recibidas en la RdP diagnosticadora, implementada en LabVIEW, coincidieran con las medidas reales. Una vez las medidas eran las óptimas y validas de manera real, se realizan vuelos de funcionamiento normal para conocer el comportamiento de la aeronave, y por último, se generan dos fallos buscando que la herramienta mostrara el comportamiento anormal de la misión. Los fallos generados fueron: aumento de vibraciones en el rotor principal y cola, y daño de la mezcla (aire/gas) del motor, es decir se restringió el paso de gas en el carburador. A continuación se detallan las conclusiones observadas en cada una de las variables medidas. 
CAPÍTULO 7. IMPLEMENTACIÓN Y PRUEBAS DE CAMPO DEL DIAGNOSTICADOR EN EL HC NO TRIPULADO.

\section{Reglas para definir los Estados de Alarma o Fallo}

Después de la construcción del RdP diagnosticadora, es necesario definir las reglas que permiten determinar si puede existir un estado de advertencia o fallo. Estas reglas se basan en la experiencia de un piloto experto, que es capaz de asociar los cambios en las variables con sus causas, ofreciendo al operador el apoyo para encontrar el problema y actuar en consecuencia. Estas reglas se definen mediante el uso de dos tipos de situaciones anormales:

- Advertencia. Se define cuando una variable excede un umbral de fallo durante un cierto período de tiempo (dependiendo de las variables, un valor de tres segundos se ha considerado para altas dinámicas). Si la variable vuelve a su valor normal antes de este tiempo de espera, no va a ser considerado como una advertencia.

- Fallo. Se define de acuerdo a las siguientes reglas:

- Cualquier alarma que se activa y se mantiene durante más de 5 segundos.

- Dos advertencias simultáneas se producen y se mantienen durante más de 3 segundos.

- 3 o más advertencias ocurren simultáneamente.

Cada variable, de forma aislada o junto con una o más variables se clasifica de acuerdo a su importancia en la operación de la aeronave. Las variables consideradas en el diagnosticador son:

- Voltaje de la batería $(V)$. Cualquier aumento en la tensión de umbral mínimo de voltaje se convierte en culpa.

$$
F_{1}=\bar{V}
$$

- Actitud del helicóptero $(\theta)$. (es decir, ángulos de balanceo, cabeceo y guiñada), así como las corrientes de servo $\left(I_{s}\right)$ son señales que pueden superar en cualquier momento su umbral de fallo. Las señales deben exceder su valor no más que el tiempo de espera establecido ( 3 segundos en este caso);

$$
F_{2}=(\varnothing \cdot t)+(I s \cdot t)
$$


Tabla 7.10: Tabla de Verdad de Interacción de la $T / F / R P M / G$

\begin{tabular}{|c|c|c|c|c|}
\hline$T$ & $F$ & $R P M$ & $G$ & Fallo \\
\hline \hline 0 & 0 & 0 & 0 & 1 \\
\hline 0 & 0 & 0 & 1 & 1 \\
\hline 0 & 0 & 1 & 0 & 1 \\
\hline 0 & 0 & 1 & 1 & 0 \\
\hline 0 & 1 & 0 & 0 & 1 \\
\hline 0 & 1 & 0 & 1 & 1 \\
\hline 0 & 1 & 1 & 0 & 1 \\
\hline 0 & 1 & 1 & 1 & 0 \\
\hline 1 & 0 & 0 & 0 & 1 \\
\hline 1 & 0 & 0 & 1 & 1 \\
\hline 1 & 0 & 1 & 0 & 1 \\
\hline 1 & 0 & 1 & 1 & 0 \\
\hline 1 & 1 & 0 & 0 & 1 \\
\hline 1 & 1 & 0 & 1 & 0 \\
\hline 1 & 1 & 1 & 0 & 0 \\
\hline 1 & 1 & 1 & 1 & 0 \\
\hline
\end{tabular}

- Temperatura $(T)$, flujo de combustible $(F)$, revoluciones por minuto $(R P M)$ y Vibraciones $(G)$, están fuertemente relacionados entre sí en el helicóptero, por lo que requieren un análisis global en lugar de uno independiente. Una tabla de verdad (tabla 7.10) se ha construido con el fin de definir un conjunto o reglas que se utilizarán para determinar si el sistema cae en fallo o no. Una excepción se considera en lo que se refiere esta regla: En el caso de que el combustible y las variables de temperatura superan su umbral de fallo a la vez, una advertencia se activa.

De acuerdo con las condiciones de fallo definidos en $F 1, F 2$ y $F 3$, una condición de fallo global de $F$ puede ser creada usando el operador OR:

Basados en la técnica de mapas de Kanaugh, sacamos la ecuación que nos define el fallo en su mínima expresión:

$$
F_{3}=\bar{G}(\bar{T}+\bar{F}+\overline{R P M})+\overline{R P M}(\bar{T}+\bar{F})
$$

Por último, hacemos una una unión de las tres condiciones de fallo vistas anteriormente a $F$ 


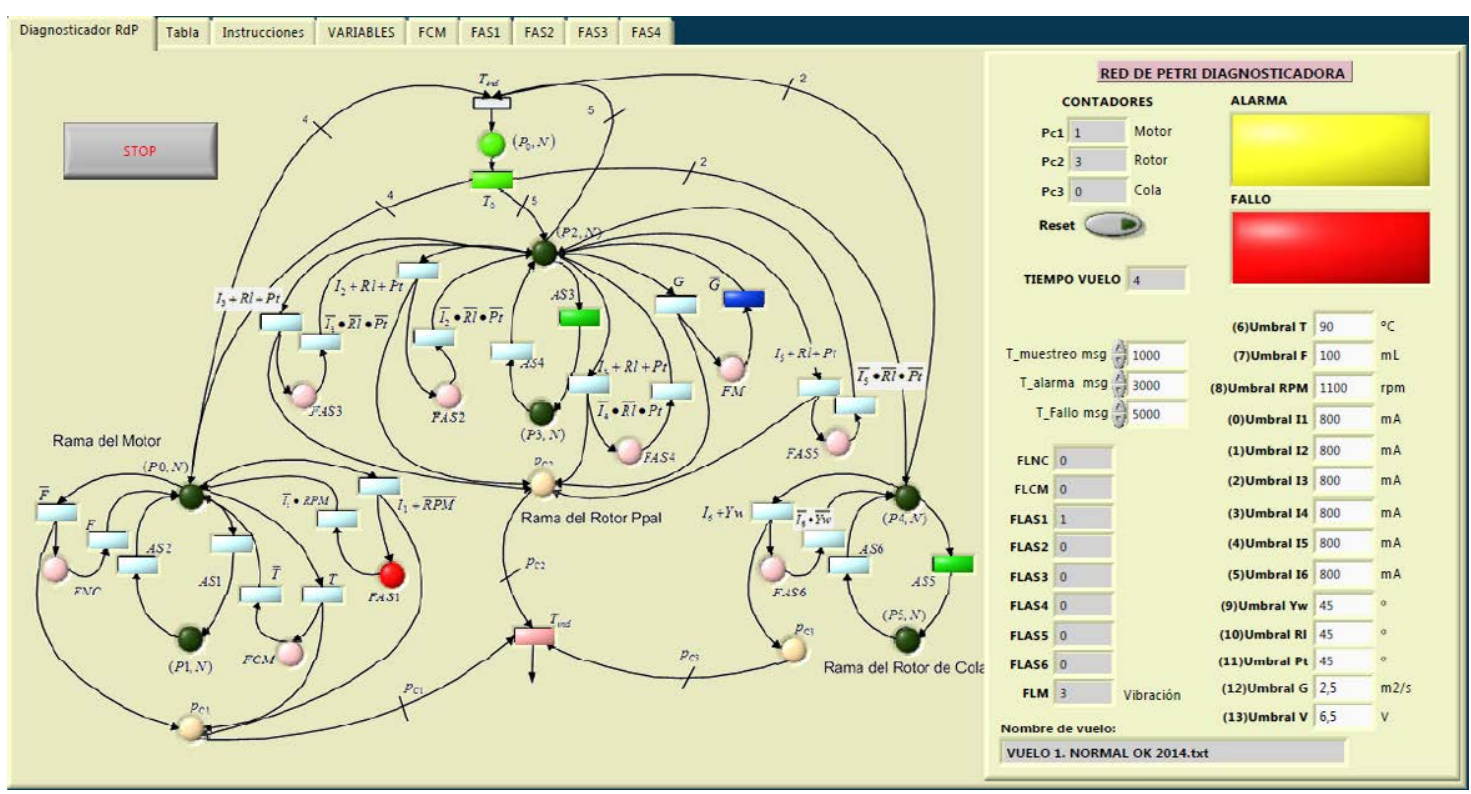

Figura 7.31: Red de Petri Diagnosticadora

$$
F=\bar{V}+(\varnothing \cdot t)+(I s \cdot t)+\bar{G}(\bar{T}+\bar{F}+\overline{R P M})+\overline{R P M}(\bar{T}+\bar{F})
$$

Como se describió en la sección 7.0.2, la herramienta de software construida en LabVIEW y quien se encarga de representar el diagnosticador, se le ha adicionado las condiciones de alarma y fallo, descritas anteriormente, para que en tiempo real cuando se esté realizando un vuelo, el piloto de la aeronave tenga la información on-line de la salud de la misión y pueda tomar las decisiones adecuadas de abortar la misión o continuar volando. En la siguiente figura se puede ver el RdP diagnosticadora que ofrece la información de la misión, funcionando en tiempo real y aislando cualquier variable que cae en fallo.

\section{Análisis del Comportamiento de las Variables Monitoreadas Durante los} Vuelos del Helicóptero TEMPERATURA. En la figura 7.32 podemos ver el comportamiento de las temperaturas recogidas en las misiones desarrolladas, tanto en situaciones de fallo como en estado normal. La temperatura del motor mantiene un valor entre la temperatura ambiente y un máximo de $90^{\circ} \mathrm{C}$ durante el funcionamiento normal, con una creciente estándar de 2,1 $\stackrel{\circ}{\circ} /$ seg transitorio, aunque este valor depende ligeramente del medio ambiente en el que la misión 


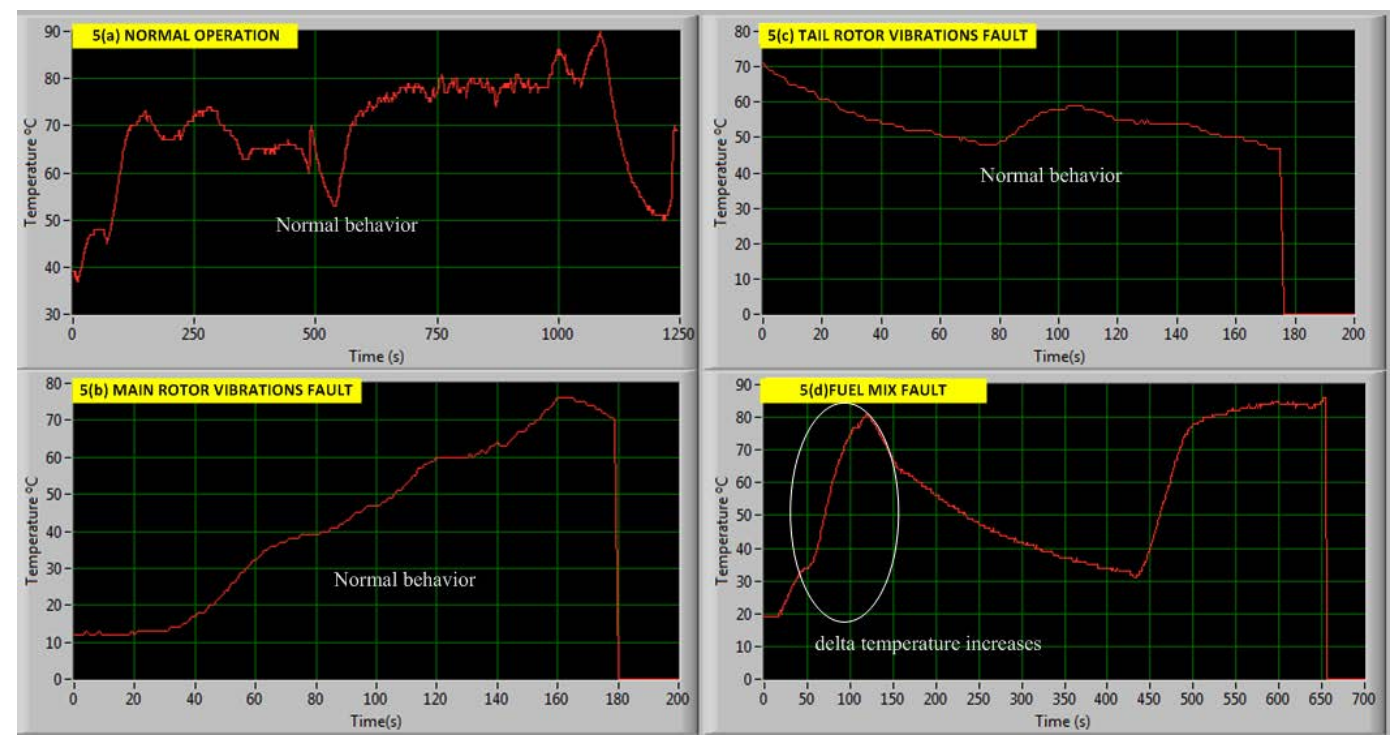

Figura 7.32: Resumen Temperaturas vuelos

se lleva a cabo. Cualquiera de los valores más altos o más bajos revelan problemas durante el vuelo. Por lo tanto, un valor por debajo del umbral normalmente indica que el motor podría estar recibiendo más inyección de gas de lo necesario, sino por el contrario (es decir, un valor más alto) indica problemas en el motor, como la mala combustión. Una mala combustión puede tener diferentes causas, tales como pérdida de sincronización en la detonación, no carburación óptima (bajo gas) y, en algunos casos, un mal ajuste de la potencia del motor con respecto al paso de las palas (configuración incorrecta de las palas de compensación en el emisor RC). En esta situación (es decir, el límite excedido) la misión debe ser abortado.

Por otra parte, teniendo en cuenta las condiciones de fallo generados en los vuelos de prueba, se demostró que el delta de la temperatura aumenta cuando se genera el fallo en la mezcla, como se destaca en la figura 7.32, pero mantiene valores normales cuando la aeronave sufre de un fallo de la vibración.

VOLTAJE DE BATERÍA. Por lo general, dos sistemas de alimentación se utilizan en grandes vehículos aéreos no tripulados, es decir, una batería y un generador. Este generador, que es accionado por el motor principal, es responsable de generar la tensión del sistema. Además, un regulador de tensión que entrega múltiples salidas proporciona las diferentes tensiones a la aeronave. Cuando la aeronave no posee un generador, la batería asume su función y tiene que ser recargada antes de volar. La carga de la batería depende del tiempo de 
CAPÍTULO 7. IMPLEMENTACIÓN Y PRUEBAS DE CAMPO DEL DIAGNOSTICADOR EN EL HC NO TRIPULADO.

uso y el número de ciclos que ha sufrido previamente la carga. Durante los vuelos efectuados para la validación del sistema, el voltaje de la batería resultó ser bastante estable y en ningún caso mostró caídas de tensión. Un umbral de 6,5 voltios fue instalado en el sistema utilizado en este trabajo como voltaje mínimo permitido en operación. Este valor puede diferir dependiendo de la tecnología de batería utilizada y sus variaciones en la curva de descarga, por lo tanto, debe ser personalizado en cualquier caso.

FLUJO DE COMBUSTIBLE. EI helicóptero utilizado en este trabajo utiliza un tanque con una capacidad de $500 \mathrm{ml}$ aproximadamente, lo que permite volar aproximadamente 20 minutos. Este tiempo varía dependiendo de la carga útil y la altitud. Hay tres maneras de rastrear el almacenamiento y el consumo de combustible en el avión: mediante el uso de un sensor de bajo nivel (discreta), que sólo detecta que hay un nivel mínimo que garantice la seguridad de la misión; midiendo la presión en el circuito (esto permite la detección de una fuga en las mangueras de combustible y conexiones) y, finalmente, mediante la medición del flujo de combustible de una manera analógica. Esta última solución fue elegido en este trabajo sobre todo porque permite detectar más condiciones de mal funcionamiento que los anteriores. Por lo tanto, un sensor fue instalado en la tubería de suministro.

Las pruebas de vuelo han permitido definir un umbral de $100 \mathrm{ml}$ para el almacenamiento de combustible mínimo. Por otra parte, la prueba evaluó que el flujo de combustible se ve afectada de algún fallo del sistema motor. Por lo tanto, el consumo por lo general varía de manera significativa cuando el sistema requiere un gran esfuerzo al motor. De acuerdo con esta característica, un consumo medio de combustible de $0,50 \mathrm{~mL} / \mathrm{seg}$ se estableció con las condiciones normales de vuelo. Además, se estableció que se detecta un fallo en el sistema de motor si un consumo de más de $1 \mathrm{~mL} / \mathrm{seg}$ se mantiene durante más de 3 seg.

VELOCIDAD DEL ROTOR. Se mide generalmente contando las revoluciones del plato en un período de tiempo. Su valor puede variar entre límites superior e inferior. Una disminución constante en las revoluciones indica un fallo mecánico debido a cualquiera de las siguientes partes: motor, transmisión, engranajes o el embrague entre otros. Esta variable está dentro del rango de 1250-1450 RPM en vuelo normal para el helicóptero utilizado durante las pruebas. Sin embargo, la velocidad es superior a 2000 RPM durante el despegue 


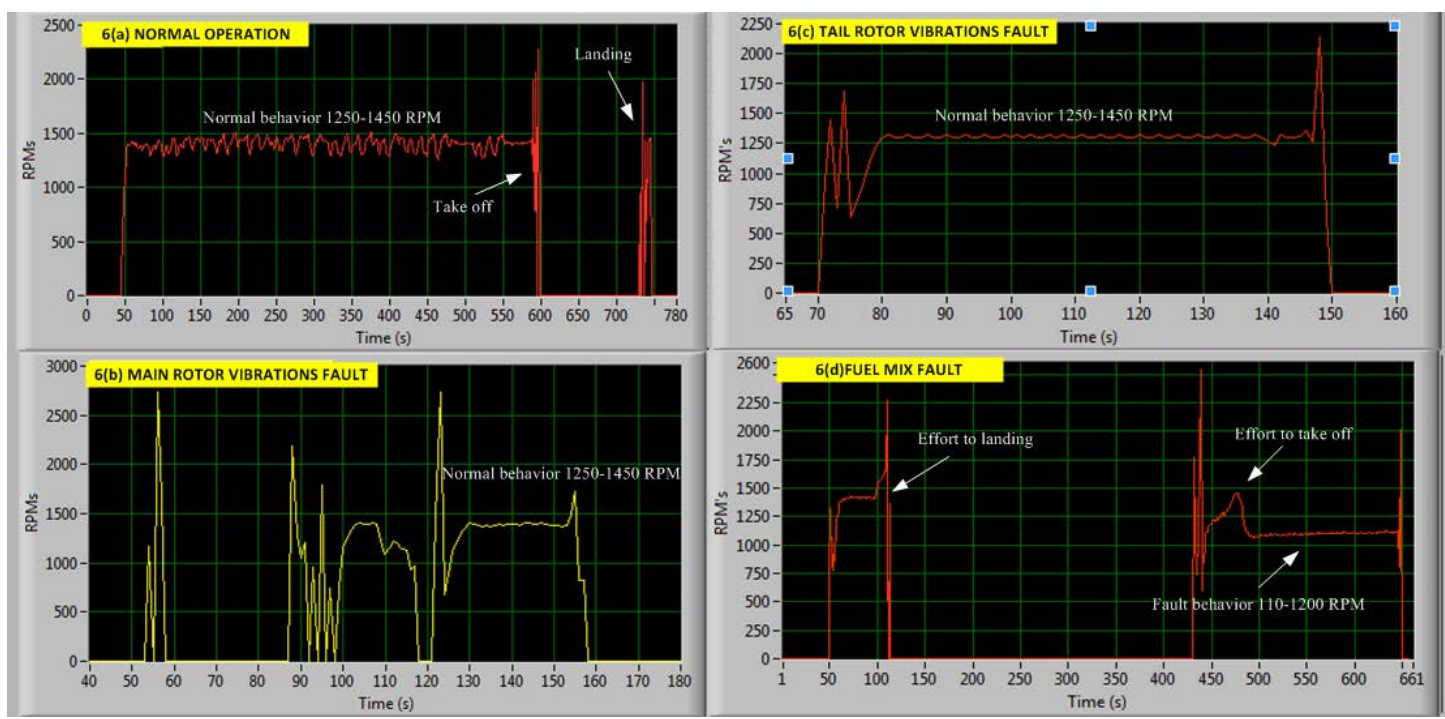

Figura 7.33: Revoluciones Vuelo Normal y Fallo del HC

y el aterrizaje de maniobras, como muestra la figura 7.33. En condiciones de fallo de vibración, el piloto necesita más tiempo para alcanzar la estabilidad de vuelo; Por lo tanto, el número de revoluciones se sale de su rango normal y oscila a una frecuencia más alta. Por otra parte, se puede observar que el motor hace un esfuerzo adicional, (es decir, el piloto tiene que aumentar la velocidad del motor) para llegar al vuelo normal. En el fallo de gas combustible, el rango puede variar durante el despegue a valores cercanos a 1400 RPM y cambia de 1.100 a 1.200 rpm una vez en vuelo como representa la figura 7.33.

VIBRACIONES. El comportamiento de las vibraciones de la estructura (chasis) depende en gran medida del tipo de maniobra (inactivo, despegue, aterrizaje, flotando, y el vuelo de traslación). Estas vibraciones se registran sus valores y se miden en amplitud. La amplitud se evalua en el eje vertical. Cuando la aeronave se apaga y no hay movimiento, esta amplitud muestra un valor constante de 1g, debido a la gravedad, mientras que el valor llega a ser mayor si la aeronave está en reposo (motor encendido y la aeronave en el suelo). Los valores durante vuelos normales (con amplitud cerca de $2 \mathrm{~g}$ ) son menores que cuando está en reposo (cerca de 2,5g). Sin embargo, un incremento considerable en la amplitud de las vibraciones se detecta, cuando un fallo de vibración es forzado en el rotor principal, con valores similares a los que cuando el HC está en reposo, alcanzando valores de $2,3 \mathrm{~g}$. Esta comparación de las amplitudes se 


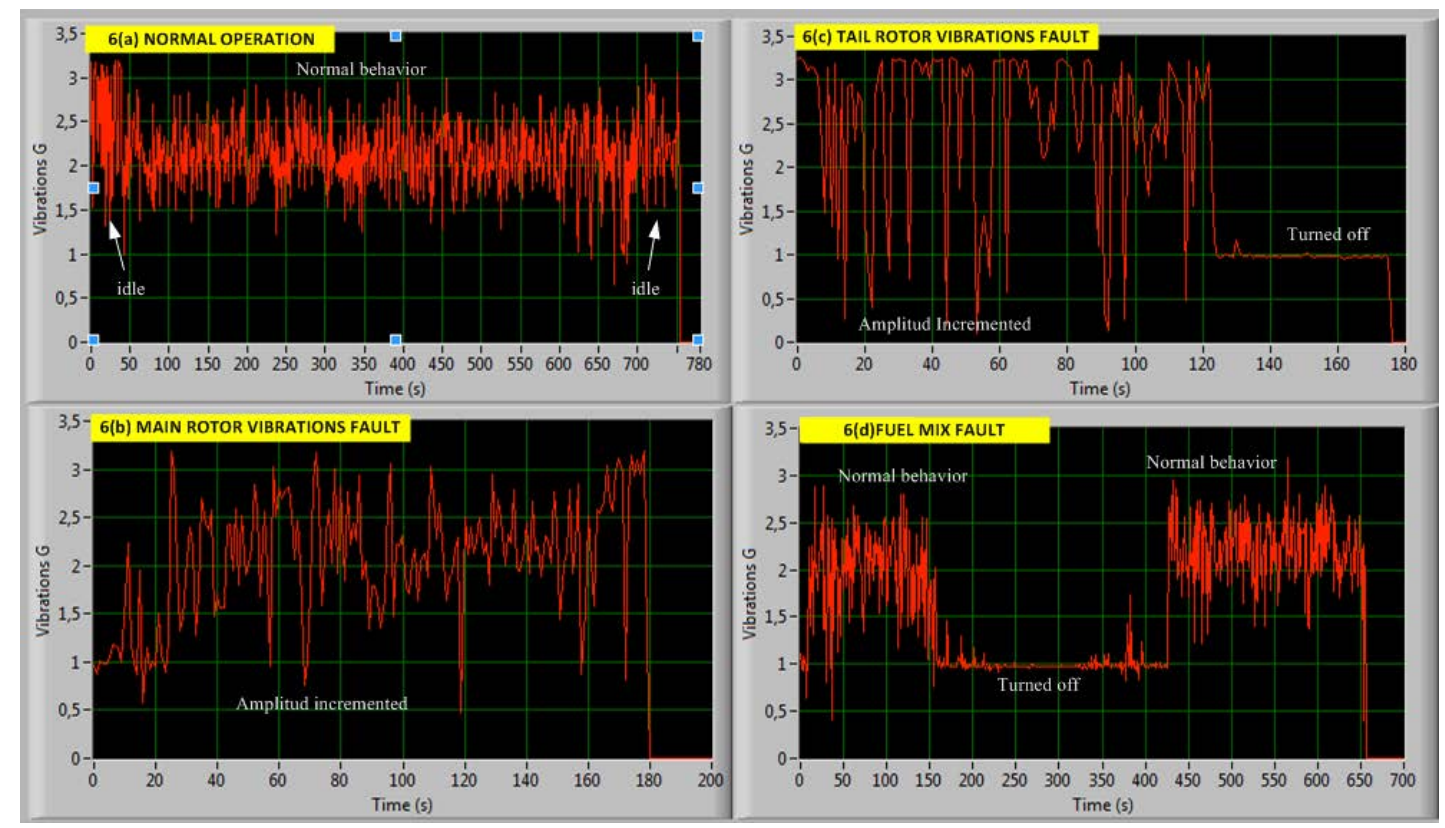

Figura 7.34: Comparación Vibraciones de Vuelo Normal y de Fallo

puede apreciar en la figura 7.34. En el fallo mezcla de combustible no afecta al comportamiento de las vibraciones en la aeronave.

CORRIENTE DE SERVOS. En la práctica, hay dos maneras de detectar si un servo está dañado, mediante la lectura de su consumo eléctrico, por lo tanto, si la corriente de servo es cero, la medición indica algún tipo de desconexión, mientras que un incremento constante del consumo por lo general indica que el servo no es capaz de llegar a la referencia comandado por el control. Esta situación generalmente pone de relieve una interferencia mecánica. Como se mencionó anteriormente, el consumo de cada uno de los seis servos se controla durante los vuelos. No se observaron cambios sustanciales en el consumo de corriente de los servos durante las pruebas, ni en vuelos normales, ni en los de fallo. El consumo normal de servos esta en el intervalo de 100 a 600 mA. Sin embargo, durante las misiones con fallo (por ejemplo, la vibración y la insuficiencia de combustible de la mezcla), se requieren más maniobras del piloto para estabilizar el helicóptero, causando un mayor número de cambios en el consumo de corriente de los servos.

ACTITUD DEL HELICÓPTERO. Ángulos de balanceo, cabeceo y guiñada están registrados con el fin de monitorear la actitud del helicóptero. Además, el emisor de radio usado durante los vuelos de prueba delimita los ángulos 
CAPÍTULO 7. IMPLEMENTACIÓN Y PRUEBAS DE CAMPO DEL DIAGNOSTICADOR EN EL HC NO TRIPULADO.

Tabla 7.11: Measured Variables and Thresholds in UAV

\begin{tabular}{|c|c|c|c|}
\hline Variable & Símbolo & Vr. Normal(1) & Vr Fallo (0) \\
\hline \hline Temperatura & $T$ & T. ambiente $-90^{\circ} \mathrm{C}$ & $100^{\circ} \mathrm{C}$ \\
\hline Voltaje & $V$ & $6,5-7 \mathrm{~V}$ & $0-6 \mathrm{~V}$ \\
\hline Flujo de Combustible & $F$ & $100-500 \mathrm{~mL}$ & $0-50 \mathrm{~mL}$ \\
\hline RPM's & $R P M$ & $1200-1500$ & $<1200$ \\
\hline Vibraciones & $G$ & $0,5-2,5 G$ & $>2,5 G$ \\
\hline Corriente de Servo & $I_{s}$ & $100-600 \mathrm{~mA}$ & $800 \mathrm{~mA}$ \\
\hline Ángulos RI/Pt/Yw & $\theta$ & $R l / P t / Y w<45^{\circ}$ & $R l / P t / Y w>45^{\circ}$ \\
\hline
\end{tabular}

de plato máximos permitidos, lo que limita los ángulos de balanceo y cabeceo esperados bajo un umbral de 45 grados. Por lo tanto, los valores más altos se consideran como un fallo.

Después de evaluar las variables mencionadas anteriormente en el avión durante los vuelos de prueba, se puede concluir que algunos de ellos (es decir, las vibraciones, la velocidad del rotor, el consumo de combustible y temperatura del motor) merecen una mención especial sobre los demás. Por otra parte, la seguridad del helicóptero depende en gran medida de su rendimiento mecánico, y la experiencia del piloto es el mejor indicador para decidir si una misión debe ser abortada o no.

Vale la pena señalar que la prueba requerida para evaluar el desempeño del diagnosticador es extremadamente arriesgada, ya que requieren forzar fallos reales durante los vuelos. En la tabla 7.11, se muestra un resumen de las variables consideradas durante las pruebas, incluyendo sus umbrales para determinar el comportamiento normal y fallo.

Algunas dificultades se encuentran comúnmente cuando se trabaja con sistemas de tiempo real que tienen que ser embarcados en un vehículo con una carga útil muy limitada (por ejemplo, las limitaciones de procesamiento de datos, el tamaño de la información y el consumo de elementos de procesamiento). Surgen problemas adicionales cuando la información debe ser transmitida a la estación de tierra (el ruido, el rango de las comunicaciones, ancho de banda limitado). Por lo tanto, es esencial ser tan pragmática como sea posible, es decir, usar el tiempo máximo de muestreo y las variables discretas esenciales. Otra limitación importante es el tiempo de reacción del piloto para decidir si una misión tiene que ser abortada cuando una advertencia/fallo se detecta, considerando las graves consecuencias que se producen cuando un avión se estrella contra el 
CAPÍTULO 7. IMPLEMENTACIÓN Y PRUEBAS DE CAMPO DEL DIAGNOSTICADOR EN EL HC NO TRIPULADO.

Tabla 7.12: Resumen Número de Fallos de Variables en Misiones

\begin{tabular}{|c|c|c|c|c|c|}
\hline Variable & \#F Normal & \#F Vib Plato & \#FVIb Cola & \#F Mix & \#F Mix Nor \\
\hline \hline Temperatura $T$ & 0 & 0 & 0 & 0 & 0 \\
\hline Voltaje $V$ & 0 & 0 & 0 & 0 & 0 \\
\hline Flujo Combustible $F$ & 0 & 0 & 0 & 0 & 0 \\
\hline RPMs & 6 & 10 & 3 & 25 & 3 \\
\hline Vibraciones $G$ & 93 & 28 & 26 & 53 & 48 \\
\hline I de Servos $I s$ & 0 & 1 & 0 & 0 & 0 \\
\hline Roll/Pitch/Yaw $\varnothing$ & 0 & 0 & 0 & 0 & 0 \\
\hline Sub. Motor $P_{C 1}$ & 6 & 10 & 3 & 25 & 3 \\
\hline Sub. Rotor Ppal $P_{C 2}$ & 93 & 28 & 26 & 53 & 48 \\
\hline Sub. Rotor Cola $P_{C 3}$ & 0 & 1 & 0 & 0 & 0 \\
\hline
\end{tabular}

suelo. Por lo tanto, el operador del UAS puede observar que una variable medida cae en alarma (advertencia) y en algunos casos en fallo por cortos períodos de tiempo y se recupera a su condición normal.

\section{Resultados de Vuelos Reales}

A continuación se presenta un resumen del comportamiento de las variables en los vuelos de comportamiento normal y de fallo. Se puede apreciar como la Red de Petri Diagnosticadora implementada en LabVIEW, ha podido identificar no solo las condiciones de alarma y fallo, si no el numero de veces que cada variable ha caído en umbral de fallo, con ello, la RdPD identifica también el número de veces que ha caído en fallo cada subsistema (Motor, Rotor y Cola), el resumen de todos los vuelos realizados se puede observar en la tabla 7.12.

Vuelo Normal (Duración $756 \mathrm{Sg}$ ): Como la figura 7.35 muestra, aunque los umbrales de RPM y vibración superan intermitentemente su umbral de fallo durante el vuelo, ninguna alarma fue activada. Es decir, la RdP diagnosticadora encontró que las variables RPM y vibraciones superaron su umbral de funcionamiento normal en 6 y 93 veces, respectivamente. Para más detalles sobre el vuelo en condiciones óptimas consulte la tabla 5. Es un vuelo con la aeronave trabajando en optimas condiciones, Se busca conocer el comportamiento de la aeronave en los diferentes estados: relenti, despegue, en vuelo y aterrizaje. Se puede observar que en momentos de relenti, despegue y aterrizaje tenemos 
CAPÍTULO 7. IMPLEMENTACIÓN Y PRUEBAS DE CAMPO DEL DIAGNOSTICADOR EN EL HC NO TRIPULADO.

Tabla 7.13: Comportamiento del HC en Vuelo Normal

\begin{tabular}{|c|c|c|c|}
\hline Estado & Descripción & Comportamiento & Fuente \\
\hline \hline 1,5 & Reposo (Motor encendido en tierra) & Alarma/Fallo & Vibraciones/RPM \\
\hline 2,6 & Despegue & Alarma & RPM \\
\hline 3 & Vuelo (Flotando, Translación) & Alarma & Vibraciones \\
\hline 4 & Aterrizaje & Alarma & Vibraciones/RPM \\
\hline
\end{tabular}

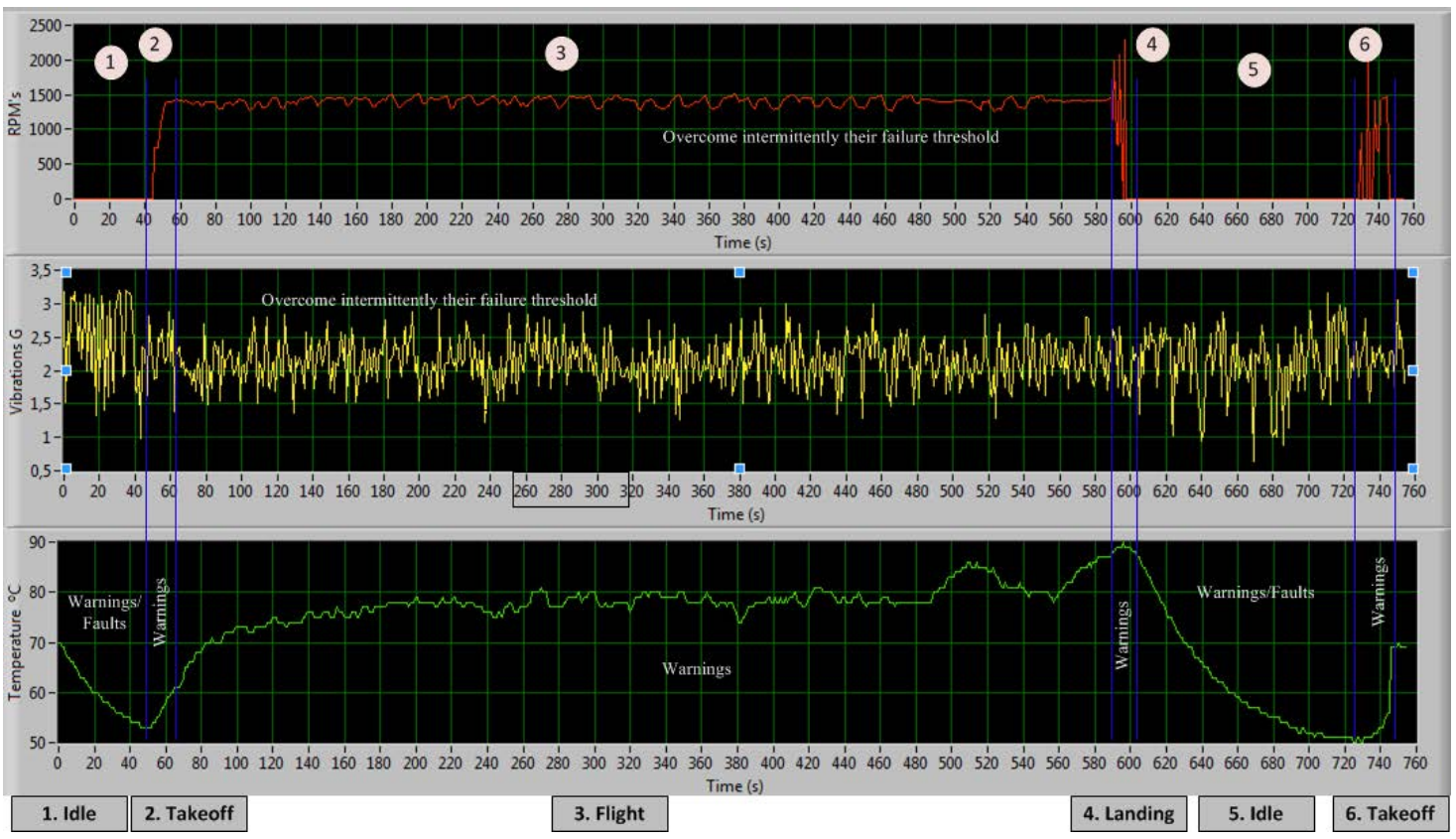

Figura 7.35: Vuelo Normal

que los umbrales de RPMS y Vibraciones caen en alarma, pero no se identifica un fallo en la aeronave. Para más detalles del vuelo ver la tabla 7.13.

Vuelo con Fallo de Vibraciones de Plato (Duración $181 \mathrm{Sg}$ ): Pequeñas tiras de cinta se añadieron a las palas del rotor principal para obligar a un desequilibrio de las palas, lo que resulta en una pérdida de sincronía cuando giran a alta velocidad. Por tanto, esto aumenta significativamente las vibraciones en la aeronave. Dado que el riesgo asumido era muy alto, solamente vuelos cortos se llevaron a cabo en estas circunstancias. De hecho, el vuelo realizado causó problemas en el silenciador (es decir, se afloja repetidamente y en un cierto momento el rotor de cola se perdió). Como muestra la figura 7.36, durante partes cortas de vuelo, la amplitud de vibración excedió su umbral y era imposible detectar un tiempo de comportamiento normal en la aeronave. Aunque la velocidad del rotor en vuelo es en condiciones normales, la temperatura 
CAPÍTULO 7. IMPLEMENTACIÓN Y PRUEBAS DE CAMPO DEL DIAGNOSTICADOR EN EL HC NO TRIPULADO.

Tabla 7.14: Comportamiento del HC en Vuelo con Fallo de Vibraciones de Plato

\begin{tabular}{|c|c|c|c|}
\hline Estado & Descripción & Comportamiento & Fuente \\
\hline \hline $1,3,9$ & Reposo (Motor encendido en tierra) & Alarma/Fallo & Vibrac./RPM \\
\hline $2,4,6$ & Despegue & Alarma/Fallo & Vibrac./RPM \\
\hline 5,7 & Vuelo (Flotando, Traslación) & Alarma/Fallo & Vibraciones \\
\hline 8 & Aterrizaje & Alarma & Vibrac./RPM \\
\hline
\end{tabular}

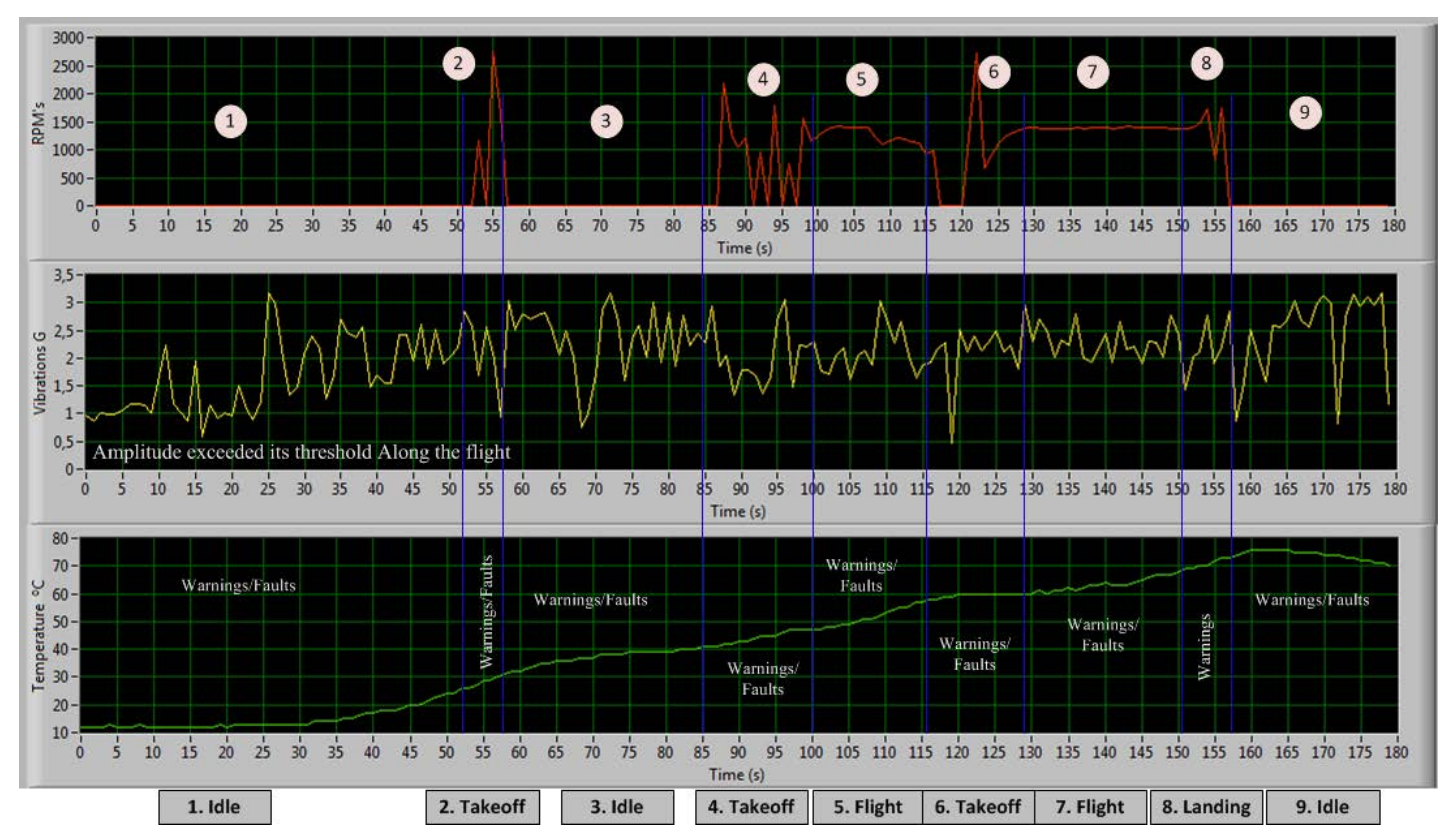

Figura 7.36: Vuelo Fallo Vibraciones Plato

aumenta rápidamente. Vibraciones cayeron en fallo 28 veces y el valor de RPM lo hicieron en 10 ocasiones. Más detalles sobre el vuelo en vibraciones placa de fallo se proporcionan en la tabla 7.14.

Vuelo con Fallo de Vibraciones de Cola (Duración $238 \mathrm{Sg}$ ): El procedimiento para crear las vibraciones fue similar al caso anterior, pero la cinta se aplicó a las palas del rotor de cola. El riesgo en este caso es incluso superior a la anterior, ya que las vibraciones en el rotor de cola se amplifican debido a la longitud de la cola. Por otra parte, las vibraciones afectan directamente al ámbito de la cola, creando problemas adicionales para la estabilización, por lo tanto, sólo vuelos cortos se llevaron a cabo. En esta misión, el piloto hizo grandes correcciones para elevar la aeronave. Como se muestra en la figura 7.37, las vibraciones están fuera de su umbral normal y la RdP diagnosticadora informa al piloto de un fallo de manera continua. La velocidad del rotor era bajo condicio- 
CAPÍTULO 7. IMPLEMENTACIÓN Y PRUEBAS DE CAMPO DEL DIAGNOSTICADOR EN EL HC NO TRIPULADO.

Tabla 7.15: Comportamiento del HC en Vuelo con Fallo de Vibraciones de Cola

\begin{tabular}{|c|c|c|c|}
\hline Estado & Descripción & Comportamiento & Fuente \\
\hline \hline 1 & Reposo (Motor encendido en tierra) & Alarma/Fallo & Vibrac./RPM \\
\hline 2 & Despegue & Alarma/Fallo & Vibrac./RPM \\
\hline 3 & Vuelo (Flotando, Translación) & Alarma/Fallo & Vibraciones \\
\hline 4 & Aterrizaje & Alarma & Vibrac./RPM \\
\hline 5 & Apagado & Sin señales & \\
\hline
\end{tabular}

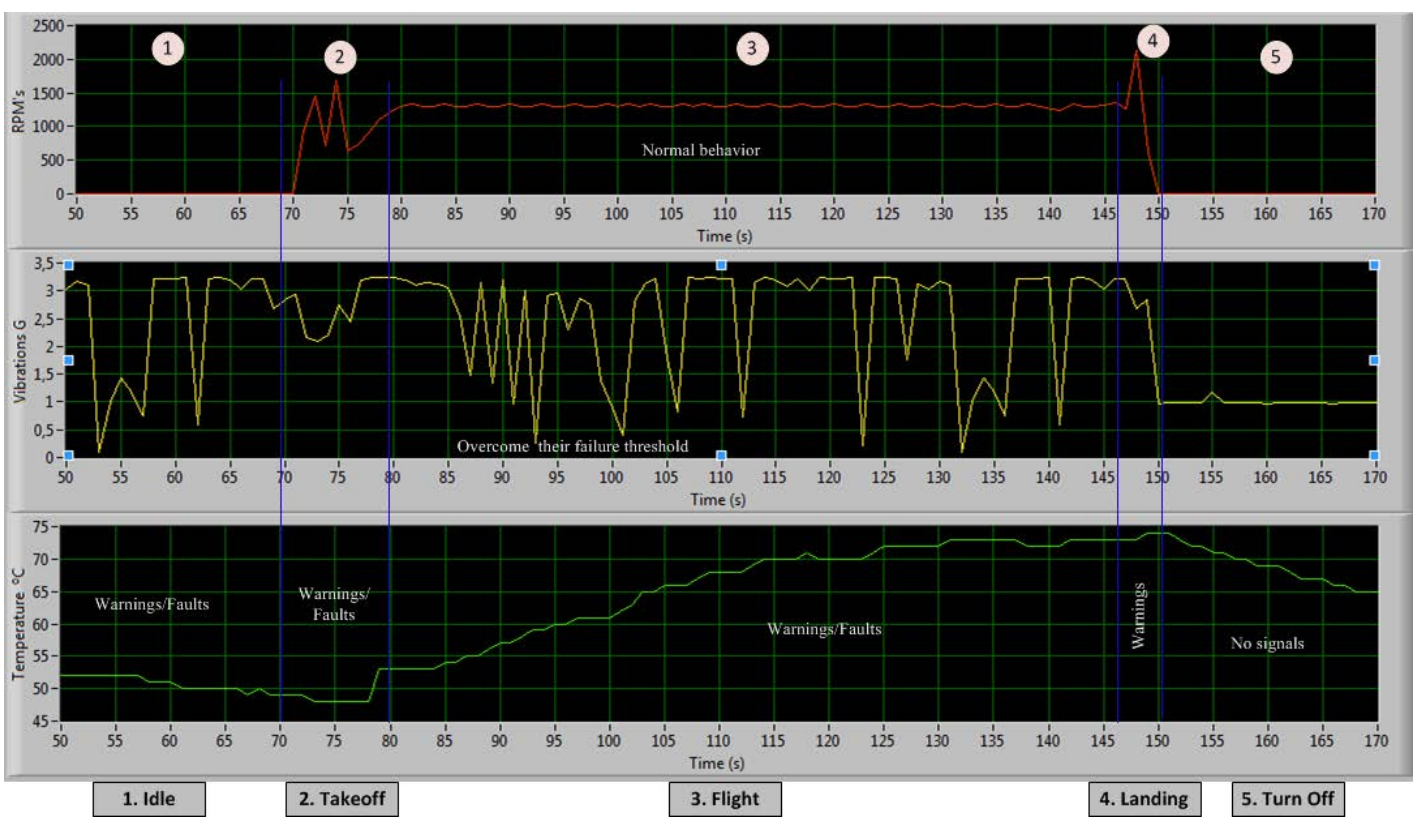

Figura 7.37: Vuelo Fallo Vibraciones de Cola

nes normal, aunque se obtuvo un cambio brusco en el tiempo requerido para el despegue y el aterrizaje. La RdPD detectó que los subsistemas del motor fallaron 3 veces y el subsistema de rotor principal fallaron 26 veces. La variable más crítica fue las vibraciones con 26 caídas, seguidas de la temperatura del motor que, aunque no cayó en fallo, sufrió un incremento en su pendiente debido a los esfuerzos exigido al motor por los comandos bruscos del piloto. La tabla 7.15 proporciona más detalles sobre el vuelo.

Vuelo con Fallo de Mezcla de Combustible (Duración $657 \mathrm{Sg}$ ). Este fallo fue generado por el cierre del colector de admisión. Esto afecta a la entrada del combustible en el carburador del helicóptero. Durante la primera parte del vuelo, el piloto tuvo que forzar el motor para obtener revoluciones suficiente para realizar el vuelo estacionario, en consecuencia, el silenciador salió de su lugar, y el 
CAPÍTULO 7. IMPLEMENTACIÓN Y PRUEBAS DE CAMPO DEL DIAGNOSTICADOR EN EL HC NO TRIPULADO.

Tabla 7.16: Comportamiento del HC en Vuelo con Fallo de Vibraciones de Cola

\begin{tabular}{|c|c|c|c|}
\hline Estado & Descripción & Comportamiento & Fuente \\
\hline \hline 1 & Reposo (Motor encendido en tierra) & Alarma/Fallo & Vibrac./RPM \\
\hline 2,6 & Despegue & Alarma & RPM \\
\hline 3 & Vuelo (Flotando, Translación) & Alarma/Fallo & Vibrac./RPM \\
\hline 4,8 & Aterrizaje & Alarma & Vibrac./RPM \\
\hline 5 & Apagado & Sin señales & \\
\hline 7 & Vuelo (Flotando, Translación) & Alarma/Fallo & Vibraciones \\
\hline
\end{tabular}

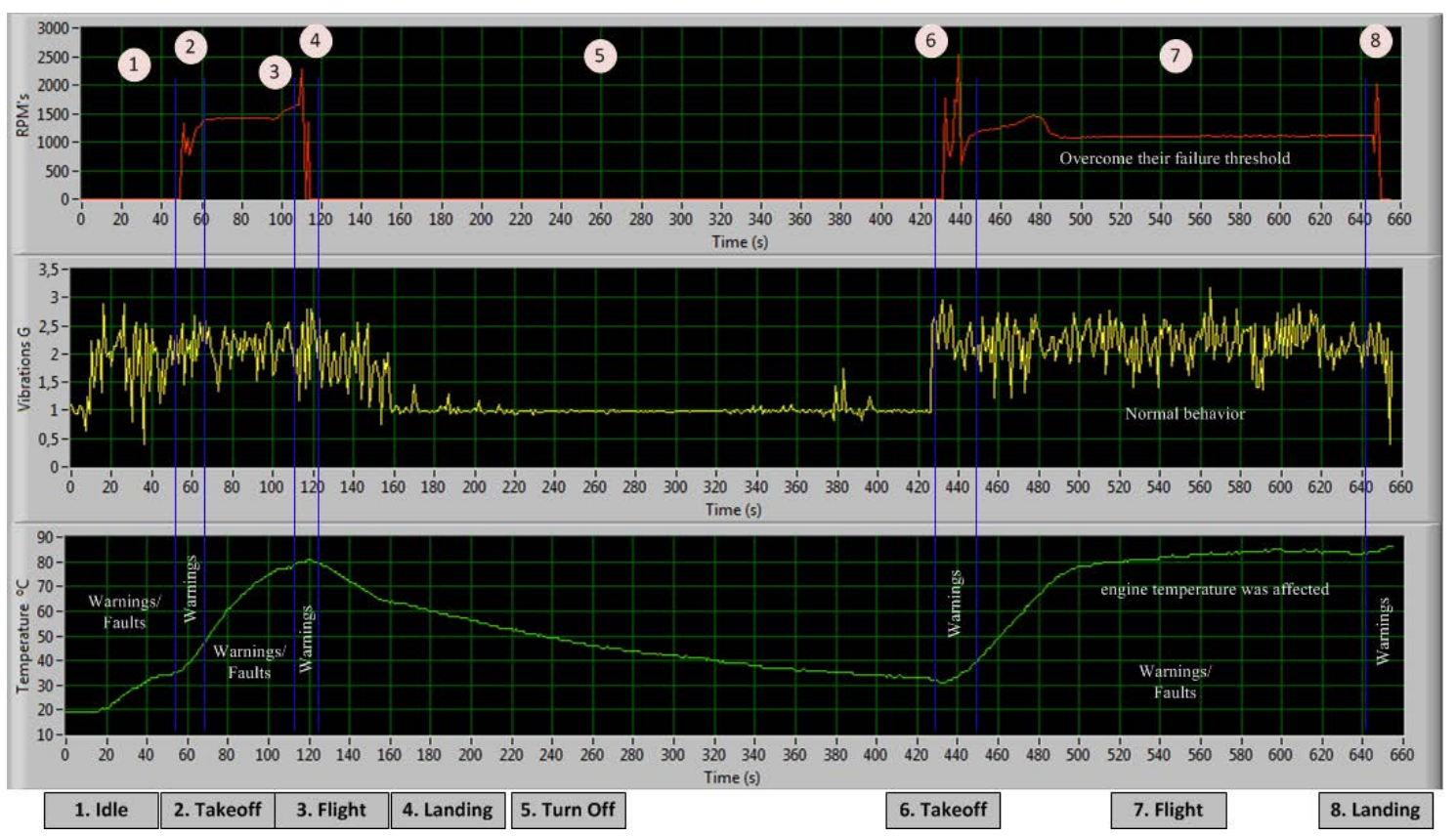

Figura 7.38: Vuelo Fallo de Mezcla

HC tuvo que ser vuelto a montar y re-iniciado el vuelo. Durante este vuelo, la velocidad del rotor salió de su umbral normal y las vibraciones se levantó con más frecuencia que la del comportamiento normal. En consecuencia, la temperatura del motor también se vio afectada y su pendiente aumentó como la figura 7.38 ilustra. La RdP diagnosticadora detectó fallos en el subsistema motor 25 veces, el subsistema del rotor principal falló 53 veces. Las variables más importantes fueron la velocidad del rotor y las vibraciones, seguido por la temperatura que, a pesar de no caer en fallo, su pendiente se incrementó por el alto número de maniobras necesarias para realizar el vuelo. Más detalles sobre el vuelo en la mezcla de combustible se pueden encontrar en la tabla 7.16. 
CAPÍTULO 7. IMPLEMENTACIÓN Y PRUEBAS DE CAMPO DEL DIAGNOSTICADOR EN EL HC NO TRIPULADO.

Tabla 7.17: Zonas de Trabajo para RPMs, Vibraciones, Temperatura

\begin{tabular}{|c|c|c|c|}
\hline & RPMs & Temperatura & Vibraciones \\
\hline \hline Normal & $1200-1400$ & $0-90$ & $0.6-2.5$ \\
\hline Advertencia & $(1100-1200) \circ(1400-1500)$ & $(90-100)$ & $(0.3-0.6) \circ(2-2.5)$ \\
\hline Fallo & $(0-1100) \circ(>1500)$ & $(>100)$ & $(0-0.3) \circ(>2.5)$ \\
\hline
\end{tabular}

Vuelo Normal Mix (Duración 714 Sg). De nuevo se pone la aeronave a punto, se intenta realizar el mismo vuelo anterior en estado normal para poder comparar el comportamiento de las variables. Se observó que las variables criticas (RPMs, vibraciones y temperatura) retornaron a su comportamiento normal, el piloto realiza un vuelo sin esfuerzos y el comportamiento del helicóptero es satisfactorio. La RdPD detecto que los subsistemas del motor falla 3 veces, que el subsistema del Plato falla 48 veces y que el subsistema de cola falla 0 veces, la variable críticas desaparecen pero se detecta que las vibraciones presenta 48 caídas en fallo.

\section{Zonas de trabajo.}

En la figura 7.39 se pueden apreciar las zonas de trabajo respecto a los niveles de vibración, RPM y temperatura del helicóptero. Las cuales, como se mencionó con anterioridad son las más relevantes a la hora de realizar la detección de fallos. Durante la experimentación y con la ayuda de pilotos expertos se establecieron las siguientes zonas de trabajo para las variables de RPM, temperatura y vibración, ver tabla 7.17.

En la gráfica se pueden observar los valores normales de funcionamiento (azul), alarma (amarillo) y fallo (rojo) dependiendo del estado de las variables de estudio y los rangos de funcionamiento establecidos anteriormente. Por otro lado, se observa que cuando dos niveles de advertencia se obtienen en el mismo momento, para algún par de estas variables tan importantes, se considera que existe una situación fuera de lo normal y se considera como fallo, informando al piloto la necesidad de bajar el helicóptero en vuelo o de realizar una comprobación del estado de tratarse de un análisis posterior. En general estas zonas son utilizadas por la red de Petri en conjunto con el tiempo de permanencia en las mismas y el número de ocurrencias para indicar el estado del helicóptero y realizar las indicaciones pertinentes. 
a) Zonas de trabajo: Vibración Vs Temepratura

b) Zonas de trabajo: RPM Vs Temperatura
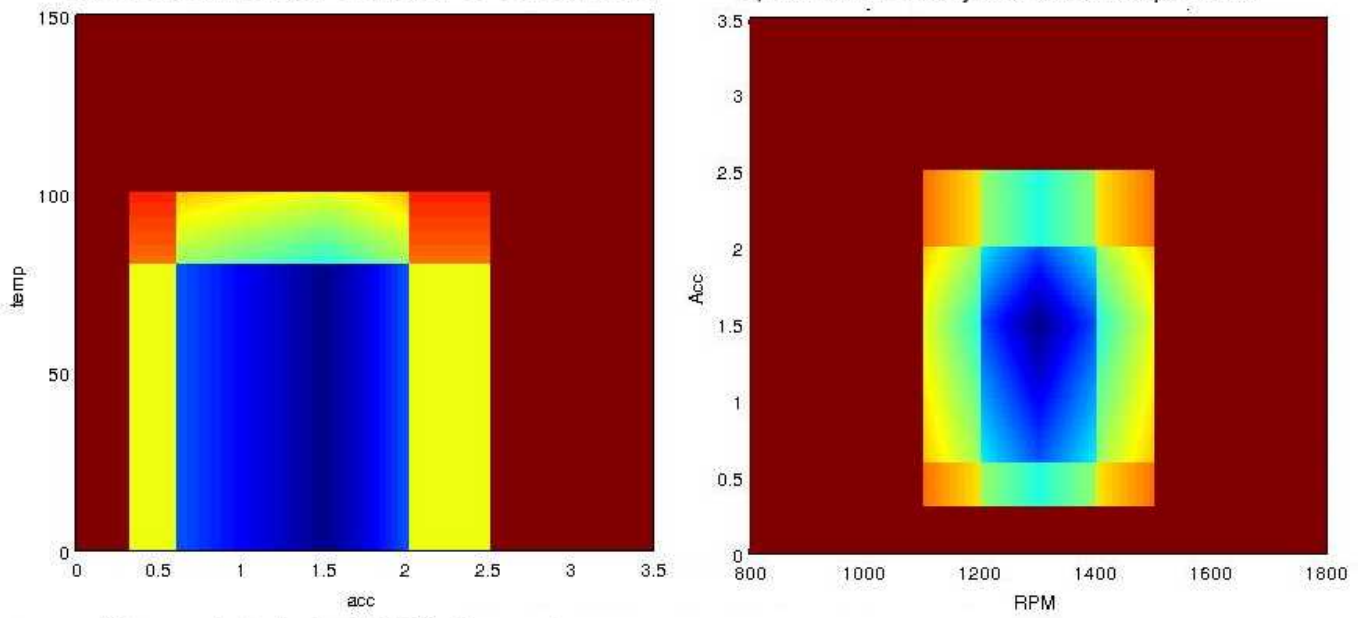

c) Zonas de trabajo: RPM Vs Temperatura
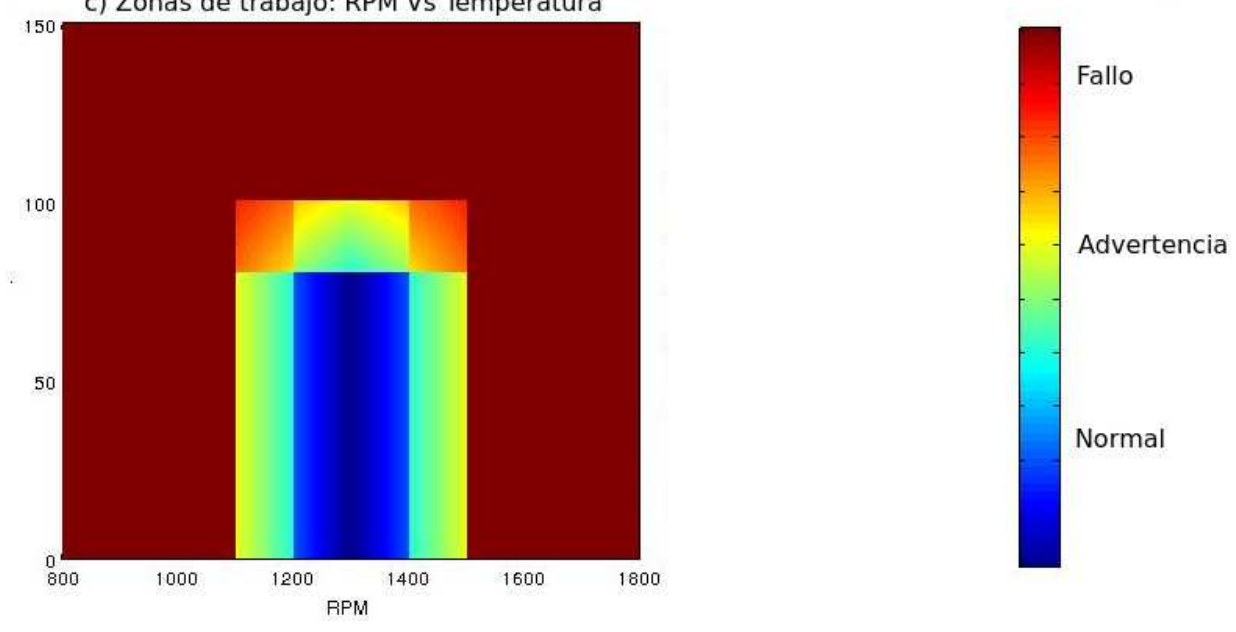

Figura 7.39: Zonas de Trabajo Normal, Advertencia y Fallo de RPMS, Vibraciones y Temperatura 
CAPÍTULO 7. IMPLEMENTACIÓN Y PRUEBAS DE CAMPO DEL DIAGNOSTICADOR EN EL HC NO TRIPULADO. 


\section{Capítulo 8}

\section{Conclusión}

En este trabajo de investigación se ha abordado el tema de diagnóstico de fallos mediante Redes de Petri. El documento inicia con un resumen de los algoritmos de diagnóstico de fallos de sistemas continuos, se presentaron algunas ventajas y desventajas en la clasificación de los algoritmos. La clasificación es hecha basada en modelos cualitativos, cuantitativos y basados en historia del proceso, esta clasificación es una buena orientación para conocer el estado del arte de los algoritmos de FDI que se han desarrollado en los últimos años.

Fundamental en cualquier investigación es conocer los desarrollos alternos que se están realizando, para el caso particular del diagnóstico de fallos de SED's, sistemas híbridos y su aplicación en el campo de las aeronaves no tripuladas han sido presentados, se construye un buen balance de las últimas investigaciones hechas mediante las MEF's y las RdP. Quedó definido cuales son las falencias de estos trabajos y qué se debía mejorar como proyecto de investigación, además, esto permitió definir unas características fundamentales a tener en cuenta cuando se desarrollen algoritmos de diagnóstico de fallos en SED's.

Se presentaron conceptos básicos de RdP necesarios para el proceso de construcción sistemático del diagnosticador. Esta herramienta de diagnóstico de fallos incluye: construcción del modelo que describe el comportamiento normal (transiciones observables) y de fallo (transiciones no observables). Posteriormente se construyó un modelo general de integración, el cual es refinado para que sólo esté compuesto por transiciones observables. Basado en el modelo se diseña y construye el diagnosticador. Para corroborar que la técnica 
tiene un buen desempeño se construyen dos ejemplos de aplicación, primero en un sistema de aire acondicionado y calefacción (HVAC) y después es aplicado al sistema del proceso de envasado, los resultados son óptimos ya que la metodología es muy fácil de implementar. Se incluyó el FDI en SED's con Fl's, incluyendo la dinámica temporal. Basado en el mismo ejemplo del proceso de envasado, proceso híbrido, se realiza un FDI basado en RdP Híbridas que permite una extensión del trabajo realizado solo para SED's.

La parte principal de esta investigación fue la de abordar el diagnóstico de fallos aplicado a un UAS mediante el uso de redes de Petri. Un proceso metodológico para crear el modelo y el diagnosticador se ha presentado. El proceso se ha dividido en subsistemas. Esto permite evaluar los fallos de una manera independiente. Como resultado más destacado, la red de petri diagnosticadora creada es capaz de detectar no sólo las fallos permanentes sino también intermitentes, ofreciendo al piloto un conjunto de alarmas de vigilancia de la salud de la aeronave. Esto puede ser considerado como el principal aporte de este trabajo, ya que ningún trabajo previo en la literatura muestra el uso de este tipo de diagnosticador basado en redes de Petri. Otras ventajas del método propuesto son la capacidad de ser implementado en procesos complejos, la flexibilidad para hacer cambios (es decir, incluir dispositivos adicionales en el diagnosticador), la reducción de la explosión combinacional, y una construcción sistemática.

Una aplicación en un sistema real (un helicóptero no tripulado) ha sido presentado como demostración. Una sección especial dedicada a analizar los diferentes vuelos realizados para validar el sistema de diagnosis forzando algunos mal funcionamiento en la aeronave se ha incluido en este trabajo. Hay que subrayar que obligar a varios fallos durante los vuelos resultó ser muy arriesgado y por lo tanto difícil de repetir intensamente. Un análisis a fondo que justifica las variables seleccionadas para ser monitoreadas y sus conexiones con las causas más frecuentes de fallo en los helicópteros se ha incluido también. Dado que el helicóptero es una plataforma muy vulnerable, se requiere un método de diagnóstico de fallos robusto. Aunque los experimentos reales suponen un alto riesgo, una cantidad valiosa de vuelos se han realizado con el fin de evaluar el rendimiento de la herramienta y de la metodología. Además, el sistema de adquisición de datos diseñado y construido para esta aplicación es descrito. Por otra parte, el uso de una herramienta de uso común, tal como LabVIEW permite 
proporcionar al diagnosticador las características adicionales que resultan de gran importancia para el mantenimiento preventivo.

Finalmente, se presentó el desarrollo de la instrumentación necesaria para la captura y visualización de las variables criticas, que componen la aeronave no tripulada (helicóptero); se construye una plataforma con comunicación inhalámbrica, paralela e independiente a los sistemas de control de la aeronave. La información del comportamiento de dichas variables es almacenada por características de redundancia, tanto en la aeronave como en la estación de control en tierra. Por primera vez, se recopila la experiencia de pilotos y grupos de investigación, para establecer puntos críticos en las aeronaves de radio control. La electrónica diseñada y embarcada en la aeronave, cumple con las limitaciones de peso y dimensiones típicas de las UAS. Aunque este proyecto hace parte de una investigación de diagnóstico de fallos de sistemas híbridos, modelado con Redes de Petri, la información recogida con esta herramienta, podrá usarse como trabajo para validar otras técnicas de diagnóstico de fallos y construir modelos matemáticos de este tipo de aeronaves, en donde se implementen estrategias basadas en Lógica Fuzzy, Redes Neuronales, Control Inteligente, entre otros. También sería fundamental realizar un análisis espectral de la vibración, con el objetivo de encontrar más tipos de daños mecánicos, la generación de modelos matemáticos de la operación normal y fallo de la aeronave. 


\section{Bibliografía}

[1] Modelica, 2015.

[2] Rajeev Alur and Joel M. Esposito. CHARON, Modular Specification of Hybrid Systems, 2015.

[3] Muhammad Asim, DR Nadeem Ehsan, and Khalid Rafique. Probable Causal Factors In UAV Accidents Based on Human Factor Análysis and Classification System. In 27TH INTERNATIONAL CONGRESS OF THE AERONAUTICAL SCIENCES, Nice, France, September 2010.

[4] Antonio Barrientos, Pedro Gutierrez, and Julian Colorado. Advanced UAV Trajectory Generation: Planning and Guidance. In Thanh Mung, editor, Aerial Vehicles. InTech, January 2009.

[5] Michèle Basseville. Detecting changes in signals and systems-A survey. Automatica, 24(3):309-326, May 1988.

[6] Michèle Basseville, Igor V. Nikiforov, and others. Detection of abrupt changes: theory and application, volume 104. Prentice Hall Englewood Cliffs, 1993.

[7] F. Bateman, H. Noura, and M. Ouladsine. Actuators Fault Diagnosis and Tolerant Control for an Unmanned Aerial Vehicle. In IEEE International Conference on Control Applications, 2007. CCA 2007, pages 1061-1066, October 2007.

[8] Francois Bateman, Hassan Noura, and Mustapha Ouladsine. Active fault detection and isolation strategy for an unmanned aerial vehicle with redundant flight control surfaces. pages 1246-1251. IEEE, June 2008.

[9] California PATH UC Berkeley. SHIFT, June 1998. 
[10] M. Bonfè, P. Castaldi, W. Geri, and S. Simani. Fault detection and isolation for on-board sensors of a general aviation aircraft. International Journal of Adaptive Control and Signal Processing, 20(8):381-408, October 2006.

[11] Maria Paola Cabasino, Alessandro Giua, and Carla Seatzu. Fault detection for discrete event systems using Petri nets with unobservable transitions. Automatica, 46(9):1531-1539, September 2010.

[12] Guowei Cai, Ben M. Chen, Kemao Peng, Miaobo Dong, and Tong Heng Lee. Modeling and control of the yaw channel of a UAV helicopter. Industrial Electronics, IEEE Transactions on, 55(9):3426-3434, 2008.

[13] Gail Carpenter, Stephen Grossberg, and others. The ART of adaptive pattern recognition by a self-organizing neural network. Computer, 21(3):7788, 1988.

[14] Christos G. Cassandras. From discrete event to hybrid systems. In Discrete Event Systems, 2002. Proceedings. Sixth International Workshop on, pages 3-8. IEEE, 2002.

[15] Sheug-Luen Chung, Chien-Chung Wu, and MuDer Jeng. Failure diagnosis: a case study on modeling and analysis by Petri nets. In Systems, Man and Cybernetics, 2003. IEEE International Conference on, volume 3, pages 2727-2732. IEEE, 2003.

[16] O. Contant, S. Lafortune, and D. Teneketzis. Failure diagnosis of discrete event systems: the case of intermittent faults. In Proceedings of the 41st IEEE Conference on Decision and Control, 2002, volume 4, pages 40064011 vol.4, December 2002.

[17] Lennon R. Cork, Rodney A. Walker, and Shane Dunn. Fault Detection, Identification and Accommodation Techniques for Unmanned Airborne Vehicles. Australian International Aerospace Congress (AIAC), 2005.

[18] Antonio Correcher, Emilio García, Francisco Morant, Eduardo Quiles, and Leonardo Rodríguez. Intermittent failure dynamics characterization. Reliability, IEEE Transactions on, 61(3):649-658, 2012.

[19] George Cybenko. Approximation by superpositions of a sigmoidal function. Mathematics of control, signals and systems, 2(4):303-314, 1989. 
[20] René David and Hassane Alla. Discrete, continuous, and hybrid Petri nets, volume 1. Springer, 2005.

[21] Conrado Daws, Alfredo Olivero, Stavros Tripakis, and SergioYovine. Kronos, 2002.

[22] Johan De Kleer and John Seely Brown. A qualitative physics based on confluences. Artificial Intelligence, 24(1):7-83, December 1984.

[23] UC Berkeley EECS Dept. Ptolemy II, December 2014.

[24] Graham R. Drozeski, Bhaskar Saha, and George J. Vachtsevanos. A fault detection and reconfigurable control architecture for unmanned aerial vehicles. In Aerospace Conference, 2005 IEEE, pages 1-9. IEEE, 2005.

[25] Michael Elgersma and Sonja Glavaski. Reconfigurable control for active management of aircraft system failures. In American Control Conference, 2001. Proceedings of the 2001, volume 4, pages 2627-2639. IEEE, 2001.

[26] Eurocontrol. National RPAS Regulations, 2015.

[27] Sriram Narasimhan Feng, Feng Zhao, Gautam Biswas, and Elmer Hung. Fault isolation in hybrid systems combining model based diagnosis and signal processing. In In Proc. 4th IFAC Symp. SAFEPROCESS. Citeseer, 2000.

[28] Francis Eric Finch and Mark A. Kramer. Narrowing diagnostic focus using functional decomposition. A/ChE journal, 34(1):25-36, 1988.

[29] G. K. Fourlas, K. J. Kyriakopoulos, and N. J. Krikelis. Fault diagnosis of hybrid systems. In Intelligent Control, 2005. Proceedings of the 2005 IEEE International Symposium on, Mediterrean Conference on Control and Automation, pages 832-837. IEEE, 2005.

[30] PAUL M. FRANK. On-line fault detection in uncertain nonlinear systems using diagnostic observers: a survey. International Journal of Systems Science, 25(12):2129-2154, December 1994. 
[31] PM Frank and J W\{ü\}nnenberg. Robust fault diagnosis using unknown input schemes. In RJ Patton, PM Frank, and RN Clark, editors, Fault Diagnosis in Dynamic Systems: Theory and Application, pages 47-98. Prentice Hall, 1989.

[32] Paul Michael Freeman. Reliability Assessment for Low-cost Unmanned Aerial Vehicles. PhD thesis, UNIVERSITY OF MINNESOTA, 2014.

[33] E. García, A. Correcher, F. Morant, E. Quiles, and R. Blasco. Modular Fault Diagnosis Based on Discrete Event Systems. Discrete Event Dynamic Systems, 15(3):237-256, August 2005.

[34] R. D. Garcia, K. P. Valavanis, and A. Kandel. Autonomous helicopter navigation during a tail rotor failure utilizing fuzzy logic. In Control \& Automation, 2007. MED'07. Mediterranean Conference on, pages 1-6. IEEE, 2007.

[35] S. Genc and S. Lafortune. Distributed Diagnosis of Place-Bordered Petri Nets. IEEE Transactions on Automation Science and Engineering, 4(2):206-219, April 2007.

[36] J. Gertler, Xiaowen Fang, and Qiang Luo. Detection and diagnosis of plant failures: the orthogonal parity equation approach. Control and Dynamic Systems, 37:159, 2012.

[37] Janos J. Gertler and Ramin Monajemy. Generating directional residuals with dynamic parity relations. Automatica, 31(4):627-635, 1995.

[38] Victor Girondin, Hervé Morel, Jean Philippe Cassar, and Komi Midzodzi Pekpe. Vibration-based fault detection of sharp bearing faults in helicopters. In SAFEPROCESS, pages 180-185, 2012.

[39] Sonja Glavaski and Michael Elgersma. Active aircraft fault detection and isolation. In AUTOTESTCON Proceedings, 2001. IEEE Systems Readiness Technology Conference, pages 692-705. IEEE, 2001.

[40] Kelly J. Hayhurst, Jeffrey M. Maddalon, Paul S. Miner, Michael P. DeWalt, and G. Frank McCormick. Unmanned aircraft hazards and their implications for regulation. In 25th Digital Avionics Systems Conference, 2006 IEEE/AIAA, pages 1-12. IEEE, 2006. 
[41] Thomas A. Henzinger. The Theory of Hybrid Automata. In M. Kemal Inan and Robert P. Kurshan, editors, Verification of Digital and Hybrid Systems, number 170 in NATO ASI Series, pages 265-292. Springer Berlin Heidelberg, 2000. DOI: 10.1007/978-3-642-59615-5_13.

[42] Tom Henzinger, Pei-Hsin Ho, and Howard Wong-Toi. HyTech: The HYbrid TECHnology Tool, 2015.

[43] G. Heredia and A. Ollero. Sensor fault detection in small autonomous helicopters using observer/Kalman filter identification. In IEEE International Conference on Mechatronics, 2009. ICM 2009, pages 1-6, April 2009.

[44] G. Heredia, A. Ollero, M. Bejar, and R. Mahtani. Sensor and actuator fault detection in small autonomous helicopters. Mechatronics, 18(2):90-99, 2008.

[45] Guillermo Heredia, Aníbal Ollero, Rajesh Mahtani, Manuel Béjar, Volker Remu $\backslash$ s s, and Marek Musial. Detection of sensor faults in autonomous helicopters. In IEEE International Conference on Robotics and Automation, volume 2, page 2229. IEEE; 1999, 2005.

[46] Guillermo Heredia and Anibal Ollero. Detection of sensor faults in small helicopter UAVs using observer/Kalman filter identification. Mathematical Problems in Engineering, 2011, 2011.

[47] Hongge Hu, Dagui Huang, Hong Hu, and Guangying Sun. Combining modeling and fault detection in automated manufacturing systems based on hybrid Petri net. In Proc. IEEE International Conference on Intelligent Mechatronics and Automation, pages 728-732, 2004.

[48] Ronan HUBERT. Bureau of Aircraft Accidents Archives, 2015.

[49] R Isermann. Process Fault Diagnosis Based on Dynamic Models and Parameter EstimationMethods. In R Patton, PM Frank, and R Clark, editors, Fault Diagnosis in Dynamic Systems - Theory and Applications. Prentice Hall International, 1989.

[50] Yumi Iwasaki and Herbert A. Simon. Causality in device behavior. Artificial Intelligence, 29(1):3-32, July 1986. 
[51] J. Edward Jackson. A User's Guide to Principal Components. John Wiley \& Sons, January 2005.

[52] Shengbing Jiang, R. Kumar, and H.E. Garcia. Diagnosis of repeated/intermittent failures in discrete event systems. IEEE Transactions on Robotics and Automation, 19(2):310-323, April 2003.

[53] Gertler JJ. Analytical Redundancy Methods in Fault Detection and Isolation. IFAC Fault Detection, Supervision and Safety for Tech. Process., 6, 1992.

[54] Kim Larsen Johan Bengtsson. UPPAAL - a Tool Suite for Automatic Verification of Real-Time Systems. 1996.

[55] V.K. Kaliappan, H. Young, A. Budiyono, and Dugki Min. Fault tolerant controller design for component faults of a small scale unmanned aerial vehicle. In 2011 8th International Conference on Ubiquitous Robots and Ambient Intelligence (URAI), pages 79-84, November 2011.

[56] Herbert Kay and Benjamin Kuipers. Numerical Behavior Envelopes for Qualitative Models. In AAAl, pages 606-613, 1993.

[57] B. Köppen-Seliger, P. M. Frank, and A. Wolff. Residual evaluation for fault detection and isolation with RCE neural networks. In American Control Conference, Proceedings of the 1995, volume 5, pages 3264-3268. IEEE, 1995.

[58] Bruce H. Krogh. Recent advances in discrete analysis and control of hybrid systems. In Discrete Event Systems, 2002. Proceedings. Sixth International Workshop on, pages 311-314. IEEE, 2002.

[59] W.S. Lee, D.L. Grosh, F.A. Tillman, and C.H. Lie. Fault Tree Analysis, Methods, and Applications \#2013; A Review. IEEE Transactions on Reliability, R-34(3):194-203, August 1985.

[60] F. P. Lees. Fault Detection ond Diagnosis in Chemical and Petrochemicol Processes, by D. M. Himmelblau, Scientific Publishing Co., 1978, 414 pages, \$59.50. AlChE Journal, 25(5):907-907, September 1979. 
[61] Ruoyu Li, Serap Ulusam Seçkiner, David He, Eric Bechhoefer, and Praneet Menon. Gear fault location detection for split torque gearbox using AE sensors. Systems, Man, and Cybernetics, Part C: Applications and Reviews, IEEE Transactions on, 42(6):1308-1317, 2012.

[62] Lijun Liu, Yi Shen, and Earl H. Dowell. Integrated adaptive fault-tolerant $\mathrm{h}$ infinity output feedback control with adaptive fault identification. Journal of Guidance, Control, and Dynamics, 35(3):881-889, 2012.

[63] A. Mancini, F. Caponetti, E. Frontoni, P. Zingaretti, S. Longhi, and others. Safe flying for an UAV Helicopter. In Control \& Automation, 2007. MED'07. Mediterranean Conference on, pages 1-6. IEEE, 2007.

[64] Sheila Mcllraith, Gautam Biswas, Dan Clancy, and Vineet Gupta. Hybrid systems diagnosis. In Hybrid Systems: Computation and Control, pages 282-295. Springer, 2000.

[65] Robert Milne. Strategies for diagnosis. IEEE Transactions on Systems, Man, \& Cybernetics, 1987.

[66] Tadao Murata. Petri nets: Properties, analysis and applications. Proceedings of the IEEE, 77(4):541-580, 1989.

[67] Saul Montesde Oca, Vicenc Puig, Marcin Witczak, and Joseba Quevedo. Fault-tolerant control of a two-degree of freedom helicopter using LPV techniques. In Control and Automation, 2008 16th Mediterranean Conference on, pages 1204-1209. IEEE, 2008.

[68] Office of the Secretary of Defense. Unmanned Aerial Vehicle Reliability Study. Technical report, February 2003.

[69] Vicenç Puig, Joseba Quevedo, Teresa Escobet, Bernardo Morcego, and Carlos Ocampo. Control tolerante a fallos (parte I): fundamentos y diagnóstico de fallos. Revista lberoamericana de automática e informática industrial, 1(1):15-31, 2004.

[70] J. T. Qi and J. D. Han. Adaptive UKF and its application in fault tolerant control of rotorcraft unmanned aerial vehicle. Journal of Mechanical Engineering, 45(4):115-125, 2009. 
[71] Jun-tong Qi and others. Application of wavelets transform to fault detection in rotorcraft UAV sensor failure. Journal of Bionic Engineering, 4(4):265270, 2007.

[72] Juntong Qi and Jianda Han. Fault adaptive control for RUAV actuator failure with unscented Kalman filter. In Innovative Computing Information and Control, 2008. ICICIC'08. 3rd International Conference on, pages 169169. IEEE, 2008.

[73] Juntong Qi, Jianda Han, and Zhenwei Wu. Rotorcraft UAV actuator failure estimation with KF-based adaptive UKF algorithm. In American Control Conference, 2008, pages 1618-1623. IEEE, 2008.

[74] Juntong Qi, Zhe Jiang, Xingang Zhao, and Jianda Han. Fault detection design for RUAV with an adaptive threshold neural-network scheme. In Control and Automation, 2007. ICCA 2007. IEEE International Conference on, pages 554-559. IEEE, 2007.

[75] Juntong Qi, Dalei Song, Chong Wu, Jianda Han, and Tianran Wang. KFbased adaptive UKF algorithm and its application for rotorcraft UAV actuator failure estimation. Int J Adv Robotic Sy, 9(132), 2012.

[76] Juntong Qi, Xingang Zhao, Zhe Jiang, and Jianda Han. An adaptive threshold neural-network scheme for rotorcraft UAV sensor failure diagnosis. In Advances in Neural Networks-ISNN 2007, pages 589-596. Springer, 2007.

[77] Peter J. Ramadge and W. Murray Wonham. Supervisory control of a class of discrete event processes. SIAM journal on control and optimization, 25(1):206-230, 1987.

[78] Antonio Ramírez-Treviño, Elvia Ruiz-Beltrán, Israel Rivera-Rangel, and Ernesto López-Mellado. Online fault diagnosis of discrete event systems. A Petri net-based approach. Automation Science and Engineering, IEEE Transactions on, 4(1):31-39, 2007.

[79] Jens Rasmussen. The role of hierarchical knowledge representation in decisionmaking and system management. Systems, Man and Cybernetics, IEEE Transactions on, (2):234-243, 1985. 
[80] Raghunathan Rengaswamy and Venkat Venkatasubramanian. A fast training neural network and its updation for incipient fault detection and diagnosis. Computers \& Chemical Engineering, 24(2):431-437, 2000.

[81] M. Rezai, P. D. Lawrence, and M. R. Ito. Analysis of faults in hybrid systems by global Petri nets. In Systems, Man and Cybernetics, 1995. InteIligent Systems for the 21st Century., IEEE International Conference on, volume 3, pages 2251-2256. IEEE, 1995.

[82] Steven H. Rich and V. Venkatasubramanian. Model-based reasoning in diagnostic expert systems for chemical process plants. Computers \& Chemical Engineering, 11(2):111-122, 1987.

[83] Diego Ruiz. Fault diagnosis in chemical plants integrated to the information system. TDX (Tesis Doctorals en Xarxa), June 2001.

[84] Elvia Ruiz-Beltrán, E. López-Mellado, M. Meda-Campaña, and others. Fault detection and location in DES using Petri nets. In Systems, Man and Cybernetics, 2005 IEEE International Conference on, volume 2, pages 1645-1650. IEEE, 2005.

[85] Sikandar Samar, Dimitry Gorinevsky, and Stephen P. Boyd. Embedded estimation of fault parameters in an unmanned aerial vehicle. In Computer Aided Control System Design, 2006 IEEE International Conference on Control Applications, 2006 IEEE International Symposium on Intelligent Control, 2006 IEEE, pages 3265-3270. IEEE, 2006.

[86] Meera Sampath. A discrete event systems approach to failure diagnosis. PhD thesis, Wayne State University, 1995.

[87] Meera Sampath, Raja Sengupta, StCphane Lafortune, Kasim Sinnamohideen, and Demosthenis Teneketzis. Diagnosability of discrete-event systems. Automatic Control, IEEE Transactions on, 40(9):1555-1575, 1995.

[88] Meera Sampath, Raja Sengupta, Stephane Lafortune, Kasim Sinnamohideen, and Demosthenis C. Teneketzis. Failure diagnosis using discreteevent models. Control Systems Technology, IEEE Transactions on, 4(2):105-124, 1996. 
[89] David Siegel, Canh Ly, and Jay Lee. Methodology and framework for predicting helicopter rolling element bearing failure. Reliability, IEEE Transactions on, 61(4):846-857, 2012.

[90] Manuel Silva Suárez. Las redes de Petri: en la automática y la informática. Editorial AC, D.L. 1985, Madrid, 1985.

[91] Miguel Trigos, Antonio Barrientos, Jaime Del Cerro, and Hermes Lopez. Modelling and Fault Diagnosis by Means of Petri Nets. Unmanned Aerial Vehicle Application. In Pawel Pawlewski, editor, Petri Nets Applications. InTech, February 2010.

[92] Miguel A. Trigos, Antonio Barrientos, and Jaime del Cerro. Diagnóstico de Fallos de un UAV. DAQ para la Aplicación Real del Algoritmo. In Congreso Latinoamericano de Control Automático, CLCA 2012, Lima, Perú, 2012.

[93] Miguel A. Trigos, Antonio Barrientos, and Jaime del Cerro. Diagnosticador de Fallos Basado en Redes de Petri y LabVIEW para Evaluar el Estado de un UAV. In Congreso Latinoamericano de Control Automático, CLCA 2014, Cancún, Quintana Roo, México, October 2014.

[94] Miguel A. Trigos, Antonio Barrientos, and Jaime del Cerro. Systematic Process for Building a Fault Diagnoser Based on Petri Nets Applied to a Helicopter. Mathematical Problems in Engineering, 2015:e963756, October 2015 .

[95] Miguel A. Trigos, Emilio Garcia, and Leonardo Rodríguez. Modelado y Diagnóstico de Fallos por Medio de Redes De Petri de un Sistema de Envasado de Líquidos. In Congreso Latinoamericano de Control Automático, CLCA 2008, Merida, Venezuela, 2008.

[96] Miguel A. Trigos and Jair Leandro Landínez Salazar. Diagnóstico de Fallos de un UAV. DAQ para la Aplicación Real del Algoritmo. In Congreso Latinoamericano de Control Automático, CLCA 2010, Santiago, Chile, 2010.

[97] Miguel A. Trigos, Diego Mendez, Javier Garcia, Antonio Barrientos, and Jaime Del Cerro. Diseño e Implementación de la Instrumentación para la Lectura de Variables Críticas en Aeronaves no Tripuladas (UAS). In 
Congreso Latinoamericano de Control Automático, CLCA 2010, Santiago, Chile, 2010.

[98] M.A. Trigos Martinez and Emilio Garcia Moreno. Fault diagnosis and modeling of the liquids packaging process. A research based on Petri Nets. In 10th International Conference on Control, Automation, Robotics and Vision, 2008. ICARCV 2008, pages 1620-1624, December 2008.

[99] Miguel Angel Trigos Martínez and Jair Leandro Landínez Salazar. Sistema de envasado de líquidos modelado con redes de Petri y simulado con LabView y DSC. ITECKNE: Innovación e Investigación en Ingeniería, 8(2):147-155, 2011.

[100] Nancy H. Ulerich and Gary J. Powers. On-line hazard aversion and fault diagnosis in chemical processes: the digraph+ fault-tree method. Reliability, IEEE Transactions on, 37(2):171-177, 1988.

[101] Toshimitsu Ushio, Isao Onishi, and Koji Okuda. Fault detection based on Petri net models with faulty behaviors. In Systems, Man, and Cybernetics, 1998. 1998 IEEE International Conference on, volume 1, pages 113-118. IEEE, 1998.

[102] V. Venkatasubramanian and S. H. Rich. An object-oriented two-tier architecture for integrating compiled and deep-level knowledge for process diagnosis. Computers \& Chemical Engineering, 12(9):903-921, 1988.

[103] Venkat Venkatasubramanian, Raghunathan Rengaswamy, Kewen Yin, and Surya N. Kavuri. A review of process fault detection and diagnosis: Part I: Quantitative model-based methods. Computers \& Chemical Engineering, 27(3):293-311, March 2003.

[104] Arne Wahrburg and Jürgen Adamy. Robust fault isolation observers for non-square systems-a parametric approach. In Fault Detection, Supervision and Safety of Technical Processes, volume 8, pages 1275-1280, 2012.

[105] Ronaldo Waschburger, Henrique Mohallem Paiva, Roberto Kawakami Harrop Galv, and others. Fault detection in a laboratory helicopter employing 
a wavelet-based analytical redundancy approach. In Control and FaultTolerant Systems (SysTol), 2010 Conference on, pages 70-75. IEEE, 2010.

[106] Alan S. Willsky. A survey of design methods for failure detection in dynamic systems. Automatica, 12(6):601-611, 1976.

[107] Svante Wold, Kim Esbensen, and Paul Geladi. Proceedings of the Multivariate Statistical Workshop for Geologists and GeochemistsPrincipal component analysis. Chemometrics and Intelligent Laboratory Systems, 2(1):37-52, August 1987.

[108] Chong Wu, Juntong Qi, and Jianda Han. AESMF based sensor fault diagnosis for RUAVs. In Control and Decision Conference (CCDC), 2012 24th Chinese, pages 3384-3389. IEEE, 2012.

[109] Peter Young. Parameter estimation for continuous-time models-a survey. Automatica, 17(1):23-39, 1981.

[110] Nadia Zanzouri and Moncef Tagina. A comparative study of hybrid system monitoring based on bond graph and petri net modelling. In Systems, Man and Cybernetics, 2002 IEEE International Conference on, volume 4, pages 6-pp. IEEE, 2002.

[111] Ke Zhang, Bin Jiang, and Wei Chen. An improved adaptive fault estimation design for polytopic LPV systems with application to helicopter models. In Asian Control Conference, 2009. ASCC 2009. 7th, pages 1108-1113. IEEE, 2009.

[112] Xiaodong Zhang, Yong Liu, Rolf Rysdyk, Chiman Kwan, and Roger Xu. An intelligent hierarchical approach to actuator fault diagnosis and accommodation. In Aerospace Conference, 2006 IEEE, pages 15-pp. IEEE, 2006.

[113] Feng Zhao, Xenofon Koutsoukos, Horst Haussecker, Jim Reich, and Patrick Cheung. Monitoring and fault diagnosis of hybrid systems. Systems, Man, and Cybernetics, Part B: Cybernetics, IEEE Transactions on, 35(6):1225-1240, 2005. 


\section{Apéndice $\mathbf{A}$}

\section{Unmanned Aerial Systems Reliability Survey (July 29-2009)}

The UAS development for non-military activities is notable. A civil market with a growing number of potential applications is emerging, and the promotion of research is playing a significant role to achieve the desired integration of UAS within civil scenarios. During the last years, UVS International has been contributing with outstanding efforts in order to introduce a necessary regulatory framework allowing full and seamless integration of UAS within civilian activities.

According to the efforts of UVS international, the Technical University of Madrid -UPM is concerned about the frequency and incidence of accidents caused by UAS failure while performing any task within civilian environments. In addition, few attempts to solve this problem have been made in Europe for identifying the causes and including the required regulation to define and standardize the foundations and routines for the use of UAS in order to decrease the percentages of failure. Consequently, UPM is convinced that a community approach to this particular issue, could provide, the appropriate efforts on participating with a feasibility study that involve the UAS international community within this process.

Considering that UAS will probably share the airspace with manned airships in a near future, it becomes essential to collect information -from the UAS community- related to incidents of aircraft failure, experiments performed, and possible causes. This information will provide the foundations for developing requirements when evaluating a regulation in the field. 


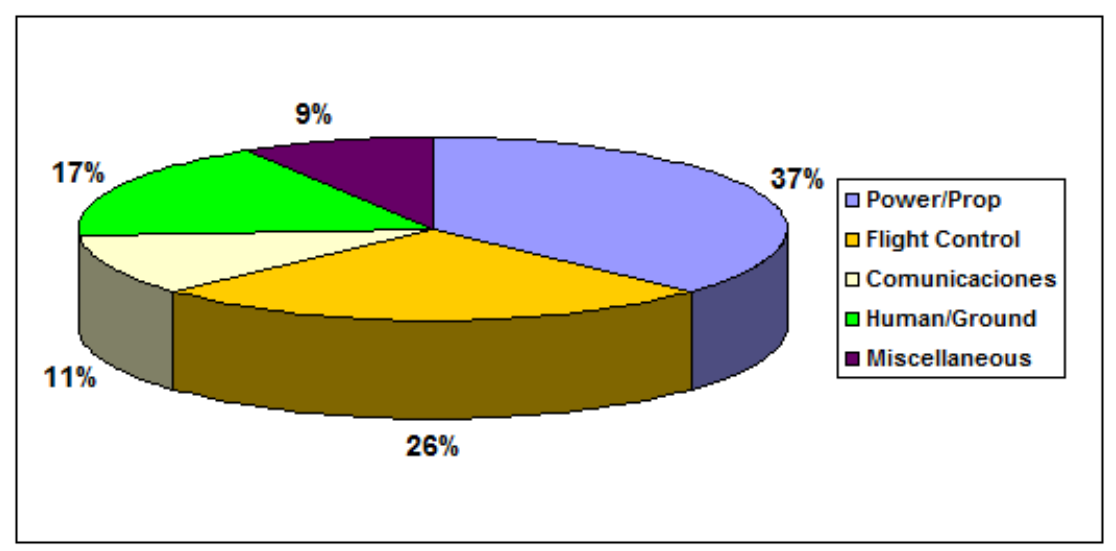

Figura A.1: UAV failure average during flights in the U.S

Herewith we would like to highlight the initiative of UVS International, and unite efforts and guidelines to carry out this survey.

\section{Antecedents}

Accidents involving UAS can be classified depending on the accident: material damage, or civilian harm. In addition causes of failure are also classified as a function of deficiencies in: Power/Propulsion, Flight Control, Communication, Ground Control / Human Factors, Miscellaneous (Other). According to data provisioned by the Department of Defense The United States of America, causes of failure are distributed in the following chart:

\section{Purpose}

The purpose of this survey is to examine the reliability of entities belonging to UVS International, in order to allow assessment of the potential risk posed by their use within civilian environments, and consequently to develop airspaceregulations to improve on: reliability, availability and effectiveness of the UAS missions.

From these criteria is important to emphasize on the affordability (acquisition), the availability of the mission (logistics and operational issues), and the acceptance within the civilian airspace (regulatory issues). These improvements offer a potential savings by the reduction in maintenance per flying hours and 
APÉNDICE A. UNMANNED AERIAL SYSTEMS RELIABILITY SURVEY (JULY 29-2009)

decreasing supply of spares, as well as aircraft attrition.

As main result of this survey, the following issues are the most valuable in order to formulate regulations.

- What kind of UAS are the most susceptible to failure/accident?

- Which are the possible causes of UAS failure?

- How often a failure occurs during flight?

- How serious (damage caused) are the accidents as consequence of UAS failure?

- How much is the importance of the human factor as cause of UAS failure?

The results will be useful for all community related UVS (manufacturers, users, government), taking into account the interest of regulatory entities (high authorities) in the management of Light UAS.

\section{Work Methodology.}

We intend to carry out this study of reliability in contribution with UVS International. For instance, we will carry out a pilot study in to level of our community, for subsequently present the final survey of the study. Once the formats of the survey are the appropriate, they will be distributed through UVS International with the purpose of reaching to the whole members of the association. Once results have been analyzed, they will be useful for all the community related to UVS (manufacturers, users, administration), which are the first interested about knowing which are the mission requirements and procedures in order to avoid UAV damage.

\section{References}

[1]. Unmanned Aerial Vehicle Reliability Study, Office of the Secretary of Defense, United States of America, February 2003.

[2]. Earth Observations and the Role of UAVs:A Capabilities Assessment, Cheryl Yuhas, NASA Science Mission Directorate, August 2006.

[3]. Army Accident Investigations and Reporting, Headquarters Department of the Army Washington, DC. 6 March 2009

[4]. A Summary of Unmanned Aircraft Accident/Incident Data: Human Factors 
APÉNDICE A. UNMANNED AERIAL SYSTEMS RELIABILITY SURVEY (JULY 29-2009)

Implications, Kevin W. Williams, Civil Aerospace Medical Institute Federal Aviation Administration Oklahoma City, December 2004.

[5]. Documentation of Sensory Information in the Operation of Unmanned Aircraft Systems, Kevin W. Williams, Civil Aerospace Medical Institute, Oklahoma City, October 2008

\section{Instructions to fill the survey}

- Please complete one form for each UAS failure/accident to supply.

- The form(s) should be emailed to: antonio.barrientos@upm.es

- The abbreviation UAS (Unmanned Aerial System) refers to: The aircraft (UAV), the UAV onboard control system, and the ground control system.

The form is composed by 20 -questions about:

Related to the aircraft:

1. Aircraft type: the UAV type (fixed wing, helicopter, quadrotor, etc) must be specified.

2. UAS characteristics: to supply information about the UAS manufacturer, including information whether the system is a commercial prototype, or own development (please include commercial subsystems -if apply-).

3. Does some type of hardware redundancy compose the Flight Control System? Please specify all the redundant subsystems, instruments (e.g. Inertial Measurement Unit - IMU, extra power supplies, etc).

4. Does the control system integrate an auto-diagnosis system? Please indicate the auto-diagnosis system capabilities -if apply- (e.g. low-battery alarm, GPSdenied environment detection).

5 . Is there some kind of reactive control to handle lack of communications? Please specify how this control works when a communication failure is detected.

Related to the mission:

6. Mission date.

7. Number of missions between failures: to specify the number of performed 
APÉNDICE A. UNMANNED AERIAL SYSTEMS RELIABILITY SURVEY (JULY 29-2009)

missions between the current accident and a previous one. For a first accident case, specify the number of missions since the UAV is on flight.

8. Mission time average: refers to the time measured since the first take-off to final landing.

9. Mission risk: refers to the quantification of the degree of risk associated to a UAV mission performance (e.g. flight in narrow scenarios with obstacles).

Related to the failure:

10. Possible cause of the accident: indicates which system/subsystem(s) malfunctioning (percentage of influence over the UAV failure).

11. Operation mode: UAV mode: manual, automatic, etc. (when the failure occurred).

12. Mission phase: take-off, hovering, cruise flight, landing, etc. (when the failure occurred).

13. Damage cost of the accident (detailed as possible).

14. Human risk/harm within the mission.

Related to the human factor:

15. Human errors: detailed description of human errors during mission.

16. Display information feedback: which kind of information provides the system to the ground control station operator.

17. Experience of the pilot (skills and flight hours) in relation to the UAV in manner.

18. Is there an independent operator to control de Aircraft and the payload?

19. Preventive measures after the accident (e.g. analysis of causes, etc).

\section{Survey}

Please complete one survey for each accident to be reported

1. Aircraft type

a. Fixed wing -Plane 
b. Rotary-wing-Helicopter

c. Rotary-wing-Quadrotor

d. Other. Which one?

2. Describe UAS characteristics:
a. Weight
b. Length
c. Span (Wingspan)
d. Maximum height of flight
e. Maximum flight distance
f. Maximum flight time (endurance)
g. Maximum Take Off Weight (Payload)
h. UAS manufacturer (commercial or prototype)
i. Cost of the system, excluding the payload:
- Less than $€ 50,000$
- Between $€ 50,000$ and $€ 150,000$
- More than $150,000 €$

3. FCS or other UAS sub-system include redundancy?
a. NO
b. YES

- Specify

4. FCS self diagnosis
a. NO
b. YES

- Specify

5. Automatic reaction after communication loss
a. Return to homepoint.
b. Go to safe position
c. Continuing with the mission
d. Other _- which?

6. Mission Date
a. 2009 Year
b. before 2009 year

7. Number of missions between failure
a. one mission
b. between 1 and 5 
c. between 5 and 10

d. more that 10

8. Average time of the mission (in hours)?

9. Mission Risk
a. Low
b. Medium
c. High

10. Possible cause of accident
a. Power / Propulsion $\%$
b. Flight Controls $\%$
c. Communications $\%$
d. Human errors $\%$
e. Miscellaneous $\%$

11. Operation mode of the aircraft when the incident happen
a. Manual
b. Automatic
c. Semi-automatic
d. Emergency

12. Mission phase when the incident happen
a. On land
b. Taking-off
c. Cruise Flight
d. Landing

13. Classify the costs of damage:

a. Low-cost (could be repaired immediately)

b. High-cost (suspension of the mission)

c. Non-reparable (total loss of the aircraft)

14. Potential human risk:
a. Low-risk (human harmless)
b. Medium-cost
c. High-risk

15. If the accident is due to human errors, the causes are:

a. Non-alerts / alarms on the interface (display)

b. Weakness in the design of the interface 
c. Procedural errors

d. Errors based on training

e. Other, which?

16. Identify the variables displayed in the interface of the ground control station:

a. Indication of active communication

b. RPM of motors

c. Battery Voltage

d. Oil Temperature (Engine)

e. Engine temperature

f. Vibrations

g. Air velocity

h. Level of fuel (petrol or battery)

i. Attitude

j. Aircraft speed

k. Position

I. Artificial horizon

m. Compass

n. Other which?

17. Indicate the experience of the pilot in UAS operation (flight hours):

a. Less than 100 hours

b. Between 100 and 300 hours

c. Over 300 hours

18. Indicate the experience of the pilot in the operation of the crashed UAS (flight hours):
a. Less than 25 hours
b. Between 25 and 100 hours
c. Over 100 hours

19. Is there an independent operator to control the aircraft and the payload?
a. Yes
b. No

20. Preventive measures after the accident?

a. Development or modification of procedures and routines.

b. $\mathrm{H} / \mathrm{W}$ redundancy.

c. $S / W$ redundancy.

d. $H / W$ modifications (includes both mechanical and electronics). 
APÉNDICE A. UNMANNED AERIAL SYSTEMS RELIABILITY SURVEY (JULY 29-2009)

e. S/W modifications.

f. Incorporation of auto-diagnosis system.

g. None 